\title{
Dynamic regulation of myelin genes in wild type and SHARP1 and -2 double null-mutant mice
}

\section{Dissertation}

\author{
for the award of the degree \\ ,Doctor rerum naturalium' \\ of the Georg-August-Universität Göttingen
}

\author{
submitted by \\ Lisa Reinecke \\ from Höxter, Germany
}

Göttingen, 28.03.2012 
Prof. Klaus-Armin Nave Ph.D. (Reviewer)

Department of Neurogenetics, Max-Planck-Institute of Experimental Medicine, Göttingen

Prof. Dr. André Fischer (Reviewer)

Laboratory for Aging and Cognitive Diseases, European Neuroscience Institute, Göttingen

Prof. Dr. Dr. Hannelore Ehrenreich

Department of Clinical Neurosciences, Max-Planck-Institute of Experimental Medicine, Göttingen

Declaration

Herewith I declare that I prepared the PhD thesis entitled ,Dynamic regulation of myelin genes in wild type and SHARP1 and -2 double null-mutant mice' on my own and with no other sources and aids than quoted. 


\section{ACKNOWLEDGEMENTS}

An erster Stelle möchte ich Moritz für ein vielseitiges und herausforderndes Projekt danken, bei dem ich sehr viel lernen konnte. Ich danke ihm für seinen aufbauenden Optimismus, Diskussionen und sein Verständnis für meine Familie.

Bei Klaus möchte ich mich für die herausragende Möglichkeit bedanken, die Arbeit in einem so gut ausgerüsteten Labor mit einer Menge know how durchführen zu können.

Ich danke Klaus sowie Prof. Fischer und Prof. Ehrenreich als Mitglieder meines Thesis Komitees für die Zeit, die sie sich für mein Projekt genommen haben sowie für die konstruktiven Ratschläge, die ich von ihnen erhalten habe. Ich möchte meinen Dank auch auf die Mitglieder meiner Prüfungskommission, Prof. Simons, Prof. Oster und Prof. Hörner, ausweiten.

Bei Prof. Hörner möchte ich mich ganz herzlich für die Gespräche und Ratschläge bedanken, die mit dazu geführt haben, diese Arbeit fertig zu stellen.

Ein ganz großer und sehr herzlicher Dank gilt Magda und Micha, die mir bei Fragen und Problemen jederzeit geduldig zur Seite standen und gerade während des Schreibens der Arbeit eine enorme Unterstützung waren.

Bei Sven möchte ich mich ganz herzlich für sämtliche Hilfe und Geduld in technischen Notfällen bedanken $;$ und natürlich für den Beistand beim Formatieren.

Micha, Magda, Ali und Anna danke ich für das Korrekturlesen der Arbeit.

Des weiteren gilt ein großer Dank der gesamten Rossner Arbeitsgruppe, für ihre ständige Hilfsbereitschaft, den Zusammenhalt und die tolle Arbeitsatmosphäre. Und ganz besonders für die Hilfe während der Schwangerschaft(en) $)$. Euch werde ich alle vermissen!!!

Vielen Dank, Ali, für deine Hilfsbereitschaft in allen Dingen und für die Diskussionen über unsere Projekte. Und ganz vielen Dank, Katrin, für die enorme Unterstützung bei den VirusExperimenten.

Ich möchte Julia Patzig und Patricia Monasterio für die Hilfe beim Mikroskopieren danken, sowie Sebastian Schmitt (AG Simons) für seine Unterstützung bei der Transfektion der primären Oligodendrozyten.

Und vielen Dank an Aiman, der sich immer Zeit für hilfreiche Diskussionen genommen hat.

Ulli und Annette möchte ich nicht vergessen zu erwähnen, die stets in der Zellkultur und in der Histologie zur Seite standen sowie Gabriele und Michaela für die organisatorische Hilfe.

Last but not least gilt der allergrößte Dank meiner Familie, ohne deren ausnahmslose Unterstützung diese Arbeit nicht möglich gewesen wäre. Nennen möchte ich vor allem meine Mutter und Johannes, die mir gerade während des Schreibens den Rücken frei gehalten haben und sich liebevoll um Julius gekümmert haben. Ein ganz großer Dank geht in der Hinsicht auch an Christiane, Johannes Eltern sowie Henriette und Hauke. 
FÜR MEINEN VATER 


\section{TABLE OF CONTENT}

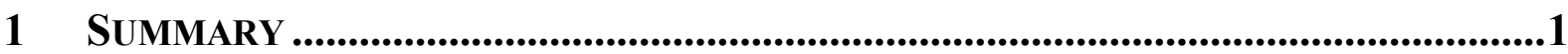

2 INTRODUCTION...........................................................................................2

2.1 The circadian system ................................................................................................. 2

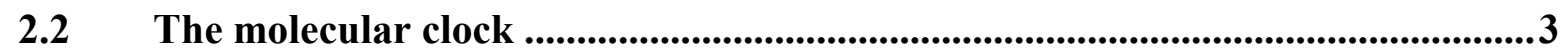

2.3 SHARP1 and -2 transcription factors........................................................................5

2.4 SHARP1 and -2 functions in the molecular clock ...........................................5

2.5 Altered clock gene expression in SHARP1 and -2 single and double nullmutant mice................................................................................................................

2.6 Altered sleep/wake behaviour in SHARP1 and -2 double null-mutant mice......8

2.7 Attenuated activity-dependent gene expression in SHARP1 and -2 double null-mutant mice.....................................................................................................................11

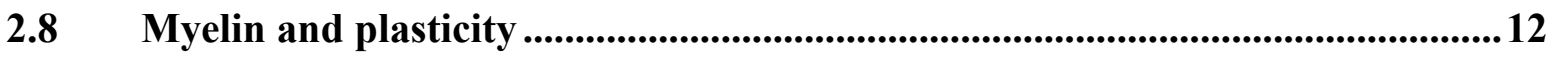

2.9 Neurotransmitters implicated in myelination ...............................................14

2.10 Myelin and psychiatric disorders .....................................................................14

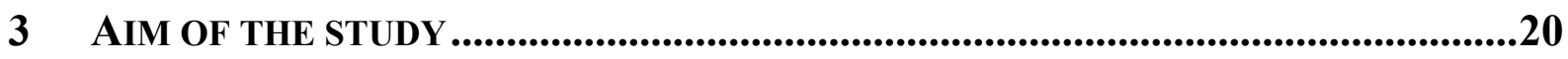

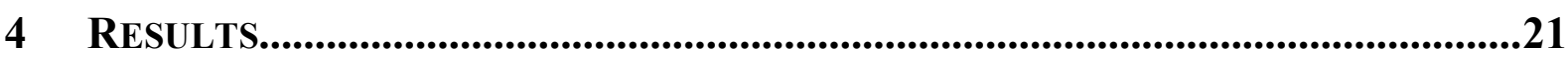

4.1 Behavioural analyses ............................................................................................21

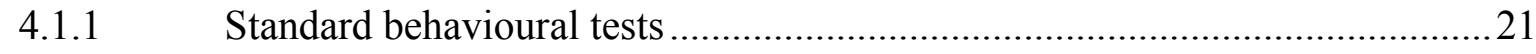

4.1.2 Increased activity and reduced anxiety levels in $\mathrm{S} 1 / 2^{-/-}$mice in the open

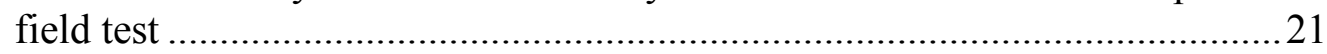

4.1.3 Decreased curiosity of $\mathrm{S} 1 / 2^{-/-}$mice in the hole board test ..............................22

4.1.4 No differences in sensory processing in $\mathrm{S} 1 / 2^{-/-}$mice in the hot plate test.......23

4.1.5 Tendency towards increased motivational behaviour of $\mathrm{S} 1 / 2^{-/-}$mice in the tail suspension test ..............................................................................2 24

4.2 Dynamic regulation of myelin genes in precise grey and white matter micro-areas of the brain ...................................................................................25

4.2.1 Circadian and activity-dependent regulation of myelin genes in the ACC micro-area in WT mice and attenuated effects in $\mathrm{S} 1 / 2^{-/-}$mice .......................28

4.2.2 Circadian and activity-dependent regulation of myelin genes in the SSMCX micro-area in WT mice and attenuated effects in $\mathrm{S} 1 / 2^{-/-}$mice ..........32

4.2.3 Circadian and activity-dependent regulation of myelin genes in the $\mathrm{CC}$ micro-area in WT mice and attenuated effects in $\mathrm{S} 1 / 2^{-/-}$mice ........................35

4.2.4 Activity-dependent regulation of myelin genes in the CC micro-area in WT mice 
4.3 Attenuated regulation of myelin genes in the sciatic nerve and optic nerve in $W T$ and $S 1 / 2^{-/-}$mice .................................................................................................. 40

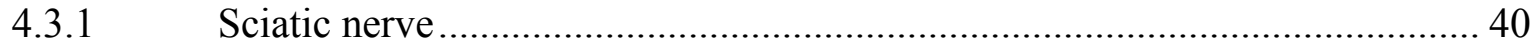

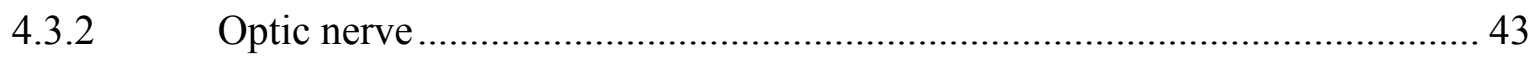

4.4 Circadian stability of myelin protein levels in the prefrontal cortex and corpus callosum in WT and $S 1 / 2^{-/-}$mice ............................................................. 47

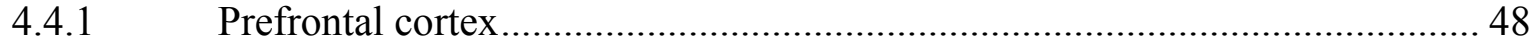

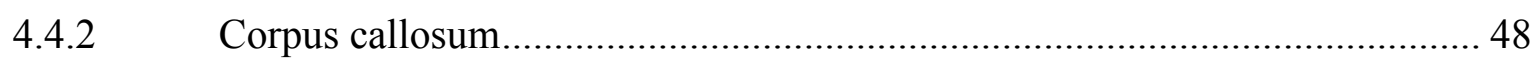

4.5 Differential regulation of myelin gene expression upon sleep deprivation in

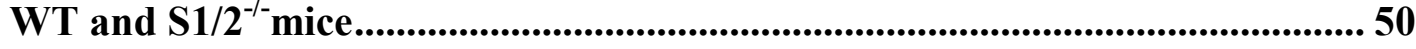

4.6 Dynamic expression of myelin genes in stimulated primary oligodendrocyte cultures and reduced expression levels in $\mathrm{S} 1 / 2^{-/-}$cultures .................................. 53

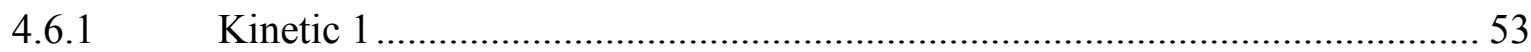

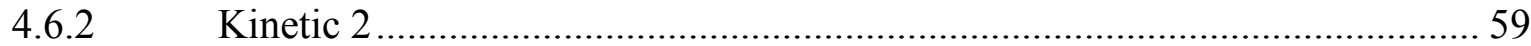

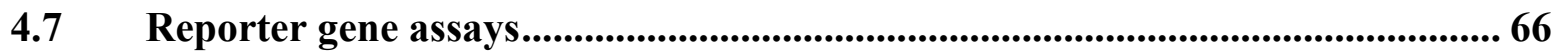

4.7.1 Repressive and co-activating functions of SHARP1 and -2 functions in a

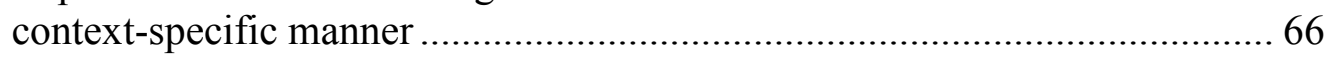

4.7.2 Effects of SHARP proteins on myelin promoter activity in vitro ................... 69

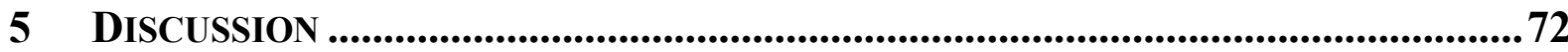

5.1 Behavioural alterations reminiscent of psychiatric diseases in $\mathrm{S} 1 / 2^{-/-}$mice...... 72

5.2 Dynamic regulation of myelin genes in WT mice .......................................... 73

5.2.1 Dynamic day time-dependent regulation .................................................. 75

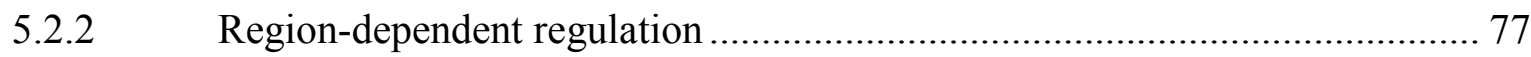

5.3 Attenuated expression of myelin genes in $\mathrm{S} 1 / 2^{-/-}$mice ....................................... 79

5.3.1 Regulation of myelin genes in response to sleep deprivation......................... 80

5.3.2 Consequences with respect to the metabolism............................................ 81

5.3.3 Consequences with respect to psychiatric disorders ..................................... 82

5.3.4 Reduced expression levels of myelin genes in primary cultured oligodendrocytes from $\mathrm{S} 1 / 2^{-/-}$mice....................................................... 82

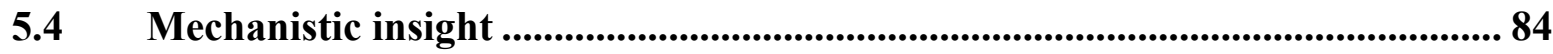

$5.5 \quad$ Concluding remarks ................................................................................................. 86

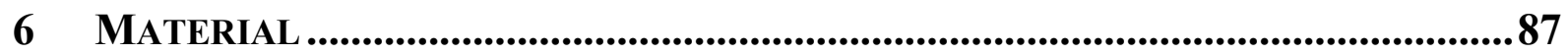

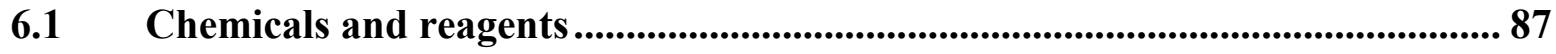

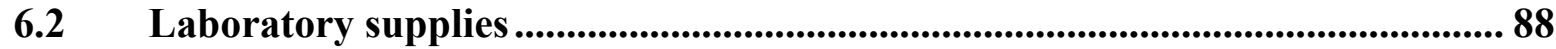

6.3 Laboratory equipment....................................................................................... 88

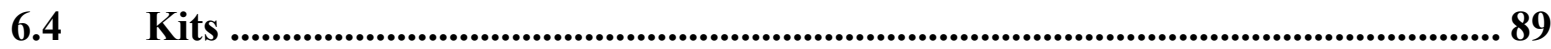




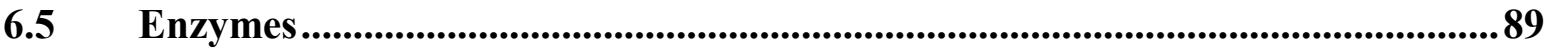

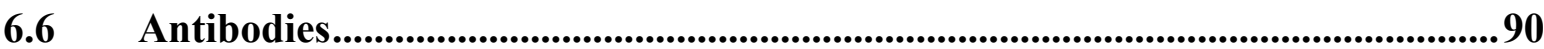

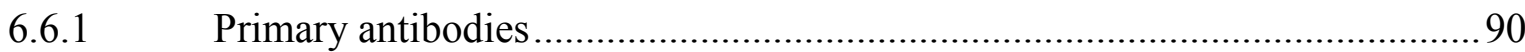

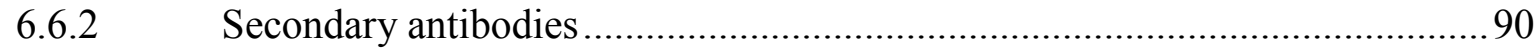

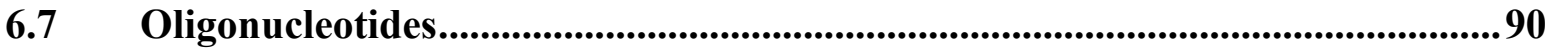

6.7.1 Oligonucleotides for genotyping of SHARP1 and -2 null mutant mice..........90

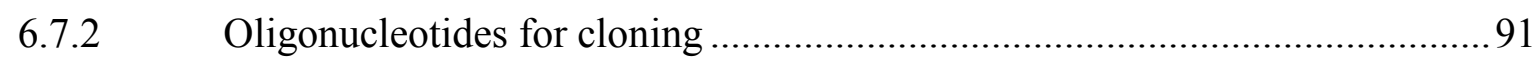

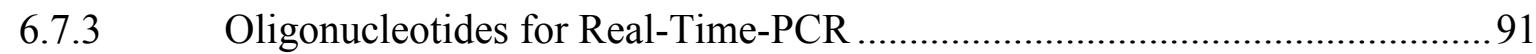

Oligonucleotides for cDNA synthesis ....................................................... 92

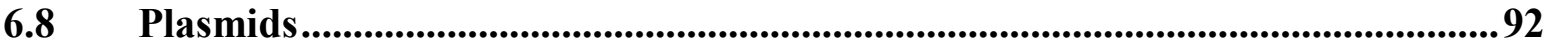

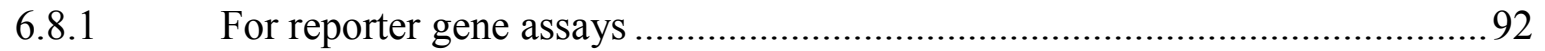

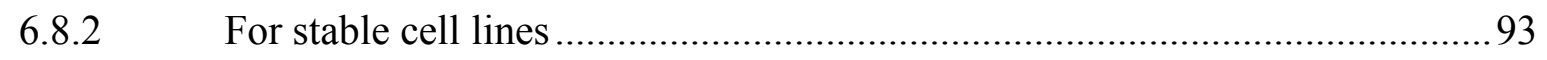

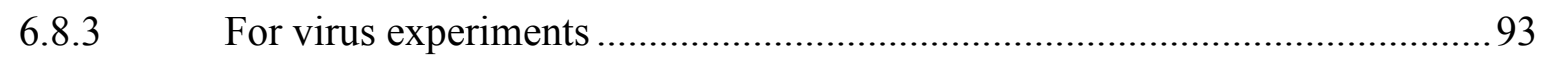

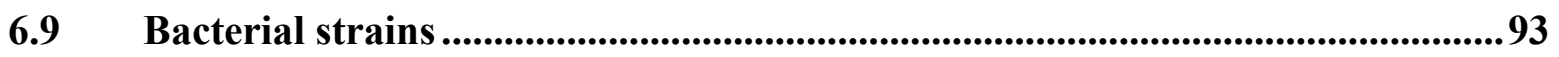

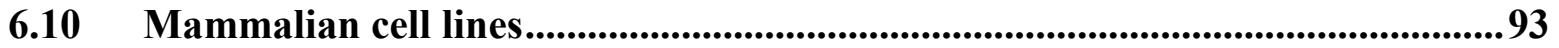

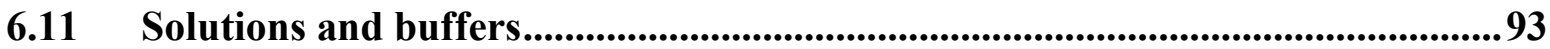

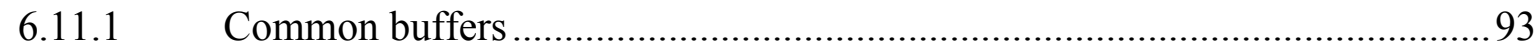

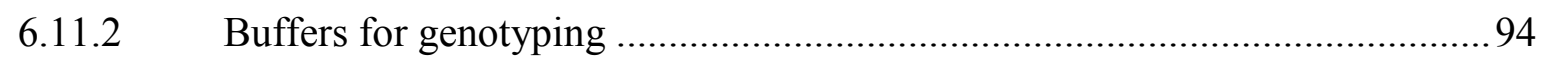

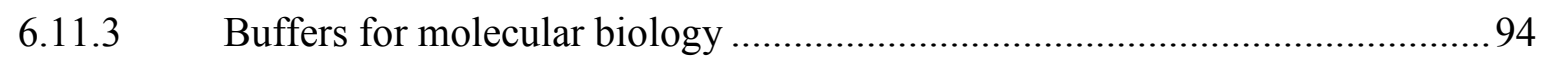

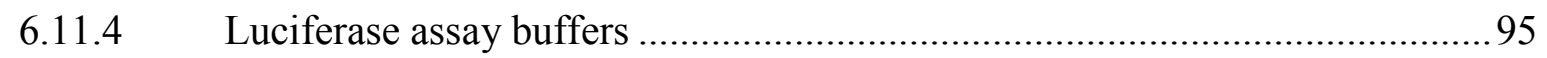

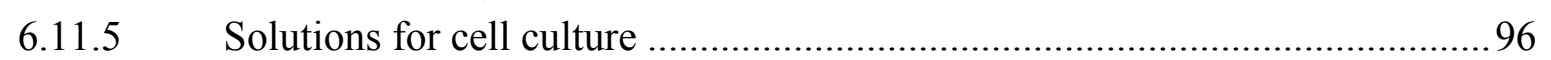

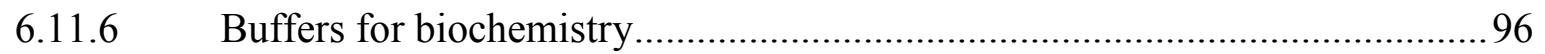

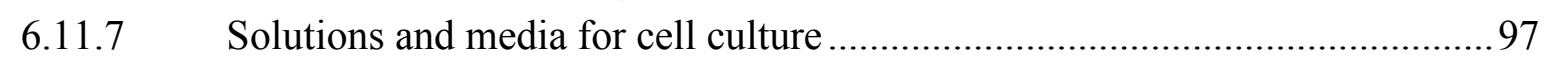

6.11.8 Buffers for immunohistochemistry and immunocytochemistry .....................99

7 METHODS...........................................................................................................101

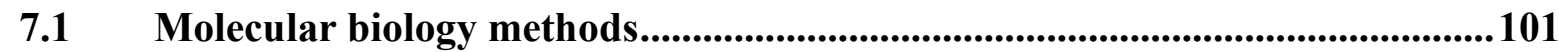

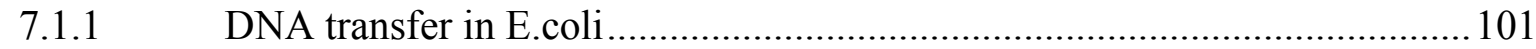

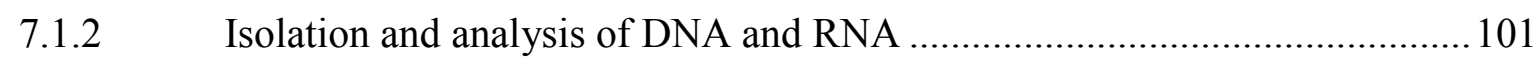

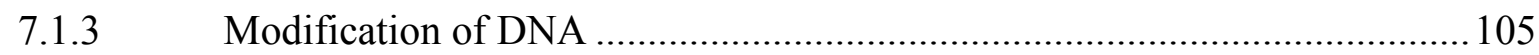

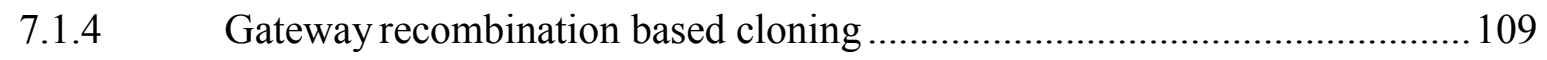

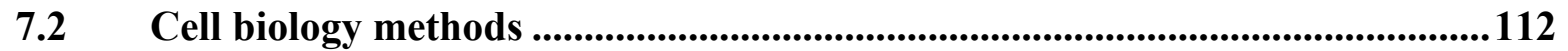

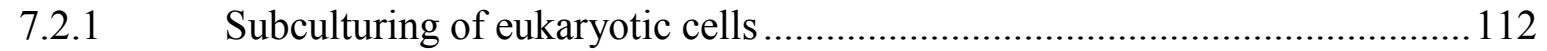

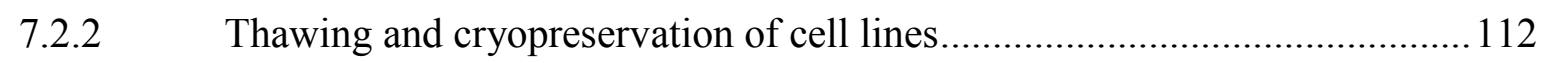

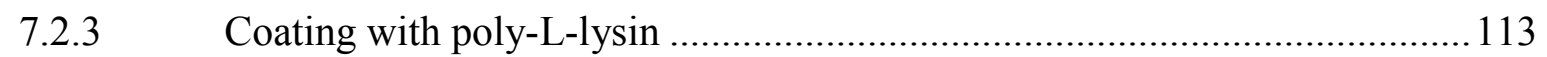

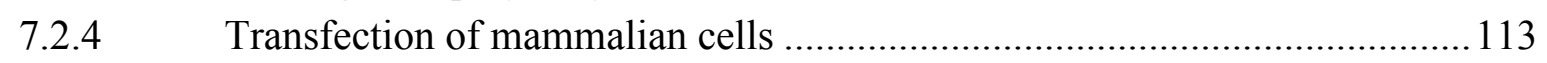

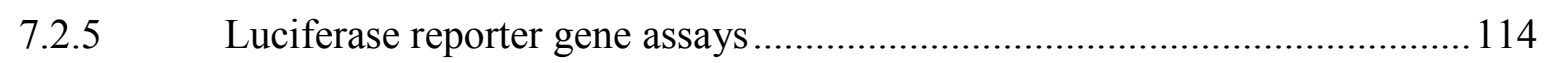

7.2.6 Preparation of primary mouse oligodendrocytes........................................ 115

7.2.7 Preparation of primary mouse neurons.................................................... 115 
7.3 Generation of stably transfected Oli-neu cell lines ......................................... 116

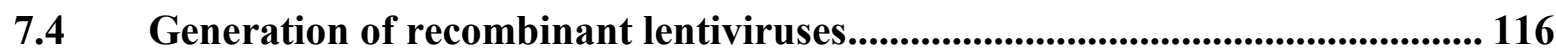

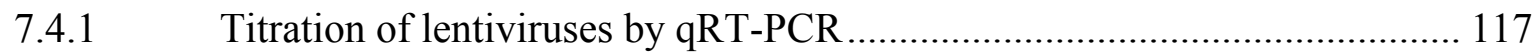

7.4.2 Infection of stably transfected Oli-neu cell lines ....................................... 118

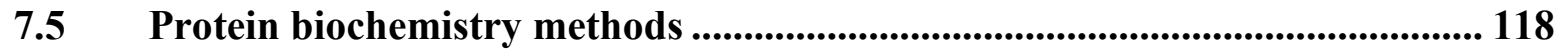

7.5.1 Preparation of protein extracts from mouse tissue....................................... 118

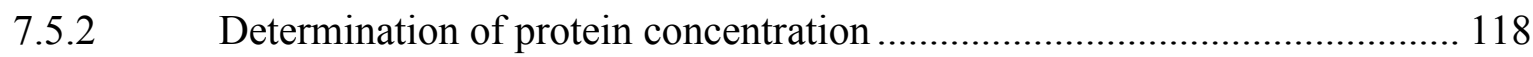

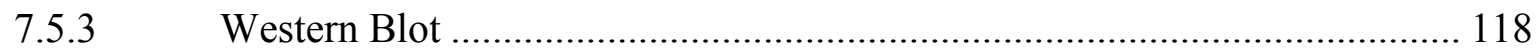

7.6 Histological methods ....................................................................................... 120

7.6.1 Preparation of paraffin embedded sections ................................................. 120

7.6.2 DAB staining of paraffin embedded tissue ............................................... 120

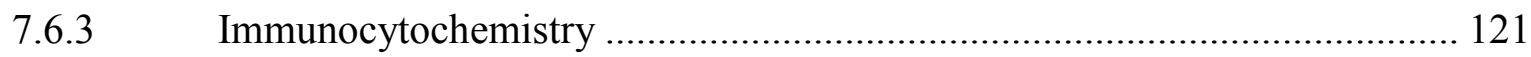

7.6.4 Preparation and dehydration of cryosections for LCM.............................. 121

Laser-captured microdissection................................................................................... 122

7.8 Production and maintenance and transgenic mice ......................................... 122

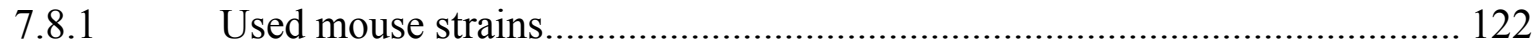

7.8.2 Housing conditions of experimental animals.............................................. 123

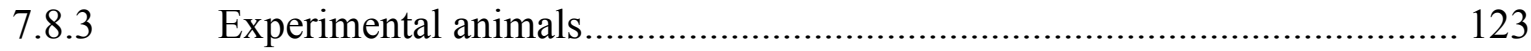

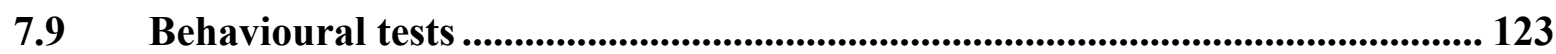

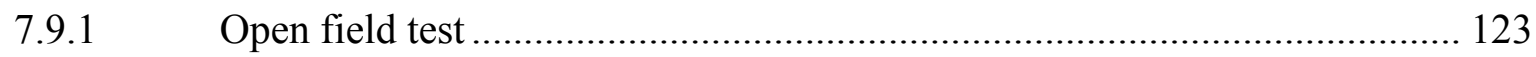

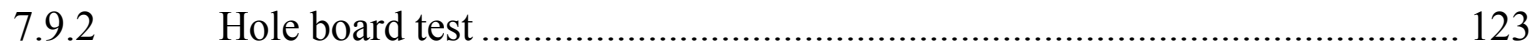

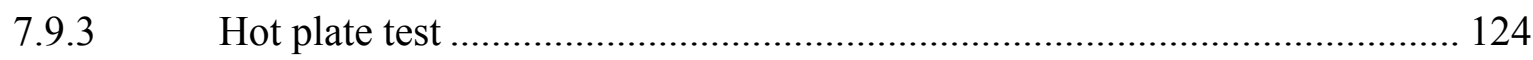

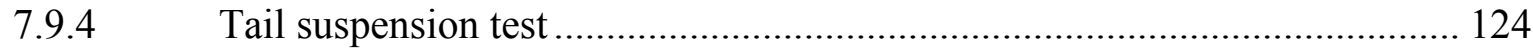

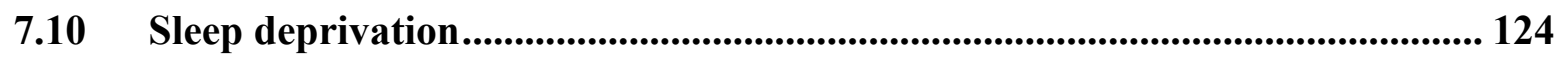

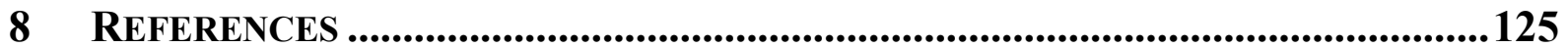

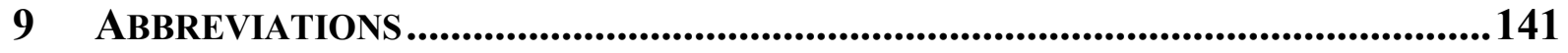

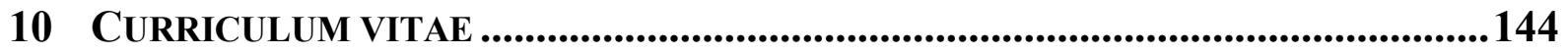




\section{LIST OF FIGURES}

Fig. 1 Simplified model of the circadian molecular clock in the SCN ................................ 4

Fig. 2 SHARP1 and -2 functions in the molecular clock................................................. 6

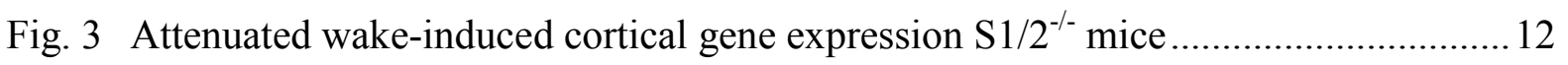

Fig. 4 Increased activity and reduced anxiety levels in $\mathrm{S} 1 / 2^{-/-}$mice in the open field

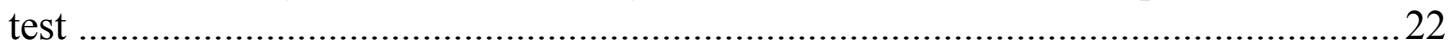

Fig. 5 Decreased curiosity of $\mathrm{S} 1 / 2^{-/-}$mice in the hole board test .....................................2 23

Fig. 6 No differences in sensory processing in $\mathrm{S} 1 / 2^{-/-}$mice in the hot plate test and a tendency towards increased motivational behaviour in the tail suspension test..........25

Fig. 7 Schematic representation of the brain areas and nerves taken for analysis ................26

Fig. 8 Experimental design: Housing conditions and analysed time points .........................27

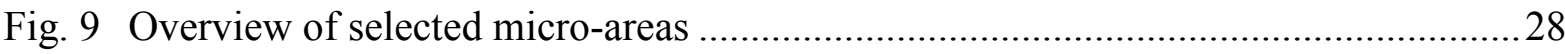

Fig. 10 Circadian and activity-dependent regulation of myelin genes in the ACC microarea in WT mice and attenuated effects in $\mathrm{S} 1 / 2^{-/-}$mice.......................................... 31

Fig. 11 Circadian and activity-dependent regulation of myelin genes in the SSMCX micro-area in WT mice and attenuated effects in $\mathrm{S} 1 / 2^{-/-}$mice ...................................34

Fig. 12 Circadian and activity-dependent regulation of myelin genes in the $\mathrm{CC}$ micro-

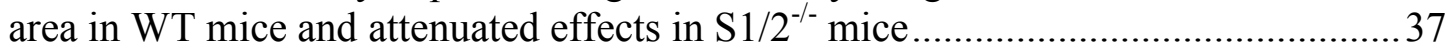

Fig. 13 Circadian and activity-dependent regulation of myelin genes in the $\mathrm{CC}$ microarea in WT mice

Fig. 14 Attenuated regulation of myelin genes in the sciatic nerve in WT and S1/2 ${ }^{-/-}$ mice

Fig. 15 Attenuated regulation of myelin genes in the optic nerve in $\mathrm{WT}$ and $\mathrm{S} 1 / 2^{-/-}$mice under LD and DD conditions

Fig. 16 Circadian stability of myelin protein levels in the prefrontal cortex and corpus callosum in WT and $\mathrm{S} 1 / 2^{-/-}$mice.

Fig. 17 Differential regulation of myelin gene expression upon sleep deprivation in WT and $\mathrm{S} 1 / 2^{-/-}$mice

Fig. 18 Experimental design of primary cultured oligodendrocyte stimulation with $\mathrm{NCM}$

Fig. 19 Immunocytochemical and western blot analysis of oligodendrocyte cultures .54

Fig. 20 Dynamic expression of myelin genes in stimulated primary oligodendrocyte cultures and reduced expression levels in $\mathrm{S} 1 / 2^{-/-}$cultures 1

Fig. 21 Experimental design of primary oligodendrocyte stimulation with NCM

Fig. 22 Immunocytochemical analysis of primary oligodendrocyte cultures.

Fig. 23 Dynamic expression of myelin genes in stimulated primary oligodendrocyte cultures and reduced expression levels in $\mathrm{S} 1 / 2^{-/}$cultures 2

Fig. 24 Repressive and co-activating functions of SHARP1 and -2 in a context-specific manner 
Fig. 25 SHARP2 increases myelin promoter activity in stably transfected Oli-neu cell lines

Fig. 26 SHARP2 increases myelin promoter activity in stably transfected Oli-neu cell lines 71

Fig. 27 Schematic representation of the three-fragment recombination cloning 111

Fig. 28 Schematic representation of the pEXPR Mal/Mbp prom EXT (GL4) reporter constructs 


\section{SUMMARY}

In living organisms, a molecular clockwork synchronises physiological mechanisms with daily recurrent changes in the environment, a process that is termed entrainment. The two basic helix-loop-helix (bHLH) transcription factors SHARP1 (DEC2) and SHARP2 (DEC1, Stra13) are important amplitude modifiers of this clockwork. Both factors play important roles in the adaptation to altered light/dark cycles and in the homeostatic control of sleep. Moreover, sleep/wake and behavioural analysis of SHARP1 and -2 double mutant $\left(\mathrm{S} 1 / 2^{-/}\right)$ mice revealed characteristic features reminiscent of psychiatric endophenotypes. Unexpectedly, microarray analysis identified genes encoding myelin proteins among sleep/wake-dependent transcripts in the cerebral cortex of wild type (WT) mice, whereas this regulation appeared to be attenuated in $\mathrm{S} 1 / 2^{-/-}$animals. Thus, a major aim of this thesis was to corroborate these findings with more precise sampling procedures, including a high spatial and temporal resolution. Laser-captured microdissection (LCM), together with quantitative RT-PCR was applied to analyse a potential circadian and/or activity-dependent pattern of myelin gene expression in precisely defined forebrain areas in WT mice as well as a deregulation in $\mathrm{S} 1 / 2^{-/}$mice. Indeed, I detected a novel plasticity of myelin genes on mRNA level which appeared to be time- and region-specific in WT animals. This finding is of particular interest as adult plasticity of white matter tracts was recently discussed in response to learning. However, these effects were attenuated in $\mathrm{S} 1 / 2^{-/-}$mice. Intriguingly, a deregulation of myelin genes is also reported for psychiatric diseases like schizophrenia, bipolar disorder and depression. S1/2/- mice also showed an attenuated response of some myelin genes upon sleep deprivation, which potentially reflects the function for SHARP proteins as homeostatic integration factors both in mice and humans. The expression of myelin genes was also reduced in stimulated primary cultured oligodendrocytes isolated from $\mathrm{S} 1 / 2^{-/-}$mice, thus reflecting the in vivo findings. Consistently, overexpressed SHARP proteins activated $\mathrm{Mal}$ and $\mathrm{Mbp}$ promoters, as shown in luciferase reporter gene assays in Oli-neu cells and primary oligodendrocytes.

In summary, myelin gene expression for the first time was analysed in precise cortical regions indicating a novel high plasticity. In S1/2 ${ }^{-/}$mice, these effects were reduced, potentially as SHARP proteins are integration factors that exert both repressive and co-activating functions in a context-specific manner. By linking alterations in the homeostatic processes to the etiology of psychiatric diseases the $\mathrm{S} 1 / 2^{-/-}$mouse model might contribute to the understanding of mental disorders. 


\section{INTRODUCTION}

\subsection{The circadian system}

Living organisms evolved molecular and behavioural mechanisms designed to anticipate and cope with the daily recurrent changes in life. This system is called the circadian system (Latin circa diem, 'about a day'). It deviates from the 24 hour solar cycle and needs to be constantly reset to maintain synchrony with the environment, a process that is called entrainment.

For mammals, the most prominent entrainment factor or zeitgeber (German, time-giver) is the light/dark cycle. Information about light is perceived by the retina where specialised melanopsin-containing retinal ganglion cells (RGCs) project via the retinohypothalamic tract (RHT) to our 'master' clock in the suprachiasmatic nucleus (SCN) of the ventral hypothalamus. The SCN consists of about 20000 neurons which act as single cell circadian oscillators (Reppert and Weaver, 2001). It is able to sustain 24 hour periodicity on its own, but can be entrained by environmental cues. Being on top of a hierarchy, the entrained master clock synchronises the so-called 'slave' clocks, which are dispersed in other brain regions (e.g. cortex) and peripheral organs (e.g. liver) (Reppert and Weaver, 2002). These local oscillators are independent, as they are able to sustain the 24 hour rhythm for a few days, but in the end depend on the input of the master clock to maintain rhythmicity (Reppert and Weaver, 2002). By controlling a variety of neuronal, behavioural and physiological processes, the central and peripheral clocks ensure optimal timing of the body with respect to external and internal demands (Masri and Sassone-Corsi, 2010). Auxiliary proteins keep the clock strictly timed by posttranslational modifications such as methylation, acetylation, phosphorylation and ubiquitination, but, nevertheless, the circadian timing system displays a high level of plasticity (Masri and Sassone-Corsi, 2010). Several studies reveal that about $10 \%$ of the transcriptome is under circadian control (Akhtar et al., 2002; Panda et al., 2002), but the cycling transcripts appear to be highly tissue-specific, pointing to the importance of local transcription factors influencing the clock machinery (Akhtar et al., 2002; Panda et al., 2002; Ueda et al., 2005).

The circadian clock exerts an enormous influence on mechanisms concerning our whole body. It has been known for a long time that many metabolic processes such as glucose and cholesterol metabolism are controlled by the circadian system (Demierre et al., 2005; Rutter et al., 2002), but only recently dysfunctions of core clock genes have been linked to metabolic 
diseases. For instance, homozygous Clock mutant mice which become arrhythmic in constant darkness develop classical markers of a 'metabolic syndrome' such as hyperglycemia, dyslipidemia and hypoinsulinemia as well as obesity (Turek et al., 2005). Deletion of Bmall, which results in complete arrythmicity in mice (Bunger et al., 2000) abolishes circadian fluctuations in glucose and triglyceride levels (Rudic et al., 2004). Also, shift work has been associated with the development of a metabolic syndrome and cardiovascular diseases (De Bacquer et al., 2009; Karlsson et al., 2001).

Several lines of evidence suggest that the circadian rhythm also impacts on memory consolidation. Circadian phase-shifting (experimental jet lag, leading to a desynchronisation of internal and external clocks) following Morris water maze training impairs hippocampusmediated memory consolidation (Devan et al., 2001) and also leads to reduced performance in hippocampus-dependent passive avoidance test (Tapp and Holloway, 1981). Moreover, lesions of the SCN in rats are associated with a reduction of hippocampus-associated long term potentiation (LTP) (Stephan and Kovacevic, 1978). It is known that the hippocampus displays circadian rhythms in mitogen activated protein kinase (MAPK) and cyclic adenosine monophosphate (cAMP) signalling pathways (Eckel-Mahan et al., 2008; Gerstner et al., 2009) known to be involved in memory consolidation processes (Impey et al., 1999; Sweatt, 2004; Wang and Storm, 2003).

Moreover, the circadian system has been implicated in the pathogenesis of bipolar I disorder (BPI), as patients suffering from BPI display abnormal circadian regulation regarding the sleep/wake cycle, appetite and hormonal rhythms (Lenox et al., 2002; Mansour et al., 2005). Disruption in circadian function may precede the beginning of manic episodes (Salvatore et al., 2008) and normalisation of circadian rhythms clinically stabilizes the symptoms (Mansour et al., 2009). Consistent with this, lithium, a mood stabilizer, may exert its therapeutic effects potentially by lengthening the circadian period (Abe et al., 2000; Campbell et al., 1989). Also, bright light therapy and total sleep deprivation are clinically used techniques to treat seasonal affective disorders and depression, respectively (Smeraldi et al., 1999; Terman et al., 2001).

\subsection{The molecular clock}

The molecular clockwork is composed of interacting transcriptional and posttranscriptional feedback loops which generate circadian cycles (Fig. 1). 


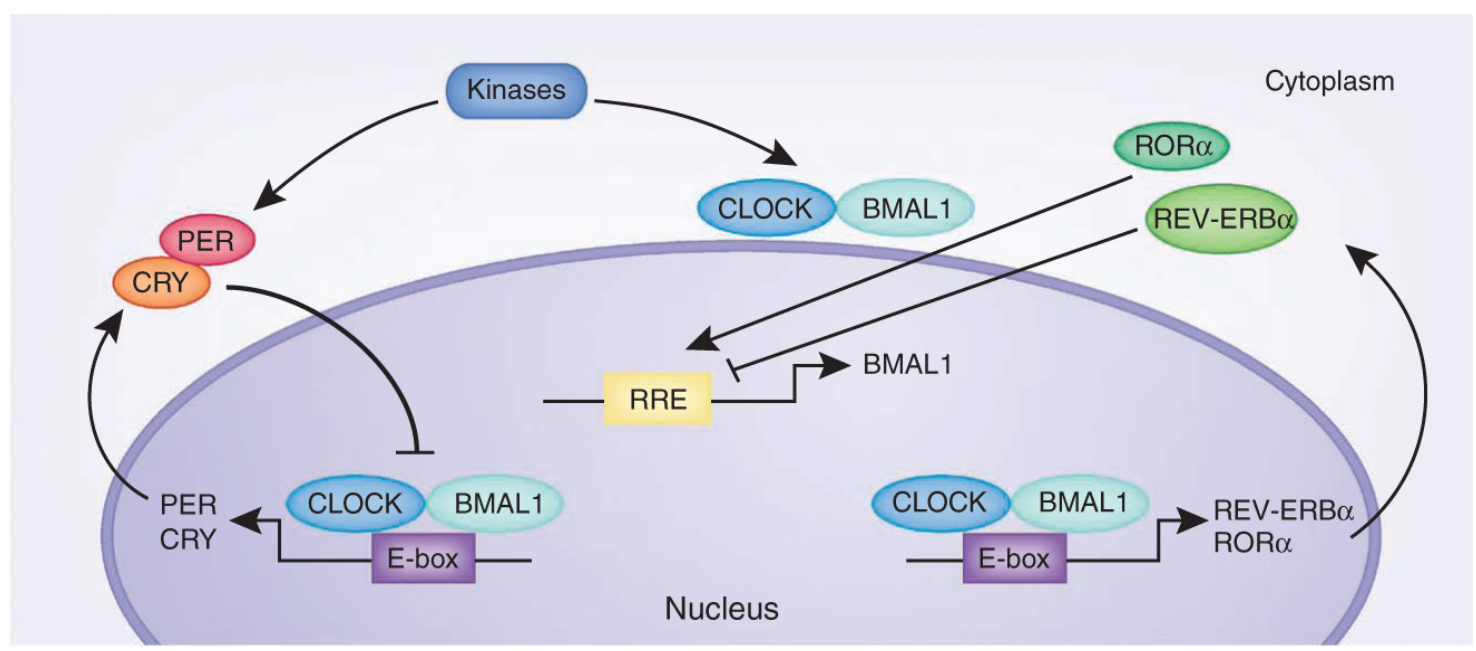

Fig. 1 Simplified model of the circadian molecular clock in the SCN

The core clock transcription factors, CLOCK and BMAL1, activate transcription of clock controlled genes (CCGs) via E-box binding. PER and CRY proteins dimerise in the cytoplasm, translocate into the nucleus and inhibit CLOCK/BMAL1 activity. CLOCK and BMAL1 also enhance Rev-Erba and Rora transcription, which in turn influence the expression of BMAL1. Additional stability of the molecular clockwork is obtained by posttranslational modifications (e.g. phosphorylation by kinases). CLOCK, circadian locomotor output cycle kaput; BMAL, brain and muscle aryl hydrocarbon receptor nuclear translocator-like factor 1; PER, period; CRY, cryptochrome; RRE, REV-ERB/ROR response element. The figure is taken from Masri and Sassone-Corsi (Masri and Sassone-Corsi, 2010).

In the $\mathrm{SCN}$, the core of the positive feedback loop is constituted by circadian locomotor output cycle kaput (CLOCK) and brain and muscle aryl hydrocarbon receptor nuclear translocator-like factor 1 (BMAL1), both basic helix loop helix (bHLH)-ARNT-SIM (PAS) transcription factors which heterodimerise via the PAS region and activate E-box mediated transcription of clock controlled genes (CCG). These CCGs include period (PER1, 2, 3) and cryptochrome $(\mathrm{CRY} 1,2)$ protein families, forming the predominatly negative feedback loop. PER and CRY proteins form complexes in the cytoplasm and translocate back to the nucleus (Reppert and Weaver, 2001). There, CRY proteins directly bind to CLOCK and/or BMAL1, thereby inhibiting transcription. PER2 ensures rhythmic expression of Bmall. High abundance of BMAL1 promotes the formation of CLOCK/BMAL1 heterodimers, which indicates the start of a new cycle (Reppert and Weaver, 2001). In the SCN at circadian time 0 (CT0, the beginning of the circadian day without entrainment by zeitgebers) CLOCK/BMAL1 heterodimers are highly abundant, activating the expression of Per and Cry. In the middle of the circadian day at CT12, PER and CRY proteins accumulate in the nucleus, where CRY inhibits CLOCK/BMAL1 mediated transcription. PER2 enhances Bmall expression, resulting in a peak of Bmall mRNA from CT15-18, followed by high abundance of BMAL1 protein with four-six hours delay. The BMAL1 protein concentration is supposed to be rate-limiting for the complex formation with CLOCK, a step that starts a new circadian cycle (Reppert and 
Weaver, 2001). Additional feedback loops comprising the orphan nuclear receptors ROR $\alpha$ and REV-ERB $\alpha$ are induced by CLOCK/BMAL1 complexes and influence Bmall transcription by operating as activator (ROR $\alpha$ ) (Nakajima et al., 2004; Sato et al., 2004b) or inhibitor (REV-ERB $\alpha$ ) (Preitner et al., 2002). These interlocked feedback loops potentially increase the stability and preciseness of circadian timing. Two new proteins, SHARP1 and -2, have been postulated as additional negative regulators of the mammalian clock (Hamaguchi et al., 2004; Honma et al., 2002; Li et al., 2004; Sato et al., 2004a).

\subsection{SHARP1 and -2 transcription factors}

SHARP1 (DEC2, also called BHLHB40, BHLHB2, Stra13) and SHARP2 (DEC1, BHLHB41, BHLH3) are members of the bHLH family of transcription factors. This group plays a role in proliferation, specification and differentiation of cortical progenitor cells (Ross et al., 2003) and is structurally characterised by a basic (b) domain responsible for DNA binding as well as an adjacent HLH domain, which mediates protein dimerisation. Based on structural and functional characteristics, bHLH proteins can be classified into different subfamilies (Murre et al., 1994). SHARP is the acronym for Enhancer-of-Split and HairyRelated Protein, derived from the mammalian homologues of Drosophila hairy and the enhancer-of-split-complex proteins (HES), which structurally display the closest similarity. HES proteins are predominately expressed in the subventricular zone and are considered as transcriptional repressors (Ishibashi et al., 1993). However, SHARP proteins differ from previously described bHLH proteins by their expression pattern in adult brain regions associated with plasticity and their coupling to neuronal activity (Rossner et al., 1997). Also, both SHARP proteins lack a carboxy-terminal 'WRPW domain', which is important in corepressor binding (Fujimoto et al., 2001; Shen et al., 1997). Therefore, Rossner and colleagues postulated that SHARP proteins constitute a new subfamily of bHLH proteins, mediating adaptive changes in the adult brain (Rossner et al., 1997).

\subsection{SHARP1 and -2 functions in the molecular clock}

Based on mRNA expression profiles in the SCN and forebrain, SHAPR1 and -2 were suggested to represent novel negative regulators of the molecular clock (Hamaguchi et al., 2004; Honma et al., 2002; Li et al., 2004; Sato et al., 2004a). Indirect evidence by reporter gene assays identified SHARP1 and -2 as repressors of CLOCK/BMAL1 mediated transactivation of the mouse Perl promoter. This occurs by direct protein interaction with 
BMAL1 and/or competition for E-box elements (Honma et al., 2002). Thereby, SHARP1 revealed a stronger repressive effect than PER1, PER2 and CRY1, whereas the repressive function of SHARP2 was comparable to that of CRY1 and CRY2 (Honma et al., 2002). SHARP1 and -2 display robust circadian expression in the SCN in constant darkness (DD) and light dark conditions ((LD, in which light as a zeitgeber is used), with a maximum of mRNA abundance in early (Sharp2) to middle (Sharp1) circadian day. Sharp1 expression was also detected outside the SCN in the caudate putamen, pineal gland, and granule cells in the cerebellum. Sharp2 is strongly expressed in the cerebral cortex, particularly in the fifth layer, thalamus and superior colliculus. Expression of both genes was found in the olfactory bulb, piriform cortex, hippocampus and hypothalamic nuclei (Honma et al., 2002). As Sharpl and 2 expression is enhanced by CLOCK/BMAL complexes (Honma et al., 2002), it is suggested that these proteins form an additional autoregulatory feedback loop (Fig. 2).

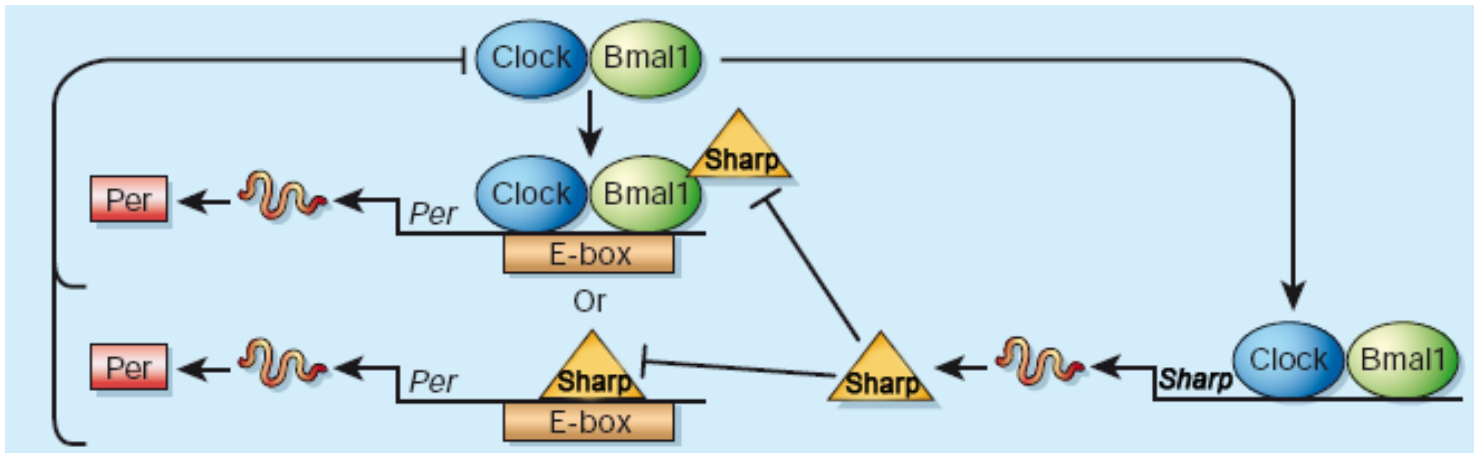

Fig. 2 SHARP1 and -2 functions in the molecular clock

BMAL1 increases Per and Sharp transcription, PER in turn positively feeds back and Bmall (Yu et al., 2002), whereas SHARP stops transactivation of Per, thereby closing the loop (Honma et al., 2002). The figure is taken and modified from Alvarez and Sehgal (Alvarez and Sehgal, 2002).

Supporting for this is that SHARP1 and -2 bind to two E-box elements in the Sharp1 promoter, and that it was shown that also CRY and PER exert repressive effects on Sharp1 expression (Hamaguchi et al., 2004). The fact that Sharp1 and -2 display circadian oscillation in the SCN and liver hints at a key role for the transcription factors in circadian regulation (Honma et al., 2002). Light-induced activation of Perl and Sharp2 in the SCN points to an important function of the SHARP proteins as entrainment factors (Honma et al., 2002). Consistent with this, a recently published paper reported about synergistic effects of PER1 and SHARP1/2 in circadian period regulation (Bode et al., 2011). 


\subsection{Altered clock gene expression in SHARP1 and -2 single and double null-mutant mice}

To analyse SHARP1 and -2 function in vivo, single and double mutant mice were generated (Rossner et al., 2008). Both single and double mutant mice entrained normally to a 12/12 hour light/dark (LD) cycle. However, under free-running conditions in constant darkness, mice carrying only one or none functional Sharp allele displayed a significant lengthening in period length $(\tau)$ by up to 0.5 hours. This gene dosage dependent effect on $\tau$ revealed a high functional redundancy of both genes. Particular Sharp2 was involved in light entrainment of the SCN as the corresponding single mutant mice showed a reduction of phase shift responses (Rossner et al., 2008). In line with these findings, light induction of the immediate-early genes (IEG) Fos and Perl was attenuated in the SCN in double-null mutant mice, pointing to a direct role of SHARP1 and -2 in circadian gene regulation in the SCN.

Additionally to their function in the SCN, SHARP proteins have also been shown to be involved in clockwork resetting in the forebrain. There, neuronal PAS domain protein 2 (NPAS2) replaces CLOCK functionally (Reick et al., 2001). After exposure to a shifted LD cycle (experimental jet lag of e.g. eight hours delay) single and double mutant mice showed a significantly slower re-entrainment to the novel rhythm, as assessed by wheel-running activity. Again, Sharp2 deficient mice were stronger affected than Sharp1 null-mutant mice (Rossner et al., 2008). Interestingly, mice deficient of NPAS2 have a shorter $\tau$ in DD, are more active during $\mathrm{D}$ and display earlier phase resetting kinetics to an experimental jet lag (Dudley et al., 2003). As NPAS2 is an activator of Clock gene expression, the opposing phenotypes of NPAS2 (transcriptional activator) and SHARP1/2 (transcriptional repressors) deficient mice may hint at antagonizing effects of the transcription factors (Rossner et al., 2008).

When analysing Clock gene expression, SHARP1 and -2 null mutant $\left(\mathrm{S} 1 / 2^{-/}\right)$mice revealed an attenuated Per2 but normal Bmall expression amplitude in the SCN, which was accompanied by a lengthened $\tau$ (Rossner et al., 2008). The link between a dampened Per 2 expression and an increase in $\tau$ was already known for other clock mutant mice (Godinho et al., 2007; Oishi et al., 2000). However, this is in contrast with several in vitro studies describing SHARP1 and -2 exclusively as transcriptional repressors of clock genes (e.g. Per2) (Honma et al., 2002; Nakashima et al., 2008). The facts that tissue-specific functions have already been identified for other clock gene mutations (Oishi et al., 2000; Pando et al., 2002) and that Perl, Per2 and Bmall mRNA amplitudes were known to be elevated in non-SCN 
tissue (cortex and liver) in double mutant mice led to the hypothesis that SHARP1/2 could not only act as transcriptional repressors (cortex and liver) but also be co-activators (in the SCN) in a context-specific manner (Rossner et al., 2008) Moreover, several studies proposed clockwork orange (CWO), the Drosophila homologue of SHARP1 and -2, as a modulator of clock gene expression through both repressing and activating functions (Lim et al., 2007; Richier et al., 2008). As the core clockwork machinery is strongly influenced by the cellspecific transcriptional machinery (Masri and Sassone-Corsi, 2010; Rutter et al., 2002) and as SHARP1 and -2 are known to exert their repressive functions on clock gene transcription via E-box binding (Honma et al., 2002; Li et al., 2004; Nakashima et al., 2008; Sato et al., 2004a) the question arose if SHARP activating function could be dependent on the E-box activator composition. This complex is composed of CLOCK and BMAL1, or NPAS2 and BMAL1 respectively, as NPAS2 replaces CLOCK functionally in the forebrain (Reick et al., 2001).

To conclude, SHARP1 and -2 have been shown to act as adaptation factors of the molecular clock, potentially by exerting repressing and co-activating functions. However, the exact mechanism remained elusive. The current work aimed at getting molecular insight into these speculated dual functions of SHARP1 and -2 proteins.

\subsection{Altered sleep/wake behaviour in SHARP1 and -2 double null-mutant mice}

Sleep is controlled by two systems that operate in part independently of each other: the circadian and the homeostatic (most probably metabolic) mechanisms (Borbély and Achermann, 1999; Franken and Dijk, 2009; Scharf et al., 2008). The circadian system controls the demand for sleep with respect to the 24 hour period and it restricts sleep to an advantageous time of day. The homeostatic mechanisms sense the need for sleep which is constantly increasing during wakefulness. Prolonged wakefulness results in high sleep pressure, cognitive as well as physiological impairments (Van Dongen et al., 2003; Horne, 1988), and can be restored by compensatory or rebound sleep. Long-term sleep deprivation finally leads to death in rats (Cirelli et al., 2006; Rechtschaffen, 1998). Interestingly, disturbed sleep patterns are linked to cognitive dysfunction and mood-related symptoms in psychiatric patients (Diekelmann and Born, 2010; Wulff et al., 2010). Sleep and wakefulness are characterised by distinct cellular gene expression profiles (Cirelli et al., 2004; Mackiewicz et al., 2007) and sleep-dependent brain oscillations might play a role in higher order neuronal 
plasticity (Colgin, 2011; Diekelmann and Born, 2010). About 10\% of expressed genes in the cortex is differentially regulated by day and night (the circadian system) and half of it changes its expression as a function of sleep and wakefulness (behavioural state). These data are consistent with published reports describing that $1-10 \%$ of the transcriptome in the brain and in peripheral organs is controlled by the circadian system (Akhtar et al., 2002; Panda et al., 2002; Storch et al., 2002; Ueda et al., 2002). The canonical circadian gene Per2 for example is influenced both by the time of day and the behavioural state, suggesting a close interplay of the two systems (Cirelli et al., 2004; Wisor et al., 2002).

Before the role of clock genes in sleep regulation is further explained, some basic terms regarding sleep will be introduced. Sleep can be qualitatively as well as quantitatively analysed in humans and rodents by measuring brain electrical activity with the electroencephalogram (EEG). EEG patterns characterise the behavioural state, they change from low voltage and fast spiking during wakefulness and rapid eye movement (REM) sleep to high voltage and slow waves during non rapid eye movement (NREM) sleep. More detailed information regarding sleep quality is given by the EEG power spectrum, which analyses the extent to which specific frequency bands appear in the EEG (Cirelli, 2009). A commonly analysed frequency band is the EEG delta activity (ranging from 0.5-4.5 Hz) during NREM sleep, also termed slow wave activity (SWA), a measure of sleep intensity (Cirelli, 2009).

Additionally to their well known role in generating circadian rhythms, clock genes have been found out to be involved in sleep homeostasis, independent of their circadian function (Franken and Dijk, 2009). However, it is difficult to discriminate between clock and homeostatic effects in mouse models which display a disturbed clock. Also, the functional interplay between the circadian system, the control of sleep and psychiatric diseases still has to be elucidated (Wulff et al., 2010). It is known that Clock mutant mice display hyperactivity, reduced sleep, decreased anxiety and mania-like symptoms accompanied by impaired neuronal synchronisation (Dzirasa et al., 2011; Roybal et al., 2007). Mice deficient for NAPS2, a paralog of CLOCK expressed in the forebrain (Reick et al., 2001), are also hyperactive, show reduced sleep (Dudley et al., 2003) as well as an altered response upon sleep deprivation (SD).

SHARP1 and -2 double mutant $\left(\mathrm{S} 1 / 2^{-/-}\right)$mice reveal functional core clock mechanisms (Bode et al., 2011; Rossner et al., 2008) (see above) and interestingly, SHARP1 was shown to be involved in the regulation of sleep homeostasis in humans (He et al., 2009). A point mutation 
in the C-terminal domain (P385R) of the human SHARP1 gene resulted in a short sleep phenotype. The authors could show that the P385R mutation attenuated the repressive effects of SHARP1 in luciferase reporter gene assays. The short sleep phenotype observed in humans was validated in transgenic mice by using a bacterial artificial chromosome (BAC) clone carrying the mutated human SHARP1. The phenotype was further enhanced when crossing these mice to the SHARP1 null background (He et al., 2009). Sleep architecture in P385R transgenic animals was shown to be more fragmented (especially NREM sleep), whereas wakefulness, NREM and REM sleep were mainly altered during the light phase. P385R transgenic mice also showed a slower recovery upon six hours of sleep deprivation (particularly NREM rebound sleep). SHARP1 null-mutant mice however only displayed a mild sleep phenotype with slightly more sleep in the dark period and slower NREM rebound sleep after SD (He et al., 2009). This strongly indicates a role of SHARP1 in sleep architecture. Due to the functional redundancy of SHARP1 and -2 (Rossner et al., 2008), it was speculated if SHARP2 could compensate at least partially for the SHARP1 loss, which would explain the only moderate sleep phenotype effect in SHARP1 null-mutant mice (Shahmoradi et al., in submission). Therefore, sleep architecture and the response upon SD were studied in S1/2 $2^{--}$mice (Shahmoradi et al., in submission). Total wakefulness, REM and NREM sleep were not altered between mutant and control mice, but when quantified to the light (L) and dark phase (D) separately prominent changes between mutant and control mice could be observed. Whereas as expected, WT mice exhibited a distinct difference in the amount of wakefulness, NREM and REM sleep relative to the L and D (predominately slept during the L phase and kept awake during the dark phase), these effects were attenuated in the mutant mice. This phenotype was accompanied by changes in REM and NREM sleep distribution with a reduction of REM sleep between zeitgeber time (ZT, indicating that light was used as a zeitgber) 4-8 as well as differences in NREM sleep between ZT10-12 (reduced NREM) and ZT19-21 (increased NREM). Moreover, in S1/2 ${ }^{-/-}$mice voluntary running wheel activity was found to be reduced during D, but displayed no changes in L (Shahmoradi et al., in submission).

To further address the homeostatic role of SHARP proteins on sleep, six hours of SD was performed by gentle handling at the beginning of the light period (Shahmoradi et al., in submission). Wakefulness and NREM sleep appeared to be unaltered during the six hours after SD, the relative amount of REM sleep however revealed to be significantly reduced 2-4 hours after SD. SWA, a measure of compensatory rebound sleep, was non significantly elevated in $\mathrm{S} 1 / 2^{-/-}$mice at almost all analysed time points. Moreover, oscillations between 5-9 
$\mathrm{Hz}$ in the theta peak frequency (TPF) were significantly elevated between ZT7-12 in sleep deprived mutant mice (Shahmoradi et al., in submission). To conclude, SHARP1 and -2 transcription factors are involved in regulating sleep homeostasis in the response to SD.

\subsection{Attenuated activity-dependent gene expression in SHARP1 and -2 double null-mutant mice}

Microarray analysis of cortical RNA depicted for two selected time points, ZT4 and ZT16, revealed substantial sleep/wake-related changes in gene expression between WT and $\mathrm{S} 1 / 2^{-/-}$ mice (Shahmoradi et al., in submission). The number of wake-induced transcripts was significantly higher in WT compared to mutant mice, whereas the up-regulation of the core clock gene Per2 was similar between genotypes, indicating normal clock function in mutant mice. Wake-dependent induction of IEGs (e.g. Fos, Nr4a, Egrl, Ierl and Junb) and genes related to inter- and intracellular signalling (e.g. Tac1, Penk, Dusp1/6, Drd2 and Adora2) was completely diminished in mutant mice (Fig. 3). Intriguingly, this cluster of deregulated transcripts contained also oligodendrocyte/myelin specific genes (e.g. Enpp2, Plp1 and Cldn11). This finding of a potential abnormal regulation of myelin genes in $\mathrm{S} 1 / 2^{-/-}$mice was completely unexpected and of high interest, as myelin genes are known to contribute to neural plasticity as well as the pathology of schizophrenia and depression (see below). 


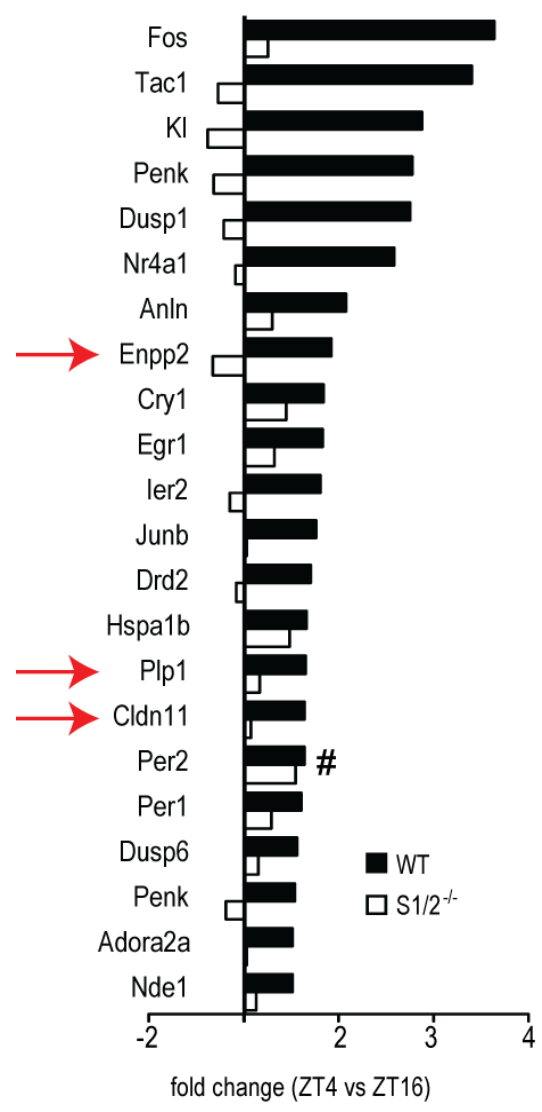

Fig. 3 Attenuated wake-induced cortical gene expression $\mathrm{S} 1 / 2^{-/-}$mice

Depicted are fold-changes of differentially expressed genes at ZT4 and ZT16 in the cortex of WT and $\mathrm{S} 1 / 2^{-/-}$mice detected by microarray analysis. WT mice indicate an up-regulation of several genes by at least factor 1.5 at ZT16, whereas this profile is strongly attenuated in mutant mice. Only Per2 (marked with \#) shows no difference between genotypes. Unexpectedly, myelin genes (indicated by red arrows) appeared among sleep/wake-dysregulated genes in mutant mice. ZT, zeitgeber time; Enpp2, ectonucleotide pyrophosphatase/phosphodiesterase 2; Plp1, proteolipid protein 1; Cln11, claudin 11; Per2, period 2. The figure is taken and modified from Shahmoradi and colleagues (Shamoradi et al., in submission).

\subsection{Myelin and plasticity}

White matter comprises over half of the human brain, it is located beneath the grey matter cortex and consists of millions of bundles of axons (nerve fibres) connecting neurons from different brain regions into important circuits. The white colour deviates from the electrical insulation, myelin, which is wrapped around axons by specialized cells called oligodendrocytes. Myelin plays an important role in high speed transmission of electrical impulses (up to 50-100 times faster condition velocities upon myelination) and is also important for axonal integrity. Alterations in myelin structure and function are associated with severe neurological diseases (Nave, 2010).

In the past years, myelin was regarded with new interest, as it revealed to be a dynamic structure that is modifiable by electrical activity of axons (Zalc and Fields, 2000). Increasing 
evidence suggests that myelin could play an important role in modifying the brain according to experience/environment, thereby contributing to information processing and learning (Fields, 2008). Myelination is considered to be a developmental process, but is known to continue into the third decade of life in humans (Giedd, 2004; Yakovlev and Lecours, 1967) and the development of white matter structures of specific brain regions corresponds to the development of specific cognitive functions (Asato et al., 2010; Mabbott et al., 2006; Nagy et al., 2004) like reading (Kraft et al., 1980) and executive decision making (Giedd, 2004; Liston et al., 2006). Interestingly, weaker decision-making ability and enhanced risk taking have been correlated with insufficient white matter development in the forebrain of adolescents (Beckman, 2004). It has been described over 30 years ago, that in the visual cortex of rats exposed to an enriched environment during adolescence the number oligodendrocytes is increased by 27-33\% (Sirevaag and Greenough, 1987; Szeligo and Leblond, 1977). Environmental enrichment also impacts on the number of myelinated fibres in the corpus callosum of rats (Juraska and Kopcik, 1988; Markham and Greenough, 2004). However, experience-dependent changes of white matter are not restricted to the developmental period (childhood and adolescence). Magnetic resonance imaging (MRI) studies on adult humans revealed a regionally specific plasticity of white matter tracts associated with piano playing (Bengtsson et al., 2005) or after learning a complex visuo-motor task such as juggling (Scholz et al., 2009). Further evidence from studies with human adults who learnt to read (Carreiras et al., 2009) and adult macaque monkeys trained to use tools (Quallo et al., 2009) confirm training-induced changes of white matter structures. However, MRI studies are only an indirect measure of myelination. Histological studies by Markham and colleagues in adult rats housed for two months in an environment revealed a $10 \%$ increase in the size of the corpus callosum. However, this effect was due to an increased capacity of astrocytes and unmyelinated axons (potentially as a result of axon sprouting) and not of newly myelinated axons (Markham et al., 2009). In contrast, the same paradigm in young animals led to an increase of myelinated axons in the corpus callosum (see above) (Juraska and Kopcik, 1988; Markham and Greenough, 2004).

As myelination is known to regulate conduction velocity it can influence the temporal coincidence and the summation of synaptic inputs from distal cortical regions finally resulting in an action potential. The basic mechanism of synaptic plasticity to increase synaptic efficacy depends on the repeated and persistent firing of the presynaptic cell onto the postsynaptic neuron (Hebb's law, often paraphrased as 'neurons that fire together, wire together' (Carla Shatz)). This rule implies that synapses that are coincidently active with postsynaptic neuronal 
firing represent crucial connections that should be strengthened (Fields, 2008). There is an over 100 -fold variability in conduction velocity among axons. $30 \%$ of the fibres in the adult human corpus callosum are unmyelinated, which might be a potential target to myelination. Electrophysiological analysis by chronic recording revealed that conduction velocity is plastic in $1 / 3$ of corpus callosal axons within one year after birth in rabbits (Swadlow, 1985). Myelination regulates the speed of impulse conduction by influencing axon diameter, thickness of myelin sheath, number and spacing of nodes of Ranvier, nodal structure and composition of ion channels in nodal and paranodal region (Fields, 2008).

\subsection{Neurotransmitters implicated in myelination}

Neural activity is known to impact on proliferation and myelination of oligodendrocytes (Demerens et al., 1996; Ishibashi et al., 2006; Stevens and Fields, 2000; Stevens et al., 2002). Oligodendrocyte lineage cells express ion channels, purinergic and other membrane receptors enabling them to sense neuronal activity. Oligodendrocytes also have neurotransmitter receptors for glutamate, serotonin and dopamine (Lee and Fields, 2009). Moreover, synaptic contacts between axons and oligodendrocyte precursor cells (OPCs) in white matter have been found (Bakiri et al., 2009; Kukley et al., 2007; Lin et al., 2005) facilitating communication between these cell types. It has been speculated that many types of neurotransmitters might influence myelination, among them are adenosine 5'-triphosphate (ATP) and glutamate. ATP is described to regulate myelination in response to electrical activity in axons. One published mechanism describes the ATP release from active axons (Fields and Stevens, 2000). In this model, ATP stimulates via purinergic (P)2 receptors on astrocytes the release of leukemia inhibitory factor (LIF), which in turn promotes myelination by oligodendrocytes (Ishibashi et al., 2006). Wake and colleagues showed that electrical activity can influence the induction of myelination at the subcellular level which would allow oligodendrocytes to preferentially myelinate active axons (Wake et al., 2011). The authors found that glutamate released by active axons promotes the formation of lipids rafts in oligodendroglia, thereby activating pathways known to be important for the local translation of $M b p$ mRNA.

\subsection{Myelin and psychiatric disorders}

Another reason why myelination has been regarded with new interest in recent years is its association with psychiatric disorders (Fields, 2008). The current hypothesis regarding the 
development of psychiatric diseases combines alterations in the regulation of many genes which together with multiple environmental risk factors contribute to the illness. Psychiatric diseases have been primarily attributed to synaptic dysfunctions and research mainly concentrated on grey matter. However, recently white matter, especially oligodendrocytes and myelin are brought into focus (Dwork et al., 2007). Polymorphisms for several myelin genes have been linked to the pathology of depression (Tkachev et al., 2003), obsessive-compulsive disorders (Stewart et al., 2007) and schizophrenia (Hakak et al., 2001; Tkachev et al., 2003). Schizophrenia is a severe, chronic psychiatric disorder, influenced by both genetic and environmental factors (Harrison and Weinberger, 2005; Ming, 2000). Cognitive dysfunctions (deficits in attention, working memory and executive function) are a core symptom and have been described as an endophenotype of the disease (Gottesman, 2003). Analysis of post mortem brains from patients suffering from schizophrenia (Georgieva et al., 2006; Tkachev et al., 2003), depression (Aston et al., 2005) and bipolar disorder (Tkachev et al., 2003) revealed a decreased abundance of several mRNA transcripts coding for myelin proteins. Out of 6000 analysed transcripts in the dorsolateral prefrontal cortex (DLPFC) of schizophrenic patients, 89 appeared to be deregulated upon disease, 35 of them being implicated in myelination (Hakak et al., 2001). These initial findings have been validated several times for the DLPFC and additional regions by different techniques (Table 1). 
Table 1 Oligodendrocyte and myelin-related genes in schizophrenia Summary of oligodendrocyte and myelin-related genes implicated in schizophrenia from gene expression and genetic association studies. CNV, copy number variation. The figure is taken from Takahashi and colleagues (Takahashi et al., 2011).

\begin{tabular}{|c|c|c|}
\hline Gene symbol & $\begin{array}{l}\text { Altered gene expression } \\
\text { (major or first study) }\end{array}$ & $\begin{array}{l}\text { Genetic association } \\
\text { (major or first study) }\end{array}$ \\
\hline CLDN11 & Tkachev et al. (2003) & \\
\hline CNP1 & Hakak et al. (2001) & Peirce et al. (2006) \\
\hline DISC1 & & Millar et al. (2000) \\
\hline ERBB3 & Tkachev et al. (2003) & \\
\hline ERBB4 & & $\begin{array}{l}\text { Norton et al. (2006), } \\
\text { Walsh et al. }(2008)^{a}\end{array}$ \\
\hline MAG & Tkachev et al. (2003) & $\begin{array}{l}\text { Wan et al. (2005), } \\
\text { Yang et al. (2005) }\end{array}$ \\
\hline MAL & Hakak et al. (2001) & \\
\hline MBP & Tkachev et al. (2003) & \\
\hline МOBР & Tkachev et al. (2003) & \\
\hline MOG & Tkachev et al. (2003) & Liu et al. (2005) \\
\hline NRG1 & & $\begin{array}{l}\text { Stefansson et al. (2002), } \\
\text { Williams et al. (2003) }\end{array}$ \\
\hline Olig2 & Tkachev et al. (2003) & Georgieva et al. (2006) \\
\hline PLP1 & Tkachev et al. (2003) & Qin et al. (2005) \\
\hline PMP22 & Dracheva et al. (2006) & Kirov et al. (2009) ${ }^{a}$ \\
\hline PTPRZ1 & & Buxbaum et al. (2008) \\
\hline QKI & & Lindholm et al. (2001) \\
\hline RTN (NOGO) & & $\begin{array}{l}\text { Budel et al. }(2008)^{\mathrm{b}} \text {, } \\
\text { Kirov et al. }(2009)^{\mathrm{a}}\end{array}$ \\
\hline SOX10 & Tkachev et al. (2003) & Maeno et al. (2007) \\
\hline $\mathrm{TF}$ & Hakak et al. (2001) & Qu et al. (2008) \\
\hline
\end{tabular}

The group of deregulated transcripts encompasses genes coding for structural myelin proteins (Mag, Mal, Mbp , Plp and Cnp), genes encoding transcription factors (Sox10, Olig1 and Olig2) as well as for growth factors and receptors like $\mathrm{ErbB3}, \mathrm{Nrgl}$ and Bdnf. The biggest changes were seen in the cingulate cortex, the superior temporal gyrus and the hippocampus. Despite that only a few studies analysed alterations on protein level, it is known that CNPase is reduced in the prefrontal cortex (Flynn et al., 2003).

It is currently under debate if these changes in white matter are caused by alterations in number, densities and spacing of oligogendrocytes. A study by Stark (Stark et al., 2004) revealed a decrease in oligodendrocyte density specific to cingulate area 24 and not involving the surrounding area 32. Similarly, a decreased oligodendrocyte number was observed in prefrontal area nine of the superior frontal gyrus (Hof et al., 2003), but not in the anterior cingulum bundle (Segal et al., 2009), pointing towards more sublte oligodendrocyte and myelin abnormalities causing the observed structural defects in the cingulum bundle (Höistad et al., 2009). However, electron microscopy studies of oligodendrocytes in the PFC showed apoptosis and mitochondria abnormalities as well as damaged myelin in area ten in schizophrenic patients (Uranova et al., 2001, 2004). 
Diffusion tensor imaging (DTI) has been emerged as an important non invasive technique to study white matter structures in vivo, which gives a great opportunity to complete post mortem data. Recently, many DTI analyses have been published showing a decreased fractional anisotropy (FA, a measure of white matter integrity) in schizophrenic brains, particularly in the prefrontal, temporal, parietal and occipital lobes (Heimer, 2000; Kanaan et al., 2005; Kubicki et al., 2007) as well as the corpus callosum (Hulshoff Pol et al., 2004). A meta-analysis revealed $1 \%$ reduction of whole brain white matter upon schizophrenia (Wright et al., 2000). White matter alterations have been discussed to cause the disconnectivity of neuronal circuits ('disconnection hypothesis' by Friston (Friston, 1999)) potentially contributing to the development of cognitive dysfunction in schizophrenic patients due to dysfunctional integration. Intriguingly, a reduction in white matter volume is already investigated in subjects being at ultra high risk to develop schizophrenia (showing first psychotic symptoms), meaning that myelin alterations represent a primary defect of the illness, which might precede the disease (Witthaus et al., 2008).

A tightly regulated myelination process plays an indispensable role for functional information processing between distant regions of the brain (Fields, 2008). Alterations in myelin have been reported in schizophrenia (Thaker, 2008), ultimately contributing to disorganized thought and cognitive dysfunctions accompanied with the disease (Tanaka et al., 2009). Evidence for this hypothesis comes from animals models of schizophrenia, for instance Plp transgenic mice. Proteolipid protein (PLP) is a highly abundant tetraspanning myelin protein. Plp transgenic mice reveal a reduction in conduction velocity accompanied by cognitive deficits in prepulse inhibition (PPI) test as well as deficits in working and spatial memory (Tanaka et al., 2009). Another example comes from ErbB4 mutant mice. ErbB4 is a receptor tyrosine kinase, which can be activated by Neuregulin-1 (Nave and Salzer, 2006), one of the crucial modulators of myelination in the peripheral nervous system (PNS) (Michailov et al., 2004). Mice expressing a dominant negative from of ErbB4 in oligodendrocytes display slower conduction velocities and behavioural alterations reminiscent of psychiatric disorders like enhanced amphetamine sensitisation (Roy et al., 2007). Further, these mice display an increased abundance of dopamine receptors and transporters in several brain areas (Roy et al., 2007).

Regarding the pathophysiology of schizophrenia two hypotheses are speculated: the dopamine and the glutamate hypothesis. 


\section{Dopamine hypothesis}

Supporting for the dopamine hypothesis is the fact that all available antipsychotic drugs block the dopamine receptor D2 (DRD2). This provides evidence that dopaminergic signalling may be involved in the pathophysiology of schizophrenia (Takahashi et al., 2011). Enhanced dopaminergic activity in the striatum may precede the beginning of the disease (Howes et al., 2009) and has been linked to hypo-prefrontal function associated with schizophrenia (FusarPoli et al., 2011). Recent evidence suggests that oligodendrocyte and myelin dysfunction might contribute to alterations in dopamine signalling. Patients suffering from multiple sclerosis (MS) display psychiatric symptoms, which can be ameliorated with neuroleptics (Safferman et al., 1994; Takahashi et al., 2011). In line with this, mice treated with cuprizone, an agent inducing demyelination, displayed an increased dopamine concentration in the PFC mice (Xu et al., 2009). These animals showed altered behaviour which is associated to changes in dopamine levels, such as reduced working memory and deficits in sensorimotor gating (Geyer, 2008).

\section{Glutamate hypothesis}

Glutamate signalling may also play a role in the pathology of schizophrenia. Pharmacological inhibition of glutamatergic N-methyl-D-aspartate (NMDA) receptors by phencyclidine (PCP) caused schizophrenia-like symptoms in humans (Javitt and Zukin, 1991). Glutamate levels were found to be reduced in the cerebrospinal fluid (CSF) in schizophrenic patients (Kim et al., 1980). Post mortem analysis revealed changes in glutamate receptor binding, transcription and subunit composition in the PFC and hippocampus in schizophrenics (Clinton and Meador-Woodruff, 2004). Moreover, the concentration of N-acetylaspartate (NAA) was changed in the CSF and tissue in a disease-related manner (Tsai and Coyle, 1995). Microarray analysis indicated the deregulation of key enzymes in the NAA metabolism, leading to a reduced production of myelin lipids due to a lack of acetate levels and also to reduced glutamate concentration (Tkachev et al., 2007).

In the present study we further analysed preliminary findings of a potential circadian myelin gene expression in WT and a deregulation in $\mathrm{S} 1 / 2^{-/-}$mice. A dynamic regulation of myelin genes in the adult would be of high interest with respect to the brain's plasticity to environmental influence. An altered expression of these genes in a mouse model in which 1. the homeostatic control of sleep is changed and 2. which might be associated to psychiatric 
diseases could provide new insights regarding the complex pathophysiology of these disorders. 


\section{AIM OF THE STUDY}

Previous analyses provided a link between endophenotypes of psychiatric disorders and the attenuated wake-induced expression of cortical transcripts in SHARP1 and -2 double mutant $\left(\mathrm{S} 1 / 2^{-/}\right)$mice. Moreover, among sleep/wake dysregulated genes, transcripts encoding myelin proteins were identified. This finding was of particular interest as it is known that myelin genes are deregulated in psychiatric diseases.

This prompted us to perform behavioural analyses of $\mathrm{S} 1 / 2^{-/-}$mice with respect to motivation-, exploratory-, curiosity- and anxiety-related behaviour to identify potential phenotypes reminiscent of psychiatric disorders. Therefore, these studies would further support the view of $\mathrm{S} 1 / 2^{-/-}$mice as a model for psychiatric diseases. Also, the potential dynamic regulation of myelin genes in WT mice and the attenuated effects observed in the mutant mice should be further addressed. We planned to combine laser-captured microdissection with quantitative RT-PCR to analyse day time-dependent myelin gene expression in precisely defined grey and white matter micro-regions. Profiles resulting from these experiments should be compared with CNS and PNS nerves to elucidate region-specific effects.

To approach the homeostatic role of SHARP proteins associated with sleep, we planned to perform sleep deprivation in control and $\mathrm{S} 1 / 2^{-/-}$mice to further study potential effects on myelin gene expression. In vitro experiments in primary cultured oligodendrocytes of WT and mutant mice were aimed to corroborate potential genotype-specific differences in myelin gene regulation, particularly in response to stimulated conditions.

Finally, to address mechanisms, we planned to conduct reporter gene assays in various heterologous cell lines as well as primary cultured neurons to study context-dependent functions of SHARP proteins. In the end, reporter gene assays in the oligodendroglial precursor cell line Oli-neu and in primary cultured oligodendrocytes should elucidate potential effects of SHARP proteins on myelin gene promoters. 


\section{Results}

\subsection{Behavioural analyses}

\subsubsection{Standard behavioural tests}

To assess if SHARP1 and -2 mutant $\left(\mathrm{S} 1 / 2^{-/-}\right)$mice display any behavioural alterations regarding activity, curiosity, fear, motivation and pain sensitivity a series of standard behavioural analyses was performed. These tests included the open field test, hole board test, tail suspension test and hot plate test. Taken together, this analysis could reveal a potentially altered behavioural phenotype of $\mathrm{S} 1 / 2^{-/-}$mice that may be particularly relevant in the context of psychiatric diseases.

\subsubsection{Increased activity and reduced anxiety levels in $S 1 / 2^{-/-}$mice in the open field test}

The open field test (Broadhurst, 1961; Hall, 1934) is a commonly used test to assay spontaneous motor activity and anxiety of mice. The parameters 'travelled distance' and the 'time spent active' provide information about the general locomotor activity of mice. The time an animal spent in the 'dangerous' centre and in the 'secure' corners of the open field allows conclusions about anxiety.

In all analysed parameters $\mathrm{S} 1 / 2^{-/-}$mice displayed highly significant differences in comparison to wild type (WT) mice (Fig. 4A-D). WT animals $(\mathrm{n}=24)$ travelled on average $28.1 \mathrm{~m}$, mutant mice $(n=26)$ covered a distance of $35.3 \mathrm{~m}(\mathrm{p}=0.0006)$ (Fig. 4A; mean values with SEM in the bar graph; mean values including individual mice in the dot plot). Accordingly, mutant mice were active $54.7 \%$ of the total time, whereas the value for control mice was $45.6 \%$ of the total time $(\mathrm{p}<0.0001)$ (Fig. 4B). Control mice spent $12 \%$ of the time in the centre $(70 \%$ of the open field arena) (Fig. 4C) and remained $70.1 \%$ of the total time in the corners of the arena (Fig. $4 \mathrm{D})$. The corresponding values for the mutant mice were $28 \%$ for the centre $(\mathrm{p}=0.0004)$, and $53.8 \%$ for the corners $(p<0.0001)$. 
A

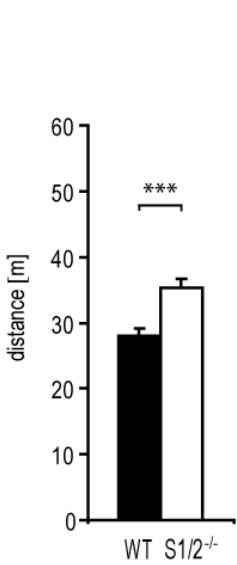

Open field

distance

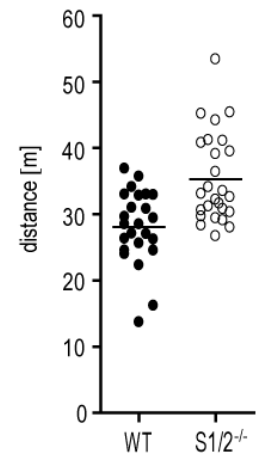

Open field

time in centre
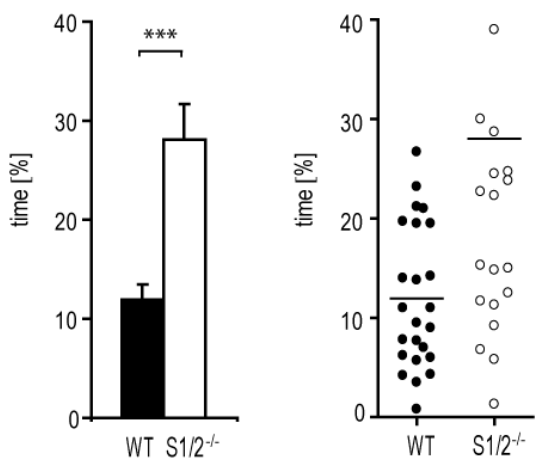

B

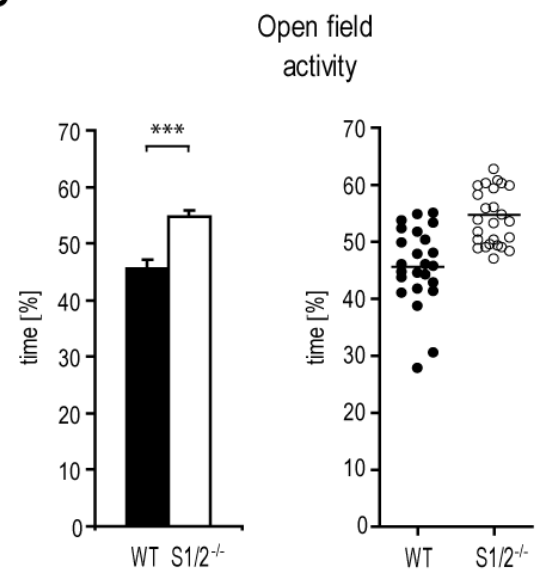

D

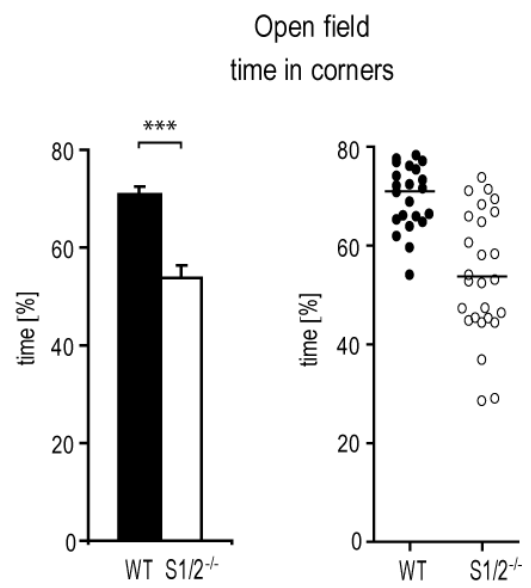

Fig. 4 Increased activity and reduced anxiety levels in $\mathrm{S} 1 / 2^{-/-}$mice in the open field test (A) $\mathrm{S} 1 / 2^{-/-}$mice travelled a longer distance $(\mathrm{p}=0.0006)$ and $(\mathrm{B})$ spent more time being active $(\mathrm{p}<0.0001)$ compared to controls. (C) $\mathrm{S} 1 / 2^{-/-}$mice stayed a significantly longer time in the centre of the box $(p=0.0004)$, and (D) less time in the corners than WT animals $(p<0.0001)$. Data represent mean values \pm SEM for bar graphs and mean values with individual animals in the scatter plots for $n=24$ WT and $n=26$ $\mathrm{S} 1 / 2^{-/}$mice. Only male animals at the age of four months were used in the experiments. $* * *=\mathrm{p}<0.001$. Significance values refer to the Mann-Whitney test. WT, wild type; S1/2/-, SHARP1 and -2 double nullmutant; SEM, standard error of the mean.

\subsubsection{Decreased curiosity of $S 1 / 2^{-/-}$mice in the hole board test}

For the hole board test (Bradley et al., 1968), a modified open field box was used where an additional board containing 16 holes was inserted. The test allows to monitor the curiosity of mice, which is scored by nose pokes into the holes. WT and mutant mice showed no significant difference in the covered distance, with $20.7 \mathrm{~m}$ for WT and $21.8 \mathrm{~m}$ for S1/2 ${ }^{-/-}$mice $(\mathrm{p}=0.4269)$ (Fig. 5A; mean values with SEM in the bar graph; mean values including individual mice in the dot plot). Significant differences between the genotypes were found for the number of hole visits (nose pokes), with $\mathrm{p}=0.0015$ (Fig. 5B) and the total hole exploration time, with $\mathrm{p}=0.0026$ (Fig. 5C). WT mice revealed on average 22.3 nose pokes, and a total 
hole exploration time of $26.9 \mathrm{~s}$. The corresponding readings for $\mathrm{S} 1 / 2^{-/-}$mice were 11.6 nose pokes and $14.6 \mathrm{~s}$ total hole exploration time. The average exploration time per hole was $1.21 \mathrm{~s}$ for WT and $1.25 \mathrm{~s}$ for mutant mice.

A

Hole board

distance

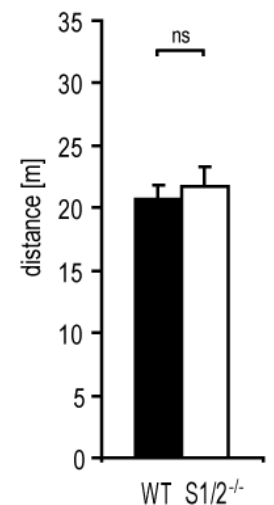

C

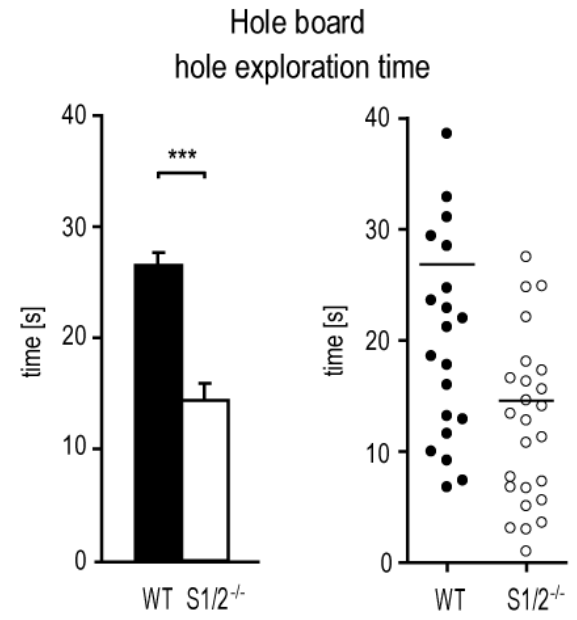

B

Hole board

number of visits
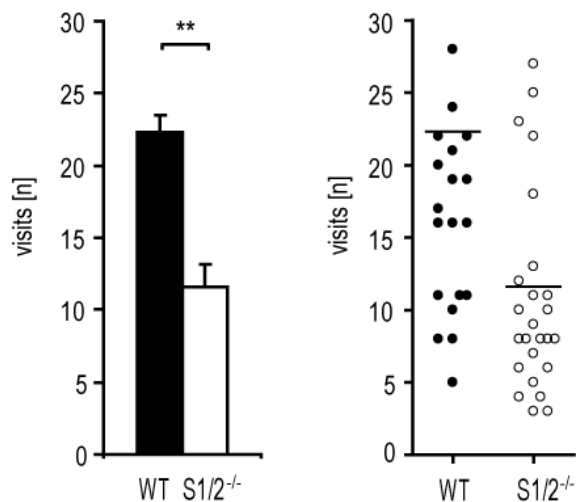

Fig. 5 Decreased curiosity of $\mathrm{S} 1 / 2^{-/-}$mice in the hole board test

(A) $\mathrm{S} 1 / 2^{-/-}$mice showed no significant difference in the travelled distance $(\mathrm{p}=0.4269)$ but (B) decreased curiosity behaviour as indicated by a significantly smaller number of hole visits $(\mathrm{p}=0.0015)$ and $(\mathrm{C})$ a decreased hole exploration time. Data represent mean values \pm SEM for bar graphs and mean values with individual animals in the scatter plots for $n=24 \mathrm{WT}$ and $n=26 \mathrm{~S} 1 / 2^{-/-}$mice. All analysed animals were male and four months of age. Ns, non significant; $* *=\mathrm{p}<0.01 ; * * *=\mathrm{p}<0.001$. Significance values refer to the Mann-Whitney test. WT, wild type; S1/2 ${ }^{-/}$, SHARP1 and -2 double null-mutant; SEM, standard error of the mean.

\subsubsection{No differences in sensory processing in $S 1 / 2^{-/-}$mice in the hot plate test}

The hot plate test (O’Callaghan and Holtzman, 1975) was performed to compare sensory processing (pain sensitivity) of $\mathrm{S} 1 / 2^{-/-}$mutant mice to WT controls. 
$17 \mathrm{WT}$ and $13 \mathrm{~S} 1 / 2^{-/-}$adult male mice (four months of age) were subsequently placed on a $55^{\circ} \mathrm{C}$ hot plate, and the latency to show the first reaction of discomfort, licking the hind limb or jumping, was analysed (Fig. 6A; mean values with SEM in the bar graph; mean values including individual mice in the dot plot). WT animals started licking the hind paws after 18.5 $\mathrm{s}$, whereas the mutant mice showed this reaction after $21 \mathrm{~s}$. No significant difference between the genotypes could be detected $(\mathrm{p}=0.8050)$. In conclusion, mutant mice showed similar pain sensitivity as controls did.

\subsubsection{Tendency towards increased motivational behaviour of $S 1 / 2^{-/-}$mice in the tail suspension test}

The tail suspension test measures motivational behaviour of mice in terms of efforts to escape from an uncomfortable situation (Steru et al., 1985). In this test $17 \mathrm{WT}$ and $13 \mathrm{~S} 1 / 2^{-/}$adult male mice were analysed (Fig. 6B; mean values with SEM in the bar graph; mean values including individual mice in the dot plot). Spending in average $46.1 \%$ of total time moving, $\mathrm{S} 1 / 2^{-/-}$mutant mice displayed a non significant increase in activity $(\mathrm{p}=0.1216)$ in comparison to WT mice with $36.6 \%$ activity, which might suggest slightly increased motivation. 
A

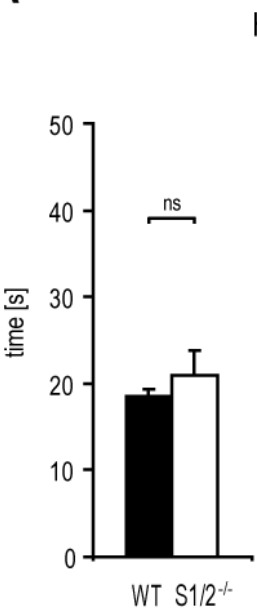

Hot plate

time

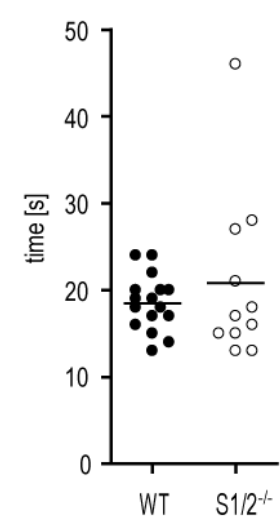

B

Tail suspension

time active

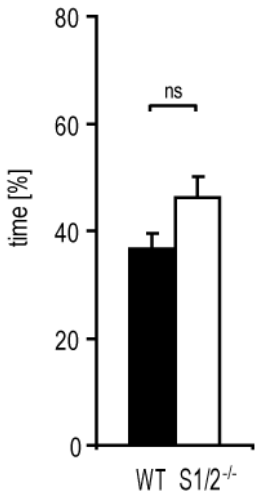

Fig. 6 No differences in sensory processing in $S 1 / 2^{-/-}$mice in the hot plate test and a tendency towards increased motivational behaviour in the tail suspension test

(A) No difference in pain sensitivity and sensory processing between WT and $\mathrm{S} 1 / 2^{-/}$mice could be detected in the hot plate test ( $\mathrm{p}=08050)$. (B) $\mathrm{S} 1 / 2^{-/ /}$mice displayed a non significant tendency $(\mathrm{p}=0.1216)$ to enhanced escape behaviour of an uncomfortable situation in the tail suspension test. Data represent mean values \pm SEM for bar graphs and mean values with individual animals in the scatter plots for $\mathrm{n}=17$ WT and $n=13 \mathrm{~S} 1 / 2^{-/-}$mice. All analysed animals were male and four months of age. Ns, non significant. Significance values refer to the Mann-Whitney test. WT, wild type; S1/2 ${ }^{-/}$, SHARP1 and -2 double nullmutant; SEM, standard error of the mean.

\subsection{Dynamic regulation of myelin genes in precise grey and white matter micro-areas of the brain}

One aim of this thesis was to analyse day time-dependent myelin gene expression in precisely defined micro-areas of the cortex and corpus callosum in adult $\mathrm{WT}$ and $\mathrm{S} 1 / 2^{-/-}$mice. Thus, laser-captured microdissection (LCM) was combined with quantitative RT-PCR (qRT-PCR). To compare gene expression profiles in the brain with central (CNS) and peripheral nervous system (PNS) nerves, the optic and the sciatic nerve were also investigated (Fig. 7). 


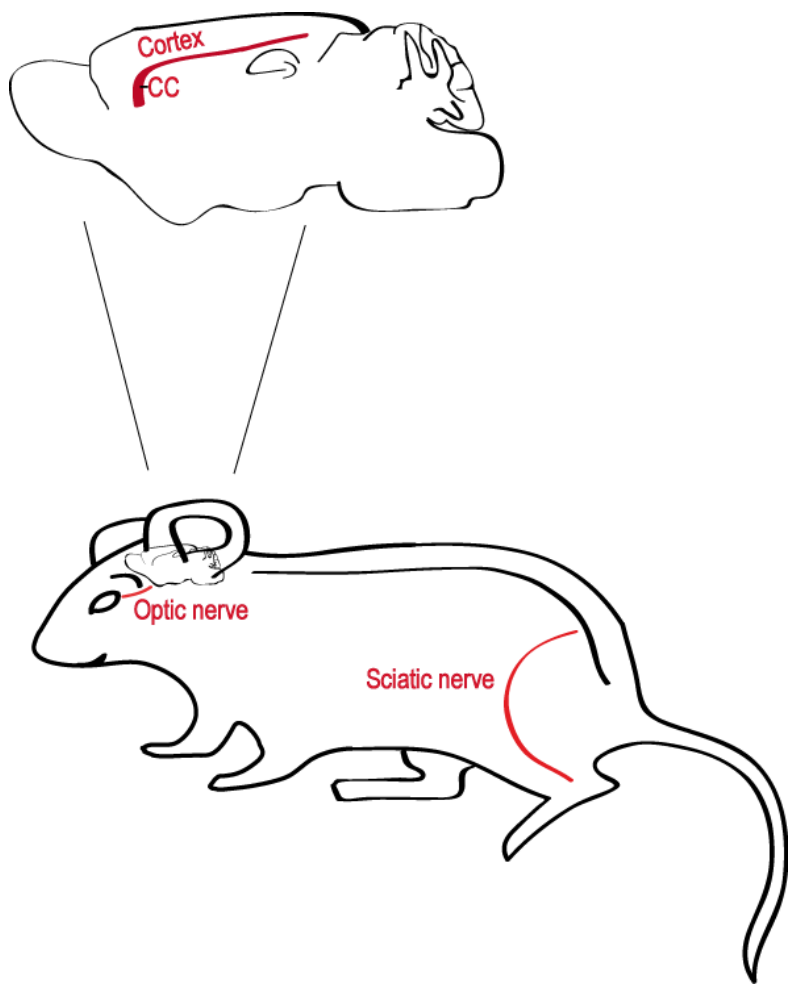

Fig. 7 Schematic representation of the brain areas and nerves taken for analysis

Distinct micro-areas of the cortex and the corpus callosum (both tissues indicated in red, see magnification of the brain) were isolated with LCM and analysed by qRT-PCR. A nerve from the CNS (optic nerve) and the PNS (sciatic nerve, both represented in red) were additionally taken for gene expression analysis. CC, corpus callosum; LCM, laser-captured microdissection; CNS, central nervous system; PNS, peripheral nervous system.

Adult (four months of age), male mice were exposed to an enriched environment for two weeks to enhance sensory, motor and cognitive stimulation in the brain (Nithianantharajah and Hannan, 2006) (Fig. 8A). Mice were sacrificed at four different circadian time points at zeitgeber time (ZT) 4, 10, 16 and 22 with $\mathrm{n}=3$ for each genotype and time point (Fig. 8B). 
A

B

12/12 Hour Light/Dark Cycle

ZTO 12

24
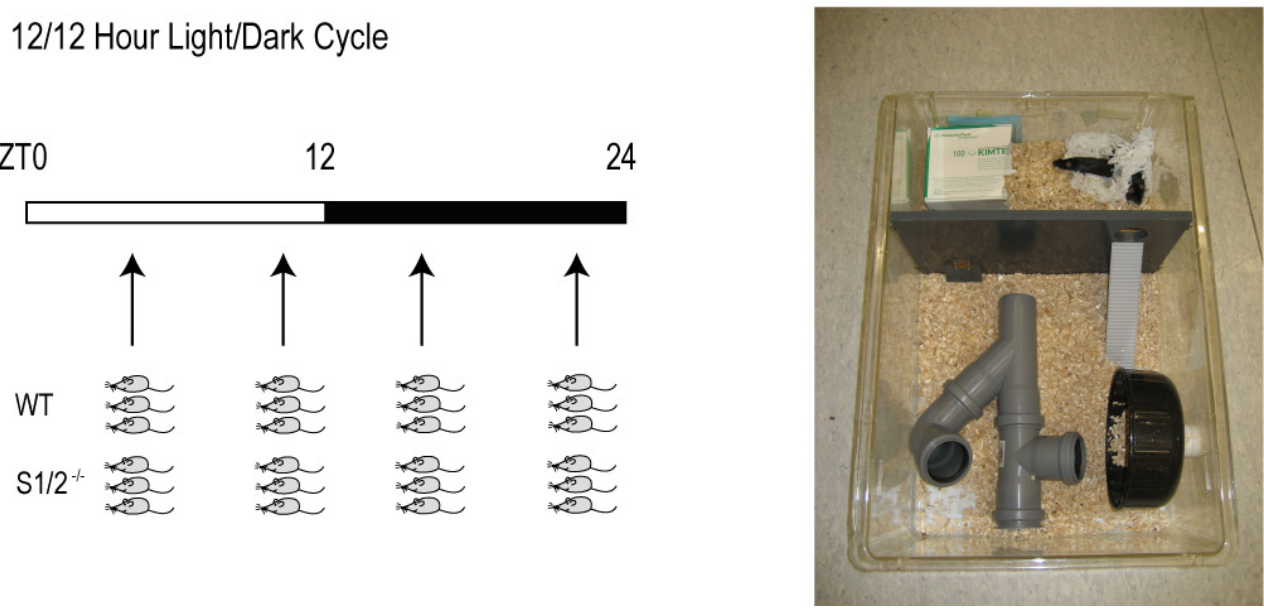

Fig. 8 Experimental design: Housing conditions and analysed time points

(A) Adult (four months of age) male WT and S1/2/- mice were entrained to a $12 / 12$ hour LD cycle and (B) exposed to an enriched environment for two weeks. Afterwards, animals were sacrificed at four different circadian time points: ZT4, 10, 16 and 22 with $\mathrm{n}=3$ for each genotype and time point. Brains were immediately frozen on dry ice to preserve RNA integrity and further subjected to cryosectioning for LCM. Corresponding animals were pooled for further analysis. WT, wild type, S1/2 ${ }^{-/}$, SHARP1 and -2 double mutant; ZT, zeitgeber time; LD, light/dark; LCM, laser-captured microdissection.

Using LCM, precise micro-areas of the cortex and corpus callosum at Bregma $-0.7 \mathrm{~mm}$ were isolated (Fig. 9). Corresponding animals were pooled for further analysis. 
A

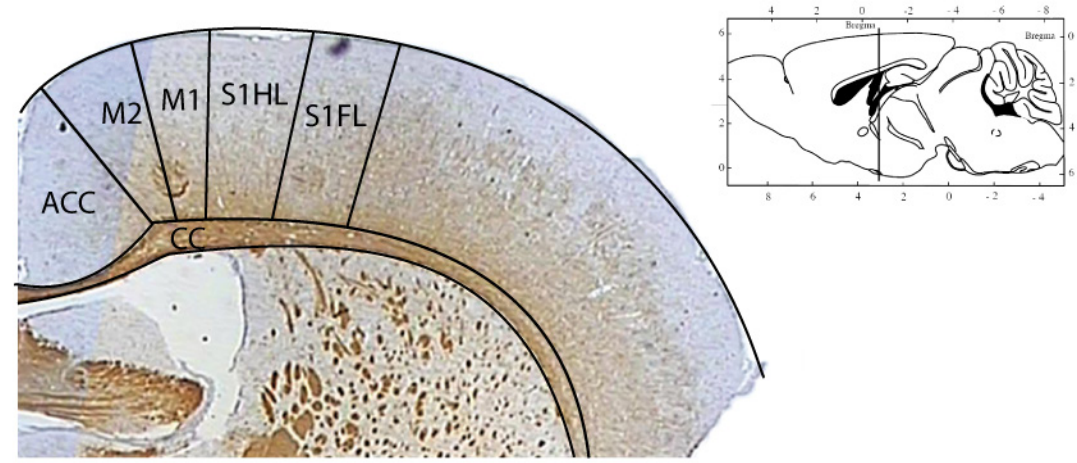

B

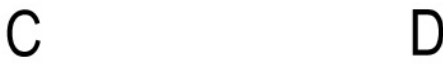

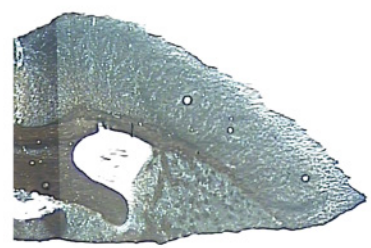

Section on PEN slide

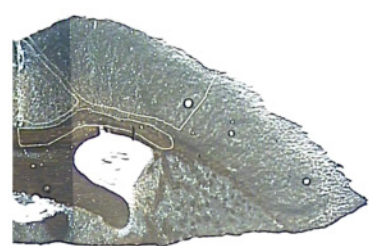

Micro-areas cut
D

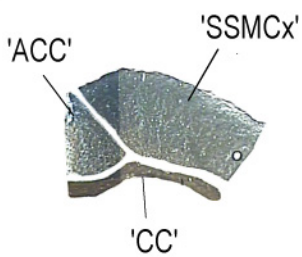

Micro-areas on cap

Fig. 9 Overview of selected micro-areas

(A) CNPase staining visualising myelin in the cortex and corpus callosum at Bregma $-0.7 \mathrm{~mm}$ with indicated subcortical regions. (B) Overview of a section on a PEN slide before LCM, (C) after LCM and (D) selected micro-areas 'ACC', 'SSMCX' and 'CC' on a cap. ACC, anterior cingulate cortex; M1/2, primary motor cortices $1 / 2$; S1HL, somatosensory cortices 1 hindlimb; S1FL, somatosensory cortices 1 forelimb; CC, corpus callosum; CNPase, 2',3'-cyclic-nuleotide 3'-phosphodiensterase; LCM, lasercaptured microdissection.

Table 2 Overview micro-regions and description

\begin{tabular}{|l|l||}
\hline \multicolumn{1}{|c|}{ Micro-region } & \multicolumn{1}{c|}{ Description } \\
\hline \hline ACC & Micro-region of the anterior cingulate cortex \\
\hline & $\begin{array}{l}\text { Micro-region of the somatosensory motor cortex, somatosensory cortices } \\
1 \text { hind limb region and forelimb region as well as primary motor cortices } \\
1 \text { and } 2\end{array}$ \\
\hline CC & Micro-region of the corpus callosum \\
\hline
\end{tabular}

\subsubsection{Circadian and activity-dependent regulation of myelin genes in the ACC micro- area in WT mice and attenuated effects in $\mathrm{S} 1 / 2^{-/-}$mice}

qRT-PCR data were normalised to the housekeeping genes Atp5b and Cycl (Fig. 10A,B). Atp5b encodes the $\beta$ subunit of the mitochondrial ATP synthase, whereas $C y c 1$ codes for a subunit of the electron transport chain protein ubiquitinol cytochrome $\mathrm{C}$ reductase. Atp $5 b$ and 
Cycl expression levels appeared to be most stable among several reference genes. To ensure both optimal technical and experimental performance Per2 and Fos gene expression levels were always analysed first (Fig. 10C,D). The canonical circadian gene Per2 is regulated both by time and activity (Cirelli et al., 2004), and it is supposed be higher expressed in the dark ('activity') phase than in the light ('rest') period. Fos belongs to the family of immediateearly genes (IEG), and together with Jun, it forms the AP1 transcription factor complex. Upregulation of Fos mRNA is considered as a marker for neuronal activity (Bullitt, 1990; Dragunow and Faull, 1989), and thus shows higher expression levels during the dark phase in mice.

Per2 levels slowly increased from ZT4 to ZT10 and then stronger to the peak of expression at ZT16 (factor 4.1 for WT and 3 for S1/2/- mice) (Fig. 10C). After ZT16 the amount of mRNA constantly decreased to the level of ZT4. The pattern of regulation was very similar between WT and mutant mice, although the level appeared to be slightly elevated in the mutant mice throughout the monitored time. Two-way analysis of variance (ANOVA) revealed a significant effect for the interaction of genotype $\left(\mathrm{WT} / \mathrm{S} 1 / 2^{-/-}\right)$and time $\left(\mathrm{F}_{(3,12)}=13.56\right.$, with $\mathrm{p}<0.0004)$.

Fos expression was strongly induced from ZT10 to ZT16/ZT22 in both genotypes, showing a peak in the dark phase, which is consistent with the literature (see above) (Fig. 10D). In the WT situation, Fos levels were slightly decreasing from ZT4 to ZT10, but followed by strong activation during the dark phase, with a peak at ZT22 (8.3-fold induction from peak to trough) and a concomitant down-regulation until ZT4. As mentioned above, Fos expression in the mutant mice very much resembled the WT profile, but the mRNA concentration was generally higher. The maximum of regulation from the trough at ZT10 to the peak at ZT16 was 4-fold. Two-way ANOVA showed a significant effect for the interaction of genotype $\left(\mathrm{WT} / \mathrm{S} 1 / 2^{-/-}\right)$and time $\left(\mathrm{F}_{(3,12)}=76.56\right.$, with $\left.\mathrm{p}>0.0001\right)$. As the expression profiles for the technical control genes Per2 and Fos were consistent with the literature, myelin genes of interest were further analysed.

In WT mice, 2',3'-cyclic-nuleotide 3'-phosphodiensterase (Cnp) only displayed a weak regulation with constant levels of expression from ZT4 to ZT16 (Fig. 10E). At ZT22 a mild trough could be observed (1.25-fold decrease). The mutant mice showed a very similar regulation for $C n p$, with marginally enhanced expression from ZT4 to ZT10 followed by decreasing levels to ZT22. Of note, Cnp expression was slightly higher in the mutant mice compared to the knockout mice at all indicated time points. Two-way ANOVA revealed a non 
significant effect of the interaction of genotype $\left(\mathrm{WT} / \mathrm{S} 1 / 2^{-/-}\right)$and time $\left(\mathrm{F}_{(3,13)}=0.91\right.$, with $\mathrm{p}>0.05)$.

In WT animals, proteolipid protein (Plp) mRNA abundance slightly dropped from ZT4 to ZT10, followed by an increase at ZT16 (factor 1.6), which is indicated by the peak (Fig. 10F). In the mutant mice, $P l p$ only showed minor variability in expression, whereas the levels were comparable to those of WT mice. Two-way ANOVA revealed a significant effect of the interaction of genotype $\left(\mathrm{WT} / \mathrm{S} 1 / 2^{-/-}\right)$and time $\left(\mathrm{F}_{(3,13)}=29.48\right.$, with $\left.\mathrm{p}<0.0001\right)$.

Myelin and lymphocyte protein (Mal) expression also peaked at ZT16 in WT mice, and the mode of regulation resembled that of Plp (Fig. 10G). From ZT4 to ZT10, the expression curve slightly dropped to the bottom peak at ZT10, and then rose 2-fold to the peak at ZT16. Afterwards, the expression again declined. In S1/2/-- mice, the degree of regulation appeared to be attenuated, the diagrams show a trough at ZT16 and a mild peak at ZT22 (factor 1.5). On average, Mal appears to be lower expressed in mutant mice. Two-way ANOVA revealed a significant effect of the interaction of genotype $\left(\mathrm{WT} / \mathrm{S} 1 / 2^{-/-}\right)$and time $\left(\mathrm{F}_{(3,13)}=123.56\right.$, with $\mathrm{p}<0.0001)$.

For myelin basic protein (Mbp), expression levels in WT mice showed a trough at ZT10 followed by the highest abundance at ZT16 (2.3-fold increase) (Fig. 10H). The regulation in the mutant mice indicated almost no variability. Compared to WT mice, Mbp was higher expressed in the mutants. Two-way ANOVA revealed a significant effect of the interaction of genotype $\left(\mathrm{WT} / \mathrm{S} 1 / 2^{-/}\right)$and time $\left(\mathrm{F}_{(3,13)}=103.40\right.$, with $\left.\mathrm{p}<0.0001\right)$.

To draw a short conclusion, in the ACC micro-area myelin genes in WT mice revealed to be dynamically regulated over time, following Per2 and Fos expression (except for Cnp). In $\mathrm{S} 1 / 2^{-/}$mutant mice, this regulation appeared to be attenuated (except for Cnp). 


\section{Reference genes}

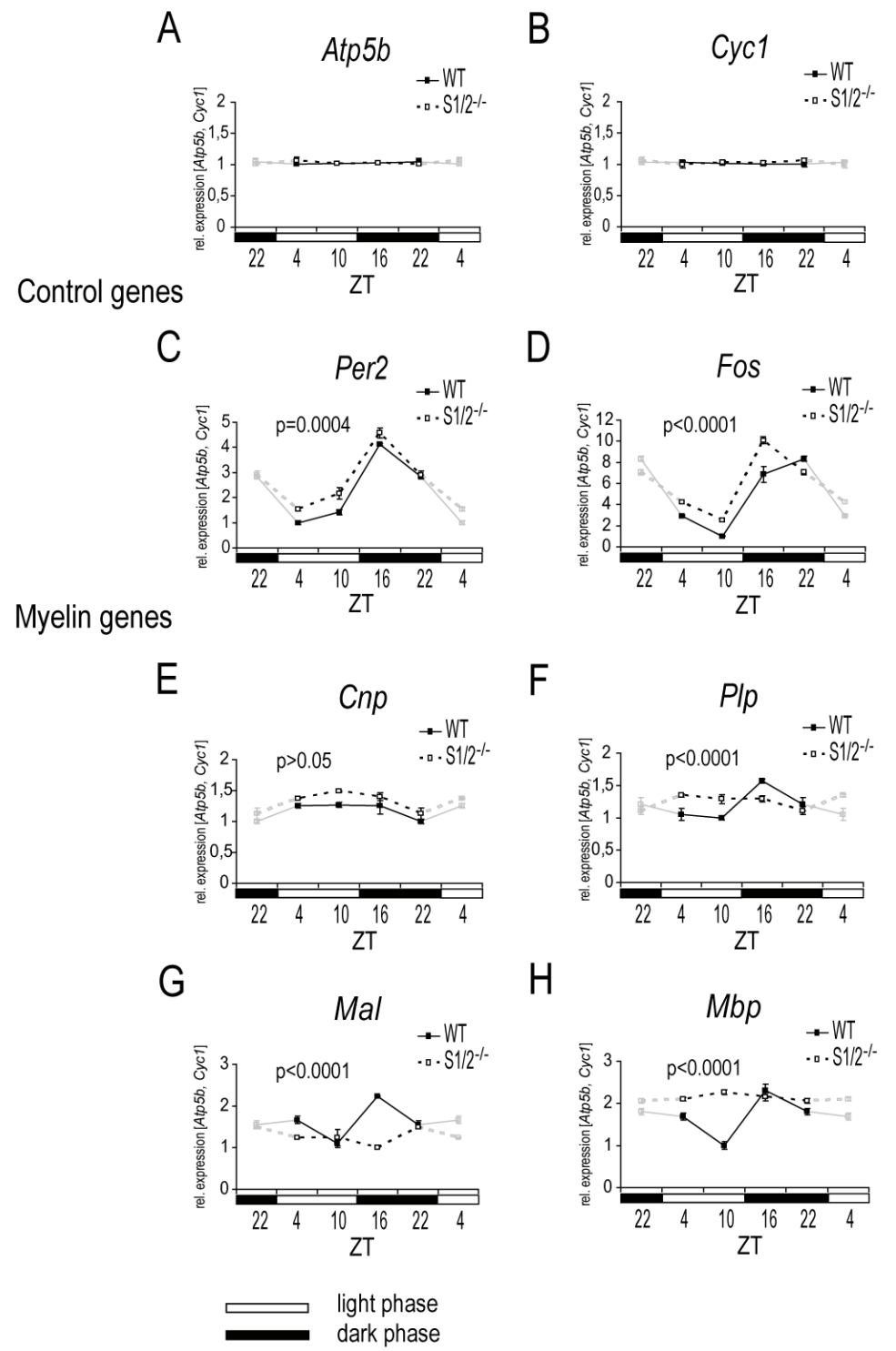

Fig. 10 Circadian and activity-dependent regulation of myelin genes in the ACC micro-area in WT mice and attenuated effects in $\mathrm{S} 1 / 2^{-/-}$mice

Gene expression analysis was performed by qRT-PCR. (A,B) Expression levels were normalised to the housekeeping genes Atp5b and $C y c 1$, which both revealed stable profiles. (C) Per2 expression was as expected up-regulated in both WT and $\mathrm{S} 1 / 2^{-/}$mice at ZT16, which indicated optimal experimental performance. Two-way ANOVA showed a significant result for the interaction of genotype (WT/S1/2--) and time $\left(\mathrm{F}_{(3,12)}=13.56 ; \mathrm{p}<0.0004\right)$. (D) Also as anticipated, Fos mRNA levels peaked in the dark phase in both genotypes. Two-way ANOVA indicated a significant interaction of genotype $\mathrm{x}$ time $\left(\mathrm{F}_{(3,12)}=67.56\right.$; $\mathrm{p}<0.0001$ ). (E) The myelin gene $C n p$ only showed slight differences in regulation over time, with a minimum of expression at ZT22 in both genotypes. (F) Plp, (G) Mbp and (H) Mal indicated a similar pattern of expression in the WT mice with an overall peak at ZT16 (parallel to Per2 and Fos expression), whereas in the mutant mice this degree of regulation was reduced. Cnp: Two-way ANOVA calculated a non significant interaction of genotype and time $\left(\mathrm{F}_{(3,12)}=0.91 ; \mathrm{p}>0.05\right)$, Plp: Two-way ANOVA revealed a highly significant interaction of genotype and time $\left(\mathrm{F}_{(3,12)}=29.48 ; \mathrm{p}<0.0001\right)$, Mal: Two-way ANOVA resulted in a highly significant interaction of genotype and time $\left(\mathrm{F}_{(3,12)}=123.56 ; \mathrm{p}<0.0001\right), M b p$ : Twoway ANOVA showed a highly significant interaction of genotype and time $\left(\mathrm{F}_{(3,12)}=103.40 ; \mathrm{p}<0.0001\right)$. Data for ZT4 and ZT22 were plotted twice (indicated in grey). The lowest value was set to 1 . Data represent mean values $\pm \mathrm{SD}$. Statistical analysis was performed by two-way ANOVA, the p-value depicted in the figures refers to the interaction of genotype and time. White and black bars indicate the light/dark cycle. ACC, anterior cingulate cortex; ZT, zeitgeber time; WT, wild type; S1/2 ${ }^{-/}$, SHARP1 and -2 double null-mutant; SD, standard deviation; ANOVA, analysis of variance. 


\subsubsection{Circadian and activity-dependent regulation of myelin genes in the SSMCX micro-area in WT mice and attenuated effects in $\mathrm{S} 1 / 2^{-/-}$mice}

Another cortical area containing the somatosensory and motor cortices (SSMCx) was collected using LCM. As for the ACC micro-region, qRT-PCR data again were normalised to the housekeeping genes Atp5b and Cycl (Fig. 11A,B), as both showed stable expression profiles along the analysed time points.

Per2 expression displayed a prominent peak in the dark phase at ZT16 for both genotypes (Fig. 11C). Expression slightly increased from ZT4 to ZT10 resulting in a peak at ZT16 (5.3fold for both WT and S1/2/- mice) and further decreased. Two-way ANOVA showed a significant result for the interaction of genotype (WT/S1/2-- $)$ and time $\left(\mathrm{F}_{(3,12)}=50.31\right.$; $\mathrm{p}<0.0001)$.

Consistent with the literature, Fos mRNA was up-regulated in the dark phase (ZT16 and ZT22) in both genotypes, potentially reflecting increased neuronal activity (Fig. 11D). Fos expression revealed the same pattern in $\mathrm{WT}$ and $\mathrm{S} 1 / 2^{-/}$mice. The mRNA concentration declined from ZT4 to the trough at ZT10 and subsequently raised again at ZT16 and ZT22, illustrated by the peak of expression in WT (4.8-fold difference) and in the mutant mice (factor 2), respectively. Fos amplitude appeared to be reduced in the knockout mice. Twoway ANOVA indicated a significant interaction of genotype (WT/S1/2 ${ }^{-/}$) and time $\left(\mathrm{F}_{(3,12)}=66.03 ; \mathrm{p}<0.0001\right)$. Again, Per2 and Fos expression profiles assessed good experimental performance.

Compared to the other myelin genes, $C n p$ mRNA was only moderately regulated in WT mice indicating a mild trough at ZT10 (Fig. 11E). The maximum regulation was 1.6-fold. The expression pattern in mutant mice was inversely to that of control mice, with a moderate peak of expression at ZT10 (1.4-fold change). In summary, Cnp was higher expressed although regulated to a minor extent in mutant mice. Two-way ANOVA determined a very significant interaction of genotype $\left(\mathrm{WT} / \mathrm{S} 1 / 2^{-/-}\right)$and time $\left(\mathrm{F}_{(3,12)}=46.48 ; \mathrm{p}<0.0001\right)$.

In WT mice, Plp mRNA seemed to be co-regulated with Fos, indicating a down-regulation from ZT4 to the trough at ZT10, followed by a 4.5-fold increase at ZT16 (Fig. 11F). In mutant mice, $P l p$ regulation was strongly attenuated (1.2-fold overall change). The expression levels were comparable between genotypes. Two-way ANOVA revealed a highly significant interaction of genotype $\left(\mathrm{WT} / \mathrm{S} 1 / 2^{-/}\right) \times$time $\left(\mathrm{F}_{(3,12)}=122.48 ; \mathrm{p}<0.0001\right)$. 
In WT mice, Mal expression also displayed an IEG-like pattern showing a trough at ZT10 and a 4.4-fold induction at ZT16 (Fig. 11G). At ZT22, mRNA levels were again declining. As already observed for other myelin genes, mutant mice showed a reduced amplitude of Mal expression compared to the WT mice. ZT4 indicated a weak trough of expression, whereas mRNA levels increased in the dark phase (1.4-fold change for both ZT16 and ZT22). Taken together, the level of Mal expression was increased in the mutant mice compared to the WT animals. Two-way ANOVA resulted in a highly significant interaction of genotype (WT/S1/2$\left.{ }^{\prime}\right)$ and time $\left(\mathrm{F}_{(3,12)}=212.60 ; \mathrm{p}<0.0001\right)$.

In the WT mice, $M b p$ regulation differed from the expression profiles of the other myelin genes by showing a prominent peak of expression at ZT10, which represented the trough for Cnp, Plp and Mal (Fig. 11H). For all other time points, Mbp expression only showed minor variability. $M b p$ was induced 6.4-fold from peak to trough. Consistent with other myelin genes, the mutant mice displayed an attenuated $M b p$ regulation. Two-way ANOVA resulted in a highly significant interaction of genotype and time $\left(\mathrm{F}_{(3,12)}=1400.52 ; \mathrm{p}<0.0001\right)$.

As already seen for the ACC micro-area, myelin genes displayed a dynamic day timedependent regulation in WT mice, whereas in the mutant mice these effects were reduced. In WT animals, $P l p$ and $M b p$ very much resembled Per 2 and Fos expression profiles. 
Reference genes

A

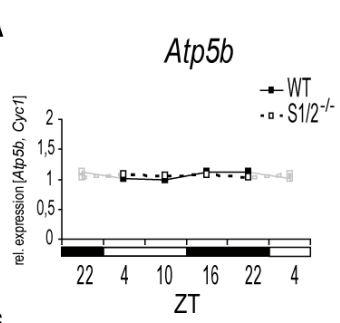

B

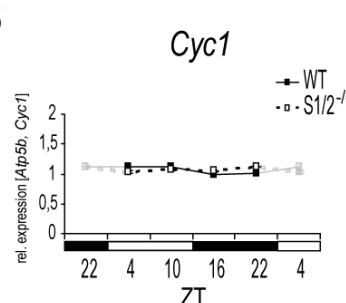

Control genes

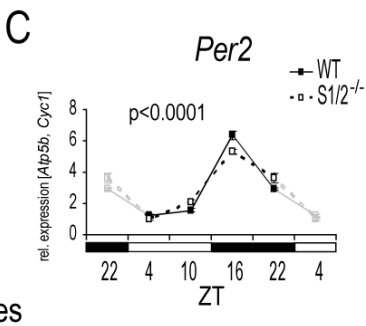

D

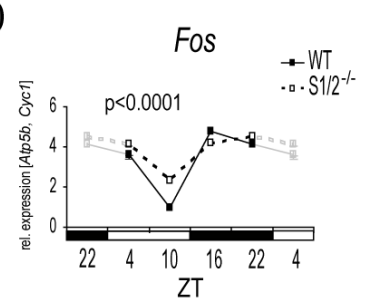

Myelin genes

$E$

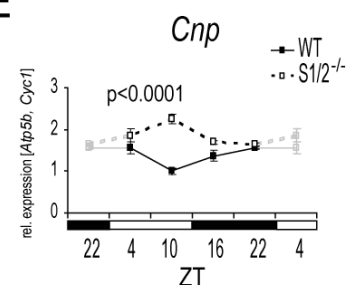

$\mathrm{F}$

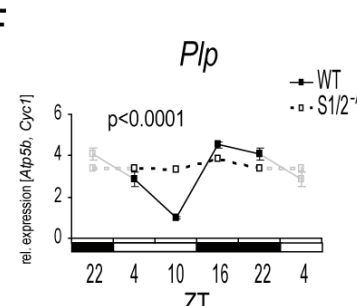

G

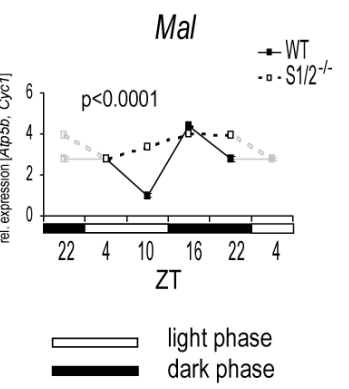

$\mathrm{H}$

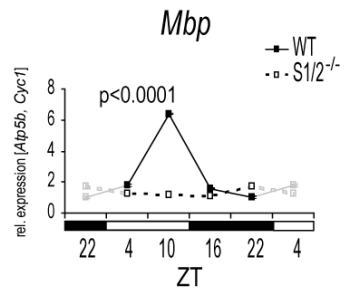

Fig. 11 Circadian and activity-dependent regulation of myelin genes in the SSMCx micro-area in WT mice and attenuated effects in $\mathrm{S} 1 / \mathbf{2}^{-/-}$mice

Gene expression analysis was performed by qRT-PCR. (A,B) Expression levels were normalised to the housekeeping genes Atp5b and Cycl, which both revealed stable profiles. (C) As expected, Per2 expression was up-regulated in both WT and $\mathrm{S} 1 / 2^{-/-}$mice at ZT16, which indicated optimal experimental performance. Two-way ANOVA showed a significant result for the interaction of genotype (WT/S1/2 ${ }^{-/}$) and time $\left(\mathrm{F}_{(3,12)}=50.31 ; \mathrm{p}<0.0001\right)$. (D) Also consistent with the literature, Fos mRNA levels peaked in the dark phase in both genotypes. Two-way ANOVA indicated a significant interaction of genotype and time $\left(\mathrm{F}_{(3,12)}=66.03 ; \mathrm{p}<0.0001\right)$. The myelin genes (E) Cnp, (F) Plp and (F) Mal in WT animals showed a trough at ZT10 and a peak of expression in the dark phase, resembling patterns of Fos and Per2. In mutant animals, this regulation appeared to be attenuated. $(\mathrm{H}) \mathrm{Mbp}$ expression was different, as WT mice indicated a strong peak at ZT10, and mutant mice, however, displayed no obvious variances over time. Cnp: Two-way ANOVA determined a significant interaction of genotype and time $\left(\mathrm{F}_{(3,12)}=46.48\right.$; $\mathrm{p}<0.0001)$; Plp: Two-way ANOVA revealed a highly significant interaction of genotype and time $\left(\mathrm{F}_{(3,12)}=122.48 ; \mathrm{p}<0.0001\right) ;$ Mal: Two-way ANOVA resulted in a highly significant interaction of genotype and time $\left(\mathrm{F}_{(3,12)}=212.60 ; \mathrm{p}<0.0001\right) ; \mathrm{Mbp}$ : Two-way ANOVA showed a highly significant interaction of genotype and time $\left(\mathrm{F}_{(3,12)}=1400.52 ; \mathrm{p}<0.0001\right)$. Data for ZT4 and ZT22 were plotted twice (indicated in grey). The lowest value was set to 1 . Data represent mean values \pm SD. Statistical analysis was performed by two-way ANOVA, the p-value depicted in the figures refers to the interaction of genotype and time. White and black bars indicate the light/dark cycle. SSMCX, somatosensory motor cortex; ZT, zeitgeber time; WT, wild type; $\mathrm{S} 1 / 2^{-/}$, SHARP1 and -2 double null-mutant; SD, standard deviation; ANOVA, analysis of variance. 


\subsubsection{Circadian and activity-dependent regulation of myelin genes in the $\mathrm{CC}$ micro- area in WT mice and attenuated effects in $\mathrm{S} 1 / 2^{-/-}$mice}

qRT-PCR data were normalised to the housekeeping gene Atp $5 b$, which proved to be the most stable reference gene in this micro-area. First, the quality control genes Per2 and Fos were analysed.

Per2 mRNA expression showed a trough at ZT4, increased at ZT10 and finally peaked at ZT16 in both genotypes (7.3-fold induction in WT and 10.8-fold induction in $\mathrm{S} 1 / 2^{-/}$mice, respectively) (Fig. 12A). After ZT16, Per2 mRNA in both genotypes was declining. 2-way ANOVA revealed a significant result for the interaction of genotype (WT/S1/2 ${ }^{-/}$) and time $\left(\mathrm{F}_{(3,12)}=5.35 ; \mathrm{p}=0.0143\right)$. To conclude, Per2 proved good technical performance.

Fos displayed similar kinetic profiles in WT and mutant mice (Fig. 12B). Expression levels declined from ZT4 to ZT10, but then rose again at ZT16, indicating the maximum of expression in WT animals. To note, Fos regulation appeared to be very descent in the CC micro-area (factor 1.7 for WT and factor 1.4 for mutant mice) with respect to both cortical areas. Two-way ANOVA showed a significant result for the interaction of genotype $\left(\mathrm{WT} / \mathrm{S} 1 / 2^{-/-}\right)$and time $\left(\mathrm{F}_{(3,12)}=17.53 ; \mathrm{p}=0.0001\right)$.

In WT mice, the expression level of the myelin gene Cnp slightly increased from ZT4 to ZT10 followed by a down-regulation at ZT16 (factor 2.5), which represented the trough of expression (Fig. 12C). From ZT16 to ZT22 Cnp expression was again enhanced. The mutant mice revealed a mild peak at ZT4, the bottom peak of Cnp also occurred at ZT16, however, the extent of regulation was smaller. The maximum regulation from peak to trough was 1.6fold. Two-way ANOVA displayed a significant interaction of genotype and time $\left(\mathrm{F}_{(3,12)}=62.31 ; \mathrm{p}<0.0001\right)$.

Plp mRNA in WT mice exhibited a similar pattern of regulation as Cnp with a bottom peak at ZT16 (Fig. 12D). The amount of mRNA first slightly rose from ZT4 to ZT10, then went down to the trough of expression at ZT16 and was most abundant at ZT22, which indicated the peak of expression (factor of regulation from peak to trough 2.4). The mutant mice presented an attenuated level of Plp mRNA regulation compared to WT mice. Plp showed no changes in expression during the first three time points but then revealed a trough at ZT22 (factor 1.6 to ZT16). Two-way ANOVA revealed a highly significant interaction of genotype and time $\left(\mathrm{F}_{(3,12)}=70.41 ; \mathrm{p}<0.0001\right)$. 
The WT pattern of Mal expression was reminiscent of the WT Plp regulation whereas the degree was higher (factor 3.6) (Fig. 12E). The mutant mice showed a marginal up-regulation from ZT4 to ZT16 followed by a strong induction with a prominent peak of expression at ZT22 (factor 5.6). Two-way ANOVA resulted in a highly significant interaction of genotype and time $\left(\mathrm{F}_{(3,12)}=608.89 ; \mathrm{p}<0.0001\right)$.

Mbp mRNA also appeared to be less abundant at ZT16 in the WT situation (Fig. 12F). The expression was relatively stable from ZT4 to ZT10, then decreased at ZT16 and peaked at ZT22 (2.1-fold regulation from peak to trough). In S1/2/-- mice, the degree of regulation was again smaller, but the pattern was comparable to that of the WT mice with a minimum at ZT16. The maximum of expression occurred at ZT10 (1.5-fold regulation). Two-way ANOVA resulted in a highly significant interaction of genotype and time $\left(\mathrm{F}_{(3,12)}=46.29\right.$; $\mathrm{p}<0.0001)$.

Of note, the CC micro-area also revealed a dynamic, daytime-dependent regulation of myelin genes in WT animals, whereas the mutant mice again showed diminished amplitudes for most genes (with the exception of $\mathrm{Mal}$ ). However, myelin gene expression in WT animals appeared to be inversely regulated in comparison to the activity marker genes Per2 and Fos, which stayed in contrast to the co-regulation of myelin genes in the cortical micro-areas. 


\section{Control genes}

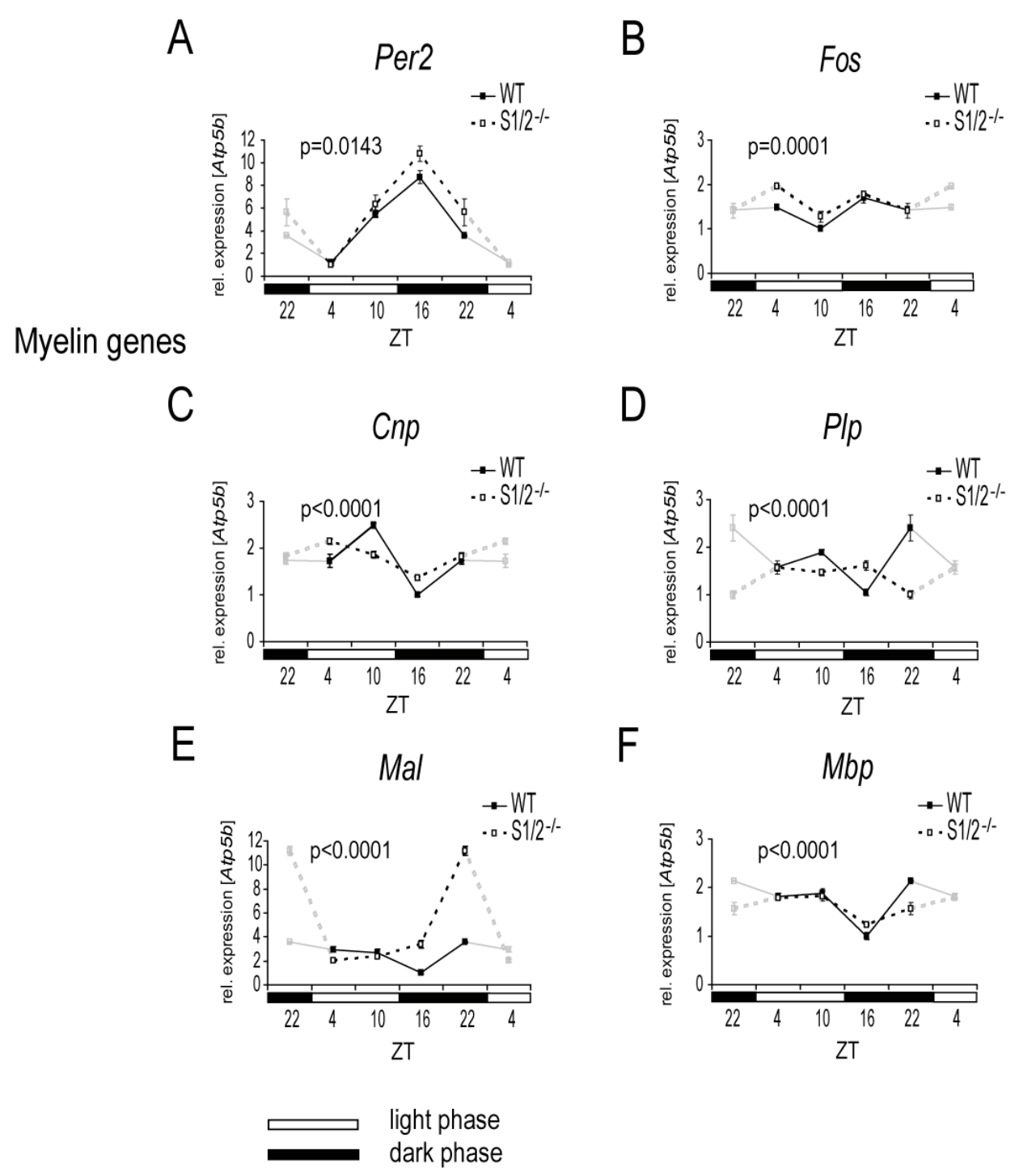

Fig. 12 Circadian and activity-dependent regulation of myelin genes in the $\mathrm{CC}$ micro-area in WT mice and attenuated effects in $\mathrm{S} 1 / 2^{-/-}$mice

Gene expression analysis was performed by qRT-PCR. (A) Per2 expression expectedly was up-regulated in both WT and $\mathrm{S} 1 / 2^{-/}$mice at ZT16, which indicated optimal experimental performance. Two-way ANOVA showed a significant result for the interaction of genotype $\left(\mathrm{WT} / \mathrm{S} 1 / 2^{-/-}\right)$and time $\left(\mathrm{F}_{(3,12)}=5.35\right.$; $\mathrm{p}=0.0143$ ). (B) Fos mRNA only was moderately regulated in both genotypes, indicating a mild peak of expression at ZT16 in WT mice. Two-way ANOVA revealed a significant result for the interaction of genotype $\left(\mathrm{WT} / \mathrm{S} 1 / 2^{-/}\right)$and time $\left(\mathrm{F}_{(3,12)}=17.53 ; \mathrm{p}=0.0001\right)$. The myelin genes (C) Cnp, (D) Plp, (E) Mal and (F) $M b p$ showed a similar pattern of expression in WT animals, with an overall trough at ZT16 (inverse to Per2 expression), whereas in the mutant mice this degree of regulation was reduced, by maintaining a similar pattern (except for $P l p$ ). Another prominent exception was the strong regulation of Mal at ZT22. Cnp: Two-way ANOVA revealed a significant interaction of genotype and time $\left(\mathrm{F}_{(3,12)}=62.31 ; \mathrm{p}<0.0001 ;\right.$ Plp: Two-way ANOVA showed a highly significant interaction of genotype and time $\left(\mathrm{F}_{(3,12)}=70.41 ; \mathrm{p}<0.0001\right) ;$ Mal: Two-way ANOVA resulted in a highly significant interaction of genotype and time $\left(\mathrm{F}_{(3,12)}=608.89 ; \mathrm{p}<0.0001\right) ; M b p$ : Two-way ANOVA displayed a highly significant interaction of genotype and time $\left(\mathrm{F}_{(3,12)}=46.29 ; \mathrm{p}<0.0001\right)$. Data for ZT4 and ZT22 were plotted twice (indicated in grey). The lowest value was set to 1 . Data represent mean values \pm SD. Statistical analysis was performed by two-way ANOVA, the p-value depicted in the figures refers to the interaction of genotype and time. White and black bars indicate the light/dark cycle. CC; corpus callosum; ZT, zeitgeber time; WT, wild type; S1/2 ${ }^{-/}$, SHARP1 and -2 double null-mutant; SD, standard deviation; ANOVA, analysis of variance. 


\subsubsection{Activity-dependent regulation of myelin genes in the $\mathrm{CC}$ micro-area in WT mice}

To validate the findings of the $\mathrm{CC}$ micro-area, the experiment was repeated with an independent, age-matched WT cohort with three biological replicates. qRT-PCR data were again normalised to the housekeeping gene Atp5b. To ensure both optimal technical and experimental performance Per 2 and Fos gene expression levels were again analysed first. In accordance with the literature, Per 2 mRNA expression indicated a peak at ZT16 (Fig. 13A), expression was induced 3-fold from ZT4 to ZT16 and went down again at ZT22.

As already observed for the CC micro-area (see above), Fos regulation was relatively low. mRNA abundance went constantly down from ZT4 to ZT16, which indicated the trough of expression (1.9-fold regulation) (Fig. 13B).

Cnp showed no difference between ZT4 and 10. However, it was repressed about 2-fold at ZT16, thus representing the trough of expression (Fig. 13C). After ZT16, mRNA levels again increased.

Plp displayed a similar expression profile reminiscent of Cnp, with a trough at ZT16, but the overall regulation was weaker (1.5-fold from ZT4 to ZT16) (Fig. 13D).

Mal was further analysed but revealed no variability in expression (Fig. 13E).

Of note, I could validate my findings taken from the CC micro-area with an independent cohort for Cnp and Plp in WT mice, with both genes again displaying expression profiles inverted to that of Per2. Moreover, Fos regulation was again shown to be diminished in the CC micro-area. 


\section{Control genes}

A

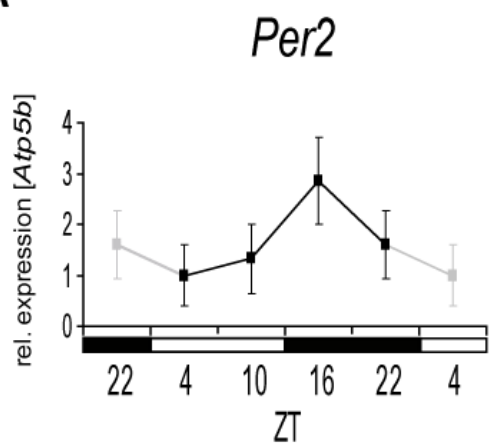

Myelin genes

C

Cnp

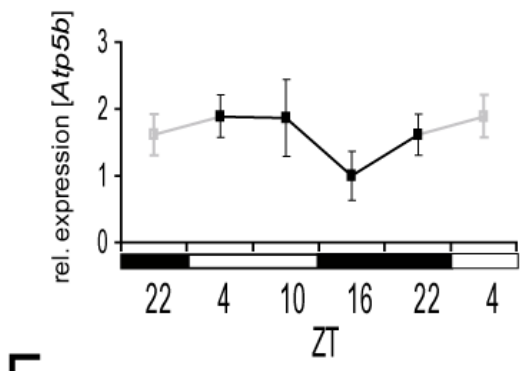

E

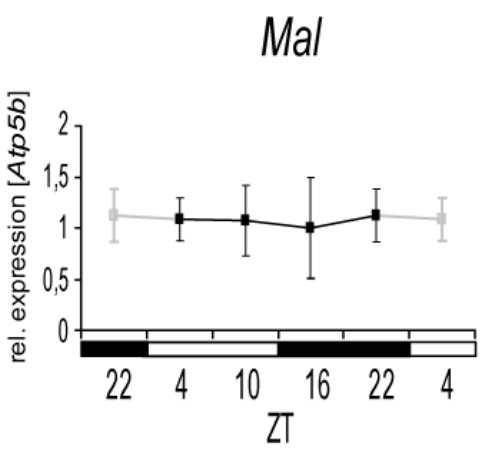

B

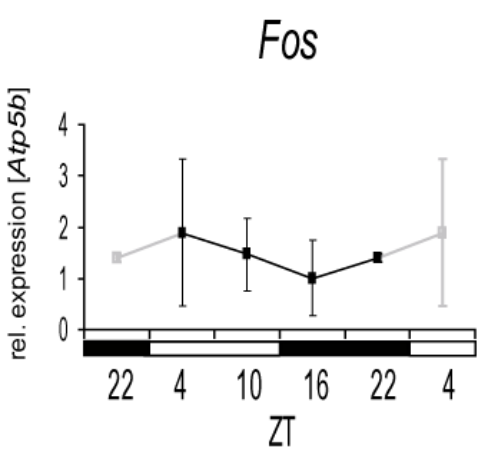

D

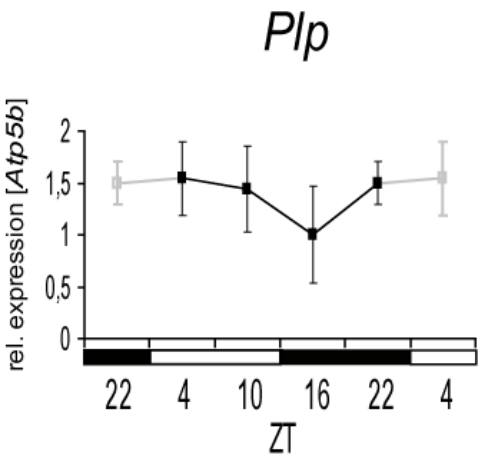

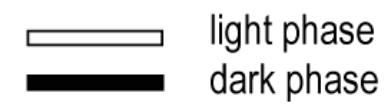

Fig. 13 Circadian and activity-dependent regulation of myelin genes in the CC micro-area in WT mice

Gene expression analysis was performed by qRT-PCR. (A) Per2 served as a quality control gene, showing a peak at ZT16, which is in the middle of the activity phase of mice. (B) Fos only displayed a weak regulation and presented a trough at ZT16. The myelin genes (C) Cnp and (D) Plp were inversely regulated in comparison to Per2 with each a bottom peak at ZT16, whereas (E) Mal appeared to be relatively stable. qRT-PCR data were normalised to the housekeeping gene Atp $5 b$. Data for ZT4 and ZT22 are plotted twice (indicated in grey). The lowest value is set to 1 . Data represent mean values $\pm \mathrm{SD}$. White and black bars indicate the light/dark cycle. CC; corpus callosum; ZT, zeitgeber time; WT, wild type; S1/2/- , SHARP-1 and -2 double-null mutant; SD, standard deviation. 


\subsection{Attenuated regulation of myelin genes in the sciatic nerve and optic nerve in $\mathrm{WT}$ and $\mathrm{S} 1 / 2^{-/-}$mice}

Next, an experiment was performed in which myelin gene expression in both CNS and PNS nerves was analysed. A cohort of adult (four months of age) WT and $\mathrm{S} 1 / 2^{-/-}$mice was entrained to a 12:12 hour light/dark (LD) cycle and exposed to an enriched environment for two weeks (compare to Fig. 8). Consistent with the experiments before, animals were sacrificed at four different time points ZT4, 10, 16 and 22 with $\mathrm{n}=3$ mice per time point and genotype. For further analysis, corresponding animals were pooled.

\subsubsection{Sciatic nerve}

Gene expression was normalised to the housekeeping genes $\beta$-Actin and Atp $5 b$, as both genes showed the most stable expression profiles among several reference genes tested (Fig. $14 \mathrm{~A}, \mathrm{~B})$.

Per2 expression again was taken as a technical control and displayed a strong up-regulation from ZT4 to ZT10 (8.7-fold in the WT and 4.3-fold in mutant mice) (Fig. 14C). The levels remained elevated at ZT16 showed a decline at ZT22. The expression profile was very similar between WT and mutant mice; however, the level was overall higher in the mutants. Twoway ANOVA showed a very significant result for the interaction of genotype (WT/S1/2 ${ }^{-/}$) and time $\left(\mathrm{F}_{(3,12)}=27.21 ; \mathrm{p}<0.0001\right)$.

Fos regulation was relatively weak compared to the cortical micro-areas (Fig. 14D). Nevertheless, WT mice revealed an elevated expression in the darkness (ZT16 and ZT22) (1.2-fold regulation), potentially consistent with increased (neuronal) activity. The Fos kinetics appeared to be a bit earlier in the knockout mice, an up-regulation could already been observed at ZT10 and was further enhanced to the peak at ZT16 (factor 1.4). Fos showed similar mRNA abundance among the groups. Two-way ANOVA indicated a significant result for the interaction of genotype $\left(\mathrm{WT} / \mathrm{S} 1 / 2^{-/}\right)$and time $\left(\mathrm{F}_{(3,12)}=30.38 ; \mathrm{p}<0.0001\right)$.

Cnp hardly showed any day time-dependent variability in expression with comparable expression levels between genotypes, except for ZT4 (Fig. 14E). A small peak could be observed at ZT16 for both wild type (1.2-fold) and mutant mice (1.4-fold). Two-way ANOVA revealed a highly significant interaction of genotype and time $\left(\mathrm{F}_{(3,12)}=18.35\right.$; $\mathrm{p}<0.0001)$. 
Plp was regulated in a similar manner to $C n p$, as it showed the same kinetics for both genotypes (Fig. 14F). Plp expression only displayed minor differences, with a mild peak at ZT16 in control (1.2-fold) and mutant (1.4-fold) mice. Of note, both kinetics looked identical, with the only difference that $P l p$ was lower expressed in the mutant mice. Two-way ANOVA resulted in a non significant interaction of genotype and time $\left(F_{(3,12)}=0.07 ; p=0.9750\right)$.

Mal again showed the strongest regulation among myelin genes in both genotypes (Fig. 14G). Expression increased from the trough at ZT4 to the peak at ZT10 in both animal groups (factor 2.6 for WT and 1.6 in the mutant mice) and then further declined. The level of Mal appeared to be slightly elevated in mutant mice. Two-way ANOVA indicated a highly significant interaction of genotype and time $\left(\mathrm{F}_{(3,12)}=11.62 ; \mathrm{p}=0.0007\right)$.

Taken together, the sciatic nerve only displayed a weak dynamic regulation of myelin genes as exemplified for Cnp and Plp. However, a tendency for a peak at ZT16 could be shown. No substantial differences among genotypes were detected. The regulation of $\mathrm{Mal}$ appeared to be different from the other myelin genes, as it was substantially induced from ZT4 to ZT10. Also, Fos regulation was weak. 
Reference genes

A

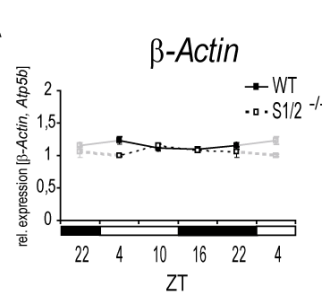

B

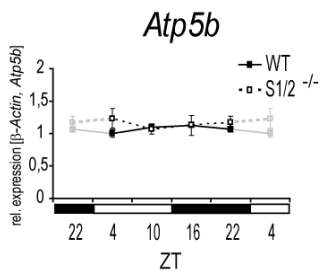

Control genes

C

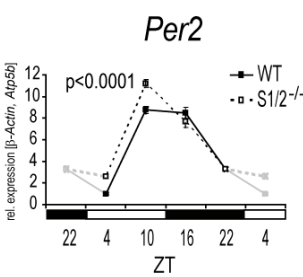

D

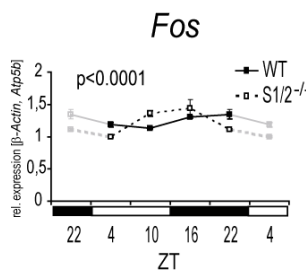

Myelin genes

$E$

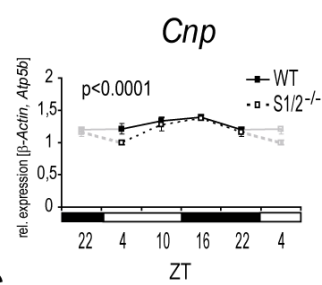

G

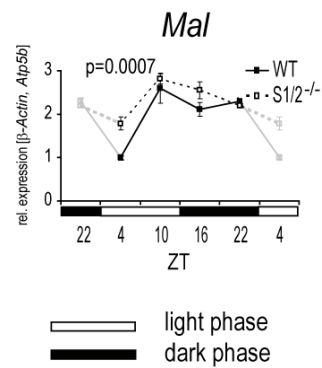

$\mathrm{F}$

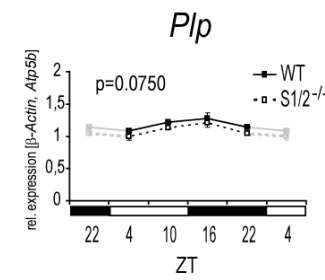

Adult (four months of age), male mice were kept in 12:12 LD rhythm, exposed to an enriched environment for two weeks before animals were sacrificed at four different time points: ZT4, 6, 10 and 22 (Fig.9). Sciatic nerves were dissected and immediately frozen for qRT-PCR analysis. (A,B) qRT-PCR data were normalised to the housekeeping genes $\beta$-Actin and Atp5b. (C) Per2 expression analysis indicated good technical performance by showing high expression levels in the dark phase at ZT16 in WT and mutant mice. Two-way ANOVA revealed a very significant result for the interaction of genotype $\left(\mathrm{WT} / \mathrm{S} 1 / 2^{-/}\right)$and time $\left(\mathrm{F}_{(3,12)}=27.21 ; \mathrm{p}<0.0001\right)$. (D) Fos regulation was relatively mild in both groups, showing elevated expression during the dark phase for WT animals and earlier kinetics for mutant mice. Two-way ANOVA calculated a significant result for the interaction of genotype (WT/S1/2 $\left.{ }^{-/}\right)$and time $\left(\mathrm{F}_{(3,12)}=30.38 ; \mathrm{p}<0.0001\right)$. (E) $C n p$ and $(\mathrm{F})$ Plp expression analysis indicated a similar regulation of these genes with minor differences between genotypes. A mild increase of mRNA abundance could be observed for both genes and groups at ZT16. Cnp: Two-way ANOVA showed a highly significant interaction of genotype and time $\left(\mathrm{F}_{(3,12)}=18.35 ; \mathrm{p}<0.0001\right)$; Plp: Two-way ANOVA resulted in a non significant interaction of genotype and time $\left(\mathrm{F}_{(3,12)}=0.07 ; \mathrm{p}=0.9750\right)$. (G) Mal regulation was shown to be the strongest among myelin genes with a similar pattern in both genotypes. Mal mRNA was up-regulated from ZT4 to the trough of expression at ZT16 and remained elevated during the dark time points. Twoway ANOVA indicated a highly significant interaction of genotype and time $\left(\mathrm{F}_{(3,12)}=11.62 ; \mathrm{p}=0.0007\right)$. Data for ZT4 and ZT22 were plotted twice (indicated in grey). The lowest value was set to 1 . Data represent mean values $\pm \mathrm{SD}$. Statistical analysis was performed by two-way ANOVA, the p-value depicted in the figures refers to the interaction of genotype and time. White and black bars indicate the light/dark cycle, ZT, zeitgeber time; WT, wild type; $\mathrm{S} 1 / 2^{-/}$, SHARP-1 and -2 double null-mutant; LD, light/dark; SD, standard deviation; ANOVA, analysis of variance. 


\subsubsection{Optic nerve}

To this end, I observed that myelin genes are coupled, at least to some extent, to the circadian system and/or to neuronal activity (e.g. the behavioural state). To further elucidate the mechanism that regulates myelin gene expression a CNS nerve, the optic nerve, was analysed. Again, light was used to entrain the animals in a 12:12 hour LD cycle and the mice were sacrificed at ZT4, 10, 16 and 22. In a follow-up approach, animals were also entrained to an LD cycle and released into constant darkness (DD). On the first day of DD, the mice were sacrificed at four different time points, circadian time (CT) 4, 10, 16 and 22. The hypothesis was that light input in the LD cycle would result in an increased neuronal activity in the optic nerve, which in turn might influence myelin gene expression, an effect that could potentially differ from DD conditions.

The LD experiment is depicted on the left side, the corresponding data of the DD analysis are shown on the right side. qRT-PCR data were normalised to the housekeeping genes $\beta$-Actin and $A t p 5 b$, as these genes revealed the most robust activity-dependent expression profile (Fig. 15A-D).

The circadian marker gene Per2 displayed a peak of expression in the dark phase at ZT16 in both genotypes and both experiments (Fig. 15E,F). In LD, the expression levels and patterns of regulation were very similar between WT and mutant mice. In detail, the expression was enhanced from ZT4 to ZT10 by 2.6-fold in the WT and by 2.3 -fold in S1/2 ${ }^{-/}$mice. At ZT16, there was even a mild increase detectable. At ZT22, both genotypes displayed a reduction of Per2 mRNA expression. Two-way ANOVA showed a significant result for the interaction of genotype $\left(\mathrm{WT} / \mathrm{S} 1 / 2^{-/-}\right)$and time $\left(\mathrm{F}_{(3,12)}=10.10 ; \mathrm{p}=0.0013\right)$.

In constant darkness (DD), the regulation of Per2 in WT animals was very similar to that in LD, with a strong induction from ZT4 to ZT10 (Fig. 15F). Mutant mice displayed a comparable pattern, although the mRNA abundance increased slower from ZT4 to the peak at ZT16 (2.4-fold). Two-way ANOVA indicated a significant interaction of genotype and time $\left(\mathrm{F}_{(3,12)}=7.11 ; \mathrm{p}=0.0053\right)$. Under both conditions, Per 2 expression levels were similar between control and mutant mice and consistent with published data.

In LD, Fos mRNA abundance in WT mice peaked at ZT4, was then subsequently repressed at ZT10 by 1.7-fold, and slowly raised again (Fig. 15G). Mutant mice showed a similar pattern of expression as Fos was down-regulated from ZT4 to the trough at ZT10 followed by an 1.5fold induction to the maximal expression at ZT22. Fos levels were higher in mutant mice for 
all time points monitored. Two-way ANOVA showed a significant interaction of genotype and time $\left(\mathrm{F}_{(3,12)}=50.63 ; \mathrm{p}<0.0001\right)$.

Under DD conditions, Fos regulation in WT mice displayed only minimal variability (Fig. $15 \mathrm{H})$. In S1/2/- mice, Fos mRNA abundance constantly increased 1.6-fold from a minimal expression at ZT4 to the maximum at ZT22. Two-way ANOVA revealed a significant interaction of genotype and time $\left(\mathrm{F}_{(3,12)}=75.55 ; \mathrm{p}<0.0001\right)$.

Cnp mRNA showed moderate cycling behaviour in WT and S1/2 ${ }^{-/-}$mice in LD (Fig. 15I). Expression increased from ZT4 to a peak at ZT10 and then slowly decreased to the trough at ZT122 (1.3-fold change in both genotypes). Cnp mRNA abundance was lower in the mutant mice for all time points, but revealed the same pattern of regulation by paralleling the WT kinetics. Two-way ANOVA determined a non significant interaction of genotype and time $\left(\mathrm{F}_{(3,12)}=0.15 ; \mathrm{p}=0.9291\right)$.

Cnp WT regulation in DD very much resembled the pattern observed in LD, with a mild peak at ZT10 followed by a trough at ZT22 (a mild 1.2-fold change) (Fig. 15J). For the mutant mice, the kinetics seemed to be shifted forwards for four hours, with a peak already at ZT4 and a trough at ZT16 (factor 1.3). The relative mRNA abundance was comparable between WT and mutant mice. Two-way ANOVA revealed a very significant interaction of genotype and time $\left(\mathrm{F}_{(3,12)}=7.95 ; \mathrm{p}=0.0035\right)$.

Plp gene expression under LD and DD conditions appeared to be relatively stable with minor variations in WT and mutant mice (Fig. 15K,L). However, the amount of Plp mRNA was lower in mutant mice for all time points taken. Two-way ANOVA for the LD experiment resulted in a significant interaction of genotype and time $\left(\mathrm{F}_{(3,12)}=6.47 ; \mathrm{p}=0.0075\right)$. Two-way ANOVA for the DD analysis displayed a significant interaction of genotype and time $\left(\mathrm{F}_{(3,12)}=6.57 ; \mathrm{p}=0.0071\right)$.

Mal, again as the only analysed myelin gene, showed at least in the WT mice a prominent regulation with a peak at ZT4 (Fig. 15M,N). Later, its expression declined to reach the trough at ZT16 (DD condition, 1.6-fold change) and at ZT22 (LD cycle, 1.8-fold change). The mutant mice kept in the LD rhythm revealed only slight differences in Mal regulation, whereas in DD conditions the expression profile was similar between genotypes. Two-way ANOVA for the LD experiment indicated a highly significant interaction of genotype and time $\left(\mathrm{F}_{(3,12)}=46.92 ; \mathrm{p}<0.0001\right)$. Two-way ANOVA for the DD analysis resulted in a non significant interaction of genotype $\left(\mathrm{WT} / \mathrm{S} 1 / 2^{-/-}\right)$and time $\left(\mathrm{F}_{(3,12)}=1.03 ; \mathrm{p}=0.4145\right)$. 
Taken together, expression analysis of myelin genes in the optic nerve only showed mild regulation (except for $M a l$ ), with minor differences between LD and DD experiment and between genotypes. Of note, $C n p$ and Plp often appeared to be co-regulated (see other microareas and sciatic nerve), with small differences in time (sciatic and optic nerve), whereas the expression of Mal showed strong differences both in amplitude and pattern (sciatic and optic nerve). 


\section{LD cycle DD cycle}

Reference genes
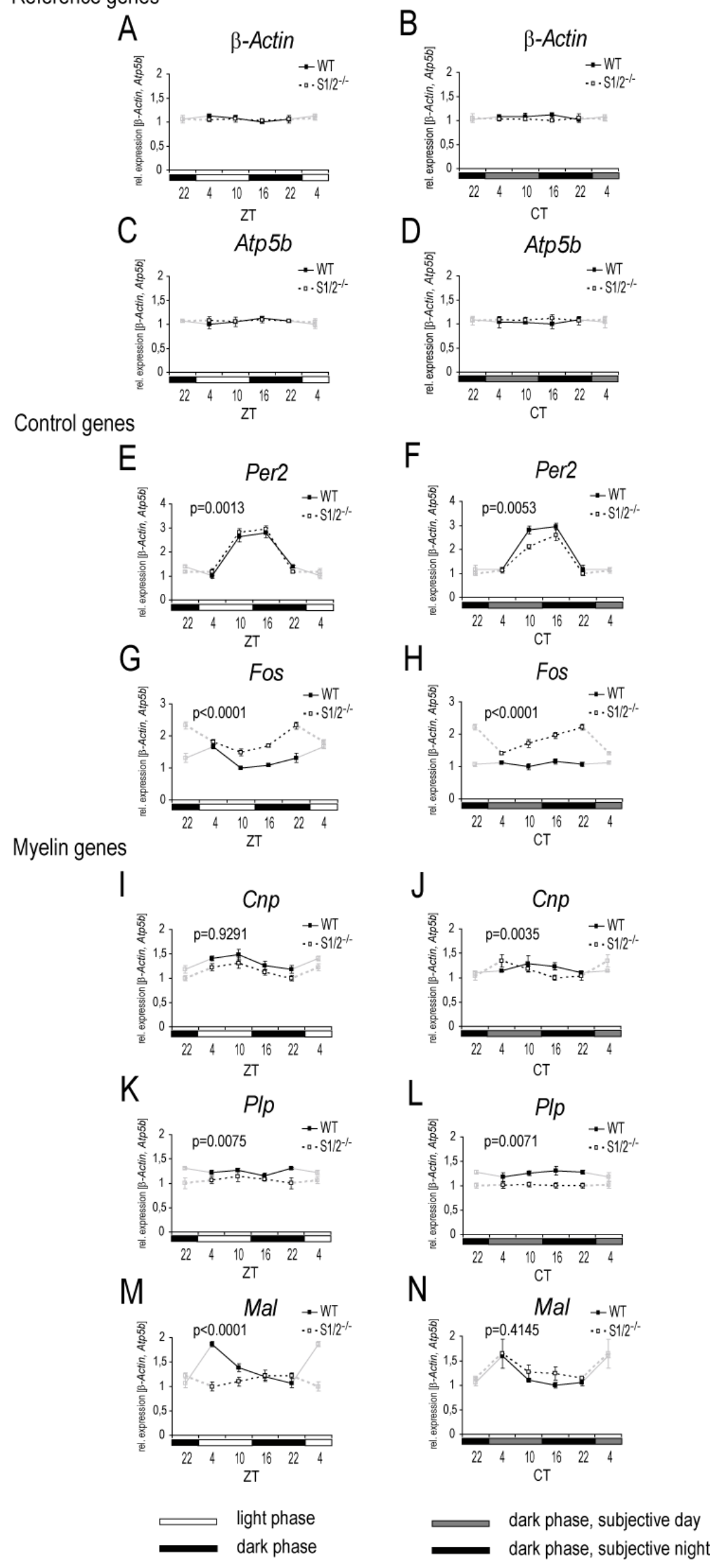

F 
Fig. 15 Attenuated regulation of myelin genes in the optic nerve in WT and $\mathrm{S} 1 / 2^{-/-}$mice under LD and DD conditions

To differentiate between effects of the behavioural state and the circadian system, myelin gene expression was analysed in the optic nerve under two conditions. In the first experiment, animals were kept in a normal LD rhythm, whereas for the second analysis, mice were entrained to an LD cycle and released into constant darkness (DD). Mice were sacrificed at ZT 4, 6, 10 and 22 (LD cycle) as well as CT4, 6, 10 and 22 (DD rhythm). qRT-PCR data for the LD experiment are depicted on the left side (A,C,E,G,I,K,M), whereas the DD experiment is shown on the right side (B,D,F,H,J,L,N). (A-D) Data were normalised to the housekeeping genes $\beta$-Actin and Atp $5 b$. (E,F) Per2 expression analysis indicated good technical performance by showing the maximum of expression in the dark phase at ZT/CT16 in both experiments and genotypes. Two-way ANOVA indicated a significant result for the interaction of genotype (WT/S1/2-

l-) and time $\left(\mathrm{F}_{(3,12)}=10.10 ; \mathrm{p}=0.0013\right)$ for $\mathrm{LD}$ and $\left(\mathrm{F}_{(3,12)}=7.11 ; \mathrm{p}=0.0053\right)$ for $\mathrm{DD}$. (G) In LD, Fos expression in WT animals went down from ZT4 to ZT10, and slightly increased in the dark phase, whereas in mutant mice, the peak of expression correlated with ZT22. Two-way ANOVA showed a significant interaction of genotype and time $\left(\mathrm{F}_{(3,12)}=50.63 ; \mathrm{p}<0.0001\right)$. (H) In DD, WT animals displayed no variances in expression. $\mathrm{S} 1 / 2^{-/}$mice indicated a peak of expression at $\mathrm{CT} 16$, resembling the LD regulation. Two-way ANOVA revealed a significant interaction of genotype and time $\left(\mathrm{F}_{(3,12)}=75.55\right.$; $\mathrm{p}<0.0001)$. The myelin genes (I,J) Cnp and (K,L) Plp showed only a very mild dynamic regulation with no obvious differences among LD/DD conditions and genotypes, except the lower expression levels in mutant mice. Cnp: Two-way ANOVA determined a non significant interaction of genotype and time $\left(\mathrm{F}_{(3,12)}=0.15 ; \mathrm{p}=0.9291\right)$ for $\mathrm{LD}$ and a significant interaction of genotype and time $\left(\mathrm{F}_{(3,12)}=7.95 ; \mathrm{p}=0.0035\right)$ for DD; Plp: Two-way ANOVA for the LD experiment resulted in a significant interaction of genotype and time $\left(\mathrm{F}_{(3,12)}=6.47 ; \mathrm{p}=0.0075\right)$. Two-way ANOVA for the DD analysis displayed a significant interaction of genotype and time $\left.\left(\mathrm{F}_{(3,12}\right)=6.57 ; \mathrm{p}=0.0071\right)$. $(\mathrm{M}, \mathrm{N})$ The regulation of Mal was stronger (except for mutant mice in LD), indicating a prominent peak at ZT4 (WT mice) and CT4 (WT and S1/2/animals). Two-way ANOVA for the LD experiment indicated a highly significant interaction of genotype and time $\left(\mathrm{F}_{(3,12)}=46.92 ; \mathrm{p}<0.0001\right)$. Two-way ANOVA for the DD analysis resulted in a non significant interaction of genotype $\left(\mathrm{WT} / \mathrm{S} 1 / 2^{-/}\right)$and time $\left(\mathrm{F}_{(3,12)}=1.03\right.$; $\left.\mathrm{p}=0.4145\right)$. Data for ZT/CT4 and ZT/CT22 were plotted twice (indicated in grey). The lowest value was set to 1 . Data represent mean values \pm SD. Statistical analysis was performed by two-way ANOVA, the p-value depicted in the figures refers to the interaction of genotype and time. White and black bars indicate the LD (light/dark) cycle, grey and black bars refer to DD (dark/dark) cycle ZT, zeitgeber time; CT, circadian time; WT, wild type; $\mathrm{S} 1 / 2^{-/}$, SHARP1 and -2 double null-mutant; SD, standard deviation; ANOVA, analysis of variance.

\subsection{Circadian stability of myelin protein levels in the prefrontal cortex and corpus callosum in WT and $\mathrm{S} 1 / 2^{-/-}$mice}

Western blots were performed to test whether the dynamic regulation of myelin gene expression is accompanied by changes in protein levels. Therefore, a new cohort of WT and $\mathrm{S} 1 / 2^{-/-}$mice was analysed. Adult male mice (four months of age) were entrained to a $12 / 12$ LD cycle and kept in an enriched environment for two weeks (compare to Fig. 8). To obtain a high temporal resolution, animals were analysed at six different time points, ZT0, 4, 8, 12, 16 and 20. To compare the myelin mRNA expression profiles of the micro-areas to changes in protein levels the brain slicer was used to precisely isolate the anterior cortex and corpus callosum between Bregma -0.82 and 1.18 mm (Fig. 16A,B). 


\subsubsection{Prefrontal cortex}

CNP1 revealed no explicit kinetic differences among the different time points (Fig. 16C). Also, no obvious alterations in $\alpha$-Tubulin, which was used for normalising protein expression, were detected for WT and S1/2 ${ }^{-/}$, indicating an equal amount of protein loading.

\subsubsection{Corpus callosum}

Western blots prepared from corpus callosum samples were probed with a set of antibodies against myelin surrogate marker proteins, such as CNP, MBP, MAL and PLP (Fig. 16D). No obvious time-dependent differences in myelin protein expression could be observed. $\alpha$ Tubulin indicated equal protein loading.

To this end, I can conclude that there are no obvious circadian differences in protein levels in WT and $\mathrm{S} 1 / 2^{-/-}$mice for both tissues, at least not for the set of investigated myelin proteins. 
A

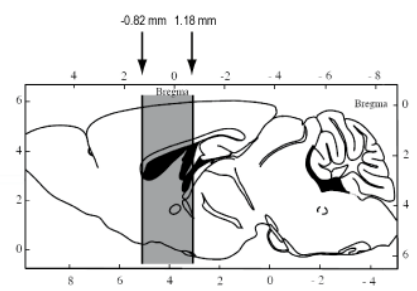

B

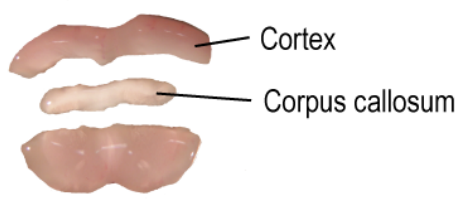

C

\section{Cortex}

WT
$\mathrm{S} 1 / 2^{-1}$

$\begin{array}{llllll}0 & 4 & 8 & 12 & 16 & 20\end{array}$

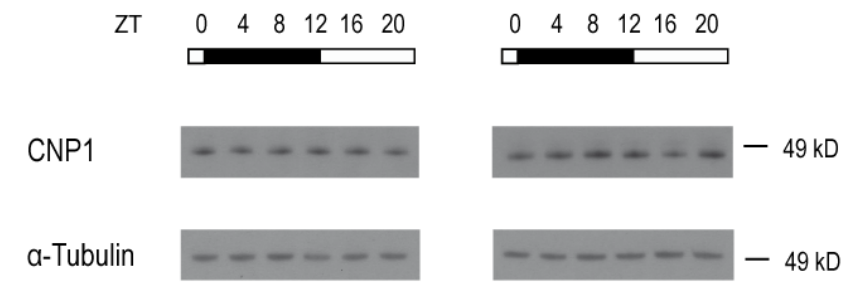

D

\section{Corpus callosum}

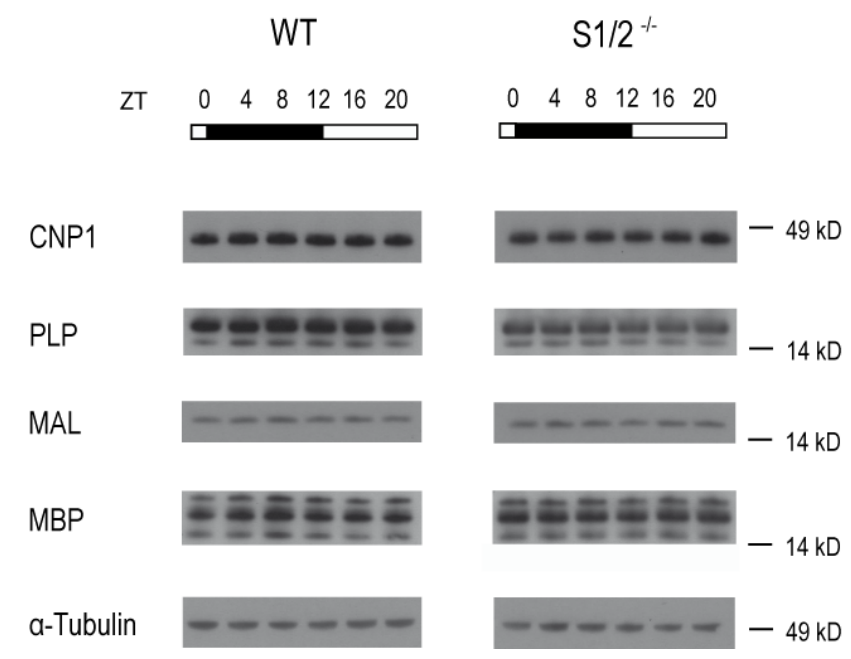

Fig. 16 Circadian stability of myelin protein levels in the prefrontal cortex and corpus callosum in WT and $S 1 / 2^{-/-}$mice

Adult (four months of age), male WT and S1/2 ${ }^{-/}$mice were entrained to a 12:12 LD cycle and exposed to an enriched environment for two weeks. Animals were sacrificed at ZT0, 4, 8, 12, 16 and 20 with $n=3$ mice per time point. (A) The cortex and the corpus callosum were collected at Bregma $-0.82-1.18 \mathrm{~mm}$ using a brain slicer (B). (C) Western blots of cortex samples were probed with an antibody against CNP (46 kD). (D) Western blots of corpus callosum samples were analysed with antibodies against CNP (46 $\mathrm{kD})$, PLP $(25 \mathrm{kD})$, MAL (15 kD), and MBP (18.5 kD). $\alpha$-Tubulin $(50 \mathrm{kD})$ is shown as an internal loading control. The anti-CNP antibody recognized CNP1 which constitutes a protein of $46 \mathrm{kD}$. The anti-MBP antibody identified four different splice variants between 18-20 kD. The anti-PLP antibody showed PLP and its splice variant DM20 (25 and $20 \mathrm{kD}$, respectively). Corresponding animals per time point were pooled. $5 \mu \mathrm{g}$ total protein lysate was loaded. WT, wild type, S1/2/-- SHARP1 and -2 null-mutant; ZT, zeitgeber time; $\mathrm{kD}$, kilo Dalton. 


\subsection{Differential regulation of myelin gene expression upon sleep deprivation in WT and $\mathrm{S} 1 / 2^{-/-}$mice}

To assess the effects of sleep deprivation (SD) on myelin gene expression a cohort of adult male WT and mutant mice ( $\mathrm{n}=6$ per genotype and condition) was continuously sleep deprived for six hours at the beginning of the light ('sleep') phase when sleep pressure is known to be highest (ZT0-ZT6) (Fig. 17A). Therefore, the inverted flowerpot technique (Mendelson et al., 1974) was applied which is known to suppress REM sleep and to reduce NREM sleep (Fig. 17B). Immediately after six hours of SD the animals were sacrificed and the brains were rapidly processed using a brain slicer. The cerebral cortex was collected for analysis because of its fundamental role in generating sleep rhythms and its specific response upon sleep deprivation (Borbély and Achermann, 1999; Van Dongen et al., 2003; Horne, 1988; Steriade and Hobson, 1976).

qRT-PCR data were normalised to the housekeeping genes $\beta$-Actin and Atp5b, which indicated relatively stable expression (Fig. 17C,D). Per2, a core clock gene, which is known to be influenced by both the circadian system as well as by activity (Cirelli et al., 2004) revealed to be significantly down-regulated by factor 3.7 upon SD in WT mice $(\mathrm{p}<0.05)$ (Fig. 17E). In untreated mutant mice Per 2 expression was low in comparison to untreated control mice (factor 2.5) and displayed no difference in expression upon SD in S1/2/- mice.

Fos, an IEG and marker for neuronal activity was down-regulated in response to SD in both WT and S1/2/- mice (3.5-fold and 1.6-fold, respectively) (Fig. 17F), whereas the effect in WT mice was significant, with $\mathrm{p}<0.01$. Like Per 2 , Fos expression was lower in undisturbed mutant mice with respect to undisturbed control mice.

Next, myelin gene expression was analysed. The level of Cnp expression decreased in WT mice upon SD by factor 1.5, whereas in the mutant mice this effect appeared to be strongly attenuated (factor 1.1) (Fig. 17G). In the untreated S1/2/-- group Cnp transcripts displayed less abundance compared to the untreated WT mice.

For Plp, an induction of gene expression upon treatment could be observed in WT mice by factor 1.3, whereas in the mutant mice the opposite case was true, Plp mRNA levels went down upon SD by the same factor of regulation (Fig. 17H).

In WT mice, Mal expression was slightly enhanced in the SD group by factor 1.1, however, in the mutant mice, Mal mRNA expression was lower in the sleep-deprived animals compared to the untreated group (1.3-fold reduction) (Fig. 17I). This effect was significant with $\mathrm{p}<0.01$. 
Mal transcripts showed similar abundance among treated and non-treated WT and treated $\mathrm{S} 1 / 2^{-/}$mice, the level in SD mutant animals appeared to be elevated. Mal regulation very much resembled that of Plp.

The analysis of $M b p$ regulation upon SD was reminiscent to that of Cnp (Fig. 17J). In WT mice, $M b p$ was repressed in response to SD by factor 1.3 , with $\mathrm{p}<0.01$. In mutant mice, this effect was strongly reduced, no obvious differences could be observed.

To conclude, in WT mice, $C n p$ and $M b p$ expression were markedly reduced upon SD, whereas these effects were attenuated in the mutant mice. The regulation for Plp and Mal was different, as in WT mice both genes were induced upon loss of sleep, whereas the opposite was true for mutant mice. Apparently, myelin genes respond differently to the SD paradigm. 
A

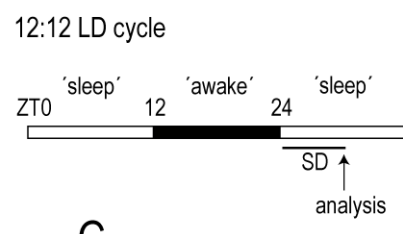

B

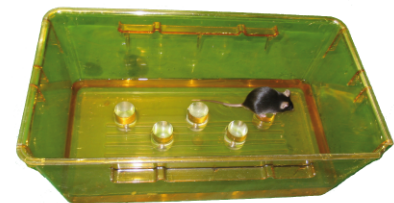

C

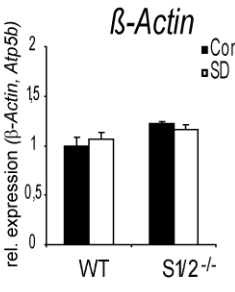

E

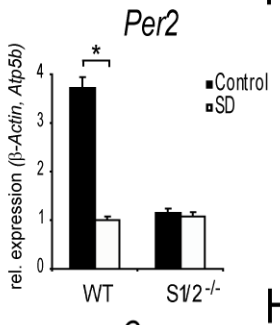

$\mathrm{F}$

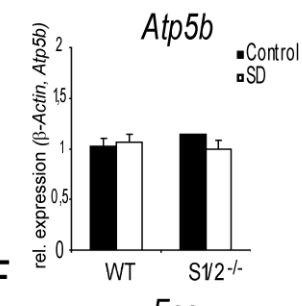

G

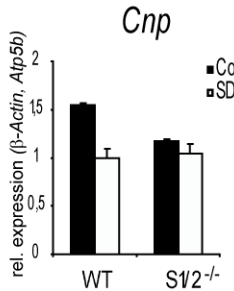

$\mathrm{H}$

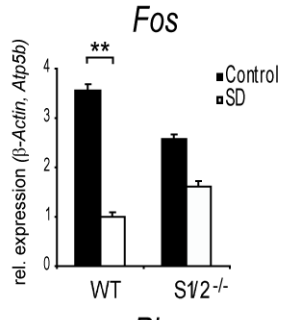

SD

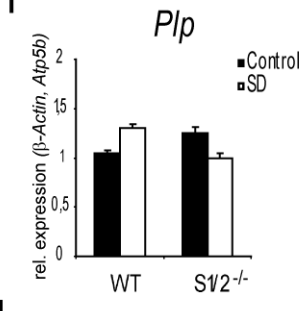

$\mathrm{J}$

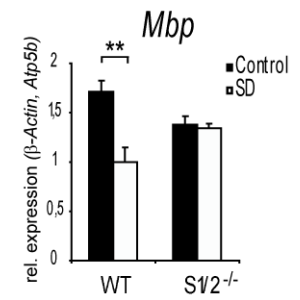

Fig. 17 Differential regulation of myelin gene expression upon sleep deprivation in WT and S1/2 mice

Male WT and $\mathrm{S} 1 / 2^{-/-}$mice at the age of four months ( $\mathrm{n}=6$ per genotype and condition) were entrained to a 12:12 hour light/dark cycle for two weeks. (A) Animals were sleep deprived for six hours from ZT0-ZT6 subsequent to their activity phase using the inverted flower pot technique. (B) Each cage contained five cylinders (inverted flower pots) surrounded by water. Mice were placed in the cages (one mouse per cage) for six hours. Immediately after SD mice were sacrificed and the cortex between Bregma -0.82 and 1.18 mm was dissected using the brain slicer (compare with Fig.17B). Gene expression was analysed by qRTPCR. (C,D) Expression levels were normalized to the housekeeping genes $\beta$-Actin and Atp5b. (E) Per 2 expression was down-regulated upon SD in WT mice $(\mathrm{p}<0.05)$ whereas in mutant animals this response appeared to be strongly attenuated. (F) Fos expression was very similar to the Per2 regulation in WT mice, showing a reduction of expression upon $\mathrm{SD}(\mathrm{p}<0.01)$. In mutant mice this decline could also be observed, but to a smaller extent. (G) Cnp gene expression was down-regulated upon SD in WT mice, whereas this effect was strongly attenuated in mutant mice. (H) The $P l p$ response was characterised by an enhanced expression upon SD in the WT mice and a reduced expression upon SD in the mutant mice. (I) In WT mice, Mal mRNA levels displayed only minor variances in response to SD, but mutant mice exhibited a significant reduction upon SD with $\mathrm{p}<0.01$. (J) $M b p$ appeared to be co-regulated with $C n p$ indicating a reduction after SD in WT mice and almost no differences in mutant mice upon the treatment. The lowest value was set to 1 . Data represent mean values \pm SD. Statistical analysis were performed by the Kruskal Wallis test and Dunn's multiple comparison test. WT, wild type; S1/2-/, SHARP1 and -2 double null-mutant; SD, sleep deprivation; ZT, zeitgeber time. 


\subsection{Dynamic expression of myelin genes in stimulated primary oligodendrocyte cultures and reduced expression levels in $\mathrm{S} 1 / 2^{-/-}$ cultures}

Primary cultured oligodendrocytes were taken as an in vitro model to further study the dynamic regulation of myelin genes and potential differences in $\mathrm{S} 1 / 2^{-/-}$mice. Cultures of differentiated, MBP-positive oligodendrocytes were stimulated with neuronal conditioned medium (NCM) for one hour to mimic neuronal input (Fig. 18).

\subsubsection{Kinetic 1}

\section{pOL Kinetic I}

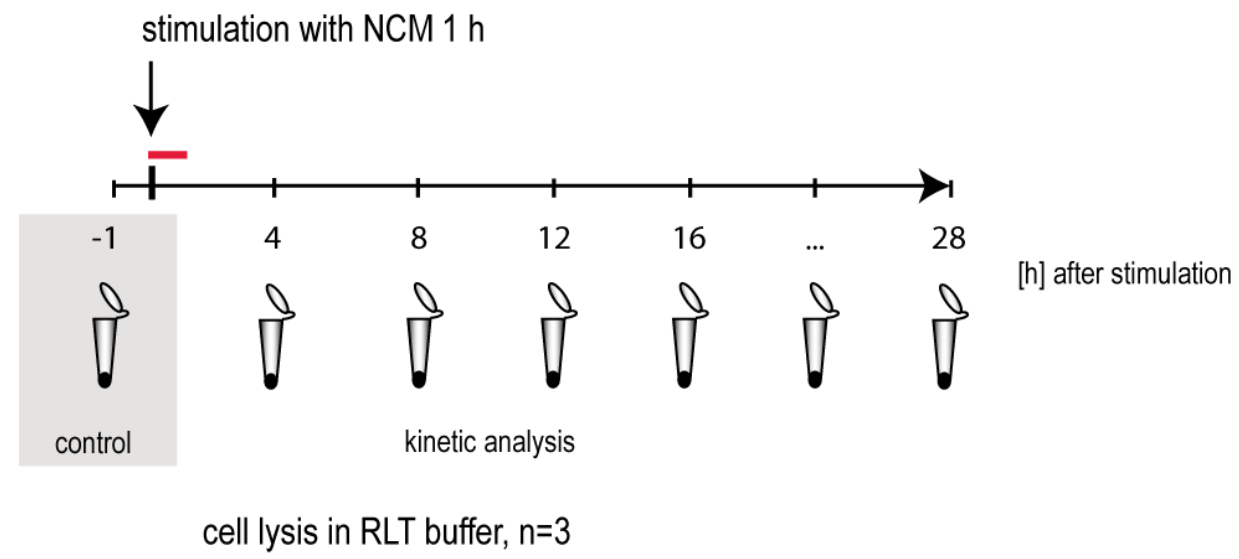

Fig. 18 Experimental design of primary cultured oligodendrocyte stimulation with NCM Primary cultured oligodendrocytes were prepared from the cortices of $10-11 \mathrm{WT}$ and $\mathrm{S} 1 / 2^{-/-}$mice aged P0. After seven DIV, differentiated, Mpb-positive oligodendrocytes were stimulated with NCM for one hour. For the kinetic analysis, cells were harvested in RLT lysis buffer every four hours with $\mathrm{n}=3$ replicates. A native, non stimulated control sample was also taken (indicated in grey). For qRT-PCR analysis the samples were pooled. pOL; primary cultured oligodendrocyte; $\mathrm{P} 0$, postnatal day 0 ; DIV, days in vitro; $\mathrm{NCM}$, neuronal conditioned medium.

Immunofluorescent labelling using the anti-MBP antibody showed differentiated oligodendrocytes in WT and mutant cultures, both before and after stimulation with NCM (Fig. 19A). Quantitative analysis indicated a comparable number of MBP-positive cells in cultures from both genotypes (4.6\% for WT, and $4.8 \%$ for mutant cultures) and an elevated number of GFAP-positive astrocytes in mutant cultures $\left(2.6 \%\right.$ for WT, and $4.3 \%$ for $\mathrm{S} 1 / 2^{-/}$ cultures) (Fig. 19B). Western blotting revealed reduced protein levels for CNP1, PLP, and MBP in mutant cells, whereas MAL levels were unchanged (Fig. 19C-F). 
A
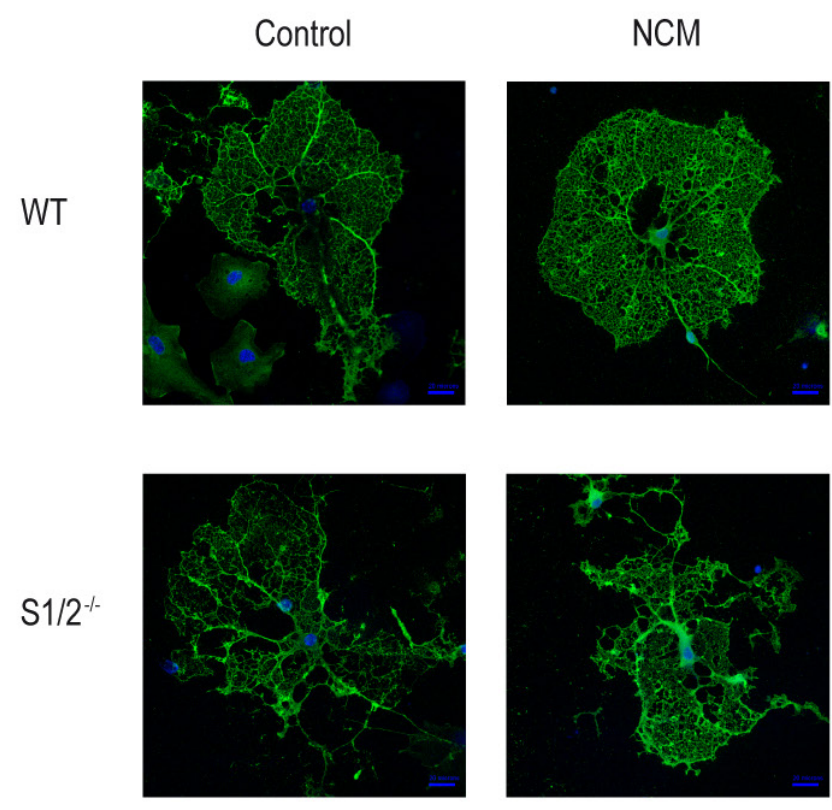

B

\begin{tabular}{|l|c|c|}
\multicolumn{1}{c}{} & \multicolumn{1}{c}{ WT } & \multicolumn{1}{c|}{$\mathrm{S} 1 / 2^{-}$} \\
\hline MBP pos. cells & $4.6 \%$ & $4.8 \%$ \\
\hline GFAP pos. cells & $2.6 \%$ & $4.3 \%$ \\
\hline
\end{tabular}

C

\section{WT $S 12^{1-}$}

CNP1

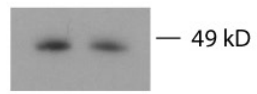

a-Tubulin

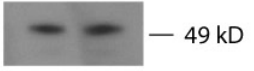

E

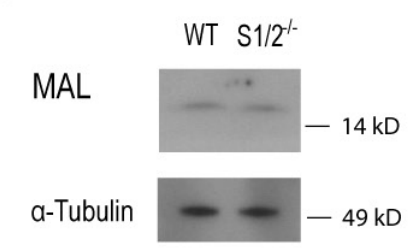

D

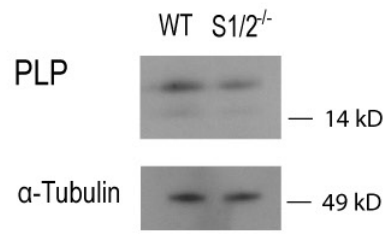

F

MBP

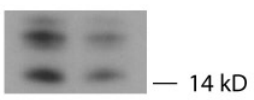

a-Tubulin

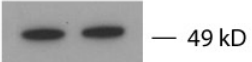

Fig. 19 Immunocytochemical and western blot analysis of oligodendrocyte cultures

(A) Representative pictures of MBP-stained oligodendrocytes in WT and mutant cultures under control and stimulated conditions. (B) Quantitative immunocytochemical analysis revealed a similar number of MBP-positive oligodendrocytes in WT and S1/2/- cultures, with $4.6 \%$ and $4.8 \%$, respectively. GFAPstaining indicated a higher number of GFAP-positive astrocytes in cultures prepared from S1/2 $2^{-/}$mice (4.3\%) compared to WT controls (2.6\%). Western blot analysis showed decreased levels of (C) CNP1 (46 $\mathrm{kD})$, (D) PLP (25 kD), and (F) MBP (18.5 kD) in S1/2 ${ }^{-/}$cultures, whereas levels for (E) MAL (15 kD) were not altered. The anti-CNP antibody recognised CNP1 $(46 \mathrm{kD})$. The anti-MBP antibody identified four different splice variants between $18-20 \mathrm{kD}$. The anti-PLP antibody showed PLP and to a lesser extent its splice variant DM20 (25 and $20 \mathrm{kD}$, respectively). NCM, neuronal conditioned medium; pos., positive; WT, wild type; S1/2/- , SHARP1 and -2 null-mutant. The scale bar indicates $20 \mu \mathrm{m}$. 
As both cultures showed a comparable level of differentiation and contained similar numbers of MBP-positive oligodendrocytes, mRNA expression analysis was performed.

qRT-PCR data were normalised to the housekeeping genes ATp5b and Top1 (Fig. 20A,B), which revealed the most robust time-dependent expression profiles.

The canonical clock marker gene Per2 showed the highest expression in untreated control WT cultures (Fig. 20C). Four hours after adding NCM, a 2.1-fold decay of Per2 mRNA in the WT cultures was observed. Later, the expression remained stable until twelve hours after stimulation, when Per 2 mRNA levels started to increase slightly. In the cultures prepared from the mutant mice, the native control showed comparable expression levels to WT cells. After addition of NCM, Per2 levels slightly increased, followed by a slow decline starting at four hours and continuing until 20 hours after stimulation. Taken together, Per2 levels were elevated in mutant cultures in the kinetic analysis. Two-way ANOVA indicated a significant interaction of genotype and time $\left(\mathrm{F}_{(5,18)}=4.14 ; \mathrm{p}=0.0112\right)$.

Next, the IEG Fos was analysed. In WT samples, Fos transcripts were highest in the native control (Fig. 20D). Four hours after NCM administration Fos levels scored 1.6-fold lower. During the following time course, Fos showed only low variability in expression. In mutant cultures, Fos mRNA abundance was 1.8-fold lower than in WT samples. In the kinetic analysis, Fos was similarly regulated until the 16 hours mark was reached when compared to control mice, and on average, Fos showed an elevated expression level. Two-way ANOVA showed a very significant interaction of genotype and time $\left(\mathrm{F}_{(5,18)}=131.83 ; \mathrm{p}<0.0001\right)$.

Cnp transcripts displayed the highest expression levels in untreated control samples (Fig. 20E). Four hours after NCM stimulation, levels were 1.7-fold reduced in WT controls. During the following analysis, Cnp was mildly induced (during time point 8 and 12) and repressed again. The mutant samples displayed a prominent reduction in Cnp expression at all time points measured. When comparing the native controls of each genotype, Cnp expression in the mutants was almost 2-fold lower. Four hours post NCM, the expression levels only decreased slightly. Markedly, a substantial decrease could be observed at eight hours after NCM application, followed by a mild variability in expression. The Cnp profile in mutant mice was inversely regulated for the first part of the kinetic analysis, but after 16 hours, the expression pattern was similar. Although Cnp levels were reduced in the mutant mice (for most time points between 1.6- and 2-fold), the degree of regulation was comparable. Twoway ANOVA indicated a very significant interaction of genotype and time $\left(\mathrm{F}_{(5,18)}=7.52\right.$; $\mathrm{p}=0.0006)$. 
Plp expression levels were similar for both WT and mutant cultures (Fig. 20F). In the WT native control the maximum of Plp expression was observed. Four hours after NCM application, Plp levels were reduced by 1.4-fold. For the following time points expression levels slightly decreased to the trough at 20 hours post NCM. Of note, mutant mice revealed a reduction of $\mathrm{Plp}$ transcripts for all analysed time points. When comparing native controls of both genotypes, a 2.4-fold reduction of expression in the mutant cultures could be observed. This difference in expression levels remained similar after stimulation with NCM. In the mutant oligodendrocytes, Plp mRNA abundance was 1.5-fold reduced at four hours post NCM. Plp levels, however, showed only minor variability in expression during the kinetic analysis. The degree of regulation appeared to be smaller than in the WT samples. Two-way ANOVA revealed a significant interaction of genotype and time $\left(\mathrm{F}_{(5,18)}=3.72 ; \mathrm{p}=0.0173\right)$.

As already observed for other myelin genes, $M b p$ mRNA levels were highest in the native controls in both cultures (Fig. 20G). Four hours after stimulation with NCM, Mbp expression was reduced 2.2-fold in WT and 1.7-fold in mutant cultures. Furthermore, the expression of $M b p$ was very stable in the WT condition. At eight hours post NCM, a mild induction was monitored, followed by a down-regulation. In mutant cultures, expression of $M b p$ showed almost no variances. During the kinetic analysis, the level of expression was at least 2-fold lower in the mutant cultures. Two-way ANOVA indicated a significant interaction of genotype and time $\left(\mathrm{F}_{(5,18)}=5.67 ; \mathrm{p}=0.0026\right)$.

To study a potential alteration regarding the lipid metabolism, 3-hydroxy-3-methyl-glutarylCoenzyme A reductase (Hmgcr) and Apolipoprotein A-1 (ApoAl) expression were also analysed. HMGCR acts as the rate-limiting enzyme of the mevalonate pathway, by which cholesterol and other isoprenoids are produced. In WT oligodendrocytes, Hmgcr mRNA was highest expressed in non stimulated controls (Fig. 20H). After addition of NCM, a 1.7-fold decrease in expression was observed. At eight hours post NCM the expression was mildly increased, followed by constant expression. Mutant cultures displayed similar kinetics, but showing reduced expression levels, with a range of 1.1- to 1.4-fold. Two-way ANOVA indicated a significant interaction of genotype and time $\left(\mathrm{F}_{(5,18)}=4.96 ; \mathrm{p}=0.0050\right)$.

Apolipoprotein A1 (ApoA1) constitutes the major protein component of high density lipoprotein (HDL) in the blood plasma, promoting cholesterol efflux to the liver for excretion. ApoA1 expression was 1.5-fold reduced in WT oligodendrocytes upon NCM stimulation (Fig. 20I). Like for Hmgcr, a mild peak at eight hours with no variability in regulation was monitored. In S1/2 ${ }^{-/-}$cultures, ApoA1 levels were 1.5-fold less abundant. Furthermore, I 
detected no variances upon stimulation with NCM in mutants, and thus, a very similar kinetic pattern as in WT cells. Two-way ANOVA showed a significant interaction of genotype and time $\left(\mathrm{F}_{(5,18)}=4.01 ; \mathrm{p}=0.0127\right)$.

Next, the astrocyte markers GFAP and S100ß were analysed. Gfap mRNA, expressed by reactive astrocytes, was mildly repressed four hours post NCM (1.2-fold) (Fig. 20J). In addition, the mRNA abundance underwent only subtle variability until 16 hours after NCM, when a 1.3-fold increase was detected. In mutant cultures, Gfap was higher expressed at all time points monitored, except for the last one. Similar to WT cells, Gfap expression was reduced by 1.2-fold four hours post NCM. Taken together, the kinetic profile was reminiscent of the WT pattern, although the extent of regulation was augmented, the last time point being an exception. Two-way ANOVA indicated a very significant interaction of genotype and time $\left(\mathrm{F}_{(5,18)}=10.37 ; \mathrm{p}<0.0001\right)$.

The regulation of $S 100 \beta$, considered to encode a pan-astrocytic marker protein, was very similar between WT and mutant cultures (Fig. 20K). In both cases, a down-regulation was observed four hours post NCM (2-fold for WT, and 1.8-fold for mutant cells), without no obvious variability during the kinetic analysis. Of note, $S 100 \beta$ mRNA was more abundant in WT cultures for all time points (up to 1.8-fold). Two-way ANOVA indicated a significant interaction of genotype and time $\left(\mathrm{F}_{(5,18)}=4.66 ; \mathrm{p}=0.0067\right)$.

Last, expression profiles of Sharp1 and -2 were analysed in WT cultures (Fig. 20L,M). Both genes showed only minor differences in regulation with a similar pattern. Sharpl and -2 expression was higher in the native, non stimulated control in comparison to the first sample after NCM administration (1.4-fold for Sharp1, and 1.2-fold for Sharp2). Expression of both genes was slightly enhanced at eight hours after NCM, and decreased again. For Sharp2, a second induction was scored at the last time point.

In conclusion, major myelin genes including Cnp, $P l p$ and $M b p$ were down-regulated in primary cultures obtained from $\mathrm{S} 1 / 2^{-/-}$mice. Also, the lipid metabolism important for myelin integrity appeared to be reduced in the mutant cells, indicated by lower Hmgcr and ApoAl expression. Of interest, two common astrocytic markers were regulated in an opposite manner. Gfap expression was higher in the mutant cultures, however, it was opposite for $S 100 \beta$. Virtually all genes responded to the addition of NCM, mostly resulting in a repression of expression levels. 
Reference genes

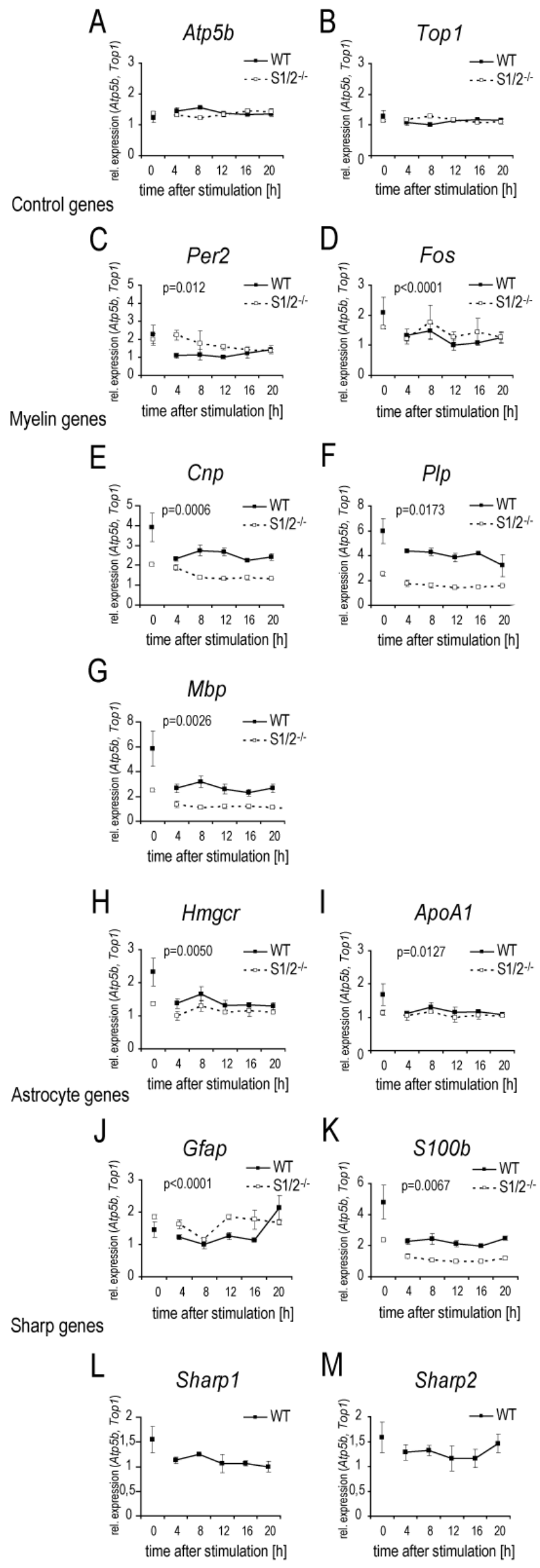


Fig. 20 Dynamic expression of myelin genes in stimulated primary oligodendrocyte cultures and reduced expression levels in $\mathrm{S} 1 / 2^{-/-}$cultures 1

(A,B) qRT-PCR data were normalised to the housekeeping genes Atp5b and Top1, which both revealed stable kinetic expression profiles in WT and mutant cultures. The first time point ' 0 hours' was taken at the beginning of the experiment, representing the gene expression profile in the non stimulated, native culture, as indicated by the dot in the graphs. After stimulation with NCM, samples were taken every four hours. In the graphs, the corresponding qRT-PCR data were connected to reveal the kinetic profile. (C) In WT cells, Per 2 expression went down upon four hours post NCM and revealed no variability despite a slight induction of expression from 12 to 20 hours after NCM. Mutant cultures displayed an up-regulation of Per 2 expression in response to NCM stimulation at the four hour mark, but afterwards, mRNA levels declined until the last time point. Two-way ANOVA indicated a significant interaction of genotype and time $\left(\mathrm{F}_{(5,18)}=4.14 ; \mathrm{p}=0.0112\right)$. (D) Fos transcription in both WT and mutant cultures was reduced four hours post NCM compared to native levels (time point 0). Further on, Fos kinetics were similar between cultures indicating a weak transient peak after eight hours followed by minor differences in expression. Two-way ANOVA showed a very significant interaction of genotype and time $\left(\mathrm{F}_{(5,18)}=131.83 ; \mathrm{p}<0.0001\right)$. Expression analysis of the myelin genes (E) Cnp, (F) Plp and (G) Mbp revealed a down-regulation four hours after NCM in WT and mutant cultures with no further variances in expression (WT cultures showed for $C n p$ and $M b p$ a mild transient peak after eight hours). Strikingly, expression was higher for all genes in WT cultures. Cnp: Two-way ANOVA indicated a very significant interaction of genotype and time $\left(\mathrm{F}_{(5,18)}=7.52 ; \mathrm{p}=0.0006\right) ;$ Plp: Two-way ANOVA revealed a significant interaction of genotype and time $\left(\mathrm{F}_{(5,18)}=3.72 ; \mathrm{p}=0.0173\right) ; M b p$ : Two-way ANOVA indicated a significant interaction of genotype and time $\left(\mathrm{F}_{(5,18)}=5.67 ; \mathrm{p}=0.0026\right)$. Similar to myelin genes, expression of two metabolic genes involved in sterol biosynthesis, (H) Hmgcr and (I) ApoA1, was declined four hours after NCM administration. As observed for $C n p$ and $M b p$ WT conditions, a transient mild peak of mRNA expression appeared at eight hours in WT and mutant cultures. Hmgcr: Two-way ANOVA indicated a significant interaction of genotype and time $\left(\mathrm{F}_{(5,18)}=4.96 ; \mathrm{p}=0.0050\right) ;$ ApoA1: Two-way ANOVA showed a significant interaction of genotype and time $\left(\mathrm{F}_{(5,18)}=4.01 ; \mathrm{p}=0.0127\right)$. (J) Astrocyte marker gene expression analysis indicated a slight downregulation of Gfap four hours post NCM in WT and mutant cells. Further, mRNA levels showed a weak peak at eight hours, followed by an induction of expression (which was stronger in $\mathrm{S} 1 / 2^{-/-}$cells). WT cultures displayed an up-regulation of Gfap at 20 hours. On average, Gfap expression levels were elevated in mutant cultures. Two-way ANOVA indicated a very significant interaction of genotype and time $\left(\mathrm{F}_{(5,18)}=10.37 ; \mathrm{p}<0.0001\right)$. (K) $S 100 b$ underwent a reduction of expression in both cultures four hours after NCM administration. No obvious further variances in expression could be observed. For all time points, mRNA abundance was higher in WT cultures. Two-way ANOVA indicated a significant interaction of genotype and time $\left(\mathrm{F}_{(5,18)}=4.66 ; \mathrm{p}=0.0067\right)$. (L) Sharp1 and (M) Sharp2 appeared to be coregulated (except for the last time point). Expression of both genes was slightly reduced four hours post NCM, without any further variances, except a mild induction of Sharp 2 expression after 20 hours. The lowest value was set to 1 . Data represent mean values \pm SD. Statistical analysis was performed by twoway ANOVA, the p-value depicted in the figures refers to the interaction of genotype and time. WT, wild type; S1/2/-, SHARP1 and -2 double null-mutant; NCM, neuronal conditioned medium; ANOVA, analysis of variance; $\mathrm{SD}$, standard deviation.

\subsubsection{Kinetic 2}

The kinetic experiment was repeated to both analyse the response to NCM administration at later time points and also to validate the expression patterns of the major myelin genes.

For this follow-up experiment, two control samples were taken, a native control before stimulation, as in the experiment before, and another control directly after NCM stimulation to elucidate the short-term effects of NCM application (Fig. 21). The kinetic experiment started 16 hours after stimulation. 


\section{pOL Kinetic II}

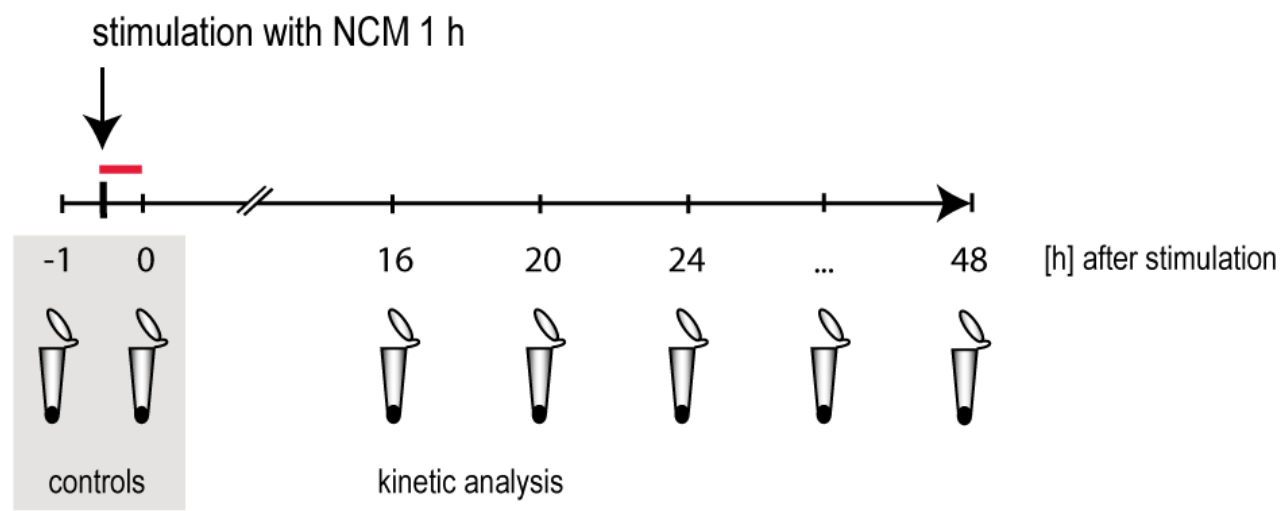

cell lysis in RLT buffer, $n=3$

Fig. 21 Experimental design of primary oligodendrocyte stimulation with NCM

Primary cultured oligodendrocytes were prepared from the cortices of $10-11 \mathrm{WT}$ and S1/2 ${ }^{-/-}$mice aged P0. After seven DIV, differentiated, MBP-positive oligodendrocytes were stimulated with NCM for one hour. For analysis, cells were harvested in RLT lysis buffer every four hours with $n=3$ replicates. Two control samples were taken, one native, non stimulated control and another sample directly after NCM stimulation (indicated in grey). For qRT-PCR analysis the samples were pooled. P0, postnatal day 0; DIV, days in vitro; NCM, neuronal conditioned medium.

Prior to qRT-PCR analysis, WT and mutant primary cultures were characterised by immunocytochemistry (Fig. 22). Both cultures contained differentiated, MBP-positive oligodendrocytes of similar shape and number (7.9\% for WT ans $8.2 \%$ for mutant cultures) (Fig. 22A,B). Also, the cultures showed comparable numbers of oligodendrocytes lineage transcription factor 2 (OLIG2)-positive cells (18.9\% for WT and 18.5\% for mutant cultures), indicating a similar cell number composition. 
A

WT

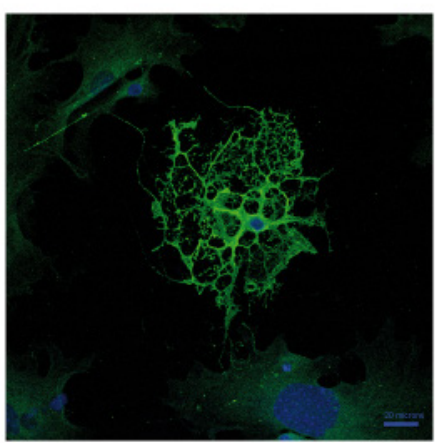

$\mathrm{S} 1 / 2^{-1-}$

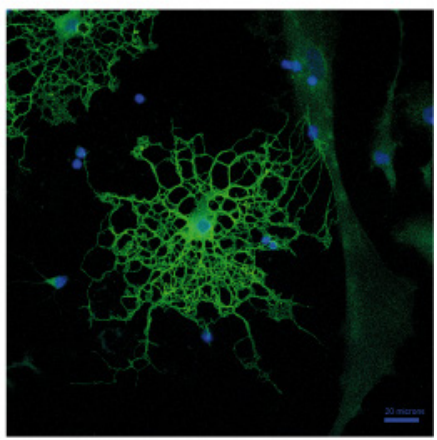

B

\begin{tabular}{|l|c|c|}
\multicolumn{1}{c}{} & \multicolumn{1}{c}{ WT } & \multicolumn{1}{c}{ S1/2 } \\
\hline MBP pos. cells & $7.9 \%$ & $8.2 \%$ \\
\hline OLIG2 pos. cells & $18.9 \%$ & $18.5 \%$ \\
\hline
\end{tabular}

Fig. 22 Immunocytochemical analysis of primary oligodendrocyte cultures

(A) Representative pictures of MBP-stained oligodendrocytes in WT and mutant cultures without NCM stimulation. (B) Quantitative immunocytochemical analysis revealed a similar number of MBP-positive oligodendrocytes in $\mathrm{WT}$ and $\mathrm{S} 1 / 2^{-/}$cultures, with $7.9 \%$ and $8.2 \%$, respectively. OLIG2-staining indicated a comparable number of positive cells in both cultures (18.9\% for WT, and $18.5 \%$ for mutant cultures). NCM, neuronal conditioned medium, pos., positive; OLIG2, oligodendrocyte lineage transcription factor 2; WT, wild type; $\mathrm{S} 1 / 2^{-/}$, SHAPR1 and -2 null-mutant. The scale bar indicates $20 \mu \mathrm{m}$.

qRT-PCR data again were normalised to the housekeeping genes Atp5b and Topl (Fig. $23 \mathrm{~A}, \mathrm{~B})$, as both exhibited a relatively stable expression profile.

In WT cultures, Per2 displayed a meandering pattern after NCM addition. In particular, Per2 increased 1.9-fold within one hour after NCM application by (Fig. 23C). 16 hours after NCM Per 2 mRNA abundance was lower (1.4-fold reduced compared to one hour post NCM). Later on, Per 2 was slightly increased (20 and 24 hours post NCM), then declined, mildly increased again at 40 hours post NCM and went down again. In S1/2/- cultures, Per 2 was downregulated 1.3-fold directly upon application of NCM and was even less abundant at 16 hours post NCM. From 16 to 20 hours after NCM Per2 was induced 1.7-fold, and basically followed the variability observed for the WT cultures (low level of regulation; except time point 40). Per2 mRNA was less abundant in the mutant cultures (1.1- to 1.6-fold difference) 
for control and kinetic samples. Two-way ANOVA indicated a very significant interaction of genotype and time $\left(\mathrm{F}_{(10,33)}=5.08 ; \mathrm{p}=0.0002\right)$.

The IEG Fos was directly up-regulated 1.3-fold in response to NCM in WT cells. However, at 16 hours post NCM Fos levels were strongly reduced (almost 3-fold compared to the time point before) (Fig. 23D). During the kinetic analysis, Fos expression displayed only little variability. In native mutant cultures, Fos mRNA abundance was relatively high (difference to WT native status 1.4-fold enhanced), but almost 2-fold down-regulated at one hour post NCM. At 16 hours after NCM, Fos levels were further reduced similar to the WT level. As in WT cultures, Fos levels displayed only minor differences at later time points. Two-way ANOVA indicated a very significant interaction of genotype and time $\left(\mathrm{F}_{(10,33)}=32.73\right.$; $\mathrm{p}<0.0001)$.

In WT cultures, Cnp mRNA was 1.3-fold induced upon stimulation with NCM at one hour post NCM application, and was even further enhanced after 16 hours post NCM (1.2-fold) (Fig. 23E). At 24 hours, a prominent trough was observed (1.4-fold change), followed by a 1.5-fold induction at 28 hours post NCM. Later, there was a mild peak of expression at 36 hours post NCM, followed by a down-regulation. In mutant cultures, Cnp transcripts showed reduced abundance in the native control (comparison to WT native sample 1.7-fold decreased). This difference between genotypes was further enhanced upon addition of NCM. Immediately after NCM administration, Cnp was down-regulated 1.2-fold in the mutant cultures, which further demonstrated the difference in expression between genotypes. At 16 hours post NCM mRNA levels increased and basically followed the Cnp regulation in the WT cultures with a reduction of expression at 24 hours, continued by an induction that peaked at 36 hours and a further repression. The difference in expression levels between WT and mutant cultures was 1.5- to 1.9-fold. Two-way ANOVA revealed a very significant interaction of genotype and time $\left(\mathrm{F}_{(10,33)}=7.79 ; \mathrm{p}<0.0001\right)$.

The expression pattern of $P l p$ very much resembled the regulation of $C n p$ (Fig. 23F). First, a prominent higher level of Plp transcripts was observed in the control samples when compared to the mutant cultures (after NCM application between 2.6- and 3.7-fold change). In WT cultures, Plp expression was 1.3-fold induced after one hour of NCM. At 16 hours post NCM, Plp mRNA was 3-fold more abundant. Then, Plp transcripts further declined at 24 hours post NCM (1.4-fold), but four hours later increased again (1.6-fold). Like Cnp, the number of Plp transcripts went down towards 44 hours post NCM. The mutant cultures showed a very similar pattern of regulation, although there was a prominent difference in expression 
intensity. Upon administration of NCM there was a mild down-regulation (1.1-fold), but at 16 hours, a substantial induction was observed (2.2-fold). Afterwards, the pattern of regulation was similar to WT samples, but with a lower amplitude. Two-way ANOVA indicated a very significant interaction of genotype and time $\left(\mathrm{F}_{(10,33)}=40.55 ; \mathrm{p}<0.0001\right)$.

Like Cnp and Plp, Mbp mRNA abundance was higher in WT compared to mutant cells (after NCM application between 3.3- to 3.7-fold difference) (Fig. 23G). In addition, the regulation of $M b p$ was reminiscent of Cnp and Plp expression, at least for the first part of the kinetic. In WT cultures, mRNA abundance went up at one hour post NCM and was further enhanced at 16 hours. Mutant cells revealed a transient down-regulation of $M b p$ in response to NCM, whereas after 16 hours, levels appeared to be elevated. Both cultures displayed a similar pattern of regulation, with a trough at 24 hours, followed by a peak at 36 hours (which was not seen for $C n p$ and $P l p$ ). Two-way ANOVA displayed a very significant interaction of genotype and time $\left(\mathrm{F}_{(10,33)}=10.41 ; \mathrm{p}<0.0001\right)$.

Potential changes in lipid metabolism were again examined. Hmgcr analysis indicated a higher expression in the WT cultures, although the native controls did not differ in expression levels (Fig. 23H). Upon administration of NCM however, the difference appeared. In WT cultures, a 1.2-fold increase of expression was seen after NCM stimulation. At 16 hours, however, levels were reduced, remained relatively stable and peaked at 36 hours post NCM (1.4-fold change). Later on, mRNA levels declined 2.2-fold until 44 hours post NCM. In mutant cultures, Hmgcr expression was 1.1-fold repressed upon stimulation of NCM at the one-hour mark, and 16 hours later it was even 1.4-fold decreased. Repeatedly, the mutant profile very much resembled the WT regulation, an induction of gene expression could be observed at 24 hours post NCM, followed by a repression and a second induction at 36 hours post NCM. Two-way ANOVA displayed a very significant interaction of genotype and time $\left(\mathrm{F}_{(10,33)}=11.59 ; \mathrm{p}<0.0001\right)$.

Squalene synthase (Sqs) constitutes an important enzyme for sterol metabolism. On the molecular level, it catalyses the conversion of two molecules of farnesyl pyrophosphate into squalene. In our cultures, $S q s$ expression was mildly enhanced (1.2-fold) one hour post NCM in the WT condition, whereas 16 hours later, the level declined again (Fig. 23I). At 24 hours after NCM application, mRNA levels increased 1.7-fold again and remained relatively elevated until 40 hours, when a decline was observed. In samples prepared from mutant mice, the untreated control displayed a 2.1-fold lower mRNA abundance compared to WT animals. However, Sqs was prominently induced 11-fold at one hour post NCM, whereas at later time 
points during the kinetic analysis, its expression declined and revealed to be predominately lower in the mutant samples. Later, mRNA abundance in mutant mice showed no strong regulation. Two-way ANOVA displayed a very significant interaction of genotype and time $\left(\mathrm{F}_{(10,33)}=38.55 ; \mathrm{p}<0.0001\right)$.

$S 100 b$, a pan-astrocytic marker, was up-regulated in WT samples at all time points monitored (Fig. 23J). The $S 100 \mathrm{~b}$ expression level was not influence by NCM addition, neither in WT, nor in $\mathrm{S} 1 / 2^{-/-}$cultures. In WT cells, a 1.6-fold repression could be observed at time point 24, followed by a strong induction starting at 28 hours post NCM and continuing to the prominent peak of expression at 36 hours after NCM (2.7-fold). Later, mRNA levels rapidly declined. Mutant cultures showed a very similar regulation, after 24 hours post NCM S100b levels began to rise to the maximum of expression at time point 36 (as in the WT cells). However, the extent of regulation was smaller, displaying a 1.5-fold change only. Like in WT cultures, the expression also declined in mutant cells at later time points. Two-way ANOVA displayed a very significant interaction of genotype and time $\left(\mathrm{F}_{(10,33)}=38.55 ; \mathrm{p}<0.0001\right)$.

Last, Sharp gene expression in WT samples was assessed (Fig. 23K,L). Sharp1 and Sharp2 were co-regulated, as both genes were induced upon application of NCM (1.3-fold for Sharp1, and 1.2-fold for Sharp2). At time point 16, Sharp gene expression was repressed, but later was again induced and remained at an elevated level.

In conclusion, the data from the first in vitro stimulation experiment were validated with this second, independent experiment. Both, myelin genes (Cnp, Plp and $M b p$ ) and genes involved in lipid metabolism (Hmgcr, Sqs) were expressed to a lesser extent in mutant cultures. The same was true for $S 100 \mathrm{~b}$. However, the data of the second experiment indicate a stronger gene regulation. For most genes, the pattern was similar between WT and mutant cultures. Cnp, Plp and to some extent Mbp again appeared to be co-regulated. Of note, Hmgcr and S100b revealed a similar regulation with a prominent peak at time point 36 . 
Reference genes

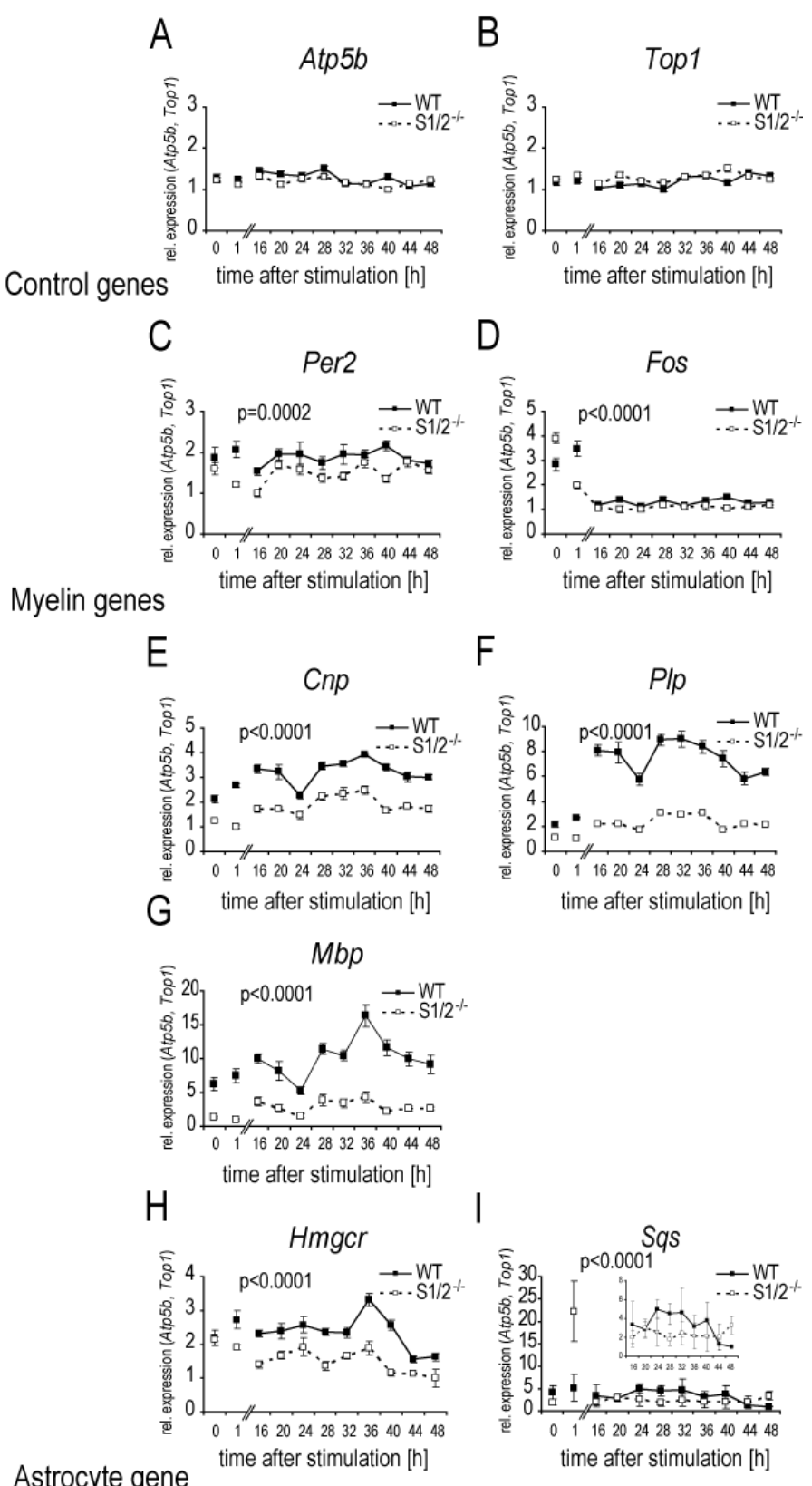

Astrocyte gene
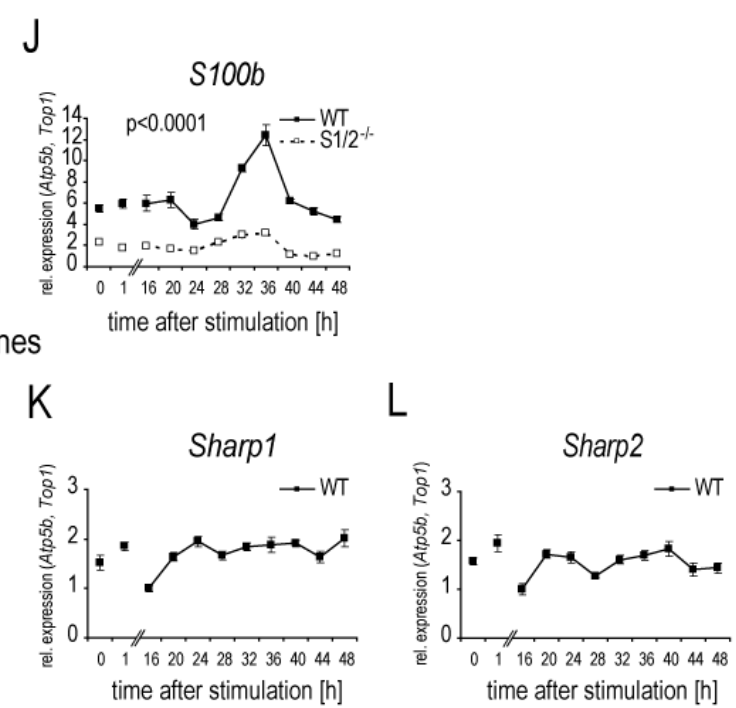
Fig. 23 Dynamic expression of myelin genes in stimulated primary oligodendrocyte cultures and reduced expression levels in $\mathrm{S} 1 / 2^{-/-}$cultures 2

(A,B) qRT-PCR data were normalised to the housekeeping genes Atp5b and Top1 which both showed a stable kinetic expression profile. Two control samples were taken. The first time point ' 0 hours' represents the non stimulated, native culture, the other control was taken immediately after NCM stimulation ' 1 hour' as represented by the dots in the graphs. After stimulation with NCM, 16 hours was waited until the kinetic analysis started. In the graphs, the corresponding qRT-PCR data were connected to reveal the kinetic profile. (C) The clock gene Per2 in WT cultures appeared to be mildly up-regulated one hour after NCM, followed by a down-regulation 16 hours later. Per 2 kinetic analysis displayed moderate differences in regulation. In mutant cultures, Per 2 mRNA decreased one hour after NCM application and even further at time point 16 . Like in WT samples, Per2 showed moderate cycling behaviour. Two-way ANOVA indicated a very significant interaction of genotype and time $\left(\mathrm{F}_{(10.33)}=5.08\right.$; $\mathrm{p}=0.0002$ ). (D) Fos mRNA in WT cultures increased one hour after stimulation with NCM, whereas in mutant mice a decrease could be observed. 16 hours later, the levels were low in both genotypes and showed only minor variability in expression. Two-way ANOVA indicated a very significant interaction of genotype and time $\left(\mathrm{F}_{(10,33)}=32.73 ; \mathrm{p}<0.0001\right)$. The myelin genes (E) Cnp, (F) Plp and (G) Mbp showed higher expression levels in WT cultures for all monitored time points. The regulation was similar among genes and genotypes. In WT cultures, NCM led to an increase of expression, whereas in the mutant cells a decrease was observed. 16 hours later, levels further increased and were repressed at time point 24, enhanced again and to the end again declined. For $M b p$, a prominent peak of expression in WT cultures after 36 hours could be observed. Cnp: Two-way ANOVA revealed a very significant interaction of genotype and time $\left(\mathrm{F}_{(10,33)}=7.79 ; \mathrm{p}<0.0001\right)$; Plp: Two-way ANOVA indicated a very significant interaction of genotype and time $\left(\mathrm{F}_{(10,33)}=40.55 ; \mathrm{p}<0.0001\right)$. Mbp: Two-way ANOVA displayed a very significant interaction of genotype and time $\left(\mathrm{F}_{(10,33)}=10.41 ; \mathrm{p}<0.0001\right)$. $(\mathrm{H}) \mathrm{Hmgcr}$ expression was also higher in WT cells. The pattern of regulation between cultures was similar, a mild peak was observed at time point 24 and a stronger one at 36 hours post NCM. Two-way ANOVA displayed a very significant interaction of genotype and time $\left(\mathrm{F}_{(10,33)}=11.59 ; \mathrm{p}<0.0001\right)$. (I) $S q s$ also was higher expressed in WT samples for almost all time points. A strong peak of expression could be seen in mutant cultures one hour post NCM. Further, only moderate variability in expression was shown. Two-way ANOVA displayed a very significant interaction of genotype and time $\left(\mathrm{F}_{(10,33)}=12.36 ; \mathrm{p}<0.0001\right)$. (J) S100b expression resembled that of $H m g c r$, indicating a strong peak at 36 hours post NCM in WT and mutant samples. To note, mRNA abundance again was higher in WT cells. Two-way ANOVA displayed a very significant interaction of genotype and time $\left(\mathrm{F}_{(10,33)}=38.55 ; \mathrm{p}<0.0001\right)$. (K,L) Sharp1 and -2 expression analysis showed a mild induction of both genes one hour post NCM, followed by a down-regulation 16 hours later. Both genes were subsequently induced and further displayed minor variances in expression. The lowest value was set to 1 . Data represent mean values \pm SD. Statistical analysis was performed by twoway ANOVA, the p-value depicted in the figures refers to the interaction of genotype and time. WT, wild type; S1/2-- SHARP1 and -2 double null-mutant; NCM, neuronal conditioned medium; ANOVA, analysis of variance; $\mathrm{SD}$, standard deviation.

\subsection{Reporter gene assays}

\subsubsection{Repressive and co-activating functions of SHARP1 and -2 functions in a context- specific manner}

In this thesis, the potential dual role of the SHARP proteins on CLOCK/BMAL1- and NPAS2/BMAL1-mediated activities was analysed in different heterologous cell lines and primary cultured neurons (Fig. 24A-C). Reporter gene assays were performed using a firefly luciferase reporter driven by canonical CACGTG E-box enhancer elements. SHARP1 and -2 transfected individually in HEK293 cells significantly repressed reporter activation by 9.4and 4.8-fold, respectively $(\mathrm{p}<0.01$ ) (Fig. 24A). In contrast, co-transfection of NPAS2 and

BMAL1 substantially induced reporter gene transcription by roughly 8.5 -fold. The 
NPAS2/BMAL1-mediated increase in promoter activity was repressed by SHARP1 and -2 proteins 3- and 2.6-fold, respectively $(\mathrm{p}<0.01)$. An identical experiment was repeated in PC12 cells (Fig. 24B), confirming the repressive functions of SHARP1 and -2 on basal transcriptional reporter activity (7- and 1.6-fold respectively, with $\mathrm{p}<0.01$ ). To test whether combinatorial transfections of NPAS/BMAL1 and SHARP1/2 would elicit the same transcriptional responses as observed in HEK293 cells, also this experimental setup was repeated in PC12 cells. Co-transfection of NPAS2/BMAL1 again resulted in robust transcriptional reporter activation (11-fold). Additional co-transfection of SHARP1 displayed repressive functions $(\mathrm{p}<0.01)$, as seen in HEK293 cells. However, co-transfecting SHARP2 led to a modest increase in reporter gene activation (factor 1.1 compared to basal activity $(\mathrm{p}<0.05))$. To test the transcriptional activity in a more relevant cellular model, primary cortical neurons were used for these reporter gene assays (Fig. 24C). Again, SHARP1 and -2 reduced basal reporter activity between 2.5- (SHARP1) and 2.9- fold (SHARP2), with $\mathrm{p}<0.01$. However, both SHARP proteins further increased reporter activity by 2 - to 2.3 -fold, respectively with $\mathrm{p}<0.05$, when NPAS2/BMAL1 were co-transfected.

To elucidate the dual functions of the SHARP proteins, expression constructs lacking the basic (b) region ( $`$ b mutants) were generated by site-directed mutagenesis (Fig. 24D). When this region is deleted, SHARP proteins loose the DNA-binding ability to E-box elements. Reporter gene assays in HEK 293 and PC12 cells confirmed that SHARP $1^{\wedge} \mathrm{b}$ and $-2^{\wedge} \mathrm{b}$ proteins lost the repressive effects on E-box-mediated reporter gene transcription when transfected separately (Fig. 24E-H). In HEK293 cells, SHARP1 and -2 repressed basal reporter gene activity 9.4- and 3.1-fold, respectively $(\mathrm{p}<0.01)$, whereas the repressive effect was strongly reduced for SHARP1^ $\mathrm{b}$ (1.3-fold repression), and resulting in a further 1.4-fold increase in activity upon transfection of SHARP2^b (Fig. 24E). Native constructs of SHARP1 and SHARP2 in PC12 cells revealed a relatively strong repressive function for SHARP1 (7.1-fold, $\mathrm{p}<0.01)$ and a weaker but still significant repressive action for SHARP2 $(1.3$-fold, $\mathrm{p}<0.05)$ (Fig. 24F). These effects were lost upon transfection of mutated constructs. When cotransfecting, the SHARP1^b construct further enhanced NPAS2/BMAL1-mediated reporter gene activation in HEK293 cells by factor $1.6(\mathrm{p}<0.01)$, whereas SHARP2^b had no effect (Fig. 24G). In PC12 cells both mutated constructs significantly enhanced NPAS2/BMAL1dependent reporter gene activation (2-fold increase for SHARP1^ $\mathrm{b}$ and 1.6-fold increase for $\mathrm{SHARP}^{\wedge}$ b, with $\mathrm{p}<0.01$ ) (Fig. $24 \mathrm{H}$ ). These reporter gene assays performed in HEK293 cells, PC12 cells, and cortical neurons were published in (Rossner et al., 2008). 
A

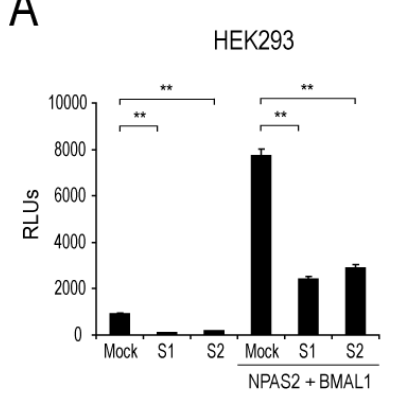

D

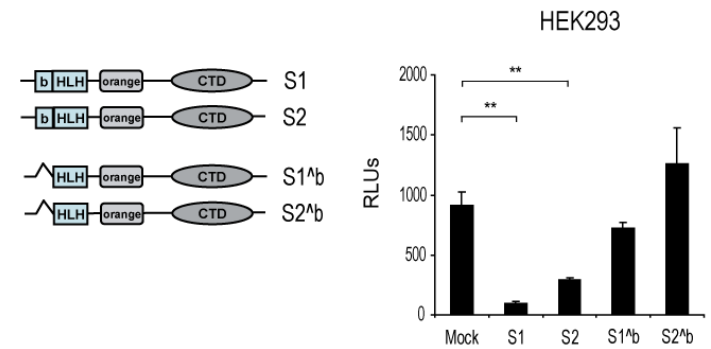

G

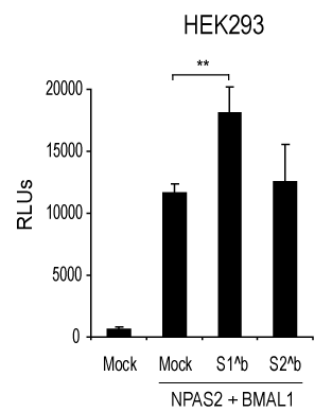

C
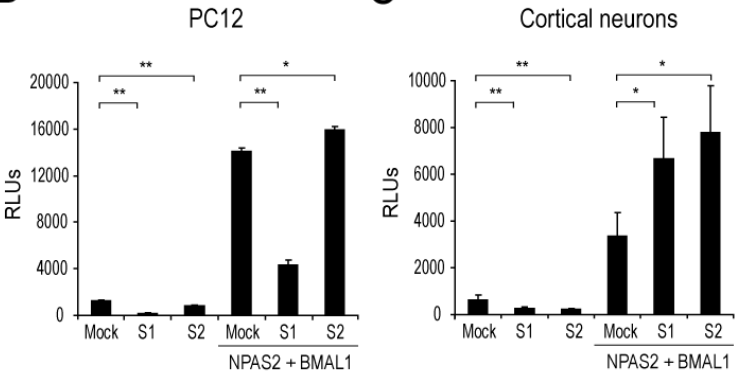

$\mathrm{F}$

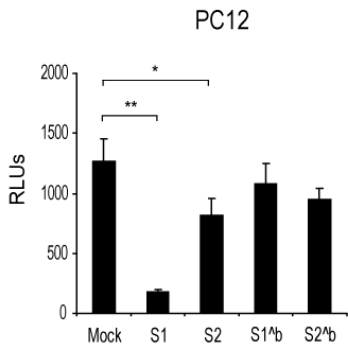

$\mathrm{H}$

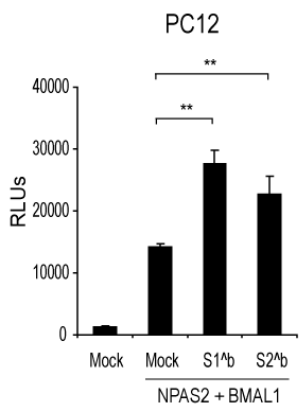

Fig. 24 Repressive and co-activating functions of SHARP1 and $\mathbf{- 2}$ in a context-specific manner (A-C) Reporter gene assays using a firefly luciferase reporter construct driven by a herpes simplex thymidine-kinase (TK) minimal promoter and three upstream clustered CACGTG E-box elements and BMAL1, NPAS2, SHARP1 and SHARP2 expression plasmids were performed in (A) HEK293 cells, (B) PC12 cells and (C) primary cultured cortical mouse neurons as indicated. (A) In HEK293 cells, SHARP1 and -2 repressed basal promoter activity $(\mathrm{p}<0.01$ for SHARP1 and -2$)$. Co-transfection of NPAS2 and BMAL1 encoding plasmids resulted in an enhanced reporter activity. SHARP1 and -2 efficiently repressed this NPAS2/BMAL1-mediated reporter gene activation $(\mathrm{p}<0.01)$. (B) In PC12 cells, SHARP1 transfection repressed basal and NPAS2/BMAL1-dependent reporter activation $(p<0.01)$, whereas SHARP2 exerted repressive functions on basal reporter activity $(\mathrm{p}<0.01)$ but further enhanced NPAS2/BMAL1-mediated activation $(\mathrm{p}<0.05)$. (C) In primary cultured neurons, SHARP1 and -2 repressed basal promoter activity $(\mathrm{p}<0.01)$, but further co-activated NPAS2/BMAL1-driven reporter activation $(\mathrm{p}<0.05$ ). (D) Domain structures of SHARP1 and -2 full-length constructs and deletion mutants SHARP $1^{\wedge} b$ and SHARP2 ${ }^{\wedge} b$ lacking the basic domain. (E-F) Reporter gene assays using a firefly luciferase reporter construct driven by a TK minimal promoter and three upstream clustered CACGTG EBox elements and BMAL1, NPAS2, SHARP1, SHARP2, SHARP1^ $b$ and SHARP2^ $b$ expression plasmids were performed in HEK293 cells (E,G) and PC12 cells (F,H), as indicated. Full-length SHARP1 and -2 repressed basal reporter activity in HEK293 and PC12 cells when transfected alone, with $\mathrm{p}<0.01$ for SHARP1 for both cell lines and $\mathrm{p}<0.01 / \mathrm{p}<0.5$ for SHARP2 in HEK293 and PC12 cells, respectively. SHARP $1^{\wedge} \mathrm{b}$ and $-2^{\wedge} \mathrm{b}$ deletion mutants showed no significant repressive effect. Upon co-transfection of NPAS2/BMAL1, SHARP1^ $b$ and $-2^{\wedge} b$ deletion mutants further acted as co-activators in HEK293 $\left(\mathrm{p}<0.01\right.$ for SHARP $\left.1^{\wedge} \mathrm{b}\right)$ and PC12 cells $\left(\mathrm{p}<0.01\right.$ for SHARP $-1^{\wedge} \mathrm{b}$ and SHARP $\left.2^{\wedge} \mathrm{b}\right) .{ }^{*}, * *$ and $* * *$ indicate $\mathrm{p}<0.05, \mathrm{p}<0.01$ and $\mathrm{p}<0.001$, respectively. Data represent mean values \pm SD for $\mathrm{n}=6$ (HEK and PC12 cells) and $n=12$ (neurons) replicates. Significance values refer to the Mann-Whitney test. S1, SHARP1; S2, SHARP2; RLUs, relative luciferase units; B, basic domain; HLH, helix-loop-helix domain; orange, orange domain, CTD, C-terminal domain. 


\subsubsection{Effects of SHARP proteins on myelin promoter activity in vitro}

\subsubsection{SHARP2 enhances myelin promoter activity in stably transfected Oli-neu cell lines}

Stably transfected Oli-neu lines were generated expressing the firefly luciferase reporter gene under control of the $1.5 \mathrm{~kb}$ DNA region proximal to the $\mathrm{Mal}$ gene coding region (Fig. 25A) or of the $1.3 \mathrm{~kb}$ DNA region proximal to the $M b p$ gene coding region (Fig. 25E), respectively. To elucidate the role of SHARP2 on myelin gene expression, a SHARP2 expressing lentivirus was cloned to infect the stable cell lines.

The response of three different Oli-neu cell lines was analysed for each reporter plasmid. SHARP2 significantly enhanced reporter gene activity of the Mal promoter in all three lines (Mal promoter clone 10: 1.6-fold, with $\mathrm{p}<0.0001$; Mal promoter clone 12: 1.8-fold, with $\mathrm{p}<0.0001$; Mal promoter clone 22: 1.8-fold, with $\mathrm{p}<0.0001$ ) (Fig. 25B-D). Mbp promoter activity was significantly increased by SHARP-2 in the Mbp clone 4: 1.5 -fold, with $\mathrm{p}<0.0001$ (Fig. 25F). However, the other two analysed $M b p$ promoter clones only showed minor enhanced firefly expression levels upon SHAPR2 infection (Mbp clone 10: 1.03-fold, with $\mathrm{p}=0.4642 ; M b p$ clone 18: 1.01-fold, with $\mathrm{p}=0.8148)($ Fig. 25G,H). 
A

B

C

Mal prom clone 10

Mal prom clone 12

Mal prom clone 22
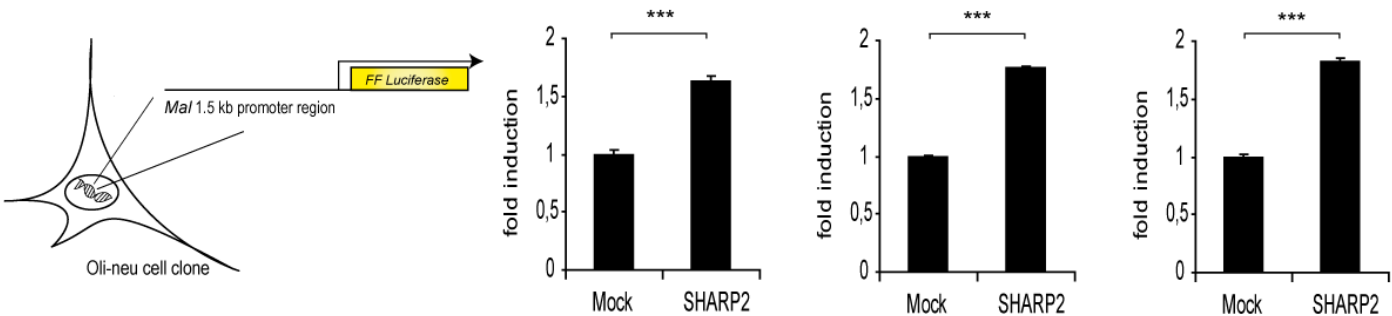

E

F

G

$\mathrm{H}$

Mbp prom clone 4

Mbp prom clone 10

Mbp prom clone 18
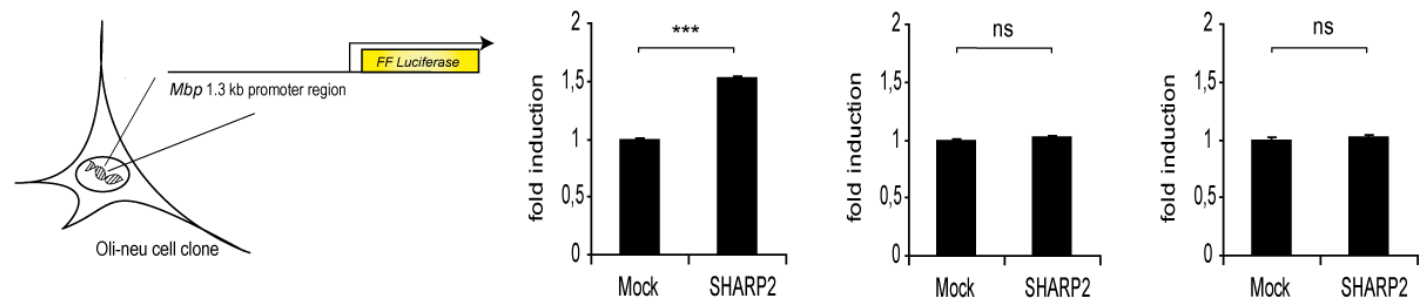

Fig. 25 SHARP2 increases myelin promoter activity in stably transfected Oli-neu cell lines

Schematic representation of stably transfected Oli-neu lines expressing the FF luciferase reporter gene under control of (A) the 1.5 proximal region of the Mal promoter or (E) the 1.3 proximal region of the $M b p$ promoter. (B-D) Infection with a SHARP2 expressing lentivirus resulted in a significant increase of the $\mathrm{Mal}$ promoter activity in all three tested lines to a similar extent (with $\mathrm{p}<0.0001)$. (F-H) At least one $M b p$ promoter lines also showed a significant increase in reporter gene activity upon SHARP2 expression (F), with $\mathrm{p}<0.0001$, whereas the two other clones almost displayed no difference to the mock control $(\mathrm{G}, \mathrm{H})$. Data represent mean values $\pm \mathrm{SD}$ for $\mathrm{n}=24$ technical replicates. Mock controls are set to 1 and fold induction relative to Mock control is displayed. Significance values refer to the Mann-Whitney test. Ns, non significant; $* * *$ indicates $\mathrm{p}<0.001$. Statistical analysis was performed with the Mann-Whitney test. $\mathrm{FF}$, firefly; prom, promoter; SD, standard deviation.

\subsubsection{SHARP enhances myelin promoter activity in primary cultured oligodendrocytes}

To investigate whether SHARP1 and -2 could influence myelin promoter activity by exerting co-activating or repressing functions, a $1.5 \mathrm{~kb}$ DNA region proximal to the Mal gene coding region was amplified and cloned into a promoter-less firefly luciferase reporter vector. Accordingly, co-transfection of SHARP1 and -2 induced a significant increase in Mal promoter activity in primary cultured oligodendrocytes when compared to basal levels (Mock) (3-fold increase, $p<0.0001$ ) (Fig. 26). 


\section{Cortical oligodendrocytes}

Mal promoter activity

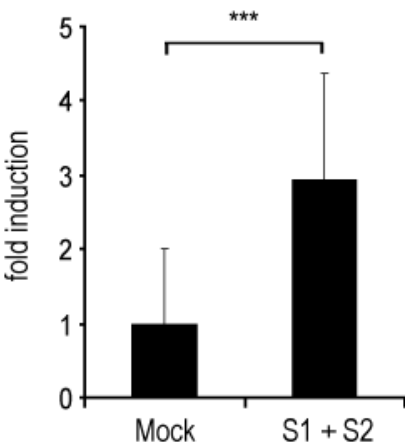

Fig. 26 SHARP2 increases myelin promoter activity in stably transfected Oli-neu cell lines

Compared to basal $\mathrm{Mal}$ promoter activity luciferase levels were significantly increased to nearly 3-fold when SHARP1 and -2 were co-transfected $(\mathrm{p}<0.001)$. Data represent mean values \pm SD for $n=24$ technical replicates. Mock controls are set to 1 and fold induction relative to Mock control is displayed. Significance values refer to the Mann-Whitney test. $* * *$ indicates $p<0.001$. Statistical analysis was performed with the Mann-Whitney test. FF, firefly; S1, SHARP1; S2, SHARP2; SD, standard deviation. 


\section{DisCUSSION}

\subsection{Behavioural alterations reminiscent of psychiatric diseases in $\mathrm{S} 1 / 2^{-/-}$ mice}

SHARP1 and -2 double null-mutant $\left(\mathrm{S} 1 / 2^{-/}\right)$mice were analysed in behavioural tests assessing motivation-, exploratory-, curiosity- and anxiety-related behaviour that might be important in the context of psychiatric diseases. Mutant mice showed novelty-induced hyperactivity and decreased anxiety levels in the open field test. The open field box was subsequently equipped with a 'hole board' to assess curiosity-related behaviour. Mutant mice displayed no difference in activity in this more familiar context, but exploratory behaviour was decreased. Also, the tail suspension test indicated a trend to enhanced motivation in mutant mice. These phenotypes are accompanied with hypoactivity over 24 hours that corresponds to the relative increase of sleep of $\mathrm{S} 1 / 2^{-/-}$mice in the dark phase (Shahmoradi et al., in submission). Intriguingly, mutant mice lacking functional CLOCK or NPAS2 proteins display hyperactivity and reduced sleep in the dark phase (Dudley et al., 2003; Roybal et al., 2007). These phenotypes have been described as mania-like behaviour in CLOCK mutant mice (Roybal et al., 2007). Hypoactivity and elevated levels of sleep in the dark phase could be associated to depression-like behaviour of $\mathrm{S} 1 / 2^{-/-}$mice. However, open field and tail suspension tests indicated an increased activity and reduced anxiety compared to WT mice. Mixed-state or paradoxical phenotypes of mania- and depression-associated behaviour are already known from mouse mutants, for example, when Clock was selectively knocked down in the ventral tegmental area (Mukherjee et al., 2010). Phenotypes of $\mathrm{S} 1 / 2^{-/-}$mice are reminiscent of mixed-state criteria towards bipolar disorder similar to Clock mutant mice (Nestler and Hyman, 2010). Wake-induced expression of transcripts for neural activity/plasticity genes was completely attenuated in $\mathrm{S} 1 / 2^{-/-}$mice (Shahmoradi et al., in submission). Network analysis indicated a link between neural plasticity and several clock genes (among them Perl and Sharp1), which were deregulated in the frontal cortex of schizophrenic patients. These data strongly suggest that SHARP1 and PER1 could be important factors integrating circadian- and plasticity-dependent processes in the human brain.

Intriguingly, oligodendrocyte/myelin specific genes were identified among sleep-wake dysregulated transcripts in the cortex of mutant mice (Shahmoradi et al., in submission). This 
fact was of particular interest, as myelin genes are known to be deregulated in psychiatric diseases such as bipolar disorder (Tkachev et al., 2003), depression (Aston et al., 2005) and schizophrenia (Georgieva et al., 2006; Tkachev et al., 2003). The main objective of this study was to analyse a potential dynamic (day time- and/or activity-dependent) regulation of myelin genes in WT mice as well as an attenuated regulation in $\mathrm{S} 1 / 2^{-/-}$animals in precisely defined grey and white matter micro-areas as well as CNS and PNS nerves.

\subsection{Dynamic regulation of myelin genes in WT mice}

Gene expression profiles of distinct grey and white matter micro-areas were analysed. In particular, the anterior cingulate gyrus (ACC), the somatosensory/motor cortex (SSMCx) and the corpus callosum (CC) micro-region were addressed (see Table 2). The ACC micro-area was chosen because of its fundamental role in higher-order cognitive processing and its association with schizophrenia (Carter et al., 1997; Höistad et al., 2009). The SSMCx area was analysed to rule out any potential effects of enhanced sensory, cognitive and motor stimulation associated with enriched environment housing (Nithianantharajah and Hannan, 2006). It also served as a control to validate the findings related to the ACC micro-region.

To ensure optimal technical performance, expression analysis for both the canonical clock gene Per2 and the immediate-early gene (IEG) Fos was always performed first. Per2 is known to be influenced by time and activity (Cirelli et al., 2004); its expression is described for neurons and astroglia (Jackson, 2011; Prolo et al., 2005). Fos is considered to couple neuronal activation to gene expression, therefore it was used as an indicator of neuronal activity (Bullitt, 1990; Dragunow and Faull, 1989). Although Fos might be expressed as an IEG in glia in response to external stimuli, the strongest signals are found in neurons (compare with Allen Brain Altas; http://www.brain-map.org). In both grey matter areas, Per2 and Fos expression peaked in the dark phase, in most cases at zeitgeber time (ZT)16, which is consistent with published data. Per2 expression showed no differences between genotypes, indicating a functional core clock in mutant mice, which is in line with published data (Bode et al., 2011; Rossner et al., 2008). In both areas, Fos expression was elevated in the dark phase. For the first time it was observed that myelin genes display a dynamic day timedependent regulation following activity markers such as Per2 and Fos. Further, myelin gene regulation was compared between white and grey matter. The corpus callosum is an important structure regarding inter-hemispheric communication. The selected CC micro-region belonged to the 'body' part which connects primary and secondary sensorimotor areas (see 
Fig. 9) and is supposed to contain mainly large, fast-conducting, highly myelinated fibres (Aboitiz and Montiel, 2003). Per2 expression in the CC micro-area was enhanced in the dark phase at ZT16 in both genotypes. However, Fos regulation appeared to be relatively weak, potentially due to the lack of neurons in white matter, which mainly constitute the source of Fos expression (in white matter, only some scattered interstitial neurons are present (Riederer et al., 2004)). For WT mice, the maximum Fos mRNA abundance was observed in the dark phase, at ZT16. Also in the CC micro-area, myelin genes were regulated in a time-dependent diurnal manner. In contrast, however, to the grey matter areas, Cnp, Plp, Mbp, and Mal transcripts were down-regulated at the light-dark transition from ZT10 to ZT16, thereby opposing the expression of the core clock gene Per2 and the IEG marker Fos. The kinetic profiles were validated for Cnp and Plp in an independent, age-matched WT cohort. Next, the sciatic nerve (belonging to the PNS) and the optic nerve (being part of the CNS) were studied to analyse potential region-specific differences.

In the sciatic nerve, the amplitude of Per2 expression was huge in both genotypes, with a peak at ZT10. Compared to the brain micro-regions, the kinetics of Per2 expression appeared to be slightly earlier. It is well known that non-SCN clocks display a tissue-specific phase of clock gene regulation (Honma et al., 1998; Masubuchi et al., 2000), most likely dependent on behavioural rhythms (Masubuchi et al., 2000). As Per2 is known to be influenced by activity as well the time of day (Cirelli et al., 2004), the earlier kinetics might reflect the response of the sciatic nerve to the anticipatory wheel running behaviour of mice that usually starts already at the end of the light phase (compare wheel running profiles in Shahmoradi et al., in submission). However, Per2 also displayed high expression levels at ZT16, correlating with the active phase of mice. Fos mRNA regulation was lower than in the cortical micro-areas, potentially due to the lack of neurons in the sciatic nerve. In WT mice, the maximum of expression correlated with the dark phase. Myelin gene expression analysis only showed mild dynamic regulation for Cnp and Plp in WT animals with a weak peak in the dark phase at ZT16. However, Mal expression pattern and its extent of regulation were different. To conclude, myelin genes in the sciatic nerve were only mildly regulated in comparison to the brain areas. But still, a tendency of elevated expression levels in the active phase was seen, reminiscent of Fos regulation.

To elucidate whether myelin gene regulation is coupled to neuronal activity and/or the circadian time the optic nerve was analysed under light dark (LD) and dark dark (DD) conditions. The hypothesis was that light input in LD would result in an increased neuronal 
activity, which in turn might influence myelin gene expression, an effect that could potentially differ from DD conditions. In summary, no prominent difference between the LD and DD experiment could be detected. In both conditions, Per2 expression peaked at ZT16. To understand, however, Fos expression, a more detailed analysis is needed. In WT mice, highest mRNA abundance was seen at ZT4, followed by a down-regulation at ZT10 and slightly increasing levels during the dark phase. In the DD experiment, Fos was not regulated, and the peak at ZT10 in LD was completely abolished. As already observed in the sciatic nerve, myelin gene regulation was mild, with minor differences between LD/DD experiments. For Mal, again a rather strong regulation was measured supporting the notion that Mal is differently regulated than the other myelin genes monitored.

Due to the absence of differences between the LD and DD rhythm, the following scenarios are suggested. First, the optic nerve activity might be uncoupled from light conditions and more dependent on the behavioural state of the animals, which is similar in LD and DD rhythms. Second, it is known that the optic nerve comprises a heterogeneous group of fibres, including melanopsin containing RGCs that are not involved in image formation, and are, however, rather sensitive to light intensity (Sehgal, 2004). Therefore, the optic nerve activity may not display a clear difference with respect to light conditions. To conclude, for the first time myelin genes were shown to be highly plastic on mRNA level. Thereby, myelin gene regulation appeared to be time- and region-specific.

\subsubsection{Dynamic day time-dependent regulation}

Having identified a dynamic, day time-dependent myelin gene regulation the question arose if glia, specifically oligodendrocytes, express clock genes which might cause periodic myelin gene expression. In Drosophila, it has been known for a long time that glial cells express the clock gene per (Siwicki et al., 1988), and a link between glial per expression and rhythmic behaviour in flies has also been suggested (Ewer et al., 1992). In 2007, a factor important for normal circadian locomotor activity in Drosophila was identified in glia (Emery and Freeman, 2007; Suh and Jackson, 2007). Ebony, a glial N-ß-alanyl-biogenic amine synthetase, is supposed to modulate dopaminergic function in neurons, and thus regulating locomotor activity. The first evidence for an astrocytic expression of clock genes (Perl and Per2) in rodents came from a study by Prolo and colleagues (Prolo et al., 2005). Here, a luciferase reporter gene was placed under the control of Per1 and Per2 promoter sequences, and transgenic animals were generated. In cultured cortical astrocytes from these animals the 
authors described a rhythmic reporter expression for up to one week. Glial rhythms could be shifted and entrained to a physiologically related cycle. To conclude, there is evidence for clock gene expression in astrocytes, however, nothing is known about a molecular clockwork in oligodendrocytes so far. Therefore, a non-cell autonomous mechanism could potentially control the dynamic circadian myelin gene regulation in oligodendrocytes.

Next, potential reasons underlying the dynamic regulation of myelin genes will be addressed. In recent years, many studies have reported that adult plasticity of white matter tracts can be influenced in response to training of skills (Bengtsson et al., 2005; Carreiras et al., 2009; Quallo et al., 2009; Scholz et al., 2009). Therefore, I speculate that the finding of a novel adult day time-dependent plasticity of myelin gene expression supports the current view of myelin being modifiable by experience, thereby potentially contributing to some aspects of learning (Fields, 2008).

On myelin protein level, no circadian differences in the cortex and the corpus callosum could be detected, likely due to a very low turnover of myelin proteins (much longer than 24 hours) (Lajtha et al., 1977; Singh and Jungalwala, 1979). Also, it might be possible that single events of new protein incorporation and/or degradation could not be detected due to the high abundance of myelin proteins in relatively large tissues areas that were taken for analysis. However, Wake and colleagues described that MBP is locally synthesised in oligodendrocytes in close proximity to active axons (Wake et al., 2011). This recent finding is of importance for my current model stating that the dynamic expression of myelin genes can be regarded as an indicator to maintain adult plasticity. Myelin genes might be rhythmically expressed to ensure responsiveness to neuronal activity. As the constant high expression of these genes would require too much of the cell's energy I speculate that the expression is subjected to circadian and/or activity-dependent regulation. There is evidence in the literature pointing towards a potential dynamic regulation of myelin genes, as an array study analysed wake- and sleepdependent gene expression in the whole rat cortex. Among sleep-related transcripts a large group of genes coding for myelin structural proteins (e.g. myelin-associated oligodendrocyte basic protein (MOBP), myelin-associated glycoprotein (MAG), plasmolipin, CD9) and enzymes (e.g. CNP)) was found (Cirelli et al., 2004).

Changes across the sleep/wake cycle are already known for the concentration of glutamate (Dash et al., 2009), a neurotransmitter shown to be involved in signalling from active neurons to oligodendrocytes, where it initiates MBP translation (Wake et al., 2011). Glutamate revealed increasing concentrations during wakefulness as well as rapid eye movement (REM) 
sleep and decreasing levels during non rapid eye movement (NREM) sleep (Dash et al., 2009). This fine-tuning of glutamate concentration is particularly important with respect to the energy metabolism of the brain, as it is known that a huge amount of the energy budget is needed to sustain synaptic activity at glutamatergic synapses (Attwell and Laughlin, 2001). This observation is consistent with the general lower energy demand of the brain during NREM sleep relative to waking and REM sleep (Madsen et al., 1991; Maquet, 1995) and contributes to homeostatic integration. If myelin gene expression levels are regarded as an indicator of the oligodendrocytic metabolism, both ATP and glutamate neurotransmitters might potentially mediate effects important for metabolic integration, as they have been shown to impact on myelination in an activity-dependent manner (Ishibashi et al., 2006; Kukley et al., 2007; Wake et al., 2011). Consistent with this idea, myelin genes (Cnp, Plp, $\mathrm{Mal}$ and $\mathrm{Mbp}$ ) in this study predominately revealed day time-dependent co-regulation.

\subsubsection{Region-dependent regulation}

There is evidence in the literature that glia may constitute a heterogeneous group of cells in the CNS, and this might contribute to the region-specific effects observed for myelin gene regulation. Astrocytes differ in morphology, developmental origin, gene expression profile, physiological properties, and function across brain regions and also within brain regions (Zhang and Barres, 2010). Several publications report huge differences in gene expression profiles when comparing astrocytes from various brain regions (Doyle et al., 2008; Yeh et al., 2009). Moreover, it is speculated that neuron-glia antigen 2 (NG2) glia/oligodendrocyte precursor cells possess a potential heterogeneity, as in vivo fate mapping analysis of NG2 cells by using different Cre transgenic driver lines (controlled by regulatory sequences of $P d g f \alpha, N g 2$, Olig2 or Plp genes) found that NG2 cells can develop into neurons, astrocytes and oligodendrocytes (Zhang and Barres, 2010). Moreover, Doyle and colleagues reported differences among cerebellar and cortical oligodendrocytes in mature and mixed populations. Generally, a huge diversity of translational profiles across neurons and glia was found providing novel insights into biochemical functions (Doyle et al., 2008). A region-specific heterogeneity of oligodendrocytes in relation to morphology has been known for a long time (Del Rio-Hortega, 1928), and more recent studies have reported that oligodendrocyte populations show differences in their expression profiles for neurotransmitter receptors (Takeda et al., 1995) and growth factors, such as brain-derived neurotrophic factor (BDNF) (Du et al., 2003), ciliary neurotrophic factor (CNTF) (Power et al., 2002), platelet-derived 
growth factor (PDGF)-AA, fibroblast growth factor (FGF)-2, and insulin-like growth factor (IGF)-1 (Mason and Goldman, 2002).

I speculate that my observations regarding the region-specific myelin gene expression could reflect different properties of oligodendrocytes (and potentially astrocytes). Both grey matter areas were largely consistent with myelin gene expression profiles; the white matter area, in contrast, displayed an altered expression pattern (inverse regulation in comparison to clock and activity markers) and also a reduction of amplitude. There is evidence in the literature that oligodendrocytes express neurotransmitter receptor subclasses for glutamate (N-methyl Daspartate (NMDA)- and $\alpha$-amino-3-hydroxy-5-methyl-4-isoxazolepropionic acid (AMPA)/kainate (KA)-type), glutamate transporters (Káradóttir and Attwell, 2007; Kolodziejczyk et al., 2010) and purinergic receptors (Fields and Burnstock, 2006). This might have an impact on myelination (Ishibashi et al., 2006; Kukley et al., 2007; Wake et al., 2011) (see above) by contributing to metabolic interactions between neurons and glia. Such a process could well differ between grey and white matter due to differences in receptor expression or spatial accessibility of neurotransmitters. Neurotransmitter concentrations are supposed to be high at synapses in grey matter, and might be lower in white matter due to more diffuse axonal release of glutamate (Kukley et al., 2007) and ATP (Fields and Ni, 2010). However, this hypothesis needs to be addressed in further studies.

Moreover, white and grey matter astrocytes differ in their glial fibrillary acidic protein (GFAP) expression, which is high in white matter (fibrous astrocytes) and low in grey matter (protoplasmic astrocytes). As astrocytes and GFAP have been implicated in activitydependent myelin gene expression via the secretion of leukemia inhibitory factor (LIF) (Ishibashi et al., 2006), white and grey matter astrocytes may exhibit differences in activitydependent signalling due to the variances in GFAP expression. In addition, specific kinase signalling cascades are differentially regulated in a circadian manner in white versus grey matter brain areas (Shahmoradi et al., unpublished data), and thus might also contribute to the observed region-specific gene regulation. Evidence supporting a region-specific regulation of myelin genes was given by a study by Swadlow and colleagues on rabbits showing that conduction velocity is plastic in $1 / 3$ of corpus callosal axons within one year after birth (Swadlow, 1985). However, despite increasing axonal length due to growing body size, CNS somatosensory and motor circuits reveal constant conduction delays after the first two years of age, pointing towards a compensatory mechanism that adapts conduction velocity in the CNS. Intriguingly, no compensatory mechanism was observed for the PNS, showing 
increasing conduction delays in proportion to length of the growing limb (Eyre et al., 1991). The attenuated regulation of myelin genes in the sciatic nerve might reflect this reduced level of plasticity. Accordingly, the same scenario might be true for the optic nerve. Therefore, I suggest that myelin genes contribute to a distinct form of plasticity (see above), which might be exclusively occurring in the brain. In this study, a micro-region of the ACC was chosen for analysis due to its importance in higher-order cognitive processing. For the isolation of the ACC and other brain subregions I employed laser-capture microdissection (LCM) which enables precise isolation of target micro-areas. Moreover, LCM ensured that information about the transcriptome is not lost due to dilution effects which occur when analyzing wider brain regions. Many studies implicate the ACC in controlling attention (Weissman et al., 2005), motivation and modulation of emotional aspects (Bush et al., 2000; Nieuwenhuis et al., 2001). Moreover, it has an important role in remote memory recall (Frankland and Bontempi, 2005; Goshen et al., 2011). Therefore, this particular brain region might need to display a high level of plasticity. The observed dynamic regulation of myelin genes in this study could well reflect this aspect. Moreover, plastic changes in myelin gene expression were observed also in SSMCX micro-area. This could be at least partly influenced by the enriched environment housing. Enriched environment is known to enhance motor, sensory and cognitive stimulation and therefore promotes neuronal activation, signalling and plasticity throughout various brain regions (Nithianantharajah and Hannan, 2006). Furthermore, the $\mathrm{CC}$ as the route for interhemispheric communication has also been shown to be plastic (see above). Correspondingly, we could show that myelin genes are dynamically regulated in the CC micro-region, however, the profile differed from the cortical micro-regions. Due to these highly region-specific effects of myelin gene expression which potentially reflects complex higher-order cognitive processes, it was crucial to analyse precisely defined cortical areas. However, to address the question if modulation of environmental conditions would influence myelin plasticity in certain brain regions, further experiments combining corresponding housing with precise isolation of brain areas of interest would be necessary.

\subsection{Attenuated expression of myelin genes in $\mathrm{S} 1 / 2^{-/-}$mice}

The dynamic regulation of myelin gene expression observed in WT mice was attenuated in $\mathrm{S} 1 / 2^{-/-}$animals in both cortical and the corpus callosum micro-area. Per2 expression displayed no obvious differences between genotypes, indicating a functional core clock, which is in concordance with published data (Bode et al., 2011; Rossner et al., 2008). Fos regulation was 
also comparable between WT and mutant mice in grey matter micro-areas. In the CC microarea the regulation was lower than in grey matter areas in both genotypes, potentially due to the lack of neurons. A mild peak of Fos already occurred at ZT4.

In the sciatic nerve, Per2 kinetics were shifted to earlier time points in both genotypes, potentially due to the increased responsiveness of the sciatic nerve to anticipatory motor activity (see above). This effect was further enhanced in $\mathrm{S} 1 / 2^{-/-}$mice, potentially caused by the attenuated sleep-wake biology of Sharp mutant mice (Shahmoradi et al., in submission) (see above). Consistently, Fos expression, indicating neuronal activity, was also shifted to earlier time points in the sciatic nerve kinetic analysis.

\subsubsection{Regulation of myelin genes in response to sleep deprivation}

SHARP1 and -2 proteins have been shown to be involved in the homeostatic regulation of sleep (Shahmoradi et al., in submission). Therefore, myelin gene expression was further analysed upon sleep deprivation (SD). We focused on the cortex because of its fundamental role in regulating sleep and its effects upon SD (Borbély and Achermann, 1999; Van Dongen et al., 2003; Horne, 1988; Steriade and Hobson, 1976). As it was already known that the relative amount of rapid eye movement (REM) sleep was changed in mutant mice upon total SD by gentle handling (Shahmoradi et al., in submission), the inverted flowerpot technique of SD was applied, known to particularly prevent REM sleep (Cohen and Dement, 1965; JOUVET et al., 1964).

WT mice showed a clear response to SD at the molecular level as both Per2 and Fos gene expression was down-regulated, whereas these effects were attenuated in $\mathrm{S} 1 / 2^{-/-}$mice. According to the literature, Per2 is influenced both by the time of day and also by activity in rats and mice (Cirelli et al., 2004; Masubuchi et al., 2000; Wisor et al., 2002). Upon SD applied by gentle handling for eight hours, Cirelli and colleagues described an induction of Per2 transcription in the rat cerebral cortex when compared to the undisturbed sleeping control group (Cirelli et al., 2004). This also could be shown for mice that were sleep deprived by introducing novel objects (Wisor et al., 2002). Moreover, the expression of the IEG Fos was also enhanced upon loss of sleep (Cirelli et al., 1995, 2006; Terao et al., 2006). In this thesis, the observed opposite effects of Per 2 and Fos regulation might be influenced by the inverted flowerpot method. This technique induces a certain amount of stress for the mice which are placed on small cylinders (inverted flowerpots) in a special cage filled with water (see Fig. 17). Moreover, for the time of sleep deprivation, mice are also food deprived. 
However, the attenuated IEG response in mutant mice is consistent with the reduced activitydependent Fos expression in the cortex (Shahmoradi et al., in submission). In WT mice, Cnp and $M b p$ expression were markedly reduced upon SD, whereas these effects were attenuated in the mutant mice. This is consistent with the observed reduced dynamic regulation in the micro-areas. The regulation for Plp and Mal was different, as in WT mice both genes were induced upon loss of sleep, whereas the opposite was the case for mutant mice. Apparently, different myelin genes respond differentially to the SD paradigm. From the literature it is already known that at least some myelin genes were regulated upon SD, as this was shown for Cnp2, Plp and Mog (Cirelli et al., 2006). However, the regulation seemed to be complex and might be highly dependent on the SD method and duration (short term or long term) (Cirelli et al., 2006).

\subsubsection{Consequences with respect to the metabolism}

I hypothesize that the attenuated regulation of myelin genes in $\mathrm{S} 1 / 2^{-/-}$mice indicates the uncoupling of metabolic integration from myelination. Interestingly, Sharp2 expression is connected to neuronal activity induced by kainic acid in vivo (Rossner et al., 1997). Therefore, SHARP proteins could act as integration factors, which are involved in the metabolic coupling of oligodendrocytes to neuronal activity to maintain energy homeostasis. Both transcription factors may exert repressive and co-activating functions depending on the circadian time and/or behavioural state to integrate myelin gene expression. Disintegration of tightly regulated processes could potentially affect the entire organism's physiology and could have metabolic consequences. Of note, mutant mice show signs of premature aging such as the early appearance of cataracts, grey hair and the development of tumours, accompanied by a reduced lifespan (Shahmoradi et al., unpublished data). The observed hypoactivity and the fragmented sleep-wake episodes could also represent indicators of a disturbed metabolism and may pinpoint towards an increased need for rest. Other clock genes have been described to play a role in the metabolism. Clock mutant mice are obese and show a metabolic syndrome including hyperlipidemia, hyperglycaemia and hypoinsulinemia (Rudic et al., 2004; Turek et al., 2005). The deletion of Bmall in mice leads to a defective glucose homeostasis (Rudic et al., 2004), premature aging and reduced lifespans (Kondratov et al., 2006). Also, Per2 mutant mice display alterations in the glutamatergic system associated with an enhanced voluntary alcohol consumption (Spanagel et al., 2005) and increased tumour formation (Fu et al., 2002). However, further studies need to be conducted to elucidate whether the effects described here 
for SHARP1 and -2 depend on their function connected to the core molecular clock, or whether they exert roles independent role of core clock genes.

\subsubsection{Consequences with respect to psychiatric disorders}

The attenuated effects seen for myelin gene expression in $\mathrm{S} 1 / 2^{-/-}$mice might be of importance for psychiatric diseases. It is known that myelin gene expression is altered in schizophrenia, depression and bipolar disorder (Aston et al., 2005; Georgieva et al., 2006; Tkachev et al., 2003), potentially resulting from a dis-connectivity of neuronal circuits ('dis-connection hypothesis' by Friston (Friston, 1999)), which might contribute to cognitive dysfunction being one of the endophenotypes of schizophrenia (Gottesman, 2003). Behavioural alterations in $\mathrm{S} 1 / 2^{-/-}$mice together with a reduction of day time-dependent changes in cortical gene expression and correlations with human data sets showed prominent associations for several endophenotypes of psychiatric disorders (Shahmoradi et al., in submission). The attenuated expression of myelin genes further supports the hypothesis that $\mathrm{S} 1 / 2^{-/-}$mice display symptoms reminiscent of psychiatric disorders. Therefore, these mice constitute a promising model to study the development of psychiatric diseases, which could well complement post mortem analysis of human material. Hypothetically, the disruption of dynamic myelin gene expression, as shown for $\mathrm{S} 1 / 2^{-/-}$mice, could be at the onset of disease development. In addition, gene expression analysis in the context of psychiatric disorders for the first time was dedicated to precise micro-regions in the cortex and corpus callosum, whereas previous studies focused on larger cortical areas.

\subsubsection{Reduced expression levels of myelin genes in primary cultured oligodendrocytes from $\mathrm{S} 1 / 2^{-/-}$mice}

Differentiated, MBP-positive oligodendrocytes were stimulated with neuronal conditioned medium (NCM) mimicking neuronal input to analyse the influence on myelin gene regulation and potential genotype-dependent differences. WT and mutant cultures were comparable in terms of cell numbers of MBP-stained oligodendrocytes. However, numbers of GFAPpositive cells were almost twice as high in mutant cultures, an effect that was already observed in vivo in the cortex of mutant mice (Rossner et al., unpublished observation). Myelin gene expression analysis revealed a prominent down-regulation of $C n p, P l p$ and $M b p$ in mutant cultures. Western blotting showed that these changes on RNA level were accompanied by a reduction in myelin protein expression. Weaker, but still clear effects could 
be shown for genes involved in lipid metabolism, which also were down-regulated in mutant cultures. Although it is difficult to interpret the subtle variability in kinetic expression, similar patterns for Fos, Cnp (only WT), Mbp (only WT), Hmgcr, ApoA1, as well as Sharpl and -2 could be observed, all indicating a slight increase of expression eight hours after NCM administration. Of note, Gfap expression was elevated in mutant cultures, which corresponded to histological data already observed in the mutant cortex (see above). Increased GFAP expression is known to be a marker for activated astrocytes (Eng and Ghirnikar, 1994). S100b expression, however, considered to be a pan-astrocytic marker, was higher in WT cultures, indicating that the elevated Gfap signal in mutant cultures does not necessarily correlate with an increased number of astrocytes.

For the kinetic analysis, gene expression was additionally monitored at later time points to ensure a compete data set. The prominent down-regulation of Cnp, Plp and Mbp in mutant cultures was confirmed in an independent experiment. In agreement with the first kinetic analysis, genes implicated in lipid metabolism also showed reduced expression levels in mutant cultures. Directly after NCM application, myelin genes were induced in WT cultures, whereas this effect could not be seen in mutant cells. Intriguingly, also Fos expression showed these genotype-specific effects. The lack of SHARP proteins as homeostatic integration factors and potential mediators of activity-dependent gene expression could result in these attenuated effects observed in mutant cultures. However, at later time points myelin gene expression was higher upon NCM administration in cultures from both genotypes when compared to the non-stimulated control. The second kinetic experiment revealed enhanced myelin expression upon NCM stimulation, which is consistent with both the literature (Bologa et al., 1986) and my current model regarding neuronal input (as mimicked by NCM application), wherein stimulating effects are exerted on myelin genes. Again, myelin gene expression behaved similar in WT and mutant cultures. Mbp showed a second peak of expression which was also observed for Hmgcr and S100b. Sharpl and -2 also displayed coregulation. In this setup, high expression of latter genes correlated with a trough of myelin gene regulation (at 24 hours after NCM application).

The reduced expression of myelin genes and proteins in primary cultured oligodendrocytes from S1/2 $2^{-/-}$mice are of high interest in the context of a report from the Barres lab. The authors demonstrated that at least Sharpl mRNA was up-regulated at later stages of oligodendrocyte differentiation in vitro (Dugas et al., 2006), potentially paralleling myelin gene expression. I speculate that the SHARP proteins could be important factors in 
modulating myelin gene expression by a so far unknown mechanism. As already mentioned, Sharp 2 mRNA was shown to be up-regulated in response to hyperactivity induced by kainic acid in rats (Rossner et al., 1997), which might support the role of SHARP proteins in mediating activity-dependent cellular changes.

As the in vivo data indicated an attenuated regulation of myelin genes rather than a reduction in expression, I hypothesize that mutant oligodendrocyte cultures may exhibit a delay in myelin gene expression due to the lack of adaptation factors. It would be interesting to analyse if this delay could be rescued in a co-culture system with neurons and astrocytes. Moreover, a developmental analysis of WT and mutant mice during early postnatal and also adult stages on mRNA and protein level would clarify if there is also a delay in myelination in vivo.

\subsection{Mechanistic insight}

Sleep/wake behaviour, running wheel activity and cortical gene expression between day and night were attenuated in $\mathrm{S} 1 / 2^{--}$mice, although the total amount of sleep and wakefulness and the circadian rhythm were unchanged (Shahmoradi et al., in submission). Therefore, SHARP1 and -2 likely act as important integration factors of the circadian clock and in sleep homeostasis. The attenuated effects in myelin gene regulation support role of SHARP proteins in homeostatic/metabolic integration. In the double mutant mice, homeostatic integration is altered, potentially resulting in a deregulation of various genes, as shown here for myelin genes. However, the underlying mechanisms are still elusive. In this study, SHARP1 and -2 have been shown to exert repressive and co-activating functions in a contextspecific manner in the circadian clock. The repressive function was dependent on the basic domain, which mediates DNA-binding to E-box elements in the promoter regions of Clock genes. The co-activating function was associated with the presence of interaction partners for SHARP proteins, such as BMAL1.

Apart from their role in the circadian clock, SHARP1 and -2 have been described to serve a variety of functions by exerting either inhibitory or activatory actions, dependent on the promoter of the target genes and the tissue. For instance, SHARP1 is a potent transcriptional repressor of MyoD- and E12-mediated differentiation by direct binding to these bHLH proteins, and by competition for E-Box elements (Azmi et al., 2003). Moreover, SHARP2 inhibits adipocyte differentiation by repressing the nuclear receptor peroxisome proliferatoractivated receptor gamma (PPAR $\gamma 2$ ) promoter activation (Yun et al., 2002). SHARP1 
physically binds to the signal transducer and activator of transcription 3 (STAT3), and was also shown to be a transcriptional activator from STAT3-dependent cis-elements (Ivanova et al., 2004). STAT3 plays a key role in cell growth and apoptosis. Reporter gene assays indicated repressive effects of SHARP1 and -2 on retinoic $\mathrm{X}$ receptor (RXR) and liver $\mathrm{X}$ receptor (LXR) transactivation. RXR and LXR are metabolite-sensing nuclear receptors (ligand-inducible transcription factors) and many of them have been shown to interact with clock genes (Teboul et al., 2008), which might provide a link between clock and metabolism (Cho et al., 2009). Interestingly, RXR $\gamma$ signalling was shown to activate re-myelination in the CNS (Huang et al., 2011). SHARP2 is also reported to bind to SP1 sites (Li et al., 2006), a fact that could be of particular importance, as there are at least three SP1 sites described in the Mal promoter (Tugores et al., 1997).

To gain mechanistic insight with respect to myelin gene regulation, the function of SHARP proteins on the $M a l$ and $M b p$ promoter activity was addressed in luciferase reporter gene assays. The oligodendroglial precursor cell line Oli-neu (Jung et al., 1995) was used to generate cell lines stably expressing the firefly luciferase under control of either the Mal or the $M b p$ proximal promoter region. Reporter gene assays upon infection with a SHARP2 expressing lentivirus indicated an activating effect for SHARP2 at least on the Mal promoter. Notably, reporter gene assays in primary cultured oligodendrocytes showed that SHARP1 and -2 double transfection significantly increased Mal promoter activity. These results are very interesting with respect to the in vivo and in vitro data, suggesting that in the knockout situation activating (and potentially context-dependent inhibitory) effects of SHARP proteins lead to an attenuated myelin gene amplitude (in vivo) or to reduced expression levels (in vitro). However, the mechanism is still elusive. Promoter analysis using the TRANScription FACTor database (TRANSFAC; http://transfac.bioinf.med.uni-goettingen.de), a public database for predicting transcription factor binding sites, indicated multiple binding sites for RXRs and SP1 in the Mal and Mbp promoter regions. Moreover, E-box sequences were found representing putative binding sites for E12, E47 and MyoD. Of note, two potential binding sites, i.e. E-box sequences, for SHARP1 (CAACTG) have been identified in the Mal promoter, and one putative SHARP1 binding site (CACTTGA) is also present in the Mbp promoter. These potential interaction sites might contribute to the SHARP-dependent effects on myelin promoter activation described above. Further analysis will elucidate the precise mechanisms. 


\subsection{Concluding remarks}

In the present thesis, behavioural analyses of $\mathrm{S} 1 / 2^{-/-}$mice revealed phenotypes reminiscent of mixed-state criteria towards bipolar disorder which provides further evidence that this mouse model is of high interest with respect to psychiatric disorders. I also could show for the first time that myelin genes display an unexpected high degree of plasticity on mRNA level. Precise micro-regions in the cortex and corpus callosum displayed a region-specific dynamic expression of major myelin genes that might be coupled to neuronal activity and/or the circadian system. This finding is of particular interest as adult plasticity of white matter responds to learning, thereby potentially contributing to enhanced information processing among distant brain regions. Moreover, this dynamic myelin gene expression was attenuated in $\mathrm{S} 1 / 2^{-/-}$mice, a mouse model lacking two bHLH transcription factors supposed to play a role as adaptation factors for the circadian system and to be involved in homeostatic control of sleep. This particular finding could attract more attention on the $\mathrm{S} 1 / 2^{-/-}$mouse model, since it is known that myelin genes are deregulated in psychiatric diseases such as bipolar disorder, schizophrenia and depression. In mutant mice, myelin gene regulation was also altered upon sleep deprivation, supporting the hypothesis that SHARP1 and -2 exert important functions in metabolic integration. Moreover, primary cultured oligodendrocytes from mutant mice showed a reduced expression of myelin genes and proteins. Consistent with this, reporter gene assays in Oli-neu cells and primary cultured oligodendrocytes indicated an activating role of SHARP transcription factors on myelin promoters. Thus, SHARP proteins may be a crucial nodal point in the integration of metabolic and myelination-dependent processes. 


\section{Material}

\subsection{Chemicals and reagents}

Standard chemicals, which are not listed separately were obtained from commercial companies like Amersham Biosciences, BD Falcon, BioRad, Carl Roth, Eppendorf, Invitrogen, Merck, Riedel de Haen, Roche, Serva, Sigma or VWR.

\begin{tabular}{|c|c|}
\hline Agarose & Biozym, Seakem LE Agarose \\
\hline Ampicillin & Sigma-Aldrich \\
\hline Aqua Poly Mount (mounting medium) & Polyscience Ltd. \\
\hline ATP & PJK \\
\hline BSA & Sigma-Aldrich \\
\hline Coenzyme A & PJK \\
\hline Chloramphenicol & Sigma-Aldrich \\
\hline Chloroform & Sigma-Aldrich \\
\hline DAB & Dako Cytomation \\
\hline DAPI & Roche \\
\hline DNA ladder (100 bp, $1 \mathrm{~kb})$ & Fermentas \\
\hline DMEM (Dulbecco`s Modified Eagle Medium) & Gibco \\
\hline DTT & PJK \\
\hline EDTA & Sigma-Aldrich \\
\hline Ethidiumbromide & Sigma-Aldrich \\
\hline Eukitt (mounting medium) & Kindler GmbH \\
\hline FCS (fetal calf serum) & Gibco \\
\hline Glycerin & Sigma-Aldrich \\
\hline Hemalaun & Merck \\
\hline Hoechst 33342 & Molecular Probes \\
\hline HS (horse serum) & Gibco \\
\hline Kanamycin & Sigma-Aldrich \\
\hline D-luciferin & PJK \\
\hline Non fat milk powder & Töpfer \\
\hline OptiMEM & Gibco \\
\hline Paraformaldehyde & Serva \\
\hline PEI (polyethylenimine) & Polysciences \\
\hline Penicillin/Streptavidin & Sigma-Aldrich \\
\hline Poly-L-lysin & Sigma-Aldrich \\
\hline 2-Propanol & VWR \\
\hline Protease-inhibitor tablets (Complete) & Roche \\
\hline Sterile filters $(0.45 \mu \mathrm{m})$ & Sartorius \\
\hline SDS & Sigma \\
\hline Trypsin & Gibco \\
\hline
\end{tabular}




\subsection{Laboratory supplies}

Consumable supplies which are not mentioned separately were purchased from commercial companies like BD Falcon, Eppendorf, Gilson, Greiner-Nunc and Menzel-Gläser.

CapSure Macro LCM caps

Cell culture dishes

Cryo tubes

ECL-hyperfilms

Electroporation cuvettes $(0.1$ and $0.4 \mathrm{~mm})$

Glass slides, cover slips

NuPAGE 4-12\% Bis-Tris Gels

PEN Membrane Frame Slides

PVDF Membrane Hybond P

Reaction tubes

Ultra-15 Centrifugal Filter Unit

96-well plates for qRT-PCR
Arcturus

BD Falcon

Nunc

Amersham Biosciences

Biorad

Marienfeld

Invitrogen

Molecular devices

Amersham Biosciences

Eppendorf

Millipore

Applied Biosystems

\subsection{Laboratory equipment}

Below only special equipment is listed that extends standard laboratory equipment.

7500 Fast Real-Time-PCR System

Arium 611 Water Purification System

Computer hardware

Canon iRC3580

HP Color Laser Jet 4550

MacBook Pro

OKI C9200

Computer-Software

7500 Fast System SDS software

Acrobat Reader 8 Professional

DNASTAR Lasergene 8

Fiji

Firefox web browser

GraphPad Prism 4

Illustrator CS3

InDesign CS3

Microsoft Office X

Photoshop CS3

Universal Probe Library Assay Design Center

Zotero

Cryostate Cryocut CM3000

Digital camera ProgRes C14

Embedding centre AP 280

GenePulser Xcell
Applied Biosystems

Sartorius

Canon

Hewlett Packard

Apple

OKI Systems

Applied Biosystems

Adobe

DNAStar

http://fiji.sc/wiki/index.php/Downloads

Mozilla Foundation

www.graphpad.com

Adobe

Adobe

Microsoft

Adobe

https://www.roche-applied-

science.com/sis/rtpcr/upl/adc.jsp

http://www.zotero.org/

Leica

Jenaoptik

Mikrom

Biorad 
Hood

Laser-Microdissection instrument

Microplate reader Mithras LB 940

Microscopes

Leica DM IRBE (invers)

Leica DM RXA

Leica TCS SP5 X (confocal)

Nucleofector

Open field system

SDS-PAGE gel electrophoresis and

Xcell SureLock NuPAGE system

Sliding microtome HM 400

Sonicator Bandelin Sonoplus

Tissue processor HMP 110

UV-illuminator

X-ray film developer KODAK XOMAT

\subsection{Kits}

DC Protein Assay

ECL Plus Western-Blot Detection Reagents

pGemT Vector System I

Mouse Neuron Nucleofector Kit

NucleoSpin Plasmid Quick Pure Mini Kit

NucleoBond Xtra Midi EF Kit

NucleoSpin Gel and PCR Clean-up Kit

RNeasy Micro Kit

RNeasy Mini Kit

Power SYBR Green Master Mix (2x)

TaqMan Universal PCR Master Mix

Transcriptor High Fidelity cDNA Synthesis Kit
Heraeus

Veritas

Berthold Technologies

Leica

Amaxa

TSE Systems

Invitrogen

Mikrom

Bandelin

Mikrom

Intas UV-Systems

Kodak

Bio-Rad

Amersham Biosciences

Promega

Amaxa

Macherey-Nagel

Macherey-Nagel

Macherey-Nagel

Qiagen

Qiagen

Applied Biosystems

Applied Biosystems

Roche

\subsection{Enzymes}

Calf intestine phosphatase (CIP)

Cloned $P f u$ Ultra DNA polymerase

Roche

GoTaq DNA polymerase

Stratagene

HotStarTaq DNA polymerase

Sigma-Aldrich

Qiagen

Easy-A HiFi PCR cloning enzyme

T4 DNA-ligase

Stratagene

DNAseI

BP clonase II

Promega

Promega, Roche

Invitrogen

LR clonase II

Invitrogen

LR clonase II Plus

Invitrogen

Proteinase K

Invitrogen

Restriction enzymes

SuperscriptIII Reverse Transcriptase

New England Biolabs

Invitrogen 


\subsection{Antibodies}

\subsubsection{Primary antibodies}

$\alpha$-CNP, monoclonal mouse $\alpha$-GFAP, monoclonal mouse $\alpha$-MAL, polyclonal rabbit

$\alpha$-MBP, polyclonal rabbit

$\alpha$-OLIG2, polyclonal rabbit

$\alpha$-PLP, polyclonal rabbit

$\alpha-\alpha$-Tubulin, monoclonal mouse
Sigma-Aldrich

Novocastra

Prof. Schaeren-Wiemers,

Department of Biomedicine, Basel

Chemicon

Prof. Stiles, Department of

Neuroscience, Harvard

Department of Neurogenetics,

MPI, Goettingen

Sigma-Aldrich

\subsubsection{Secondary antibodies}

HRP-conjugated-goat- $\alpha$-mouse

Dianova

HRP-conjugated-goat- $\alpha$-rabbit

Dianova

Cy2-conjugated-goat- $\alpha$-mouse

Dianova

Cy3-conjugated-goat- $\alpha$-rabbit

Dianova

\subsection{Oligonucleotides}

Oligonucleotides were synthesized at the Institute's DNA Core Facility (Department of Neurobiology, MPI of Experimental Medicine, Goettingen) lead by Dr. Fritz Benseler. The house identification numbers are displayed.

\subsubsection{Oligonucleotides for genotyping of SHARP1 and -2 null mutant mice}

\begin{tabular}{|l|l|l|c|}
\hline \multicolumn{2}{|c|}{ Primer } & \multicolumn{1}{|c|}{ 5'- 3' Sequence } & Number \\
\hline \multirow{3}{*}{ SHARP1 } & SHARP1 Exon 3 2 fwd & ACCTACAAGTTACCGCACAG & 6275 \\
\cline { 2 - 4 } & SHARP1 2 rev & TTTCTCCAAATGCCCCAGTG & 6274 \\
\cline { 2 - 4 } & KO-2 Neo 6 rev & GCAATCCATCTTGTTCAATGGC & 2382 \\
\hline \multirow{3}{*}{ SHARP2 } & SHARP2 Neo pos. 611 fwd & TGGGCTGACCGCCTCGTGC & 10168 \\
\cline { 2 - 4 } & SHARP2 fwd & GGAAGCTCAGGCTAGCTCAT & 1970 \\
\cline { 2 - 4 } & SHARP2 rev & CGTTTTATTCCCCGCCTGGA & 1971 \\
\hline
\end{tabular}




\subsubsection{Oligonucleotides for cloning}

Deletion of the basic (b) region of pEXPR 3xFLAG SHARP1 and pEXPR HA SHARP2 plasmids

\begin{tabular}{|c|c|c|c|}
\hline \multicolumn{2}{|r|}{ Primer } & 5'-3' Sequence & Number \\
\hline \multirow{7}{*}{ SHARP $1^{\wedge} b$} & $\begin{array}{l}\text { Deletion of } b \text { region for fusion } \\
\text { PCR fwd }\end{array}$ & $\begin{array}{l}\text { CTCTTTGTATATGTGTAAACCCAAAAGGAGCTTGAA } \\
\text { GCGAGACGATACCAAG }\end{array}$ & 11718 \\
\hline & $\begin{array}{l}\text { Deletion of } \mathrm{b} \text { region for fusion } \\
\text { PCR rev }\end{array}$ & $\begin{array}{l}\text { CAGCTGAGCAATGCATTCATTAATTCGGTCCTTGGT } \\
\text { ATCGTCTCGCTTC }\end{array}$ & 11719 \\
\hline & $\begin{array}{l}\text { Primer for fusion PCR final } \\
\text { fwd }\end{array}$ & CATCGATTACAAGGATGACGATGACAAGCTT & 11720 \\
\hline & Primer for fusion PCR final rev & GTCGTGTTCCGGCTCCGTG & 11721 \\
\hline & $\begin{array}{l}\text { Deletion of b region fragment } 1 \\
\text { primer fusion PCR } 1 \text { fwd }\end{array}$ & GACCGAATTAATGAATGCATTGCTCAGCTG & 11841 \\
\hline & $\begin{array}{l}\text { Deletion of b region fragment } 1 \\
\text { primer fusion PCR } 1 \text { rev }\end{array}$ & $\begin{array}{l}\text { CTTGGTATCGTCTCGCTTCAAGCTCCTTTTGGGTTTA } \\
\text { CACATATACAAAGAG }\end{array}$ & 11840 \\
\hline & $\begin{array}{l}\text { Deletion of b region fragment } 2 \\
\text { primer fusion PCR } 2 \mathrm{fwd}\end{array}$ & $\begin{array}{l}\text { GAAGCGAGACGATACCAAGGACCGAATTAATGAAT } \\
\text { GCATTGCTCAGCTG }\end{array}$ & 11842 \\
\hline \multirow{7}{*}{$\mathrm{SHARP} 2^{\wedge} \mathrm{b}$} & $\begin{array}{l}\text { Deletion of } b \text { region for fusion } \\
\text { PCR fwd }\end{array}$ & $\begin{array}{l}\text { GTACAAGTCCAGGCGGGGAATAAAACGGAGCGAGG } \\
\text { ACAGCAAG }\end{array}$ & 11722 \\
\hline & $\begin{array}{l}\text { Deletion of } \mathrm{b} \text { region for fusion } \\
\text { PCR rev }\end{array}$ & $\begin{array}{l}\text { GGCAATGCACTCGTTAATCCGGTCCTTGCTGTCCTC } \\
\text { GCTC }\end{array}$ & 11723 \\
\hline & $\begin{array}{l}\text { Primer for fusion PCR final } \\
\text { fwd }\end{array}$ & GGGCTACCCCTACGACGTGC & 11724 \\
\hline & Primer for fusion PCR final rev & CAGGGCCATGATTTTCTGCTGCTG & 11725 \\
\hline & $\begin{array}{l}\text { Deletion of } \mathrm{b} \text { region fragment } 1 \\
\text { primer fusion PCR } 1 \mathrm{fwd}\end{array}$ & GACCGGATTAACGAGTGCATTGCC & 11844 \\
\hline & $\begin{array}{l}\text { Deletion of } \mathrm{b} \text { region fragment } 1 \\
\text { primer fusion PCR } 1 \mathrm{rev}\end{array}$ & $\begin{array}{l}\text { CTTGCTGTCCTCGCTCCGTTTTATTCCCCGCCTGGAC } \\
\text { TTGTAC }\end{array}$ & 11843 \\
\hline & $\begin{array}{l}\text { Deletion of b region fragment } 2 \\
\text { primer fusion PCR } 2 \text { fwd }\end{array}$ & $\begin{array}{l}\text { GAGCGAGGACAGCAAGGACCGGATTAACGAGTGCA } \\
\text { TTGCCCAGCTGAAG }\end{array}$ & 11845 \\
\hline
\end{tabular}

Cloning of Mal and $M b p$ promoter regions by adding B1 and B4 sites for multisite gateway

\begin{tabular}{|c|c|c|c|}
\hline \multicolumn{2}{|c|}{ Primer } & 5'- 3' Sequence & Number \\
\hline \multirow{4}{*}{$\begin{array}{l}\text { Mal } \\
\text { promoter }\end{array}$} & Primer fwd & CATTGTAGAGTCAACAGCACCTTTGTGG & 16508 \\
\hline & Primer rev & GCCTGCGAGCTGGCGTCG & 16509 \\
\hline & $\begin{array}{l}\text { B1 primer } \\
\text { fwd }\end{array}$ & $\begin{array}{l}\text { GGGGACAAGTTTGTACAAAAAAGCAGGCTCTCATTGTAGAGTCAACA } \\
\text { GCACCTTTGTGG }\end{array}$ & 16511 \\
\hline & $\begin{array}{l}\text { B4 primer } \\
\text { rev }\end{array}$ & GGGGCAACTTTGTATAGAAAAGTTGGCCTGCGAGCTGGCGTCG & 16512 \\
\hline \multirow{4}{*}{$\begin{array}{l}\text { Mbp } \\
\text { promoter }\end{array}$} & Primer fwd & AAGCTTTGAGAGAAAAGGGACCAGATCTTATTCC & 16828 \\
\hline & Primer rev & CCCGGAAGCTGCTGTGGGGT & 16829 \\
\hline & $\begin{array}{l}\text { B1 primer } \\
\text { fwd }\end{array}$ & $\begin{array}{l}\text { GGGGACAAGTTTGTACAAAAAAGCAGGCTCTAAGCTTTGAGAGAAAA } \\
\text { GGGACCAGATCTTATTCC }\end{array}$ & 16830 \\
\hline & $\begin{array}{l}\text { B4 primer } \\
\text { rev }\end{array}$ & GGGGCAACTTTGTATAGAAAAGTTGCCCGGAAGCTGCTGTGGGGT & 16831 \\
\hline
\end{tabular}

\subsubsection{Oligonucleotides for Real-Time-PCR}

\begin{tabular}{|l|l|l|l|}
\hline \multicolumn{1}{|c|}{ Primer } & \multicolumn{1}{|c|}{ Orientation } & \multicolumn{1}{c|}{$\mathbf{5}$ '- 3' Sequence } & Number \\
\hline $\begin{array}{l}\text { B-Actin } \\
\text { TaqMan }\end{array}$ & Fwd & ACGGCCAGGTCATCACTATTG & 11280 \\
\cline { 2 - 4 } ApoA1 & Rev & AGGAAGGCTGGAAAAGAGCC & 11281 \\
\cline { 2 - 4 } & Fwd & GGACACTCTGGGTTCAACCG & 4336 \\
\cline { 2 - 4 } $\begin{array}{l}\text { Atp5b } \\
\text { TaqMan }\end{array}$ & Fwd & TCCCAGAAGTCCCGAGTCAA & 4337 \\
\cline { 2 - 4 } BlastR & Rev & CTATCTGCTGGCCCCATAC & 11282 \\
\cline { 2 - 4 } & Fwd & CATTGAAAGAGCAACGGCTA & 21283 \\
\hline Cnp & Fwd & TGGCGACGCTGTAGTCTTC & 22945 \\
\hline
\end{tabular}




\begin{tabular}{|c|c|c|c|}
\hline TaqMan & Rev & GTCCCTAGCATGTGGCAGCT & 11285 \\
\hline \multirow{2}{*}{ Cyc1 } & Fwd & CAGAGCATGACCATCGAAAA & 10572 \\
\hline & Rev & CACTTATGCCGCTTCATGG & 10573 \\
\hline \multirow{2}{*}{ Fos } & Fwd & GAATGGTGAAGACCGTGTCA & 8879 \\
\hline & Rev & TCTTCCTCTTCAGGAGATAGCTG & 8892 \\
\hline \multirow{2}{*}{ Gfap } & Fwd & ACAGACTTTCTCCAACCTCCAG & 11596 \\
\hline & Rev & CCTTCTGACACGGATTTGGT & 11597 \\
\hline \multirow{2}{*}{ Hmgcr } & Fwd & TGAAGAGGACGTGCTGAGCA & 4336 \\
\hline & Rev & ATGTACAGGATGGCGATGCA & 4337 \\
\hline \multirow{2}{*}{$\begin{array}{l}\text { Mal } \\
\text { TaqMan }\end{array}$} & Fwd & GGTGAGATCGAAATGTGCCC & 11290 \\
\hline & Rev & CCGCTGGGTGTGTTAATGTG & 11291 \\
\hline \multirow[t]{2}{*}{$\mathrm{Mbp}$} & Fwd & GCCTGTCCCTCAGCAGATT & 8679 \\
\hline & Rev & GCCTCCGTAGCCAAATCC & 8678 \\
\hline \multirow[t]{2}{*}{ Per2 } & Fwd & CAACACAGACGACAGCATCA & 8738 \\
\hline & Rev & TCCTGGTCCTCCTTCAACAC & 8739 \\
\hline \multirow{2}{*}{$\begin{array}{l}\text { Plp } \\
\text { TaqMan }\end{array}$} & Fwd & GGCTAGGACATCCCGACAAGT & 11292 \\
\hline & Rev & GGCAAACACCAGGAGCCATA & 11293 \\
\hline \multirow{2}{*}{ S100b } & Fwd & AACAACGAGCTCTCTCACTTCC & 12033 \\
\hline & Rev & CTCCATCACTTTGTCCACCA & 12034 \\
\hline \multirow{2}{*}{$\begin{array}{l}\text { Sharp1 } \\
\text { TaqMan }\end{array}$} & Fwd & AGCGCTCTCTGAAATCGCC & 11294 \\
\hline & Rev & GCAGGTTTGAAACCCCGAG & 11295 \\
\hline \multirow{2}{*}{ Sharp2 } & Fwd & TCTCCTACCCGAACATCTCAA & 8690 \\
\hline & Rev & AATGCTTTCACGTGCTTCAA & 8691 \\
\hline \multirow{2}{*}{ Sqs } & Fwd & TCAATCAGACCAGTCGCAGC & 4350 \\
\hline & Rev & GTGCCGTATGTCCCCATCC & 4351 \\
\hline \multirow{2}{*}{ Top1 } & Fwd & AATGAGAGGGGACAGCAAAC & 10576 \\
\hline & Rev & CCTTGTCTGTGGCCTTCG & 10577 \\
\hline
\end{tabular}

\title{
6.7.4 Oligonucleotides for cDNA synthesis
}

\begin{tabular}{|l|l|l|}
\hline \multicolumn{1}{|c|}{ Primer } & \multicolumn{1}{|c|}{ 5'-3' Sequence } & \multicolumn{1}{c|}{ Number } \\
\hline Oligo dT anchor primer & TTTTTTTTTTCTTTTTTTTTTTTCC & 9578 \\
\hline Random 9mer & NNNNNNNNN & 4542 \\
\hline Random 15mer & NNNNNNNNNNNNNNN & 10596 \\
\hline
\end{tabular}

\subsection{Plasmids}

\subsubsection{For reporter gene assays}

\author{
pEXPR 3x FLAG mSHARP1 \\ pEXPR HA rSHARP2 \\ pEXPR 3x FLAG mSHARP1^b \\ pEXPR HA rSHARP2 $2^{\wedge} b$ \\ pEXPR HA NPAS2 \\ pEXPR HA BMAL1 \\ pCDNA3 \\ pEYFPnuc \\ pGL2 9xE-Box TK promoter \\ phRLuc/SV40 \\ phRLuc/TK \\ phRLuc/CMV
}




\title{
6.8.2 For stable cell lines
}

pEXPR Mal prom EXT (GL4)

pEXPR Mbp prom EXT (GL4)

\subsubsection{For virus experiments}

pLenti6 SHARP2

pLenti6 EYFP

pLenti6 EXPR

psPAX2 (viral packaging plasmid)

pMD2.G (viral envelope plasmid)

\subsection{Bacterial strains}

\author{
E. coli $\mathrm{DH} 5 \alpha$ \\ E. coli ElectroMAX DH10B \\ E. coli XL1-blue \\ E. coli One Shot Mach1-T1
}

\begin{abstract}
Invitrogen
Invitrogen

Stratagene

Invitrogen
\end{abstract}

\subsection{Mammalian cell lines}

HEK293T Human Embryonic Kidney immortalised cell line (ATCC)

(DuBridge et al., 1987)

HEK293FT Human Embryonic Kidney immortalised cell line (ATCC)

(Javanbakht et al., 2003)

PC12 Rat adrenal pheochromocytoma cell line (ATCC)

(Greene and Tischler, 1976)

Oli-neu Murine oligodendroglial precursor immortalised cell line

(Jung et al., 1995)

\subsection{Solutions and buffers}

Solutions were sterilised using an autoclave for $30 \mathrm{~min}$ at $120^{\circ} \mathrm{C}$ and $10^{5}$ Pascal. Heatsensitive solutions were sterile-filtered (pore size $0.2 \mu \mathrm{m}$ ). Consumables were autoclaved.

\subsubsection{Common buffers}

PBS (Phosphate buffered saline), 10x

$\mathrm{NaCl}$

$100 \mathrm{~g}$

$\mathrm{KCl}$

$2.5 \mathrm{~g}$

$\mathrm{Na}_{2} \mathrm{HPO}_{4}$ × $2 \mathrm{H}_{2} \mathrm{O}$

$7.2 \mathrm{~g}$

$\mathrm{KH}_{2} \mathrm{PO}_{4}$

$2.5 \mathrm{~g}$

Dissolve, adjust $\mathrm{pH}$ to 7.2 with $\mathrm{NaOH}$, add $\mathrm{ddH}_{2} \mathrm{O}$ to $1000 \mathrm{ml}$

TBS (Tris buffered saline), 10x 
Tris-Base (1 M; pH 8)

$50 \mathrm{ml}$ (f.c. $50 \mathrm{mM}$ )

$\mathrm{NaCl}(5 \mathrm{M})$

Dissolve, adjust $\mathrm{pH}$ to 7.4 with $\mathrm{HCl}$, add $\mathrm{ddH}_{2} \mathrm{O}$ to $1000 \mathrm{ml}$

\subsubsection{Buffers for genotyping}

MGB (Modified Gitschier buffer), 10x

Tris (1 M; $\mathrm{pH} 8.8)$

$6.7 \mathrm{ml}$ (f.c. $670 \mathrm{mM}$ )

$\left(\mathrm{NH}_{4}\right)_{2} \mathrm{SO}_{4}(1 \mathrm{M})$

$1.66 \mathrm{ml}$ (f.c. $166 \mathrm{mM})$

$\mathrm{MgCl}_{2}(1 \mathrm{M})$

$650 \mu \mathrm{l}$ (f.c. $65 \mathrm{mM}$ )

Add $\mathrm{ddH}_{2} \mathrm{O}$ to $10 \mathrm{ml}$

MGB, 1x

10x MGB

$2.2 \mathrm{ml}$

$10 \%$ Triton $\mathrm{X}-100$

$1.1 \mathrm{ml}$

Add $\mathrm{ddH}_{2} \mathrm{O}$ to $22 \mathrm{ml}$

To $180 \mu 1$ 1x MGB buffer $20 \mu 1$ proteinase $\mathrm{K}(10 \mathrm{mg} / \mathrm{ml})$ was added

\subsubsection{Buffers for molecular biology}

DNA sample buffer, $10 x$

$\begin{array}{ll}\text { Bromphenolblue } & 0.25 \% \\ \text { Xylencyanol } & 0.25 \% \\ \text { Ficoll (Type 400) } & 15 \%\end{array}$

In $\mathrm{ddH}_{2} \mathrm{O}$

TAE (Tris/Acetate/EDTA) buffer, 50x

Tris-Base $\mathrm{pH} 8$

EDTA (0.5 M; pH 8)

Add $\mathrm{ddH}_{2} \mathrm{O}$ to $1000 \mathrm{ml}$

TE (Tris-EDTA) buffer

Tris-Base (pH 7.4)

EDTA $(0.5 \mathrm{M})$

\section{Ethidiumbromide}

$\mathrm{EtBr} 1 \%$ in $\mathrm{ddH}_{2} \mathrm{O}$

Final concentration in the gel

dNTP mix, 50x

dATP, dCATP, dGTP, dTTP

F.c. in the PCR

\section{Primers}

Delivery concentration

F.c. in the PCR

\section{LB-medium (Luria and Bertani medium)}

Yeast extract

$10 \mathrm{mM}(2.5 \mathrm{mM}$ each $)$

$200 \mu \mathrm{M}(50 \mu \mathrm{M}$ each $)$

Bacto-Pepton pH 7.5
$10 \mathrm{mM}$

$1 \mathrm{mM}$

$10 \mathrm{mg} / \mathrm{ml}$

$1 \mu \mathrm{g} / \mathrm{ml}$

$50 \mathrm{pmol} / \mu \mathrm{l}$

$0.2 \mu \mathrm{M}$

$242 \mathrm{~g}(2 \mathrm{M})$

$100 \mathrm{ml}(1 \mathrm{mM})$

$0.5 \%(\mathrm{w} / \mathrm{v})$

$1 \%(\mathrm{w} / \mathrm{v})$ 


\section{SOC-medium}

Yeast extract

Bacto-Pepton pH 7.5

$0.5 \%(\mathrm{w} / \mathrm{v})$

$\mathrm{NaCl}$

$2 \%(\mathrm{w} / \mathrm{v})$

Glucose

$10 \mathrm{mM}$

$\mathrm{KCl}$

$20 \mathrm{mM}$

$\mathrm{MgSO}_{4}$

$2.5 \mathrm{mM}$

$10 \mathrm{mM}$

\section{Antibiotics}

f.c. in agar plate

Kanamycin

$50 \mu \mathrm{g} / \mathrm{ml}$

Ampicillin

$100 \mu \mathrm{g} / \mathrm{ml}$

\section{LB-agar plates}

Yeast extract

$0.5 \%(\mathrm{w} / \mathrm{v})$

Bacto-Pepton pH 7.5

$1 \%(\mathrm{w} / \mathrm{v})$

$\mathrm{NaCl}$

$1 \%(\mathrm{w} / \mathrm{v})$

Agar

Autoclave, cool down to $55^{\circ} \mathrm{C}$ in a water bath, add antibiotics

For blue-white selection add

X-Gal (5-bromo-4-chloro-indolyl- $\beta$-D-galactopyranoside)

IPTG (Isopropyl- $\beta$-D-thiogalactopyranoside)

\subsubsection{Luciferase assay buffers}

Passive lysis buffer

5x Passive lysis buffer from Promega diluted in $\mathrm{ddH}_{2} \mathrm{O}$

\section{Firefly luciferase assay buffer}

Tricine

$\left(\mathrm{MgCO}_{3}\right)_{4} * \mathrm{Mg}(\mathrm{OH})_{2} * 5 \mathrm{H}_{2} \mathrm{O}$

$\mathrm{MgSO}_{4}$

EDTA

DTT
$20 \mathrm{mM}$

$1.07 \mathrm{mM}$

$2.67 \mathrm{mM}$

$0.1 \mathrm{mM}$

$33.3 \mathrm{mM}$

Add $0.001 \mathrm{~V}$ of $37 \% \mathrm{HCl}$ to dissolve the magnesium carbonate When the solution becomes clear adjust the $\mathrm{pH}$ to 7.8 using $5 \mathrm{M} \mathrm{NaOH}$

Then add remaining components:

Coenzyme A

D-Luciferin, free acid

ATP
$270 \mu \mathrm{M}$

$470 \mu \mathrm{M}$

$530 \mu \mathrm{M}$

\section{Renilla luciferase assay buffer}

$\mathrm{NaCl}$

$1.1 \mathrm{M}$

$\mathrm{Na}_{2}$-EDTA

$2.2 \mathrm{mM}$

$\mathrm{KH}_{2} \mathrm{PO}_{4}(\mathrm{pH} 5.1)$

$0.22 \mathrm{M}$

BSA

$0.44 \mathrm{mg} / \mathrm{ml}$

$\mathrm{NaN}_{3}$

$1.3 \mathrm{mM}$

Adjust $\mathrm{pH}$ to 5.0 and add

Coelenterazin (dissolved in EtOH)

$1.43 \mathrm{mM}$ 
$\mathrm{KH}_{2} \mathrm{PO}_{4}$ (pH 5.1)

Prepare $1 \mathrm{M} \mathrm{KH}_{2} \mathrm{PO}_{4}$ solution, adjust $\mathrm{pH}$ to 5.1 using $2 \mathrm{M} \mathrm{KOH}$

\subsubsection{Solutions for cell culture}

2x Freezing medium for eukaryotic cell lines

$\begin{array}{ll}\text { DMEM } & 40 \% \\ \text { DMSO } & 20 \% \\ \text { FBS } & 40 \%\end{array}$

\section{PLL, 250x}

Poly L-lysin in $\mathrm{ddH}_{2} \mathrm{O}$

$5 \mathrm{mg} / \mathrm{ml}$

F.c. for PC12 and Oli-neu cells

$0.02 \mathrm{mg} / \mathrm{ml}$

F.c. for primary cultures

$0.1 \mathrm{mg} / \mathrm{ml}$

Penicillin-Streptomycin, 100x

Potassium penicillin

$10000 \mathrm{U} / \mathrm{ml}$

Streptomycin sulfate

$10000 \mu \mathrm{g} / \mathrm{ml}$

Trypsin-EDTA, 10x

Trypsin

$5 \mathrm{~g}$

EDTA

$6.84 \mathrm{mM}$

\subsubsection{Buffers for biochemistry}

NuPAGE MES SDS running buffer, 20x

MES (2-(N-Morpholino)propanesulfonic acid) $1 \mathrm{M}$

Tris-Base $1 \mathrm{M}$

SDS $2 \%$

EDTA $20 \mathrm{mM}$

Store at $4^{\circ} \mathrm{C}$

NuPAGE LDS sample buffer, $4 x$

Tris-Base

$564 \mathrm{mM}$

Tris-HCl

$424 \mathrm{mM}$

Lithium dodecyl sulfate

$8 \%(\mathrm{w} / \mathrm{v})$

EDTA (pH 8)

$2.04 \mathrm{mM}$

Glycerol

Serva Blue G250 (1\% solution)

$7.5 \%(\mathrm{v} / \mathrm{v})$

Phenol Red (1\% solution)

$2.5 \%(\mathrm{v} / \mathrm{v})$

Store at $-20^{\circ} \mathrm{C}$

\section{PonceauS staining buffer}

PonceauS

$0.2 \mathrm{~g}(0.2 \% \mathrm{w} / \mathrm{v})$

Trichloroacetic acid

$3 \mathrm{ml}(3 \% \mathrm{v} / \mathrm{v})$

Add $\mathrm{ddH}_{2} \mathrm{O}$ to $100 \mathrm{ml}$

NuPAGE transfer buffer (20x)

Bicine

Bis-Tris

EDTA

$20 \mathrm{mM}$ 
Chlorobutanol

Store at $4^{\circ} \mathrm{C}$

NuPAGE transfer buffer (1x)

NuPAGE Transfer Buffer (20x)

$100 \%$ methanol

Add $\mathrm{ddH}_{2} \mathrm{O}$ to $1000 \mathrm{ml}$

Stripping buffer

Glycine

Tween 20

Adjust to $\mathrm{pH} 2.5$ with $\mathrm{HCl}$

Washing buffer (TBS-T)

Tween-20

In TBS

\section{Blocking buffer}

Non fat milk powder

In TBS-T

\section{Primary antibody solution}

Appropriate antibody diluted in blocking buffer

Secondary antibody solution

Appropriate antibody diluted in blocking buffer

\section{Sucrose buffer}

Sucrose $(80 \%)$

Tris buffer (1 M, pH 7.4)

$\mathrm{NaHCO}_{3}(1 \mathrm{M})$

$0.2 \mathrm{M}$

$0.1 \%(\mathrm{v} / \mathrm{v})$

$50 \mathrm{ml}$

$150 \mathrm{ml}$

$\mathrm{MgCl}_{2}(2 \mathrm{M})$

Add $\mathrm{ddH}_{2} \mathrm{O}$ to $10 \mathrm{ml}$

Add freshly 1 tablet 'Complete Mini' protease inhibitors (Roche) to $10 \mathrm{ml}$ sucrose buffer

\subsubsection{Solutions and media for cell culture}

\section{Cell lines}

Media for culturing mammalian cells (DMEM 1g glucose/liter and DMEM $4.5 \mathrm{~g}$ glucose/liter) were purchased from Lonza. Prior to use and depending on the cell line media were supplemented with FCS and /or HS, both heat-inactivated at $56^{\circ} \mathrm{C}$ for $45 \mathrm{~min}$. All media contained 1\% penicillin/streptomycin solution and $200 \mathrm{mM}$ Glutamax-I (stable L-alanine-Lglutamine-dipeptide).

\section{Heterologous cell cultures}

Cell line
HEK293, HEK293FT
PC12

$\begin{array}{lll}\text { Medium } & \text { Percent FCS } & \text { Percent HS } \\ \text { DMEM } & 10 & - \\ \text { DMEM } & 10 & 10\end{array}$

\section{Primary cell cultures}

Sato medium for primary cultured oligodendrocytes 
For primary cultured oligodendrocytes a special medium (Sato) was prepared, which contained the following supplements in $500 \mathrm{ml}$ DMEM (4.5 g glucose/1):

Putrescine (10 mM stock soltution)

$5 \mathrm{ml}$

Glutamax-I (100x stock solution, Gibco)

$5 \mathrm{ml}$

Penicillin/Streptomycin (Lonza)

$5 \mathrm{ml}$

Insulin-Transferrin-Selenium-A (100x stock solution, Gibco) $5 \mathrm{ml}$

HS

$5 \mathrm{ml}$

Progesterone (2 $\mathrm{mM}$ stock solution)

$50 \mu 1$

Tri-Iodo-Thyrodine (500 $\mu \mathrm{M}$ stock solution)

$500 \mu 1$

L-Thyroxine (4 $\mathrm{mM}$ stock solution)

$65 \mu 1$

After shaking off, primary oligodendrocytes were plated in a modified Sato medium, which included the following ingredients in $100 \mathrm{ml}$ DMEM (4.5 g glucose/1):

$\begin{array}{ll}\text { B27 supplement (50x stock solution, Gibco) } & 2 \mathrm{ml} \\ \text { Glutamax-I (100x stock solution, Gibco) } & 1 \mathrm{ml} \\ \text { Penicillin/Streptomycin (Lonza) } & 1 \mathrm{ml} \\ \text { Pyruvate solution }(1.1 \%) & 1 \mathrm{ml} \\ \text { HS } & 1 \mathrm{ml} \\ \text { Tri-Iodo-Thyrodine (500 } \mu \text { M stock solution) } & 10 \mu l \\ \text { L-Thyroxine (4 mM stock solution) } & 13 \mu l\end{array}$

\section{Neuron plating medium}

Neurobasal medium (Gibco)

B27 supplement

Penicillin/Streptomycin

$500 \mathrm{ml}$

$2 \%$

FCS

$1 \%$

Glucose

$5 \%$

$0.3 \%$

$\mathrm{NaCl}$

$37.5 \mathrm{mM}$

\section{BME/HS (Whole brain cultures)}

$\begin{array}{ll}\text { BME } & 500 \mathrm{ml} \\ \text { HS } & 10 \%(50 \mathrm{ml}) \\ \text { Penicillin/Streptomycin } & 1 \%(5 \mathrm{ml}) \\ \text { Glutamax-I } & 1 \%(5 \mathrm{ml})\end{array}$

Trypsin/EDTA (10x, $100 \mathrm{ml}$ stock solution)

Trypsin

$5 \mathrm{~g}$

EDTA

$6.84 \mathrm{mM}$

Trypsin-EDTA for primary cultures

10x stock diluted to 1x with BME medium (Gibco)

Transfection medium

OptiMEM-Medium (Gibco)

\section{DNAseI solution}

Dissolve $100 \mathrm{mg}$ DNAseI (Roche) in $200 \mathrm{ml} \mathrm{HBSS}+$ to obtain a $0.5 \mathrm{mg} / \mathrm{ml}$ stock solution $(0.05 \%)$

Store aliquots at $-20^{\circ} \mathrm{C}$ 
HBSS+ $\left(4^{\circ} \mathrm{C}\right)$

HBSS (BioWhittaker Cambrex) $500 \mathrm{ml}$

$\mathrm{MgSO}_{4}(10 \%(\mathrm{w} / \mathrm{v})$, sterile filtered) $\quad 7.5 \mathrm{ml}$

\subsubsection{Buffers for immunohistochemistry and immunocytochemistry}

\section{Citrate buffer}

Stock solution A

Citric acid

Stock solution B

Sodium citrate

Citrate buffer working solution

Stock solution A

$9 \mathrm{ml}$

Stock solution B

$41 \mathrm{ml}$

Add $\mathrm{ddH}_{2} \mathrm{O}$ to $500 \mathrm{ml}$

\section{Tris buffer}

Stock solution (0.5 M, pH 7.6)

TRIS tris(hydroxymethyl)aminomethane

$60.75 \mathrm{~g}$

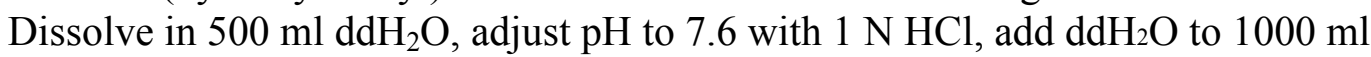

Working solution (0.05 M, pH 7.6)

Stock solution

$\mathrm{NaCl}$

Add $\mathrm{ddH}_{2} \mathrm{O}$ to $1000 \mathrm{ml}$

Tris buffer $+2 \%$ non fat milk powder $(1000 \mathrm{ml})$

Tris buffer working solution

Non fat milk powder

Filtrate

\section{Phosphate buffer according to Sorensen}

Stock solution A

Monosodium phosphate

Stock solution B

Disodium phosphate

Phosphate buffer working solution

Stock solution A

Stock solution B

$\mathrm{ddH}_{2} \mathrm{O}$

\section{$0.2 \mathrm{M}$}

$20 \mathrm{ml}$

$80 \mathrm{ml}$

$100 \mathrm{ml}$

$9 \mathrm{~g}$

$1000 \mathrm{ml}$

$20 \mathrm{~g}$

$100 \mathrm{ml}$

Fixation solution for immunohistochemistry $(1000 \mathrm{ml})$

Phosphate buffer stock solution A

$100 \mathrm{ml}$

Phosphate buffer stock solution B $\quad 400 \mathrm{ml}$

$37 \%$ Formol, filtered $\quad 100 \mathrm{ml}$

$\mathrm{ddH}_{2} \mathrm{O} \quad 400 \mathrm{ml}$ 
Bovine Serum Albumin in PBS (PBS/BSA)

Phosphate buffer stock solution A

$20 \mathrm{ml}$

Phosphate buffer stock solution B

$80 \mathrm{ml}$

$\mathrm{NaCl}$

BSA

$1.8 \mathrm{~g}$

$1 \mathrm{~g}$

Add $\mathrm{ddH}_{2} \mathrm{O}$ to $100 \mathrm{ml}$

\section{Blocking buffer}

FCS

$2 \%(\mathrm{v} / \mathrm{v})$

BSA

$2 \%(\mathrm{w} / \mathrm{v})$

Fish gelantine (Simga)

$2 \%(\mathrm{w} / \mathrm{v})$

In PBS

\section{Permeabilisation buffer}

Triton-X-100

In PBS

$0.1 \%(\mathrm{v} / \mathrm{v})$

\section{Antibody dilution}

Blocking buffer

In PBS

$10 \%(\mathrm{v} / \mathrm{v})$

Antibody in appropriate dilution

DAPI solution

4',6-diamidino-2-phenylindole

in $\mathrm{H}_{2} \mathrm{O}$

$1 \mathrm{mg} / \mathrm{ml}$

dilute 1:2000 


\section{Methods}

\subsection{Molecular biology methods}

Molecular biology standard methods were performed according to Sambrook and Russel (Sambrook and Russell, 2001) and to the manufacturer's instructions if not indicated otherwise.

\subsubsection{DNA transfer in E.coli}

\subsubsection{Transformation of chemically competent bacteria}

Aliquots of transformation-competent bacteria (E.coli, strain XL1 blue, DH5a, Mach1) were thawed on ice. 5-10 $\mu \mathrm{l}$ of a ligation or recombination reaction was added to $100 \mu \mathrm{l}$ cells, followed by an incubation step on ice for $30 \mathrm{~min}$. The bacteria were heat-shocked at $42^{\circ} \mathrm{C}$ for $40 \mathrm{~s}$, and then cooled on ice for $2 \mathrm{~min} .800 \mu \mathrm{l}$ cold LB medium without antibiotics was added, and the mix was incubated at $37^{\circ} \mathrm{C}$ for 45 min with moderate shaking. During this incubation the cells start expressing the appropriate antibiotic resistance. Then, 100-800 $\mu 1$ were plated on a LB-agar plate containing the appropriate antibiotic. Sterile glass beads were used to equally distribute the bacteria. The plates were incubated overnight at $30-37^{\circ} \mathrm{C}$.

\subsubsection{Electroporation of bacteria}

The commercially available electro-competent E.coli strain DH10B was diluted 1:4 with sterile $10 \%$ glycerol, and $20 \mu \mathrm{l}$ aliquots were made. For electroporation, the bacteria were thawed on ice, $2-2.5 \mu \mathrm{l}$ of a recombination or ligation reaction was added, and the mix was transferred into a $1 \mathrm{~mm}$ electroporation cuvette (BioRad). The electroporation was performed using the 'GenePulserII' (BioRad) with the following settings: $1.75 \mathrm{kV}, 25 \mu \mathrm{F}$ capacitance and $200 \Omega$ resistance. The cells were resuspended in $800 \mu 1$ cooled LB, or alternatively in SOC medium, without antibiotics and incubated at $37^{\circ} \mathrm{C}$ for $45 \mathrm{~min}$ with moderate shaking. The bacteria were plated as described above in the section 'Transformation of chemically competent bacteria'.

\subsubsection{Isolation and analysis of DNA and RNA}

Plasmid DNA preparations are based on a modified protocol of Birnboim and Doly (Birnboim and Doly, 1979), depending on the alkaline cell lysis followed by SDS precipitation of proteins and genomic DNA. The plasmid DNA was bound to a silica membrane under appropriate high-salt conditions, washed and finally eluted. Plasmid DNA was isolated from fresh overnight bacterial LB cultures grown to an OD of 2 to 3 using the Plasmid DNA Purification Kits (Mini and Midi scale) provided by Macherey-Nagel. Detailed description of the procedures can be found in the manuals provided by the manufacturers.

\subsubsection{Plasmid DNA mini preparations}

In brief, the pelleted bacteria (2-4 $\mathrm{ml}$ of the overnight culture) were resuspended in $250 \mu 1$ buffer A1, and cell lysis was performed by adding $250 \mu 1$ buffer A2. After 5 min incubation the reaction was stopped by adding $300 \mu 1$ neutralisation buffer A3. The mix was centrifuged 
for 5 min to pellet genomic DNA as well as proteins, and the clear supernatant was applied onto the column. The resin was washed with $450 \mu \mathrm{l}$ buffer $\mathrm{AQ}$, dried by centrifugation, and finally, the plasmid DNA was eluted in $100 \mu \mathrm{l}$ water or TE buffer.

\subsubsection{Plasmid DNA midi preparations}

In brief, pelleted bacteria (100 $\mathrm{ml}$ of the overnight culture) were resuspended in $4 \mathrm{ml}$ buffer S1. $4 \mathrm{ml}$ buffer A2 was added and after 2-3 min incubation at RT cell lysis was stopped by adding $4 \mathrm{ml}$ precooled buffer S3. The suspension was incubated on ice for $5 \mathrm{~min}$. In the meantime, the column was equilibrated with $2.5 \mathrm{ml}$ buffer $\mathrm{N} 2$, and the filtered clear lysate was loaded. The column was washed with $10 \mathrm{ml}$ buffer N3, and the DNA was eluted in $5 \mathrm{ml}$ buffer N5. Finally, the DNA was precipitated using isopropanol, and it was resuspensed in $100 \mu 1 \mathrm{TE}$ buffer.

\subsubsection{Genomic DNA preparation from mouse tissue}

Genomic DNA (up to $50 \mu \mathrm{g}$ ) was prepared from mouse tail biopsy material (appr. $0.5 \mathrm{~cm}$ ) or other tissues. The samples were incubated in $180 \mu 1$ 1x MGB buffer plus $20 \mu 1$ proteinase K $(10 \mathrm{mg} / \mathrm{ml})$ for 4 hours at $55^{\circ} \mathrm{C}$ and $1000 \mathrm{rpm}$. Afterwards, the mix was vortexed and the proteinase $\mathrm{K}$ was heat-inactivated for $10 \mathrm{~min}$ at $95^{\circ} \mathrm{C}$. Cell debris was removed by centrifugation at $2000 \mathrm{~g}$ for $10 \mathrm{~min} .2 \mu \mathrm{l}$ were used for the genotyping PCR.

\subsubsection{RNA micro preparation from micro-dissected material}

Small amounts of RNA (up to $45 \mathrm{ng}$ ) were isolated using the RNeasy Micro Kit from Qiagen. The kit is based on the selective binding of RNAs $>200$ bases to a silica membrane under high-salt conditions, which excludes 5S, 5.8S and tRNAs.

Micro-dissected tissue was lysed in $350 \mu \mathrm{l}$ buffer RLT and stored at $-80^{\circ} \mathrm{C}$ for further analysis. For RNA isolation, the samples were quickly thawn by warming them up to $37^{\circ} \mathrm{C}$ for $10 \mathrm{~min}$, thoroughly vortexed, and spun down to remove all material from the cap. An equal volume $(350 \mu 1)$ of $70 \%$ ethanol was added, and the mix was immediately applied to the column. After three washing steps, which included $700 \mu 1$ buffer RW1, $500 \mu 1$ buffer RPE, and $500 \mu 180 \%$ ethanol, the column was dried by centrifugation, and the RNA was eluted in $100 \mu 1$ RNase free water.

\subsubsection{RNA mini preparation from primary cultured oligodendrocytes}

To harvest cells from a 24 well plate the medium was removed and $350 \mu$ RLT buffer per well was applied. The samples were transferred into eppendorf tubes and vortexed thoroughly to ensure complete lysis. Samples were stored at $-80^{\circ} \mathrm{C}$ for further analysis. For RNA preparation, the RNeasy Mini Kit from Qiagen was used. The lysates were quickly thawn at $37^{\circ} \mathrm{C}$ for $10 \mathrm{~min}$, vortexed and spun down before they were loaded onto a QIAshredder column (Qiagen) for optimal homogenisation. The column was centrifuged at $13000 \mathrm{rpm}$, and an equal volume $(350 \mu 1)$ of $70 \%$ ethanol was added to the eluate. The RNeasy column was loaded with the mix and washed with $350 \mu \mathrm{l}$ buffer RW1. For material from cell culture, an on-column DNaseI digest ( $10 \mu \mathrm{l}$ DNaseI plus $70 \mu \mathrm{l}$ buffer RDD) for 15 min was performed to avoid DNA contamination. Subsequent wash steps with $350 \mu$ l buffer RW1 and 2x $500 \mu 1$ buffer RPE were performed before the column was dried. The RNA was eluted in $100 \mu 1$ RNase-free water. 


\subsubsection{Precipitation of RNA}

Following the RNA micro and mini preparation, the RNA was precipitated by adding a carrier (glycogen), a monovalent salt (ammonium acetate) and 100\% ethanol. The $100 \mu$ l eluate was mixed thoroughly with $1 \mu \mathrm{l}$ glycogen solution $(20 \mathrm{mg} / \mathrm{ml}), 50 \mu \mathrm{l}$ of $7.5 \mathrm{M} \mathrm{NH}_{4} \mathrm{Ac}$ and $2-2.5 \mathrm{x}$ volume $(375 \mu \mathrm{l})$ of $100 \%$ ethanol. The samples were centrifuged at $4{ }^{\circ} \mathrm{C}$ for $30 \mathrm{~min}$ at 13000 rpm and washed with 70\% ethanol. The air-dried pellet was resuspended in $4 \mu 1$ RNase-free water for cDNA synthesis.

\subsubsection{Photometric concentration determination of nuclear acids}

Concentration and purity of a nucleic acids solution can be analysed by spectrophotometry. According to the Lambert-Beer law, the concentration of a solution is directly proportional to its extinction or absorption, that is

$$
\mathrm{A}=\varepsilon * \mathrm{c} * 1
$$

with $\varepsilon$ representing the molar extinction coefficient (unit $\mathrm{M}^{-1} \mathrm{~cm}^{-1}$ ), $c$ being the concentration (unit $\mathrm{M}$ ) and $l$ being the optic path length (cuvette thickness) that the light passes through the sample (unit $\mathrm{cm}$ ). The extinction coefficients for nuclear acids at $\lambda=260 \mathrm{~nm}$ are

$$
\begin{aligned}
& \text { guanine: } \varepsilon=12010 \mathrm{M}^{-1} \mathrm{~cm}^{-1} \\
& \text { cytosine: } \varepsilon=7050 \mathrm{M}^{-1} \mathrm{~cm}^{-1} \\
& \text { adenine: } \varepsilon=15200 \mathrm{M}^{-1} \mathrm{~cm}^{-1} \\
& \text { thymine: } \varepsilon=8400 \mathrm{M}^{-1} \mathrm{~cm}^{-1} \text { (uracile: } \varepsilon=8111 \mathrm{M}^{-1} \mathrm{~cm}^{-1} \text { ) }
\end{aligned}
$$

Using a spectrophotometer, the absorption at $260 \mathrm{~nm}$ (maximum of absorption of nucleic acids) and $280 \mathrm{~nm}$ (maximum of absorption of aromatic amino acids in proteins) of the nucleic acid solution was determined. For a reliable measurement, it is of importance that the value for the absorption at $260 \mathrm{~nm}$ is set between 0.1 and 1 to fit the linear range of the photometer. Therefore, a proper dilution of the sample was necessary. To determine the concentration the following relation was applied:

$$
\begin{aligned}
& 1 \mathrm{OD}_{260} \text { (optical density at } 260 \mathrm{~nm} \text { ) }=50 \mathrm{mg} / \mathrm{ml} \text { for double stranded (ds) DNA } \\
& 1 \mathrm{OD}_{260} \quad=40 \mathrm{mg} / \mathrm{ml} \text { for single stranded (ss) RNA } \\
& 1 \mathrm{OD}_{260} \quad=33 \mathrm{mg} / \mathrm{ml} \text { for ss oligonucleotides }
\end{aligned}
$$

The equation to determine the concentration of dsDNA was

$$
\mathrm{c}(\mu \mathrm{g} / \mu \mathrm{l})=\mathrm{OD}_{260} * 50 * \text { dilution factor } / 1000
$$

The purity of a given sample was assessed by the relative absorption values of $260 \mathrm{~nm}$ over $280 \mathrm{~nm}$. Clean preparations of DNA and RNA would return values between 1.8 and 2, whereas significantly lower values indicated a protein contamination.

\subsubsection{Agarose gel electrophoresis}

Agarose gels of $0.8 \%-2.5 \%$ were used to separate DNA fragments between $0.3 \mathrm{~kb}$ and $15 \mathrm{~kb}$ of size. The proper amount of agarose was added to $1 \mathrm{x}$ TAE buffer, and the suspension was heated in the microwave for appr. $10 \mathrm{~min}$ until the agarose was completely dissolved. The solution was cooled to $60^{\circ} \mathrm{C}$ to minimise evaporation, and ethidium bromide was added to a 
final concentration of $1 \mu \mathrm{g} / \mu \mathrm{l}$. The fluid agarose was poured into a gel-casting form, and combs were inserted to define the pockets for sample loading. The agarose needed 20-30 min to solidify. Next, the gel was placed in a chamber containing 1x TAE buffer. The DNA samples were prepared by adding the proper volume of 10x loading dye and loaded into the pockets of the gel. The voltage applied was 30-160 V (5-10 V/cm), depending on the size of the gel chamber and the agarose concentration used. DNA molecules migrate to the positive electrode as they are negatively charged, thus resulting in their size-dependent separation. The DNA could be visualised by the fluorescence of the incorporated ethidium bromide when exposed to an UV light source emitting $260 \mathrm{~nm}$. The $100 \mathrm{bp}$ marker or $1 \mathrm{~kb}$ marker (Fermentas, St. Leon-Rot) were used as molecular size standards. Gel chamber, casting form and combs were produced by the workshop of the Max-Planck-Institute.

\subsubsection{Isolation of DNA from agarose gels}

To isolate DNA from agarose gels a gel extraction kit provided by Macherey-Nagel was used. The area of the gel containing the desired DNA was excised under UV light (356 nm, Intas UV systems, Heidelberg) with a scalpel, weighted and transferred to an Eppendorf reaction tube. The DNA was purified according to the manufacturer's instructions.

In brief, for each $100 \mathrm{mg}$ of agarose gel, $200 \mu \mathrm{l}$ of buffer NTI was added. The mix was incubated for $5-10 \mathrm{~min}$ at $50^{\circ} \mathrm{C}$ until the agarose was dissolved completely. The sample was loaded onto a silica membrane, washed with $700 \mu$ l buffer NT3, dried, and finally eluted in 30-50 $\mu 1$ buffer NE.

\subsubsection{DNA digest with restriction endonucleases}

DNA restriction digests were performed using type II restriction endonucleases in reaction volumes of 30-200 $\mu 1$. Type II restriction endonucleases induce cleavage mostly within their recognition sequences of 4-8 nucleotides, and yield in 5'- or 3'-DNA overhangs or blunt ends depending on the enzyme. Most enzymes were supplied with one of four standard 10x reaction buffers (NEB, New England Biolabs). 0.5 to $1 \mu \mathrm{g}$ or up to $5 \mu \mathrm{g}$ of DNA were used for analytical or preparative digests, respectively. The restriction enzyme unit ' $U$ ' defines the amount of enzyme that is required to cut $1 \mu \mathrm{g}$ DNA in 1 hour at $37^{\circ} \mathrm{C}$. Usually, 2- to 3-fold the amount of enzyme was used in digests.

\subsubsection{DNA sequencing}

DNA sequencing was performed at the Institute's DNA Core Facility (Department of Neurobiology, MPI of Experimental Medicine, Göttingen) lead by Dr. Fritz Benseler. DNA sequencing is based on a modified dideoxy chain reaction termination method according to Sanger (Sanger et al., 1977). The approach is based on a linear DNA amplification in the presence of a sequencing primer, a DNA polymerase, normal deoxynucleoside triphosphates (dNTPs), and modified nucleotides (dideoxyNTPs) that terminate DNA strand elongation. DideoxyNTPs stochastically incorporate into the newly synthesized DNA by replacing the normal dNTPs thereby terminating the synthesis. The products of different length are separated by capillary electrophoresis. As each of the four dideoxyNTPs is coupled to a different fluorescent dye the DNA fragments can be characterised by size, and the terminal nucleotide is identified. Through a repetitive procedure, the DNA sequence is determined. 


\subsubsection{Sequence analysis of DNA}

Sequencing data was analysed using the DNAstar software, version 8, provided by Lasergene. The obtained sequences were also aligned to databases of the "National Center for Biotechnology Information' (NCBI, http://www.ncbi.nlm.nih.gov) and ENSEMBL (http://www.ensembl.org).

\subsubsection{Modification of DNA}

\subsubsection{Ligation of DNA}

The enzyme DNA ligase derived from the bacteriophage T4 catalises the formation of covalent phosphodiester bonds between free 3'-hydroxy and 5'-phosphate overhangs of double stranded DNA. Cofactors needed for this reaction are $\mathrm{Mg}^{2+}$ ions and ATP. The cloning of DNA fragments, that is the ligation of insert and vector, was performed overnight at $4{ }^{\circ} \mathrm{C}$. The 10-20 $\mu 1$ of a ligation reaction contained 50-100 ng vector DNA, 150-300 ng insert DNA (molar ratio 1:3), 1.5 U T4 DNA ligase, and the ATP-containing 10x buffer provided by the manufacturer. The reaction was stopped by incubation at $65^{\circ} \mathrm{C}$ for $10 \mathrm{~min}$.

Standard ligation reaction:

$\mathrm{x} \mu \mathrm{l}$ vector (50-100 ng DNA)

$\mathrm{x} \mu \mathrm{l}$ insert (150-300 ng DNA)

$1 \mu 110 \mathrm{x}$ buffer

$0.5 \mu 1 \mathrm{~T} 4$ ligase $(1.5 \mathrm{U})$

Add $10 \mu \mathrm{ldd} \mathrm{H}_{2} \mathrm{O}$

\subsubsection{Dephosphorylation of 5'-DNA fragment overhangs}

The terminal 5'-phosphate groups of dsDNA can be removed by phosphatase treatment using the enzyme calf intestinal alkaline phosphatase (CIP). This helps to reduce the likelihood of unwanted vector religations. As CIP is compatible with NEB-, Roche- and Promega-buffers 1-2 $\mu 1$ of the enzyme was added to a completed DNA digest and incubated at $37^{\circ} \mathrm{C}$ for maximal $20 \mathrm{~min}$. To stop the reaction the digested DNA fragment was purified by agarose electrophoresis.

\subsubsection{Cloning of PCR products}

PCR products were purified by agarose electrophoresis and using a gel extraction kit (Macherey-Nagel). The PCR products were, if they contained terminal restrictions sites, first cut with the appropriate restriction enzyme(s), purified, and then used for ligation into the designated vector. PCR products amplified with Taq polymerase were also directly subcloned into the pGemT vector (Promega) through so-called TA cloning. The technique is based on the property of the Taq polymerase to attach an additional nucleotide (preferentially adenosine) to the 3 '-end of the completed PCR product. The pGemT vector can be purchased in a linearised form with complementary 5'-thymine overhangs which bind to the PCR fragment. If a proofreading polymerase with 3 '-exonuclease activity (e.g. $P f u$ polymerase, Stratagene) was used in the PCR, the adenosine overhangs had to be added in a second reaction using the Taq polymerase. To do this, $25 \mu 1$ of the purified PCR product were incubated with $5 \mu 1$ 10x buffer, dATP (f.c. $0.2 \mathrm{mM}$ ), and $5 \mathrm{U}$ Red Taq polymerase in a final reaction volume of $50 \mu \mathrm{l}$ incubated for $10 \mathrm{~min}$ at $37^{\circ} \mathrm{C}$. The reaction was purified with the 
help of the PCR clean-up kit by Macherey-Nagel. Therefore, $200 \mu 1$ buffer NTI was added per $100 \mu 1$ PCR reaction volume, the mix was applied onto the column, washed with buffer NT3 dried, and finally eluted in 30-50 $\mu$ l buffer NE. 5-7 $\mu 1$ were used for ligation.

\subsubsection{Amplification of DNA by polymerase chain reaction}

The polymerase chain reaction (PCR) was developed by Kay Mullis (Mullis et al., 1986), and is now a commonly used method for selective in vitro amplification of defined DNA fragments. The DNA synthesis begins with the annealing of two DNA oligonucleotides (primers), which flank the DNA stretch that is supposed to be amplified. One primer anneals as a sense primer to the plus strand of the DNA, the other primer binds as an anti-sense primer to the minus strand. The polymerase recognises the dsDNA (primer and template) and exponentially amplifies the DNA region flanked by the primers. The synthesis is catalysed by thermostable Taq polymerases (derived from the thermophilic bacterium Thermus aquaticus) or proofreading polymerases (e.g. $P f u$ polymerase, Stratagene, derived from the thermophilic archaea bacterium Pyrococcus furiosus). The latter one helps to reduce the mutation rate by its 5 '-3'-exonuclease activity by one power to about one mutation in $10^{6}$ nucleotides.

Standard PCR reaction $(20 \mu \mathrm{l})$ :

$\mathrm{x} \mu \mathrm{l}$ template $(10-100 \mathrm{ng})$

$1 \mu 1$ sense (forward) primer $(10 \mathrm{pmol} / \mu \mathrm{l})$

$1 \mu 1$ anti-sense (reverse) primer $(10 \mathrm{pmol} / \mu \mathrm{l})$

$2 \mu 110 x$ reaction buffer (including $\mathrm{MgCl}_{2}$ )

$5 \mu 1 \mathrm{dNTP}$ mix (dATP, dCTP, dGTP, dTTP; f.c. $0.2 \mathrm{mM}$ each)

$0.8 \mu \mathrm{l} \mathrm{Taq}$ polymerase $(5 \mathrm{U} / \mu \mathrm{l})$

Add $\mathrm{ddH}_{2} \mathrm{O}$ to $20 \mu \mathrm{l}$

A PCR reaction consists of three main steps: 1. Denaturation, 2. primer annealing, and 3. elongation. The PCR steps are run at different temperatures, and they are repeated multiple times in cycles. At the beginning of the PCR, an initial denaturation at $95^{\circ} \mathrm{C}$ was used to remove any DNA secondary structures followed by a primer annealing step between $50-60^{\circ} \mathrm{C}$ where the primers are supposed to bind to their complementary DNA sequence. The annealing temperature was $2-4^{\circ} \mathrm{C}$ lower than the computed melting temperature of the primers. At $72^{\circ} \mathrm{C}$ the elongation was performed, which is the optimum temperature for the Taq polymerase. The extension time was calculated depending on the template length and the polymerase used. Non proofreading polymerases require up to $30 \mathrm{sec}$ per $1 \mathrm{~kb}$ template, whereas proofreading enzymes need about 1 min per $1 \mathrm{~kb}$. The PCR ended in a final elongation step at $72^{\circ} \mathrm{C}$ to ensure complete synthesis. Depending on the DNA template and the purpose (cDNA, plasmid DNA, genomic DNA) 15-36 cycles were run. The volume of the PCR reaction was $20 \mu \mathrm{l}$ (genotyping PCR) or $50 \mu \mathrm{l}$ (preparative PCR).

Standard PCR protocol

3 min $\rightarrow 95^{\circ} \mathrm{C}$, initial denaturation

$30 \mathrm{~s} \rightarrow 56^{\circ} \mathrm{C}$, annealing

$60 \mathrm{~s} \rightarrow 72^{\circ} \mathrm{C}$, elongation

$30 \mathrm{~s} \rightarrow 95^{\circ} \mathrm{C}$, denaturation

cycle

$60 \mathrm{~s} \rightarrow 55^{\circ} \mathrm{C}$, final annealing

$10 \mathrm{~min} \rightarrow 72^{\circ} \mathrm{C}$, final elongation 


\subsubsection{Designing primer sequences}

Primers were designed with melting temperatures $\left(\mathrm{T}_{\mathrm{m}}\right)$ of $60^{\circ} \mathrm{C}$. Primer melting temperature can be roughly calculated with the help of the following formula:

$\mathrm{T}_{\mathrm{m}}=(\mathrm{A}+\mathrm{T}) 2+(\mathrm{G}+\mathrm{C}) 4$, with $\mathrm{A}, \mathrm{T}, \mathrm{G}$ and $\mathrm{C}$ indicating the numbers of the corresponding nucleotides in the primer sequence. For a more precise $\mathrm{T}_{\mathrm{m}}$ determination, an online algorithm developed by Warren $\mathrm{A}$. Kibbe (Kibbe, 2007) was used (http://www.basic.northwestern.edu/biotools/oligocalc.html).

\subsubsection{Site-directed mutagenesis}

This method of PCR-based in vitro mutagenesis presents a fast approach to exchange and manipulate one or more nucleotides, also simultaneously, in a DNA molecule. Following aspects for the design of the mutagenesis oligonucleotides have to be considered:

- The length of the oligonucleotide is supposed to be in the range between 24 and 45 bp

- The $\mathrm{T}_{\mathrm{m}}$ of the oligonucleotide should be higher than $78^{\circ} \mathrm{C}$ and is calculated by using the following formula:

$\mathrm{T}_{\mathrm{m}}=81.5+0.41(\% \mathrm{GC})-675 / \mathrm{N}-\%$ mismatch

(with $\mathrm{N}$ being the length of the oligonucleotide in bp and \% GC reflecting the GC content in percent)

- The mutation, deletion or insertion should preferentially lie in the middle the oligonucleotide and flanked by each side with 10-15 bp which exhibit $100 \%$ complementarity to the target sequence

- The GC content of the oligonucleotide should not be below $40 \%$

- The oligonucleotide should start and end with at minimum one $\mathrm{G}$ or $\mathrm{C}$

The mutagenesis PCRs are exclusively performed using proofreading polymerases. The PCR product was digested with $D p n I$, a restriction endonuclease, which specifically recognises the methylated sequence 5 ' $\mathrm{G}_{\mathrm{m} 6} \mathrm{ATC} 3$ ', and therefore cuts only the parental template DNA generated in E.coli. The mutagenised sample was gel purified and transformed into bacteria for amplification.

\subsubsection{7 cDNA synthesis}

Ss cDNA was generated from RNA isolated from mouse tissue or cell culture or lentiviral RNA using either SuperscriptIII reverse transcriptase (Invitrogen) or the Transcriptor High Fidelity cDNA Synthesis Kit (Roche). In the latter, the catalising enzyme was a new recombinant reverse transcriptase with proofreading activity. If there was only very little amount of starting material available the total RNA was reversed transcribed using the Roche kit. When handling with larger amounts, usually 0.5-1 $\mu \mathrm{g}$, SuperscriptIII used.

Protocol using SuperscriptIII

Total RNA, or a fraction thereof, was precipitated and resuspended in $4 \mu \mathrm{RNase}$ free $\mathrm{H}_{2} \mathrm{O}$ as described above. $1 \mu \mathrm{l}$ of anchored oligo-dT primer $(0.6 \mathrm{pmol} / \mu \mathrm{l})$ and $1 \mu \mathrm{l}$ of the random nonamer N9 primer $(120 \mathrm{pmol} / \mu \mathrm{l})$ were added. The oligo-dT primers are specific to the small number of polyadenylated mRNAs (1-2\%) in the whole RNA pool, whereas the random primer is added to get a more efficient transcription in 5'-mRNA regions. The ratio applied between oligo-dT and random primer was 1:200. The RNA/primer mixture was denaturated for $2 \mathrm{~min}$ at $70^{\circ} \mathrm{C}$ and then cooled on ice. Then, the other components were added: 
$2 \mu 1 \quad 5 \mathrm{x} 1 \mathrm{st}$ strand buffer

$1 \mu \mathrm{l} \quad \mathrm{DTT}(0.1 \mathrm{M})$

$0.5 \mu \mathrm{l}$ dNTPs $(10 \mathrm{mM}$ each)

$1 \mu \mathrm{l} \quad$ Superscript III $(200 \mathrm{U} / \mu \mathrm{l})$

$10 \mu \mathrm{l}$ final volume

The reaction was incubated for $10 \mathrm{~min}$ at $25^{\circ} \mathrm{C}$ to allow for the annealing of primers. Further, incubation steps for $45 \mathrm{~min}$ at $50^{\circ} \mathrm{C}$ followed by another $45 \mathrm{~min}$ at $55^{\circ} \mathrm{C}$ were carried out for the RNA-dependent cDNA synthesis. The obtained cDNA was diluted in an appropriate volume of water for quantitative real-time-PCR analysis.

Protocol using the transcriptor high fidelity cDNA synthesis kit (Roche)

As described above, the total RNA resuspended in $4 \mu 1 \mathrm{H}_{2} \mathrm{O}$ was used for synthesis. $1 \mu \mathrm{l}$ of anchored oligo-dT-N18 primer $(50 \mathrm{pmol} / \mu \mathrm{l})$ and $2 \mu \mathrm{l}$ of random hexamer primer $(600$ $\mathrm{pmol} / \mu \mathrm{l}$ ) plus $4.5 \mu \mathrm{l} \mathrm{H}_{2} \mathrm{O}$ were added and incubated for $10 \mathrm{~min}$ at $65^{\circ} \mathrm{C}$. The reaction was cooled down on ice and mixed with the following substances:

$4 \mu 1 \quad 5 \mathrm{x}$ reaction buffer

$1 \mu 1 \quad$ DTT $(0.1 \mathrm{M})$

$2 \mu \mathrm{l}$ dNTPs (10mM each)

$0.5 \mu \mathrm{l}$ protector RNase inhibitor $(40 \mathrm{U} / \mu \mathrm{l})$

$1.1 \mu \mathrm{l}$ reverse transcriptase (appr. $200 \mathrm{U} / \mu \mathrm{l}$ )

$20 \mu \mathrm{l}$ final volume

The mixture was incubated for $30 \mathrm{~min}$ at $45^{\circ} \mathrm{C}$, and $30 \mathrm{~min}$ at $55^{\circ} \mathrm{C}$ before the enzyme was denaturated at $85^{\circ} \mathrm{C}$ for $5 \mathrm{~min}$. The obtained cDNA was diluted in an appropriate volume of water for quantitative real-time-PCR analysis.

\subsubsection{Quantitative real-time-PCR}

Quantitative real-time-PCR (qRT-PCR) is an amplification method for nucleic acids which is based on the principle of standard PCR but allows additional quantification.

\section{SYBR Green assays}

The fluorescent dye SYBR Green (Applied Biosystems) intercalates into dsDNA and facilitates a quantitative analysis during the cycles (real-time). The fluorescence increases proportional to the amount of PCR product generated.

\section{TaqMan assays}

In this thesis, TaqMan assays (Applied Biosystems) were also performed. They require an additional dual-labelled probe (with a fluorophore and a quencher) complementary to the target sequence. The probe is cleaved by the 5'-3'exonuclease activity of the Taq polymerase, thereby stopping the quenching effect and allowing fluorescence of the fluorophore to form. TaqMan probes significantly increase the specificity of the detection.

The qRT-PCR was performed in specially designated 96-well plates (Applied Biosystems) using the Fast Real-Time-PCR System (Applied Biosystems). Primers for qRT-PCR were designed using the Roche Universal Probe Library (https://www.roche-applied-science.com).

A standard SYBR Green reaction included: 
$4 \mu 1$ ssRNA $(\mathrm{x} \mu \mathrm{g})$

$5 \mu 12 x$ SYBR Green master mix (including SYBR Green and Taq polymerase)

$0.1 \mu \mathrm{l}$ fwd primer $(10 \mathrm{pmol} / \mu \mathrm{l})$

$0.1 \mu 1 \mathrm{rev}$ primer $(10 \mathrm{pmol} / \mu \mathrm{l})$

$0.8 \mu 1 \mathrm{H}_{2} \mathrm{O}$

A standard TaqMan reaction contained:

$4 \mu \mathrm{l}$ ssRNA $(\mathrm{x} \mu \mathrm{g})$

$5 \mu 12 \mathrm{x}$ TaqMan master mix (including Taq polymerase)

$0.1 \mu \mathrm{l}$ fwd $(10 \mathrm{pmol} / \mu \mathrm{l})$

$0.1 \mu 1 \mathrm{rev}$ primer $(10 \mathrm{pmol} / \mu \mathrm{l})$

$0.1 \mu 1$ probe

$0.7 \mu 1 \mathrm{H}_{2} \mathrm{O}$

The standard pRT-PCR program was as follows:

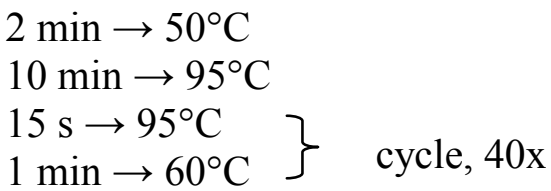

\subsubsection{Statistical analysis or qRT-PCR data}

Ct-values were exported to Excel and normalized to the mean value of one or several reference genes. The obtained $\Delta \Delta \mathrm{Ct}$-values were further de-logarithmized (by using the formula $1 / 1.96^{\mathrm{Ct}}$ ) and normalized to the minimum value. The mean values and standard deviations were calculated.

For complex statistical analyses including two variables such as genotype and time a two-way analysis of variance (ANOVA) was applied with the help of GraphPad Prism 4. ANOVA determines the F-factor, which represents the estimated variances between the groups divided by the estimated variance within the groups. The higher the F-factor, the higher the probability that the difference between samples is due to the treatment and due to the natural variation within the groups. The F-statistic e.g. $\mathrm{F}_{(3,12)}=19.77$ not also calculates the F-factor but provides additional information about the degrees of freedom (n-1) of each variable (indicated in brackets). The $\mathrm{p}$ value is defined as the probability of determining a difference as large as or larger than one would observe if the null hypothesis (no differences between experimental groups) were true.

\subsubsection{Gateway recombination based cloning}

\subsubsection{Standard gateway recombination based cloning}

The gateway recombination system is based on site-directed recombination and uses enzymes derived from the bacteriophage lambda that are able to transfer DNA fragments between vectors (Hartley et al., 2000). This cloning strategy works with much higher efficiency than the conventional cloning technique that is based on type II endonucleases and T4 DNA ligase. For detailed information on gateway cloning see Invitrogen's web page (http://dede.invitrogen.com/site/de/de/home/Products-and-Services/Applications/Cloning/Gateway- 
Cloning.html). To clone an ORF or other DNA fragments of interest specific recombination sites (att $\mathrm{B} 1$ and attB2) have to be added to both ends via PCR. The PCR product was recombined in a 'BP reaction' with a 'donor' plasmid, termed $\mathrm{pDONR}$, containing attP1 and att $\mathrm{P} 2$ recognition sites. The $\mathrm{BP}$ reaction is catalised by the Gateway BP clonase II enzyme mix.

Each BP reaction contained:

$3 \mu \mathrm{lattB-PCR}$ product $(10-100 \mathrm{ng} / \mu \mathrm{l})$

$1 \mu \mathrm{pDONR}(100 \mathrm{ng} / \mu \mathrm{l})$

$1 \mu \mathrm{l}$ BP clonase II

$\mathrm{BP}$ reactions were incubated overnight at RT. To optimize conditions for transformation, $1 \mu 1$ of proteinase $\mathrm{K}$ was added and incubated at $37^{\circ} \mathrm{C}$ for $10 \mathrm{~min}$, followed by a heat-inactivation step at $95^{\circ} \mathrm{C}$ for $10 \mathrm{~min} .1 \mu \mathrm{l}$ of the inactivated $\mathrm{BP}$ reactions was taken for electroporation in $\mathrm{DH} 10 \mathrm{~b}$ bacteria or for chemical transformation in XL1blue bacteria. Clones were selected by the appropriate antibiotic, and the insert was sequenced. One major feature of the gateway system is that bacteria harbouring a non recombined donor vector after a recombination reaction are not supposed to survive as the pDONR vector contains the toxic $c c d B$ gene flanked by the recombination sites. The $c c d B$ gene is only replaced by an insert when the recombination event was successful, ensuring that only properly recombined clones are able to survive and produce colonies. Constructs containing the pDONR backbone and the DNA insert represent 'entry clones' ( $\mathrm{pENTR}$ ). These constructs carry att $\mathrm{L}$ sites (the product of recombination of attB and attP sites). To generate the final 'expression clones', abbreviated $\mathrm{pEXPR}$, the attL sites of the Entry clones are recombined with attR sites of a 'destination vector', or pDEST, in an 'LR' reaction, catalised by the Gateway LR Clonase II enzyme mix.

Each LR reaction included:

$1 \mu 1 \mathrm{pENTR}(25-100 \mathrm{ng} / \mu \mathrm{l})$

$1 \mu \mathrm{l} \operatorname{pDEST}(100 \mathrm{ng} / \mu \mathrm{l})$

$1 \mu 1$ LR clonase

$2 \mu 1 \mathrm{H}_{2} \mathrm{O}$

The reaction was incubated at RT overnight followed by a proteinase $\mathrm{K}$ digest (see above). 1 $\mu 1$ of the reaction was transformed into $\mathrm{DH} 10 \mathrm{~b}$ electro-competent bacteria. The resulting clones pEXPR clones were then functionally tested by transient transfection in cell culture.

\subsubsection{Multisite gateway recombination based cloning}

The multisite gateway pro recombination system from Invitrogen was applied to generate reporter constructs containing three (or more) inserts (Cheo et al., 2004). In this thesis, the Multisite gateway system was used to clone reporter constructs for generating stably transfected Oli-neu cells. The following inserts had to be recombined in a firefly luciferase reporter vector: a promoter region ( $\mathrm{Mal}$ or $\mathrm{Mbp}$, respectively), and two unique expressed oligonucleotide tags (EXTs) reporter to quantify expression (Botvinnik et al., 2010). For detailed description see Invirtogen's webpage (http://dede.invitrogen.com/site/de/de/home/Products-and-Services/Applications/Cloning/GatewayCloning/MultiSite-Gateway-Technology.html). A schematic representation of the threefragment recombination is illustrated in Fig. 27. The technique depends on the high specificity of each att-site to recombine selectively with its designated counterpart. Fragments of interest were amplified by PCR with primers containing the appropriate recombination sites. 
attB1 5'-ggggACAAGTTTGTACAAAAAAGCAGGCTCT - insert specific sequence - 3'

attB4 5'-ggggCAACTTTGTATAGAAAAGTTG - insert specific sequence - 3'

attB4r 5'-ggggCAACTTTTCTATACAAAGTTG - insert specific sequence - 3'

attB3r 5'- ggggCAACTTTATTATACAAAGTTG - insert specific sequence - 3'

attB3 5'-ggggCAACTTTGTATAATAAAGTTG - insert specific sequence - 3'

attB2 5'-ggggACCACTTTGTACAAGAAAGCTGGGTC - insert specific sequence - 3'

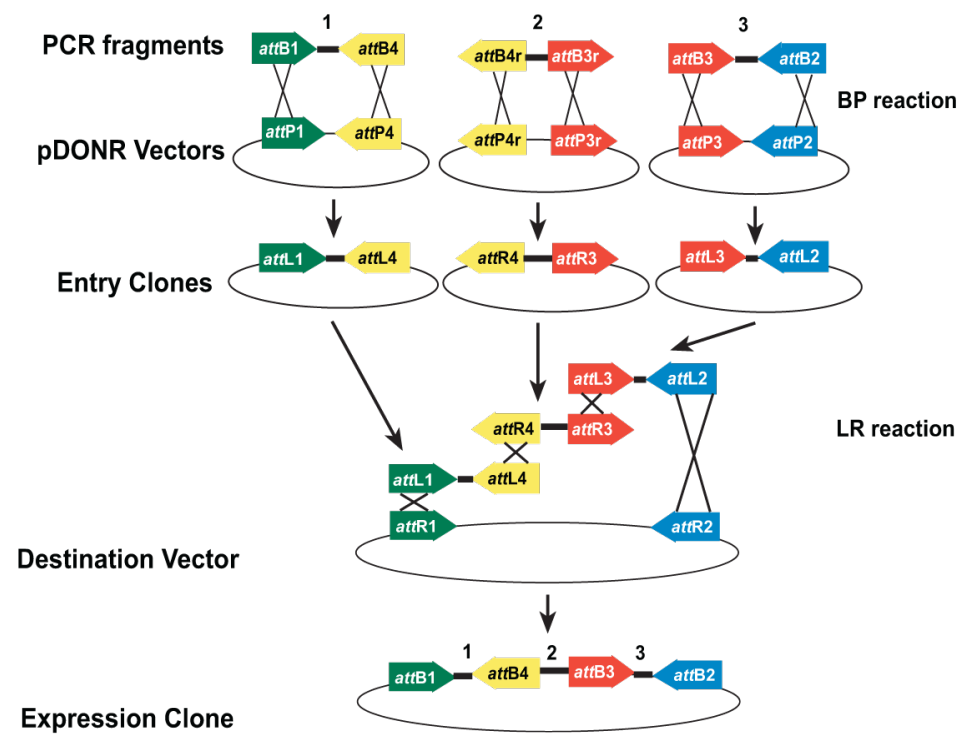

Fig. 27 Schematic representation of the three-fragment recombination cloning

Depending on the desired position of the insert specific att-sites had to be added by PCR. This image is adopted from the Multisite Gateway Pro user manual and illustrates how three fragments can be simultaneously cloned into one expression vector by using multiple specific recombination sites.

In a $\mathrm{BP}$ reaction, corresponding to that of the single fragment gateway, the PCR products were recombined with their corresponding pDONR plasmids:

$3 \mu \mathrm{lattB-PCR}$ product $(10-100 \mathrm{ng} / \mu \mathrm{l})$

$1 \mu \mathrm{pDONR}(100 \mathrm{ng} / \mu \mathrm{l})$

$1 \mu \mathrm{BP}$ clonase II

The following sites were recombined with each other:

$\begin{array}{llllll}\text { PCR product } & \text { (B1-B4) } & - & \text { pDONR (P1-P4) } & - & \text { pENRT (L1-L4) } \\ \text { PCR product } & \text { (B4r-B3r) } & - & \text { pDONR (P4r-P3r) } & - & \text { pENTR (R4-R3) } \\ \text { PCR product } & \text { (B3-B2) } & - & \text { pDONR (P3-P2) } & - & \text { pENTR (L3-L2) }\end{array}$

$\mathrm{BP}$ reactions were incubated overnight at $\mathrm{RT}$ and subjected to a proteinase $\mathrm{K}$ digest as described for the single gateway recombination. $1 \mu \mathrm{l}$ was transformed into DH10b bacteria and positive clones (putative pENTR clones) were selected by the appropriate resistance. The DNA was purified, inserts were checked for correct size by restriction digest and finally sequenced. 
The DNA concentration was determined and entry clones were diluted to a concentration of 5 fmol $/ \mu 1$, destination plasmids to $10 \mathrm{fmol} / \mu \mathrm{l}$. To calculate the corresponding DNA amounts (in nanograms) the following formula was applied:

$$
n g=(f m o l)(N)\left(\frac{660 f g}{f m o l}\right)\left(\frac{1 n g}{10^{6} f g}\right)
$$

With $\mathrm{N}$ being the size of the DNA in base pairs.

Multisite LR reactions were performed as following:

$1 \mu 1 \mathrm{pENTR}$ (L1-L4) $5 \mathrm{fmol}$

$1 \mu \mathrm{pENTR}$ (R4-R3) 5 fmol

$1 \mu 1 \mathrm{pENTR}$ (L3-L2) $5 \mathrm{fmol}$

$1 \mu 1 \mathrm{pDEST}$ (R1-R2) $10 \mathrm{fmol}$

$1 \mu 1$ LR clonase II Plus

The LR reaction was incubated overnight at RT, followed by a proteinase $\mathrm{K}$ digested (see above). $1 \mu \mathrm{l}$ was taken for transformation in DH10b or MACH1 E.coli competent strains. The resulting $\mathrm{pEXPR}$ clones were then functionally tested by transient transfection in cell culture.

\subsection{Cell biology methods}

\subsubsection{Subculturing of eukaryotic cells}

Eukaryotic cells (HEK293, HEK293FT, PC12, Oli-neu) were cultivated on $15 \mathrm{~cm}$ dishes at $37^{\circ} \mathrm{C}$ in a humidified incubator containing $5 \% \mathrm{CO}_{2}$. After reaching 70-90\% confluency, cells were subcultured by washing with PBS and mild treatment with $2.5 \mathrm{ml}$ trypsin/EDTA for 1-5 min at $37^{\circ} \mathrm{C}$ until they detached from the culture dish. The reaction was stopped by adding 5 $\mathrm{ml}$ serum-containing culture medium, and the cells were pelleted by centrifugation for 5 min at $800 \mathrm{~g}$. The supernatant was discarded, the pellet resuspended in fresh medium, and an appropriate inoculum (typically, a dilution of 1/10 to 1/5) was plated onto new dishes.

\subsubsection{Thawing and cryopreservation of cell lines}

Frozen stocks of cell lines were kept at $-196^{\circ} \mathrm{C}$ submerged in liquid nitrogen in $2 \mathrm{ml}$ cryovials. Aliquots were quickly thawed in a water bath at $37^{\circ} \mathrm{C}$, suspended in $30 \mathrm{ml}$ warm growth medium, and centrifuged for $5 \mathrm{~min}$ at $800 \mathrm{~g}$ to remove the DMSO. The pellet was resuspended in fresh medium and plated onto a $10 \mathrm{~cm}$ or $15 \mathrm{~cm}$ culture dish.

For cryopreservation cells were grown on $15 \mathrm{~cm}$ dishes until reaching $90 \%$ confluency. The medium was removed, and the cells were detached using trypsin as described above. The cells were counted using a Neubauer chamber, and, after centrifugation, resuspended in an appropriate volume of ice-cold growth medium to obtain a concentration of about 2 million cells per $1 \mathrm{ml} .500 \mu \mathrm{l}$ of the cell suspension was transferred into a cryovial already containing an equal volume of the ice-cold $2 \mathrm{x}$ freezing medium. The samples were mixed gently by inverting the tubes three times and quickly frozen at $-20^{\circ} \mathrm{C}$. After two hours the cells were transferred to $-80^{\circ} \mathrm{C}$ for one day and finally to liquid nitrogen for long term storage. 


\subsubsection{Coating with poly-L-lysin}

For PC12 and Oli-neu cells, it is important to coat the dishes with poly-L-lysin (PLL). The negatively charged polysaccharid structures on the cell surface bind to the positively charged PLL allowing better adherence of the cells. For coating, dishes were incubated with 0.02 $\mathrm{mg} / \mathrm{ml}$ (permanent cell lines) or $0.1 \mathrm{mg} / \mathrm{ml}$ (for primary cultures) $\mathrm{PLL}$ in $\mathrm{ddH}_{2} \mathrm{O}$ for $20 \mathrm{~min}$ at $\mathrm{RT}$, then washed twice with sterile water, air-dried, and stored at $4^{\circ} \mathrm{C}$ until further use.

\subsubsection{Transfection of mammalian cells}

\subsubsection{DNA transfer in eukaryotic cells with lipofectamine2000}

The transient transfection of plasmid DNA in eukaryotic cells was done using lipofectamine2000 (Invitrogen). The approach is based on the formation DNA-containing liposomes that are endocytosed by the cell. Cells were typically transfected in 96-well plates for reporter gene assays. Depending on the cell line different numbers of cells were plated:

$\begin{array}{ll}\text { Cell line } & \text { 96-well } \\ \text { PC12 WT } & 50000 \\ \text { HEK293 } & 20000 \\ \text { HEK293FT } & 20000 \\ \text { Oli-neu } & 5000\end{array}$

The protocol provided by the manufacturer was modified to obtain optimal results. For a single well of a 96-well plate, both 10-200 ng (mini or midi preparation) of DNA and $0.2 \mu 1$ of lipofactamine2000 were diluted in $15 \mu$ OptiMEM each. The solutions were mixed separately, incubated for $5 \mathrm{~min}$, and then combined, resulting in $30 \mu \mathrm{l}$. The mix was vortexed and incubated for $20 \mathrm{~min}$ at RT. The growth medium was completely removed from the cells, and $30 \mu \mathrm{l}$ of the OptiMEM containing DNA/lipofectamine complexes were applied onto the cells. After two hours, $30 \mu \mathrm{l}$ of regular growth medium was added to each well. The cells were allowed to express the plasmids for $24-48 \mathrm{~h}$, and subjected to a given procedure, i.e. western blots or reporter gene assays.

\subsubsection{DNA transfer in eukaryotic cells with electroporation}

During electroporation a brief current pulse induces a transmembrane electric field in the cells which leads to local instabilites of the cell membrane (electro-pores). In this short period macromolecules such as DNA are able to enter the cell. With optimal setting of the parameters, cells recover and can be subcultured. For the generation of stably transfected Olineu cells, $10^{6}$ cells were mixed with $20 \mu \mathrm{g}$ plasmid and transferred into an electroporation cuvette $(4 \mathrm{~mm})$.

Parameters for electroporation were the following: $950 \mu \mathrm{F}, 220 \mathrm{~V}$.

After electroporation, cells were incubated under standard conditions.

\subsubsection{Nucleofection of primary cultured oligodendrocytes with Amaxa}

Nulceofection of primary cultured oligodendrocytes was performed with the Amaxa nucleofector device using the 'mouse neuron nucleofector kit' provided by Amaxa Biosystems. Oligodendrocyte precursor cells (OPCs) were harvested from mixed glial cultures by shaking (30x) and pelleted by centrifugation for $10 \mathrm{~min}$ at $800 \mathrm{~g}$ at RT. Cells were 
resuspended in 1-2 $\mathrm{ml}$ of a modified Sato medium and counted using a Neubauer chamber. For each electroporation experiment, 750000 cells were pelleted and resuspended in $100 \mu 1$ equilibrated basic nucleofector solution ('for primary neurons'). The DNA was added to the cells and the mix was transferred to the electroporation cuvette. Electroporation was performed according to the 0-005 program. $500 \mu \mathrm{l}$ of RPMI medium (Gibco) without supplements was quickly added and the cells were allowed to recover for 2-5 min. Subsequently, the cells were transferred to a $15 \mathrm{ml}$ falcon tube and $2.5 \mathrm{ml}$ of a modified Sato medium was added. $100 \mu 1$ of the cell suspension was plated per well into a PLL $(0.1 \mathrm{mg} / \mathrm{ml})$ coated 96-well plate. The cells were allowed to grow and differentiate for four days. Transfection efficiency was documented by fluorescence microscopy. For luciferase reporter gene assays, sample preparation and measurement were performed as described below with the exception that only the firefly luciferase activity was measured. For data analysis, means and standard deviations were calculated for 24 technical replicates. The data were normalized to the mock control values. Statistical analysis was performed according to the Mann-Whitney test.

\subsubsection{Luciferase reporter gene assays}

Luciferases are enzymes which catalytic activity leads to the emission of visible light upon substrate conversion. The most commonly used luciferase is the firefly luciferase that catalyses the following reaction:

luciferin $+\mathrm{ATP} \rightarrow$ luciferyl adenylate $+\mathrm{PP}_{\mathrm{i}}$

luciferyl adenylate $+\mathrm{O}_{2} \rightarrow$ oxyluciferin $+\mathrm{AMP}+$ light

Transcriptional activity of a given promoter can be investigated by transfecting the cells with a reporter construct carrying a luciferase ORF under the control of this particular promoter. The expression level of the luciferase reporter gene is then quantified by adding luciferase substrate and ATP to the cellular lysates and measuring the intensity of the emitted light.

Luciferase reporter gene assays were performed in HEK293 cells, PC12 cells, Oli-neu cells, as well as primary mouse neurons and oligodendrocytes in 96-well plates. 24-48 h after transfection the medium was removed and $30 \mu \mathrm{l}$ of $1 \mathrm{x}$ passive lysis buffer (Promega) was added to each well. The plates were incubated for $20 \mathrm{~min}$ at RT under moderate shaking (200 $\mathrm{rpm}$ ) and assayed immediately or frozen at $-20^{\circ} \mathrm{C}$. Prior to analysis the samples were transferred to black plastic microtiter plates to minimise light signal leakage and cross-mixing because of light reflection.

Measurements of the firefly luciferase activity are commonly normalized to the activity of a different reporter, e.g. renilla luciferase which is expressed under a constitutively active promoter. This is done to rule out differences in cell number, transfection efficiency, and RNA and protein expression from well to well. A mix of three different plasmids was carrying the SV40, TK or CMV promoter to express the renilla luciferase (renilla mix). The plasmids were mixed at the following ratio: SV40:TK:CMV $=10: 2: 1$ to compensate for the different potency of the promoters. $20 \mathrm{ng}$ of the renilla mix was cotransfected per well along with any firefly reporter gene assay, if not indicated otherwise. To visually control transfection efficiency an equal amount of pEYFPnuc, a construct for CMV promoter-driven expression of the nuclear localized enhanced yellow flourescent protein was used. For standard luciferase reporter gene assays six technical replicates were analysed to enhance statistical reliability. Bioliminescence measurement was performed with the help of the Microplate reader Mitras LB940 (Berthold Technologies) and the associated software MicroWin 2000 using the following protocol: 
1) Injection of $75 \mu \mathrm{l}$ firefly luciferase substrate, $2 \mathrm{~s}$ reaction time, $10 \mathrm{~s}$ measurement of light signals

2) Injection of $75 \mu \mathrm{l}$ renilla luciferase substrate, $2 \mathrm{~s}$ reaction time, $10 \mathrm{~s}$ measurement of light signals

The firefly and the renilla luciferases display high substrate specificity and do not cross-react. Moreover, firefly luciferase activity is blocked by the $\mathrm{pH}$ conditions of the renilla substrate.

Statistical analysis

Data were exported from MicroWin 2000 and analyzed with Excel. For normalization the firefly-value was divided by the corresponding renilla-value to get the relative luciferase unit (RLU). Mean and standard deviation (SD) of the six technical replicates were calculated as well as the level of significance according to the Mann-Whitney test.

\subsubsection{Preparation of primary mouse oligodendrocytes}

The brains of newborn mice aged P0 were prepared (always three at once) and stored in a dish containing prewarmed HBSS+. Under a binocular the cerebelli, bulbi olfactorii and meninges were carefully removed. The tissue was transferred to $15 \mathrm{ml}$ falcon tubes and further stored in $\mathrm{HBSS}+$ at $37^{\circ} \mathrm{C}$. The liquid was removed and $1 \mathrm{ml}$ per brain trypsin/EDTA (f.c. $0.5 \% / 0.1 \%$ in BME medium) was added. First, the samples were softly triturated with a $5 \mathrm{ml}$ glass pipet for 5 times and then digested for $10 \mathrm{~min}$ at $37^{\circ} \mathrm{C}$. The reaction was stopped by adding an equal volume of BME medium. All liquid was removed and fresh BME medium was added $(1 \mathrm{ml}$ per brain). The suspension was transferred to PLL-coated $(0.1 \mathrm{mg} / \mathrm{ml})$ cell culture flasks containing $10 \mathrm{ml}$ prewarmed and equilibrated BME medium each. The suspension was divided into $1 \mathrm{ml}$ aliquots corresponding to 1 brain. Each aliquot was transferred into one flask. The mixed glial cultures were cultivated at $37^{\circ} \mathrm{C}$ and $5 \% \mathrm{CO}_{2}$ for $10-14$ days. Every 3-4 days the medium was exchanged. Usually within one week an astrocyte layer was formed with oligodendrocyte precursor cells (OPCs) growing on top of it. Prior to harvesting the OPCs, microglia were removed by careful shaking. Afterwards, the OPCs were enriched in the supernatant by shaking the cell culture flasks 30 times. The OPCs were pelleted by centrifugation and counted using a Neubauer chamber. Usually, 20000-30000 cells were plated per well in a 24-well format previously coated with PLL $(0.1 \mathrm{mg} / \mathrm{ml})$ in a modified Sato medium. Within five-seven days culturing in vitro most oligodendrocytes had differentiated into a myelin basic protein (MBP)-positive stage.

\subsubsection{Preparation of primary mouse neurons}

The brains of mouse embryos on embryonal day (E) 17 were dissected and stored in ice-cold HBSS+. Meninges were carefully removed and cortices were isolated under a binocular. The neural tissue was transferred into $15 \mathrm{ml}$ falcons and digested with trypsin/EDTA (f.c. $0.5 \% / 0.1 \%$ in BME medium) and triturated with a Pasteur pipet (one time). After addition of DNaseI solution $(0.05 \%$ in HBSS + ), the brains were triturated once again, followed by an incubation step for $10 \mathrm{~min}$ at RT. The digest was terminated with $10 \%$ HS and cells were washed twice with HBSS + . In between, cells were pelleted by centrifugation for $10 \mathrm{~min}$ at $800 \mathrm{rpm}$. Finally, the tissue was triturated again with a normal Pasteur pipet (one time), followed by a narrowed Pasteur pipet (five times). The cells were washed with HBSS + and resuspended in 1-2 $\mathrm{ml}$ Neurobasal medium (including additives). The cell number was determined using a Neubauer chamber and neurons were plated in PLL-coated $(0.1 \mathrm{mg} / \mathrm{ml})$ 96-well plates (80000 cells per well) for luciferase reporter gene assays. 


\subsection{Generation of stably transfected Oli-neu cell lines}

To generate stably transfected Oli-neu cell lines, reporter constructs were cloned with the help of the multisite gateway recombination system by Invitrogen. The plasmids contained the following cassettes: the proximal promoter regions of $\mathrm{Mal}$ or $\mathrm{Mbp}$ respectively, and two EXTs to monitor transcription (Botvinnik et al., 2010). These inserts were recombined into a promoter-less modified pGL4 vector (Invitrogen) down-stream of the firefly luciferase reporter gene, containing a hygromycin resistance cassette which allows for selection in eukaryotic cells (Fig. 28).

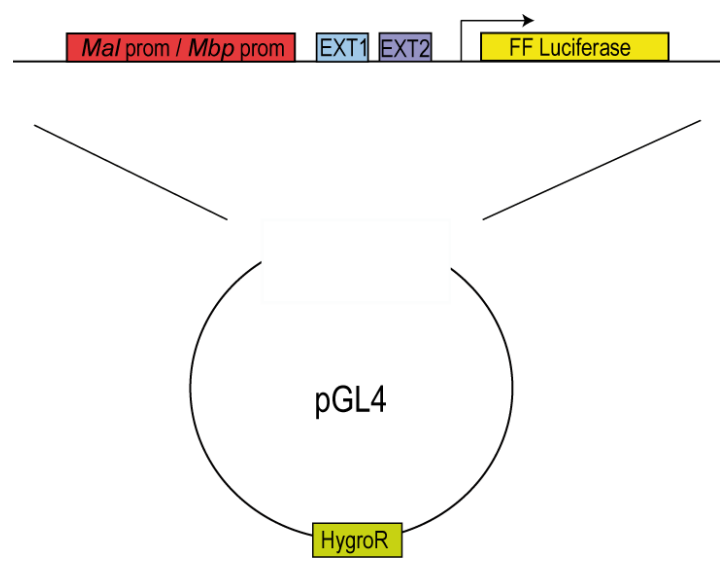

Fig. 28 Schematic representation of the pEXPR Mal/Mbp prom EXT (GL4) reporter constructs Prom, promoter; EXT, expressed unique sequence tag; FF, firefly; HygroR, hygromycin resistance.

Prior to electroporation, the appropriate hygromycin concentration for selection of positive clones had to be determined. Therefore, a 'killing curve' with WT Oli-neu cells was performed in which antibiotic concentrations in the range from $0-425 \mu \mathrm{g} / \mathrm{ml}$ hygromycin were tested. The following rule was applied to find the proper concentration: after appr. five days $50 \%$ of the cells should be dead. $225 \mu \mathrm{g} / \mathrm{ml}$ hygromycin turned out to fit this criterium.

For electroporation, $10^{6}$ Oli-neu cells were mixed with $20 \mu \mathrm{g}$ plasmid DNA pEXPR Mal prom EXT (GL4) or pEXPR Mbp prom EXT (GL4) and transferred into an electroporation cuvette $(0.4 \mathrm{~mm})$.

Parameters for electroporation were the following: $950 \mu \mathrm{F}, 220 \mathrm{~V}$.

After two days of recovering selection with hygromycin was started. The medium was exchanged every other day. Immediately after the appearance of visible cell clones on the dishes these were picked with a pipet and separately cultivated for further analysis.

\subsection{Generation of recombinant lentiviruses}

To produce SHARP2 expressing lentiviruses $3 \times 10^{6}$ HEK293FT cells were plated on PLL $(0.02 \mathrm{mg} / \mathrm{ml})$ coated $15 \mathrm{~cm}$ cell culture dishes in HEK medium without antibiotics. The cells were allowed to grow overnight in a humidified incubator at $37^{\circ} \mathrm{C}$ and $5 \% \mathrm{CO}_{2}$. On the next day, medium was replaced by $15 \mathrm{ml}$ of fresh HEK medium without antibiotics. For transfection, $15 \mu \mathrm{g}$ pLenti SHARP2 transfer plasmid (plus $1 \mu \mathrm{g}$ pLenti EYFP as infection control), $12 \mu \mathrm{g}$ packaging plasmid psPAX2 and $4 \mu \mathrm{g}$ envelope plasmid pMD2.G were mixed with $500 \mu \mathrm{l}$ OptiMEM and vortexed briefly. $120 \mu 1$ polyethylenimine (PEI) transfection reagent was added to reach the final concentration of $1 \mu \mathrm{g} / \mu \mathrm{l}$. The mixture was vortexed for $10 \mathrm{~s}$ and incubated for $10 \mathrm{~min}$ at RT. Finally, the DNA/OptiMEM/PEI mix was added drop- 
wise to the cells. After six hours of incubation, the medium was replaced by $15 \mathrm{ml}$ of fresh HEK medium without antibiotics. Sodium butyrate at a final concentration of $10 \mathrm{mM}$ was added to ensure cell cycle arrest. 48 hours post transfection, the cell supernatant was collected to harvest the virus particles. First, cell debris was removed by centrifugation for $5 \mathrm{~min}$ at $1000 \mathrm{rpm}$ at $4{ }^{\circ} \mathrm{C}$ and followed by filtration $(0.45 \mu \mathrm{m}$ pore size $)$. To concentrate the lentivirus an Amicon ultra-15 centrifugal filter unit (Millipore) was applied. After an additional centrifugation step at $3500 \mathrm{rpm}$ for $15 \mathrm{~min}$ at $4^{\circ} \mathrm{C}$ the viruses were washed twice with $10 \mathrm{ml}$ Sato medium containing 1\% horse serum (the same medium was then used to cultivate and infect primary oligodendrocytes). After a third and last centrifugation step, virus particles were resuspended in a proper amount of infection medium depending on the desired titer. The virus could be stored at $4^{\circ} \mathrm{C}$ for up to one week.

\subsubsection{Titration of lentiviruses by qRT-PCR}

For determination of the viral titer, viral RNA was quantified by qRT-PCR. First, $5 \mu l$ of the concentrated lentiviral sample DNA were diluted with $22 \mu 1 \mathrm{H}_{2} \mathrm{O}$ and subjected to an DNaseI digest by adding $3 \mu 1 \mathrm{DNaseI}$ buffer and $1 \mu \mathrm{LNaseI}(1 \mathrm{U} / \mu \mathrm{l})$. The reaction was incubated for $30 \mathrm{~min}$ at $37^{\circ} \mathrm{C}$ and terminated by adding $3 \mu \mathrm{l}$ Stop-solution (Qiagen) and incubation for 10 min at $75^{\circ} \mathrm{C}$. This step also causes the release of the viral RNA genome. The viral RNA was subsequently reverse transcribed into cDNA following the standard cDNA synthesis protocol (using SuperscriptIII).

Each SYBR Green lentivirus titration assay contained $5 \mu 12 x$ SYBR Green master mix, $1 \mu 1$ of each forward (\#22945) and reverse primer (\#22946) $(10 \mathrm{pmol} / \mu \mathrm{l}), 1 \mu \mathrm{l} \mathrm{ddH}_{2} \mathrm{O}$ and $4 \mu \mathrm{l}$ cDNA sample (diluted 1:10, 1:000 and 1:1000). For absolute quantification of the viral RNA, a linear dilution of a pLenti6 EXPR plasmid in the range from $10^{\circ}$ to $10^{6}$ molecules as well as a no template control was co-amplified. Two technical controls were performed. The standard qRT-PCR protocol described above was applied. The qRT-PCR was performed in specially designated 96-well plates (Applied Biosystems) using the Fast Real-Time-PCR System (Applied Biosystems).

The standard curve for the linear pLenti6 EXPR dilution series was determined and the viral titer $(\mathrm{x})$ could be calculated using the following formula:

$y=m * \operatorname{Ln}(x)+n$

$\mathrm{m}=$ slope of standard curve

$\mathrm{n}=$ intersection point with $\mathrm{y}$-achsis

The viral titer was determined by the following equation:

Copies $/ \mathrm{ml}=[\mathrm{x} * 1000 \mu \mathrm{l} / \mathrm{ml} * \mathrm{a} * \mathrm{~b}] /[\mathrm{c} * \mathrm{~d}]$

$\mathrm{a}=$ volume of $\mathrm{cDNA}$ synthesis reaction

$\mathrm{b}=$ dilution of cDNA for qRT-PCR (e.g. 10, 100, 1000)

$\mathrm{c}=$ volume of viral supernatant in cDNA synthesis reaction

$\mathrm{d}=$ volume of cDNA input into $\mathrm{qRT}-\mathrm{PCR}$ 


\subsubsection{Infection of stably transfected Oli-neu cell lines}

150000 Oli-neu cells stably expressing the firefly luciferase reporter gene under the control of either the $1.5 \mathrm{~kb} \mathrm{Mal}$ promoter region or the $1.3 \mathrm{~kb} \mathrm{Mbp}$ promoter region were plated on PLL $(0.02 \mathrm{mg} / \mathrm{ml})$ coated $6 \mathrm{~cm}$ dishes and infected with the appropriate viral titer with a multiplicity of infection (MOI) of 10 into the growth medium. The MOI is the ratio of the infectious virus particles to cells. After 72 hours, Oli-neu cells were plated into a 96-well plate and further incubated for 24 hours. Infection efficiency was documented by fluorescence microscopy. Sample preparation and luciferase reporter gene assays were performed as described before with the exception that only the firefly luciferase activity was measured. For data analysis, means \pm SD were calculated for $n=24$ technical replicates. The data were normalized to the signal of the mock control value and plotted as expressed as fold induction. Statistical analysis was performed with the Mann-Whitney test.

\subsection{Protein biochemistry methods}

\subsubsection{Preparation of protein extracts from mouse tissue}

Wild type (WT) C57/B16 and SHARP1 and -2 double null-mutant $\left(\mathrm{S} 1 / 2^{-/-}\right)$mice were sacrified at different circadian time points, zeitgeber time (ZT) 4, 10, 16 and 22. The brains were placed in a 'rodent brain matrix' $1 \mathrm{~mm}$ coronal slicer (ASI Instruments, Warren, MI) to isolate the prefrontal cortex and corpus callosum from Bregma $-0.8 \mathrm{~mm}-1.18 \mathrm{~mm}$. Three mice per genotype and time point were analysed. After preparation, the samples were immediately frozen on dry ice. The tissue was stored in eppendorf tubes at $-80^{\circ} \mathrm{C}$. For further analysis, corresponding samples were pooled.

To prepare protein extracts sucrose buffer was added in adequate amounts (500 $\mu 1$ for the corpus callosum, $1500 \mu 1$ for the cortex) to the frozen tissue. The samples were immediately homogenized with the help of the Precellys homogenizer and ceramic beads ( 25 beads per sample, $1.4 \mathrm{~mm}$ diameter) 2 times for $10 \mathrm{~s}$ at $5500 \mathrm{rpm}$.

\subsubsection{Determination of protein concentration}

Protein concentration determination was performed by using the DC Protein Assay (Bio-Rad), which is based on the same principles as the Lowry assay (LOWRY et al., 1951). The analysis was performed in a microtiter plate according to the manufacturer's instructions. In brief, a standard BSA series was prepared ranging from $0.2 \mathrm{mg} / \mathrm{ml}$ to $1.5 \mathrm{mg} / \mathrm{ml}$ protein. In parallel, appropriate dilutions of the samples were made (usually 1:5). $5 \mu 1$ of standards and samples were pipeted into a microtiter plate with at least three technical replicates. $25 \mu 1$ of reagent A was added following by $200 \mu \mathrm{l}$ of reagent B. After $15 \mathrm{~min}$ absorbance was read at $750 \mathrm{~nm}$ in a microplate reader (Mithras LB 940, Berthold Technologies).

\subsubsection{Western Blot}

Western blotting is a classical method for immunological detection of proteins in biological samples (Towbin et al., 1979). 


\subsubsection{Sodium dodecyl sulphate polyacrylamid gel electrophoresis (SDS-PAGE)}

Unlike DNA, proteins may be positively or negatively charged or neutral and do not have a linear relationship between weight and charge due to differences in amino acid composition. To enable directed migration of proteins in the electric field SDS is used to produce a negative charge. After heat denaturation at $95^{\circ} \mathrm{C}$ the polypeptide chains unfold to expose the hydrophobic regions which can non covalently bind SDS hydrophobic tails at the stochiometry of 1 SDS molecule per 2 amino acids. Additional treatment with dithiotreitol (DTT) reduces all intra- and intermolecular disulfide bonds.

Protein lysates were mixed with 4x LDS sample buffer and DTT (f.c. $0.1 \mathrm{M}$ ) and were incubated at $70^{\circ} \mathrm{C}$ for $10 \mathrm{~min} .5 \mu \mathrm{g}$ were used for western blotting. For SDS-PAGE NuPAGE Novex Bis-Tris Gels (Invirtogen) were used with Xcell SureLock NuPAGE buffer chambers (Invitrogen) according to the manufacturer's protocol. The electrophoresis was performed at $200 \mathrm{~V}$ for $45-60 \mathrm{~min}$.

\subsubsection{Transfer}

The transfer of proteins from the gel to a PVDF membrane was performed with the Xcell SureLock Western Blot System (Invitrogen) according to the manufacturer's instructions. Prior to transfer PVDF membranes were activated for $10 \mathrm{~min}$ in $100 \%$ methanol and afterwards rinsed in transfer buffer. The transfer was done at $30 \mathrm{~V}$ for $2 \mathrm{~h}$ while the chamber was placed in ice-water.

\subsubsection{Detection}

When the transfer was completed the membrane was incubated in blocking buffer $(5 \%$ non fat milk powder in TBS-T) for $30 \mathrm{~min}$ at RT. The primary antibody was applied in the appropriate dilution in blocking buffer at $4{ }^{\circ} \mathrm{C}$ overnight. The membrane was washed with TBS-T three times for $10 \mathrm{~min}$ und was subsequently incubated with the appropriate horse radish peroxidase (HRP)-conjugated secondary antibody for $1 \mathrm{~h}$ at RT. After three wash steps for $10 \mathrm{~min}$ in TBS-T the membrane was treated with enhanced chemiluminescence (ECL) detection solution for $1 \mathrm{~min}$ according to the manufacturer's recommendations and enclosed in plastic foil. In a dark chamber an ECL-hyperfilm was exposed to the membrane and developed with KODAK-O-MAT. For reprobing the membrane was incubated with stripping buffer for $1 \mathrm{~h}$ at $55^{\circ} \mathrm{C}$. The membrane was then washed in TBS-T and blocked again as described above. All incubation steps were performed under constant moderate shaking.

\begin{tabular}{|ll|}
\hline Primary antibody & Dilution \\
$\alpha$-CNP, monoclonal mouse & $1: 10000$ \\
$\alpha$-MAL, polyclonal rabbit & $1: 500$ \\
$\alpha$-MBP, polyclonal rabbit & $1: 5000$ \\
$\alpha$-PLP, polyclonal rabbit & $1: 5000$ \\
$\alpha$-alpha-Tubulin, monoclonal mouse & $1: 20000$ \\
\hline \multicolumn{2}{|l}{} \\
\hline Secondary antibody & Dilution \\
HRP-goat- $\alpha$-mouse & $1: 10000$ \\
HRP-goat- $\alpha$-rabbit & $1: 10000$ \\
\hline
\end{tabular}




\subsection{Histological methods}

\subsubsection{Preparation of paraffin embedded sections}

The tissue was infiltrated with paraffin using a tissue processor (Microm HMP 110) and the following program:

\begin{tabular}{|ll|}
\hline Reagent & duration \\
$50 \%$ Ethanol & $1 \mathrm{~h}$ \\
$70 \%$ Ethanol & $2 \mathrm{~h}$ \\
$70 \%$ Ethanol & $2 \mathrm{~h}$ \\
$96 \%$ Ethanol & $1 \mathrm{~h}$ \\
$96 \%$ Ethanol & $1 \mathrm{~h}$ \\
$100 \%$ Ethanol & $1 \mathrm{~h}$ \\
$100 \%$ Ethanol & $1 \mathrm{~h}$ \\
2-Propanol & $1 \mathrm{~h}$ \\
Xylol & $2 \mathrm{~h}$ \\
Xylol & $2 \mathrm{~h}$ \\
Paraffin & $2 \mathrm{~h}$ \\
Paraffin & $2 \mathrm{~h}$ \\
\hline
\end{tabular}

Afterwards the material was embedded into liquid heated paraffin with the help of an embedding machine (Microm AP 280) and the blocks were allowed to solidify on a cold plate. Sections of $5 \mu \mathrm{m}$ thickness were made with a sliding microtom (Microm HM 400), transferred to a heated water bath $\left(40^{\circ} \mathrm{C}\right)$ and mounted on glass slides (Marienfeld), which were then dried at $37^{\circ} \mathrm{C}$ overnight.

\subsubsection{DAB staining of paraffin embedded tissue}

The DAB staining depends on the enzymatic reaction of the HRP with the 3-3'diaminobenzidin (DAB) substrate.

Paraffin embedded sections first have to be deparaffinated and rehydrated prior to staining. The sections were heated to $60^{\circ} \mathrm{C}$ for $10 \mathrm{~min}$ followed by $2 \mathrm{x} 10 \mathrm{~min}$ incubation in xylol and $10 \mathrm{~min}$ in xylol/2-propanol (1:1) to remove the paraffin. The sections further were rehydrated by descending alcohol dilutions $(100 \%, 90 \%, 70 \%$ and $50 \%)$ for $5 \mathrm{~min}$ each and finally placed into $\mathrm{ddH}_{2} \mathrm{O}$. For antigen retrieval the slides were incubated in citrate buffer for 5 min at RT and then boiled in the same buffer for $10 \mathrm{~min}$ in the microwave. After cooling down the sections were washed in Tris buffer supplemented with $2 \%$ non fat milk powder for 5 min before they were mounted to the Coverslip System (Dako). Washing buffer was applied to ensure the correct positioning of the coverslips. The endogenous peroxidases in the tissue were inactivated by incubation in $100 \mu 13 \% \mathrm{H}_{2} \mathrm{O}_{2}$ for $5 \mathrm{~min}$. The sections were again washed and then blocked for 10 min with goat serum (1:5 diluted in PBS/BSA) to reduce unspecific background staining. $100 \mu 1$ of the primary antibody, diluted in PBS/BSA, was applied overnight at $4^{\circ} \mathrm{C}$. On the following day the sections were washed with washing buffer and then incubated for $10 \mathrm{~min}$ at RT in the biotinylated anti-mouse and anti-rabbit secondary antibody ( $\mathrm{LSAB}_{2}$ System, DAKO). After washing, $100 \mu \mathrm{l}$ of streptavidine-conjugated HRP $\left(\mathrm{LSAB}_{2}\right.$ System, DAKO) was added for 10 min. Finally, the sections were rinsed with Tris buffer and removed from the coverslips to proceed with the staining. The tissue was encircled by the water-repelling Cytomation Pen (DAKO), which establishes a hydrophobic barrier 
around the tissue. By application of $100 \mu$ DAB substrate solution the enzyme-based histochemical reaction took place. After 10 min the sections were rinsed $2 \mathrm{x}$ in $\mathrm{ddH}_{2} \mathrm{O}$ for 10 min and counterstained with hemalaun for $30 \mathrm{~s}$, briefly incubated in $\mathrm{HCl}$-alcohol and placed in Scott's solution. For dehydration, an ascending series of ethanol dilutions $(50 \%, 70 \%, 90 \%$ and $100 \%)$ was used. The sections were incubated for $10 \mathrm{~min}$ in xylol/2-propanol (1:1) and $2 \mathrm{x}$ $10 \mathrm{~min}$ in xylol and finally mounted with the xylol-based medium Eukitt.

\begin{tabular}{|ll|}
\hline Primary antibody & Dilution \\
$\alpha-\mathrm{CNP}$, monoclonal mouse & $1: 150$ \\
\hline
\end{tabular}

\subsubsection{Immunocytochemistry}

In order to characterise primary cultured oligodendrocytes, immunocytochemistry and fluorescent microscopy were performed. Therefore, cells were grown on coverslips in a 24well plate. Prior to staining, cultures were fixed with 4\% PFA for $10 \mathrm{~min}$ at RT and washed three times with PBS. Coverslips were carefully removed from the cell culture plate and placed on parafilm. The cells were permeabilized with $100 \mu 10.1 \%$ Triton in PBS for 1 min. After three washing steps with PBS $100 \mu$ blocking buffer (2\% FCS, 2\% BSA, 2\% fish gelantine in PBS) was applied for 1 hour at RT. Subsequently the blocking buffer was replaced by the primary antibody in its appropriate dilution in $10 \%$ blocking buffer in PBS. For best results, the incubation was performed at $4{ }^{\circ} \mathrm{C}$ overnight in a humidified chamber. On the next day, after three washing steps with PBS, the secondary antibody (in its appropriate dilution in $10 \%$ blocking buffer in PBS) was applied for 45 min RT. Optionally, DAPI (f.c. $0.5 \mu \mathrm{g} / \mathrm{ml}$ ) was added to the secondary antibody to counterstain cell nuclei. Again, cells were washed with PBS for three times. To remove salts, the coverslips were shortly dipped in $\mathrm{H}_{2} \mathrm{O}$ and mounted in PolyAquaMount.

\begin{tabular}{|ll|}
\hline Primary antibody & Dilution \\
$\alpha$-GFAP, monoclonal mouse & $1: 250$ \\
$\alpha$-MBP, polyclonal rabbit & $1: 250$ \\
$\alpha$-OLIG2, polyclonal rabbit & $1: 200$ \\
\hline \multicolumn{2}{|l}{} \\
\hline Secondary antibody & Dilution \\
Cy2-goat- $\alpha$-mouse & $1: 500$ \\
Cy3-goat- $\alpha$-rabbit & $1: 2000$ \\
\hline
\end{tabular}

\subsubsection{Preparation and dehydration of cryosections for LCM}

$\mathrm{WT}$ and $\mathrm{S} 1 / 2^{-/-}$mice were sacrificed at four months of age by cervical dislocation at zeitgeber time (ZT) $4,10,16$, and 22 with $\mathrm{n}=3$ mice per genotype and time point. Brains were freshly dissected and immediately frozen on dry ice, wrapped in aluminium foil and parafilm and stored at $-80^{\circ} \mathrm{C}$ until sectioning. Cryosections were performed using the cryostat Cryocut CM3000 Jung (Leica). First, tissues were placed in the cryostat and allowed to adjust the temperature for appr. $1 \mathrm{~h}$. With the help of Shandon Cryomatrix (Thermo Electron Corporation) the brains were fixed on metal cutting templates. Serial coronal $14 \mu \mathrm{m}$ thick brain sections were prepared from the left hemisphere at Bregma $-0.7 \mathrm{~mm}$. For subsequent laser-captured microdissection (LCM) the sections were mounted onto PEN membrane frame slides (Molecular devices), and kept at $-80^{\circ} \mathrm{C}$ until dehydration. 
For dehydration, sections were quickly thawn for $5 \mathrm{~s}$ under an $\mathrm{N}_{2}$ stream and incubated in the following solutions:

\begin{tabular}{|ll|}
\hline $70 \% \mathrm{EtOH}$ & $30 \mathrm{~s}$ \\
$70 \% \mathrm{EtOH}$ & $30 \mathrm{~s}$ \\
$95 \% \mathrm{EtOH}$ & $30 \mathrm{~s}$ \\
$100 \% \mathrm{EtOH}$ & $60 \mathrm{~s}$ \\
$100 \% \mathrm{EtOH}$ & $60 \mathrm{~s}$ \\
Xylol & $60 \mathrm{~s}$ \\
Xylol & $60 \mathrm{~s}$ \\
\hline
\end{tabular}

Afterwards, slides were air-dried and kept in a box containing dissicant. On the same day, LCM was performed.

\subsection{Laser-captured microdissection}

Laser-captured microdissection was done using the Veritas Microdissection System (Arcturus). Precise micro-areas of the cortex and corpus callosum such as the anterior cingulate cortex (ACC), the somatosensory motor cortex (SSMCx) and the corpus callosum (CC) micro-area were dissected using the UV laser and attached to the CapSure LCM MacroCaps with the help of the infrared laser. 12 micro-areas for the ACC, 6 for the SSMCX and 24 for the CC were collected separately on caps. The caps were then placed on $0.5 \mathrm{ml}$ Eppendorf tubes containing $350 \mu \mathrm{l}$ RLT buffer. The tubes were incubated upside down for 5 min to ensure lysis of the tissue, vortexed thoroughly and frozen on $-80^{\circ} \mathrm{C}$ until further analysis.

\subsection{Production and maintenance and transgenic mice}

\subsubsection{Used mouse strains}

All experiments described in this work were performed with the C57B1/6 mouse inbred strain. An inbred strain defines a mouse strain that has been inbred for at least 20 times (Silver, 1995). Mice of an inbred strain are except for their gender genetically identical and virtually homozygous for all genetic loci. This results in a minimal variance among generations and littermates (Beck et al., 2000).

\subsubsection{Mouse inbred strain C57Bl/6}

In 1921, C. C. Little developed the inbred strain C57B1/6 from a mating of Abby Lathrop's stock which also gave rise to C57BR and C57L strains. Strains 6 and 10 separated about 1937.

\subsubsection{SHARP1 and-2 double null-mutant mice}

The generation of SHARP1 mutant mice was performed by replacing exon 3, encoding the DNA-binding domain, by a neomycin resistance cassette (Rossner et al., 2008). Sharp2 knockout mice were created by Sun and colleagues (Sun et al., 2001). Sharp1 and -2 single 
knockout mice were crossed to obtain double mutant mice. All three genotypes were crossed to the $\mathrm{C} 57 \mathrm{Bl} / 6$ background for at least 10 generations.

\subsubsection{Housing conditions of experimental animals}

Mice were kept under a 12/12 hour light/dark (LD) cycle, a constant temperature of about $22^{\circ} \mathrm{C}$ and a humidity of appr. 55\% in standard cages (Makrolon Type2, 19 × 34 x $20 \mathrm{~cm}$ in size) or 'enriched environment' cages (Makrolon Type4, 36 × 50 x $20 \mathrm{~cm}$ in size). 'Enriched environment' cages consisted of two separated compartments: one equipped with a running wheel, stairs, toys and tunnels and the second for drinking and feeding. Food and water were available ad libitum for mice in all housing conditions.

\subsubsection{Experimental animals}

For qRT-PCR, histological, biochemical and behavioural analyses male Sharp1 and -2 double null mutant $\left(\mathrm{S} 1 / 2^{-/-}\right)$mice and WT controls (offspring of S1/2 ${ }^{-/-}$mice crossed to C $57 \mathrm{Bl} / 6$ mice to obtain a WT genotype) aged 4 months were taken.

\subsection{Behavioural tests}

Animals were adapted to experimental rooms and the 12/12 hour LD cycle for at least two weeks prior to analysis. Behavioural tests started at four hours after lights-on (zeitgeber time 4; ZT4). All tests were conducted blind to genotype.

Behavioural analyses were performed according to standard protocols (Crawley, 2000) using equipment by TSE systems, if not indicated otherwise. In between the different trails the equipment was cleaned with $70 \%$ ethanol and water to minimize olfactory cues and to provide identical experimental conditions for all the animals.

\subsubsection{Open field test}

The most commonly test used to analyse spontaneous motor activity is the open field test (Hall, 1934). The open field is a square box $(45 \times 45 \times 55 \mathrm{~cm}$ in size), equipped with red light photobeams that detect movements. The mice were placed in the centre and spontaneous activity was recorded automatically for $10 \mathrm{~min}$ using an infrared tracking system coupled to the computer software VideoMot2 (TSE systems). Total distance, distance travelled in the centre, distance travelled in the periphery, time spent resting, time spent moving, the percentage of time spent in the centre or periphery of the box as well as the mean speed were analysed parameters.

\subsubsection{Hole board test}

The hole board test was used to assess curiosity behaviour (Bradley et al., 1968). Therefore, the open field setup was additionally equipped with a ground plate containing 16 holes with a diameter of $2 \mathrm{~cm}$. Like in the open field test, the mouse was placed in the centre of the box and was allowed to explore it for $10 \mathrm{~min}$. Total distance, number of hole visits (nose pokes) and the amount of time exploring the holes were measured parameters. The mean hole exploration time could be calculated by dividing the accumulated time exploring the holes by the number of nose pokes. 


\subsubsection{Hot plate test}

The hot plate test (O'Callaghan and Holtzman, 1975) provides information about pain sensitivity in animals. Animals were placed onto a $55^{\circ} \mathrm{C}$ hot plate and the latency of showing the first signs of discomfort (e.g. licking the hind limbs or jumping) was measured. A similar sensory processing of WT and mutant mice is a precondition for some learning tests such as fear conditioning.

\subsubsection{Tail suspension test}

To assess motivation or depressive-related behaviour mice were suspended by their tails dangling in the air (Steru et al., 1985). The mice were manually monitored for active and passive behaviour for six minutes. Increased fighting/escaping behaviour resulted in an expanded activity time and was regarded as higher motivation.

\subsection{Sleep deprivation}

Sleep deprivation was performed at the beginning of the light phase for six hours from ZT0ZT6 using the inverted flowerpot technique (Mendelson et al., 1974) which is known to suppress rapid eye movement (REM) sleep and to reduce non rapid eye movement (NREM) sleep. The paradigm was originally described in cats by Jouvet and colleagues (JOUVET et al., 1964) and was later applied to rodents. In this paradigm, each animal is placed in a cage equipped with five platforms ('inverted flowerpots', $2 \mathrm{~cm}$ in diameter) surrounded by water. The animal is able to move between the platforms, but it has to constantly retain muscle tension to avoid falling into the water. When it falls asleep the muscle relaxation accompanied with NREM sleep causes the animal to fall into the water and thus arouses it. Immediately after six hours of SD the animals were sacrified and the brains were rapidly processed using a brain slicer

(see above). 


\section{REFERENCES}

Abe, M., Herzog, E.D., and Block, G.D. (2000). Lithium lengthens the circadian period of individual suprachiasmatic nucleus neurons. Neuroreport 11, 3261-3264.

Aboitiz, F., and Montiel, J. (2003). One hundred million years of interhemispheric communication: the history of the corpus callosum. Braz. J. Med. Biol. Res. 36, 409-420.

Akhtar, R.A., Reddy, A.B., Maywood, E.S., Clayton, J.D., King, V.M., Smith, A.G., Gant, T.W., Hastings, M.H., and Kyriacou, C.P. (2002). Circadian cycling of the mouse liver transcriptome, as revealed by cDNA microarray, is driven by the suprachiasmatic nucleus. Curr. Biol. 12, 540-550.

Alvarez, J.D., and Sehgal, A. (2002). Circadian rhythms: finer clock control. Nature 419, 798-799.

Asato, M.R., Terwilliger, R., Woo, J., and Luna, B. (2010). White Matter Development in Adolescence: A DTI Study. Cerebral Cortex 20, 2122-2131.

Aston, C., Jiang, L., and Sokolov, B.P. (2005). Transcriptional profiling reveals evidence for signaling and oligodendroglial abnormalities in the temporal cortex from patients with major depressive disorder. Mol. Psychiatry 10, 309-322.

Attwell, D., and Laughlin, S.B. (2001). An energy budget for signaling in the grey matter of the brain. J. Cereb. Blood Flow Metab. 21, 1133-1145.

Azmi, S., Sun, H., Ozog, A., and Taneja, R. (2003). mSharp-1/DEC2, a basic helix-loop-helix protein functions as a transcriptional repressor of $\mathrm{E}$ box activity and Stra13 expression. J. Biol. Chem. 278, 20098-20109.

De Bacquer, D., Van Risseghem, M., Clays, E., Kittel, F., De Backer, G., and Braeckman, L. (2009). Rotating shift work and the metabolic syndrome: a prospective study. Int J Epidemiol $38,848-854$.

Bakiri, Y., Burzomato, V., Frugier, G., Hamilton, N.B., Káradóttir, R., and Attwell, D. (2009). Glutamatergic signaling in the brain's white matter. Neuroscience 158, 266-274.

Beck, J.A., Lloyd, S., Hafezparast, M., Lennon-Pierce, M., Eppig, J.T., Festing, M.F., and Fisher, E.M. (2000). Genealogies of mouse inbred strains. Nat. Genet. 24, $23-25$.

Beckman, M. (2004). Neuroscience. Crime, culpability, and the adolescent brain. Science 305, 596-599.

Bengtsson, S.L., Nagy, Z., Skare, S., Forsman, L., Forssberg, H., and Ullen, F. (2005). Extensive piano practicing has regionally specific effects on white matter development. Nat Neurosci $8,1148-1150$.

Birnboim, H.C., and Doly, J. (1979). A rapid alkaline extraction procedure for screening recombinant plasmid DNA. Nucleic Acids Res. 7, 1513-1523.

Bode, B., Shahmoradi, A., Rossner, M.J., and Oster, H. (2011). Genetic interaction of Per1 and Dec1/2 in the regulation of circadian locomotor activity. J. Biol. Rhythms 26, 530-540. 
Bologa, L., Aizenman, Y., Chiappelli, F., and De Vellis, J. (1986). Regulation of myelin basic protein in oligodendrocytes by a soluble neuronal factor. J. Neurosci. Res. 15, 521-528.

Borbély, A.A., and Achermann, P. (1999). Sleep homeostasis and models of sleep regulation. J. Biol. Rhythms 14, 557-568.

Botvinnik, A., Wichert, S.P., Fischer, T.M., and Rossner, M.J. (2010). Integrated analysis of receptor activation and downstream signaling with EXTassays. Nat. Methods 7, 74-80.

Bradley, D.W., Joyce, D., Murphy, E.H., Nash, B.M., Porsolt, R.D., Summerfield, A., and Twyman, W.A. (1968). Amphetamine-barbiturate mixture: effects on the behaviour of mice. Nature 220, 187-188.

Broadhurst, P.L. (1961). Analysis of maternal effects in the inheritance of behaviour. Animal Behaviour 9, 129-141.

Bullitt, E. (1990). Expression of c-fos-like protein as a marker for neuronal activity following noxious stimulation in the rat. J. Comp. Neurol. 296, 517-530.

Bunger, M.K., Wilsbacher, L.D., Moran, S.M., Clendenin, C., Radcliffe, L.A., Hogenesch, J.B., Simon, M.C., Takahashi, J.S., and Bradfield, C.A. (2000). Mop3 is an essential component of the master circadian pacemaker in mammals. Cell 103, 1009-1017.

Bush, G., Luu, P., and Posner, M.I. (2000). Cognitive and emotional influences in anterior cingulate cortex. Trends in Cognitive Sciences 4, 215-222.

Campbell, S.S., Gillin, J.C., Kripke, D.F., Janowsky, D.S., and Risch, S.C. (1989). Lithium delays circadian phase of temperature and REM sleep in a bipolar depressive: a case report. Psychiatry Res 27, 23-29.

Carreiras, M., Seghier, M.L., Baquero, S., Estévez, A., Lozano, A., Devlin, J.T., and Price, C.J. (2009). An anatomical signature for literacy. Nature 461, 983-986.

Carter, C.S., Mintun, M., Nichols, T., and Cohen, J.D. (1997). Anterior cingulate gyrus dysfunction and selective attention deficits in schizophrenia: [15O]H2O PET study during single-trial Stroop task performance. Am J Psychiatry 154, 1670-1675.

Cheo, D.L., Titus, S.A., Byrd, D.R.N., Hartley, J.L., Temple, G.F., and Brasch, M.A. (2004). Concerted Assembly and Cloning of Multiple DNA Segments Using In Vitro Site-Specific Recombination: Functional Analysis of Multi-Segment Expression Clones. Genome Research $14,2111-2120$.

Cho, Y., Noshiro, M., Choi, M., Morita, K., Kawamoto, T., Fujimoto, K., Kato, Y., and Makishima, M. (2009). The Basic Helix-loop-helix Proteins DEC1 and DEC2 Function as Corepressors of Retinoid X Receptors. Mol. Pharmacol.

Cirelli, C. (2009). The genetic and molecular regulation of sleep: from fruit flies to humans. Nat. Rev. Neurosci. 10, 549-560.

Cirelli, Pompeiano, and Tononi (1995). Sleep deprivation and c-fos expression in the rat brain. J Sleep Res 4, 92-106. 
Cirelli, C., Gutierrez, C.M., and Tononi, G. (2004). Extensive and divergent effects of sleep and wakefulness on brain gene expression. Neuron 41, 35-43.

Cirelli, C., Faraguna, U., and Tononi, G. (2006). Changes in brain gene expression after longterm sleep deprivation. J. Neurochem. 98, 1632-1645.

Clinton, S.M., and Meador-Woodruff, J.H. (2004). Abnormalities of the NMDA Receptor and Associated Intracellular Molecules in the Thalamus in Schizophrenia and Bipolar Disorder. Neuropsychopharmacology 29, 1353-1362.

Cohen, H.B., and Dement, W.C. (1965). Sleep: changes in threshold to electroconvulsive shock in rats after deprivation of "paradoxical" phase. Science 150, 1318-1319.

Colgin, L.L. (2011). Oscillations and hippocampal-prefrontal synchrony. Curr. Opin. Neurobiol. 21, 467-474.

Crawley, J.N. (2000). What's wrong with my mouse? Behavioral phenotyping of transgenic and knckout mice (New York: Wiley-Liss).

Dash, M.B., Douglas, C.L., Vyazovskiy, V.V., Cirelli, C., and Tononi, G. (2009). Long-Term Homeostasis of Extracellular Glutamate in the Rat Cerebral Cortex across Sleep and Waking States. J. Neurosci. 29, 620-629.

Demerens, C., Stankoff, B., Logak, M., Anglade, P., Allinquant, B., Couraud, F., Zalc, B., and Lubetzki, C. (1996). Induction of myelination in the central nervous system by electrical activity. Proc Natl Acad Sci U S A 93, 9887-9892.

Demierre, M.-F., Higgins, P.D.R., Gruber, S.B., Hawk, E., and Lippman, S.M. (2005). Statins and cancer prevention. Nat. Rev. Cancer 5, 930-942.

Devan, B.D., Goad, E.H., Petri, H.L., Antoniadis, E.A., Hong, N.S., Ko, C.H., Leblanc, L., Lebovic, S.S., Lo, Q., Ralph, M.R., et al. (2001). Circadian phase-shifted rats show normal acquisition but impaired long-term retention of place information in the water task. Neurobiol Learn Mem 75, 51-62.

Diekelmann, S., and Born, J. (2010). The memory function of sleep. Nature Reviews Neuroscience 11, 114-126.

Van Dongen, H.P.A., Maislin, G., Mullington, J.M., and Dinges, D.F. (2003). The cumulative cost of additional wakefulness: dose-response effects on neurobehavioral functions and sleep physiology from chronic sleep restriction and total sleep deprivation. Sleep 26, 117-126.

Doyle, J.P., Dougherty, J.D., Heiman, M., Schmidt, E.F., Stevens, T.R., Ma, G., Bupp, S., Shrestha, P., Shah, R.D., Doughty, M.L., et al. (2008). Application of a translational profiling approach for the comparative analysis of CNS cell types. Cell 135, 749-762.

Dragunow, M., and Faull, R. (1989). The use of c-fos as a metabolic marker in neuronal pathway tracing. Journal of Neuroscience Methods 29, 261-265.

Du, Y., Fischer, T.Z., Lee, L.N., Lercher, L.D., and Dreyfus, C.F. (2003). Regionally Specific Effects of BDNF on Oligodendrocytes. Developmental Neuroscience 25, 116-126. 
DuBridge, R.B., Tang, P., Hsia, H.C., Leong, P.M., Miller, J.H., and Calos, M.P. (1987). Analysis of mutation in human cells by using an Epstein-Barr virus shuttle system. Mol. Cell. Biol. 7, 379-387.

Dudley, C.A., Erbel-Sieler, C., Estill, S.J., Reick, M., Franken, P., Pitts, S., and McKnight, S.L. (2003). Altered patterns of sleep and behavioral adaptability in NPAS2-deficient mice. Science 301, 379-383.

Dugas, J.C., Tai, Y.C., Speed, T.P., Ngai, J., and Barres, B.A. (2006). Functional genomic analysis of oligodendrocyte differentiation. J. Neurosci. 26, 10967-10983.

Dwork, A.J., Mancevski, B., and Rosoklija, G. (2007). White Matter and Cognitive Function in Schizophrenia. The International Journal of Neuropsychopharmacology 10, 513-536.

Dzirasa, K., McGarity, D.L., Bhattacharya, A., Kumar, S., Takahashi, J.S., Dunson, D., McClung, C.A., and Nicolelis, M.A.L. (2011). Impaired limbic gamma oscillatory synchrony during anxiety-related behavior in a genetic mouse model of bipolar mania. J. Neurosci. 31, 6449-6456.

Eckel-Mahan, K.L., Phan, T., Han, S., Wang, H., Chan, G.C.K., Scheiner, Z.S., and Storm, D.R. (2008). Circadian oscillation of hippocampal MAPK activity and cAmp: implications for memory persistence. Nat. Neurosci. 11, 1074-1082.

Emery, P., and Freeman, M.R. (2007). Glia got rhythm. Neuron 55, 337-339.

Eng, L.F., and Ghirnikar, R.S. (1994). GFAP and astrogliosis. Brain Pathol. 4, 229-237.

Ewer, J., Frisch, B., Hamblen-Coyle, M.J., Rosbash, M., and Hall, J.C. (1992). Expression of the period clock gene within different cell types in the brain of Drosophila adults and mosaic analysis of these cells' influence on circadian behavioral rhythms. J. Neurosci. 12, 33213349.

Eyre, J.A., Miller, S., and Ramesh, V. (1991). Constancy of central conduction delays during development in man: investigation of motor and somatosensory pathways. J. Physiol. (Lond.) $434,441-452$.

Fields, R.D. (2008). White matter in learning, cognition and psychiatric disorders. Trends Neurosci 31, 361-370.

Fields, R.D., and Burnstock, G. (2006). Purinergic signalling in neuron-glia interactions. Nat. Rev. Neurosci. 7, 423-436.

Fields, R.D., and Ni, Y. (2010). Nonsynaptic communication through ATP release from volume-activated anion channels in axons. Sci Signal 3, ra73.

Fields, R.D., and Stevens, B. (2000). ATP: an extracellular signaling molecule between neurons and glia. Trends Neurosci. 23, 625-633.

Flynn, S.W., Lang, D.J., Mackay, A.L., Goghari, V., Vavasour, I.M., Whittall, K.P., Smith, G.N., Arango, V., Mann, J.J., Dwork, A.J., et al. (2003). Abnormalities of myelination in schizophrenia detected in vivo with MRI, and post-mortem with analysis of oligodendrocyte proteins. Mol. Psychiatry $8,811-820$. 
Franken, P., and Dijk, D.-J. (2009). Circadian clock genes and sleep homeostasis. Eur. J. Neurosci. 29, 1820-1829.

Frankland, P.W., and Bontempi, B. (2005). The organization of recent and remote memories. Nat. Rev. Neurosci. 6, 119-130.

Friston, K.J. (1999). Schizophrenia and the disconnection hypothesis. Acta Psychiatr Scand Suppl 395, 68-79.

Fu, L., Pelicano, H., Liu, J., Huang, P., and Lee, C. (2002). The circadian gene Period2 plays an important role in tumor suppression and DNA damage response in vivo. Cell 111, 41-50.

Fujimoto, K., Shen, M., Noshiro, M., Matsubara, K., Shingu, S., Honda, K., Yoshida, E., Suardita, K., Matsuda, Y., and Kato, Y. (2001). Molecular cloning and characterization of DEC2, a new member of basic helix-loop-helix proteins. Biochem. Biophys. Res. Commun. $280,164-171$.

Fusar-Poli, P., Howes, O.D., Allen, P., Broome, M., Valli, I., Asselin, M.-C., Montgomery, A.J., Grasby, P.M., and McGuire, P. (2011). Abnormal prefrontal activation directly related to pre-synaptic striatal dopamine dysfunction in people at clinical high risk for psychosis. Mol. Psychiatry 16, 67-75.

Georgieva, L., Moskvina, V., Peirce, T., Norton, N., Bray, N.J., Jones, L., Holmans, P., Macgregor, S., Zammit, S., Wilkinson, J., et al. (2006). Convergent evidence that oligodendrocyte lineage transcription factor 2 (OLIG2) and interacting genes influence susceptibility to schizophrenia. Proc. Natl. Acad. Sci. U.S.A. 103, 12469-12474.

Gerstner, J.R., Lyons, L.C., Wright, K.P., Loh, D.H., Rawashdeh, O., Eckel-Mahan, K.L., and Roman, G.W. (2009). Cycling behavior and memory formation. J. Neurosci 29, 12824 12830 .

Geyer, M.A. (2008). Developing translational animal models for symptoms of schizophrenia or bipolar mania. Neurotox Res 14, 71-78.

Giedd, J.N. (2004). Structural magnetic resonance imaging of the adolescent brain. Ann. N. Y. Acad. Sci. 1021, 77-85.

Godinho, S.I.H., Maywood, E.S., Shaw, L., Tucci, V., Barnard, A.R., Busino, L., Pagano, M., Kendall, R., Quwailid, M.M., Romero, M.R., et al. (2007). The after-hours mutant reveals a role for Fbx13 in determining mammalian circadian period. Science 316, 897-900.

Goshen, I., Brodsky, M., Prakash, R., Wallace, J., Gradinaru, V., Ramakrishnan, C., and Deisseroth, K. (2011). Dynamics of Retrieval Strategies for Remote Memories. Cell 147, 678-689.

Gottesman, I.I. (2003). The Endophenotype Concept in Psychiatry: Etymology and Strategic Intentions. American Journal of Psychiatry 160, 636-645.

Greene, L.A., and Tischler, A.S. (1976). Establishment of a noradrenergic clonal line of rat adrenal pheochromocytoma cells which respond to nerve growth factor. Proc. Natl. Acad. Sci. U.S.A. 73, 2424-2428. 
Hakak, Y., Walker, J.R., Li, C., Wong, W.H., Davis, K.L., Buxbaum, J.D., Haroutunian, V., and Fienberg, A.A. (2001). Genome-wide expression analysis reveals dysregulation of myelination-related genes in chronic schizophrenia. Proc. Natl. Acad. Sci. U.S.A. 98, 47464751.

Hall, C.S. (1934). Emotional behavior in the rat. I. Defecation and urination as measures of individual differences in emotionality. Journal of Comparative Psychology 18, 385-403.

Hamaguchi, H., Fujimoto, K., Kawamoto, T., Noshiro, M., Maemura, K., Takeda, N., Nagai, R., Furukawa, M., Honma, S., Honma, K., et al. (2004). Expression of the gene for Dec2, a basic helix-loop-helix transcription factor, is regulated by a molecular clock system. Biochem. J. $382,43-50$.

Harrison, P.J., and Weinberger, D.R. (2005). Schizophrenia genes, gene expression, and neuropathology: on the matter of their convergence. Mol. Psychiatry 10, 40-68; image 5.

Hartley, J.L., Temple, G.F., and Brasch, M.A. (2000). DNA cloning using in vitro sitespecific recombination. Genome Res. 10, 1788-1795.

He, Y., Jones, C.R., Fujiki, N., Xu, Y., Guo, B., Holder, J.L., Rossner, M.J., Nishino, S., and Fu, Y.-H. (2009). The transcriptional repressor DEC2 regulates sleep length in mammals. Science 325, 866-870.

Heimer, L. (2000). Basal forebrain in the context of schizophrenia. Brain Res. Brain Res. Rev. $31,205-235$.

Hof, P.R., Haroutunian, V., Friedrich, V.L., Jr, Byne, W., Buitron, C., Perl, D.P., and Davis, K.L. (2003). Loss and altered spatial distribution of oligodendrocytes in the superior frontal gyrus in schizophrenia. Biol. Psychiatry 53, 1075-1085.

Höistad, M., Segal, D., Takahashi, N., Sakurai, T., Buxbaum, J.D., and Hof, P.R. (2009). Linking white and grey matter in schizophrenia: oligodendrocyte and neuron pathology in the prefrontal cortex. Front Neuroanat 3, 9.

Honma, S., Ikeda, M., Abe, H., Tanahashi, Y., Namihira, M., Honma, K., and Nomura, M. (1998). Circadian oscillation of BMAL1, a partner of a mammalian clock gene Clock, in rat suprachiasmatic nucleus. Biochem. Biophys. Res. Commun. 250, 83-87.

Honma, S., Kawamoto, T., Takagi, Y., Fujimoto, K., Sato, F., Noshiro, M., Kato, Y., and Honma, K. (2002). Dec1 and Dec2 are regulators of the mammalian molecular clock. Nature $419,841-844$.

Horne, J. (1988). Why we sleep: The functions of sleep in humans and other mammals (New York, NY, US: Oxford University Press).

Howes, O.D., Montgomery, A.J., Asselin, M.-C., Murray, R.M., Valli, I., Tabraham, P., Bramon-Bosch, E., Valmaggia, L., Johns, L., Broome, M., et al. (2009). Elevated striatal dopamine function linked to prodromal signs of schizophrenia. Arch. Gen. Psychiatry 66, 1320. 
Huang, J.K., Jarjour, A.A., Nait Oumesmar, B., Kerninon, C., Williams, A., Krezel, W., Kagechika, H., Bauer, J., Zhao, C., Evercooren, A.B.-V., et al. (2011). Retinoid X receptor gamma signaling accelerates CNS remyelination. Nat. Neurosci. 14, 45-53.

Hulshoff Pol, H.E., Schnack, H.G., Mandl, R.C.W., Cahn, W., Collins, D.L., Evans, A.C., and Kahn, R.S. (2004). Focal white matter density changes in schizophrenia: reduced interhemispheric connectivity. Neuroimage 21, 27-35.

Impey, S., Obrietan, K., and Storm, D.R. (1999). Making new connections: role of ERK/MAP kinase signaling in neuronal plasticity. Neuron 23, 11-14.

Ishibashi, M., Sasai, Y., Nakanishi, S., and Kageyama, R. (1993). Molecular characterization of HES-2, a mammalian helix-loop-helix factor structurally related to Drosophila hairy and Enhancer of split. Eur. J. Biochem. 215, 645-652.

Ishibashi, T., Dakin, K.A., Stevens, B., Lee, P.R., Kozlov, S.V., Stewart, C.L., and Fields, R.D. (2006). Astrocytes promote myelination in response to electrical impulses. Neuron 49, 823-832.

Ivanova, A.V., Ivanov, S.V., Zhang, X., Ivanov, V.N., Timofeeva, O.A., and Lerman, M.I. (2004). STRA13 interacts with STAT3 and modulates transcription of STAT3-dependent targets. J. Mol. Biol. 340, 641-653.

Jackson, F.R. (2011). Glial cell modulation of circadian rhythms. Glia 59, 1341-1350.

Javanbakht, H., Halwani, R., Cen, S., Saadatmand, J., Musier-Forsyth, K., Gottlinger, H., and Kleiman, L. (2003). The interaction between HIV-1 Gag and human lysyl-tRNA synthetase during viral assembly. J. Biol. Chem. 278, 27644-27651.

Javitt, D.C., and Zukin, S.R. (1991). Recent advances in the phencyclidine model of schizophrenia. Am J Psychiatry 148, 1301-1308.

JOUVET, D., VIMONT, P., DELORME, F., and JOUVET, M. (1964). [STUDY OF SELECTIVE DEPRIVATION OF THE PARADOXAL SLEEP PHASE IN THE CAT]. C. R. Seances Soc. Biol. Fil. 158, 756-759.

Jung, M., Krämer, E., Grzenkowski, M., Tang, K., Blakemore, W., Aguzzi, A., Khazaie, K., Chlichlia, K., Von Blankenfeld, G., and Kettenmann, H. (1995). Lines of murine oligodendroglial precursor cells immortalized by an activated neu tyrosine kinase show distinct degrees of interaction with axons in vitro and in vivo. Eur. J. Neurosci. 7, 1245-1265.

Juraska, J.M., and Kopcik, J.R. (1988). Sex and environmental influences on the size and ultrastructure of the rat corpus callosum. Brain Res. 450, 1-8.

Kanaan, R.A.A., Kim, J.-S., Kaufmann, W.E., Pearlson, G.D., Barker, G.J., and McGuire, P.K. (2005). Diffusion tensor imaging in schizophrenia. Biol. Psychiatry 58, 921-929.

Káradóttir, R., and Attwell, D. (2007). Neurotransmitter receptors in the life and death of oligodendrocytes. Neuroscience 145, 1426-1438. 
Karlsson, B., Knutsson, A., and Lindahl, B. (2001). Is there an association between shift work and having a metabolic syndrome? Results from a population based study of 27,485 people. Occup Environ Med 58, 747-752.

Kibbe, W.A. (2007). OligoCalc: an online oligonucleotide properties calculator. Nucleic Acids Res. 35, W43-46.

Kim, J.S., Kornhuber, H.H., Schmid-Burgk, W., and Holzmüller, B. (1980). Low cerebrospinal fluid glutamate in schizophrenic patients and a new hypothesis on schizophrenia. Neurosci. Lett. 20, 379-382.

Kolodziejczyk, K., Saab, A.S., Nave, K.-A., and Attwell, D. (2010). Why do oligodendrocyte lineage cells express glutamate receptors? F1000 Biol Rep 2, 57.

Kondratov, R.V., Kondratova, A.A., Gorbacheva, V.Y., Vykhovanets, O.V., and Antoch, M.P. (2006). Early aging and age-related pathologies in mice deficient in BMAL1, the core componentof the circadian clock. Genes Dev. 20, 1868-1873.

Kraft, R.H., Mitchell, O.R., Languis, M.L., and Wheatley, G.H. (1980). Hemispheric asymmetries during six- to eight-year-olds performance of Piagetian conservation and reading tasks. Neuropsychologia 18, 637-643.

Kubicki, M., McCarley, R., Westin, C.-F., Park, H.-J., Maier, S., Kikinis, R., Jolesz, F.A., and Shenton, M.E. (2007). A review of diffusion tensor imaging studies in schizophrenia. J Psychiatr Res 41, 15-30.

Kukley, M., Capetillo-Zarate, E., and Dietrich, D. (2007). Vesicular glutamate release from axons in white matter. Nat. Neurosci. 10, 311-320.

Lajtha, A., Toth, J., Fujimoto, K., and Agrawal, H.C. (1977). Turnover of myelin proteins in mouse brain in vivo. Biochem. J. 164, 323-329.

Lee, P.R., and Fields, R.D. (2009). Regulation of myelin genes implicated in psychiatric disorders by functional activity in axons. Front Neuroanat 3, 4 .

Lenox, R.H., Gould, T.D., and Manji, H.K. (2002). Endophenotypes in bipolar disorder. Am. J. Med. Genet. 114, 391-406.

Li, Y., Song, X., Ma, Y., Liu, J., Yang, D., and Yan, B. (2004). DNA binding, but not interaction with Bmal1, is responsible for DEC1-mediated transcription regulation of the circadian gene mPer1. Biochem. J. 382, 895-904.

Li, Y., Xie, M., Yang, J., Yang, D., Deng, R., Wan, Y., and Yan, B. (2006). The expression of antiapoptotic protein survivin is transcriptionally upregulated by DEC1 primarily through multiple sp1 binding sites in the proximal promoter. Oncogene 25, 3296-3306.

Lim, C., Chung, B.Y., Pitman, J.L., McGill, J.J., Pradhan, S., Lee, J., Keegan, K.P., Choe, J., and Allada, R. (2007). Clockwork orange encodes a transcriptional repressor important for circadian-clock amplitude in Drosophila. Curr. Biol. 17, 1082-1089. 
Lin, S.-C., Huck, J.H.J., Roberts, J.D.B., Macklin, W.B., Somogyi, P., and Bergles, D.E. (2005). Climbing fiber innervation of NG2-expressing glia in the mammalian cerebellum. Neuron 46, 773-785.

Liston, C., Watts, R., Tottenham, N., Davidson, M.C., Niogi, S., Ulug, A.M., and Casey, B.J. (2006). Frontostriatal microstructure modulates efficient recruitment of cognitive control. Cereb. Cortex 16, 553-560.

LOWRY, O.H., ROSEBROUGH, N.J., FARR, A.L., and RANDALL, R.J. (1951). Protein measurement with the Folin phenol reagent. J. Biol. Chem. 193, 265-275.

Mabbott, D.J., Noseworthy, M., Bouffet, E., Laughlin, S., and Rockel, C. (2006). White matter growth as a mechanism of cognitive development in children. Neuroimage 33, 936946.

Mackiewicz, M., Shockley, K.R., Romer, M.A., Galante, R.J., Zimmerman, J.E., Naidoo, N., Baldwin, D.A., Jensen, S.T., Churchill, G.A., and Pack, A.I. (2007). Macromolecule biosynthesis: a key function of sleep. Physiol. Genomics 31, 441-457.

Madsen, P.L., Schmidt, J.F., Wildschiødtz, G., Friberg, L., Holm, S., Vorstrup, S., and Lassen, N.A. (1991). Cerebral O2 metabolism and cerebral blood flow in humans during deep and rapid-eye-movement sleep. J. Appl. Physiol. 70, 2597-2601.

Mansour, H.A., Monk, T.H., and Nimgaonkar, V.L. (2005). Circadian genes and bipolar disorder. Ann. Med. 37, 196-205.

Mansour, H.A., Talkowski, M.E., Wood, J., Chowdari, K.V., McClain, L., Prasad, K., Montrose, D., Fagiolini, A., Friedman, E.S., Allen, M.H., et al. (2009). Association study of 21 circadian genes with bipolar I disorder, schizoaffective disorder, and schizophrenia. Bipolar Disord 11, 701-710.

Maquet, P. (1995). Sleep function(s) and cerebral metabolism. Behav. Brain Res. 69, 75-83.

Markham, J.A., and Greenough, W.T. (2004). Experience-driven brain plasticity: beyond the synapse. Neuron Glia Biol. 1, 351-363.

Markham, J.A., Herting, M.M., Luszpak, A.E., Juraska, J.M., and Greenough, W.T. (2009). Myelination of the corpus callosum in male and female rats following complex environment housing during adulthood. Brain Res. 1288, 9-17.

Mason, J.L., and Goldman, J.E. (2002). A2B5+ and O4+ Cycling progenitors in the adult forebrain white matter respond differentially to PDGF-AA, FGF-2, and IGF-1. Mol. Cell. Neurosci. 20, 30-42.

Masri, S., and Sassone-Corsi, P. (2010). Plasticity and specificity of the circadian epigenome. Nat Neurosci 13, 1324-1329.

Masubuchi, S., Honma, S., Abe, H., Ishizaki, K., Namihira, M., Ikeda, M., and Honma, K. (2000). Clock genes outside the suprachiasmatic nucleus involved in manifestation of locomotor activity rhythm in rats. Eur. J. Neurosci. 12, 4206-4214. 
Mendelson, W.B., Guthrie, R.D., Frederick, G., and Jed Wyatt, R. (1974). The flower pot technique of Rapid Eye Movement (REM) sleep deprivation. Pharmacology Biochemistry and Behavior 2, 553-556.

Michailov, G.V., Sereda, M.W., Brinkmann, B.G., Fischer, T.M., Haug, B., Birchmeier, C., Role, L., Lai, C., Schwab, M.H., and Nave, K.-A. (2004). Axonal neuregulin-1 regulates myelin sheath thickness. Science 304, 700-703.

Ming, T. (2000). Schizophrenia: genes and environment. Biological Psychiatry 47, 210-220.

Mukherjee, S., Coque, L., Cao, J.-L., Kumar, J., Chakravarty, S., Asaithamby, A., Graham, A., Gordon, E., Enwright, J.F., 3rd, DiLeone, R.J., et al. (2010). Knockdown of Clock in the ventral tegmental area through RNA interference results in a mixed state of mania and depression-like behavior. Biol. Psychiatry 68, 503-511.

Mullis, K., Faloona, F., Scharf, S., Saiki, R., Horn, G., and Erlich, H. (1986). Specific enzymatic amplification of DNA in vitro: the polymerase chain reaction. Cold Spring Harb. Symp. Quant. Biol. 51 Pt 1, 263-273.

Murre, C., Bain, G., Van Dijk, M.A., Engel, I., Furnari, B.A., Massari, M.E., Matthews, J.R., Quong, M.W., Rivera, R.R., and Stuiver, M.H. (1994). Structure and function of helix-loophelix proteins. Biochimica Et Biophysica Acta (BBA) - Gene Structure and Expression 1218, 129-135.

Nagy, Z., Westerberg, H., and Klingberg, T. (2004). Maturation of white matter is associated with the development of cognitive functions during childhood. J Cogn Neurosci 16, 12271233.

Nakajima, Y., Ikeda, M., Kimura, T., Honma, S., Ohmiya, Y., and Honma, K. (2004). Bidirectional role of orphan nuclear receptor RORalpha in clock gene transcriptions demonstrated by a novel reporter assay system. FEBS Lett. 565, 122-126.

Nakashima, A., Kawamoto, T., Honda, K.K., Ueshima, T., Noshiro, M., Iwata, T., Fujimoto, K., Kubo, H., Honma, S., Yorioka, N., et al. (2008). DEC1 modulates the circadian phase of clock gene expression. Mol. Cell. Biol. 28, 4080-4092.

Nave, K.-A. (2010). Myelination and the trophic support of long axons. Nat. Rev. Neurosci. $11,275-283$.

Nave, K.-A., and Salzer, J.L. (2006). Axonal regulation of myelination by neuregulin 1. Curr. Opin. Neurobiol. 16, 492-500.

Nestler, E.J., and Hyman, S.E. (2010). Animal models of neuropsychiatric disorders. Nat. Neurosci. 13, 1161-1169.

Nieuwenhuis, S., Ridderinkhof, K.R., Blom, J., Band, G.P.H., and Kok, A. (2001). Error-related brain potentials are differentially related to awareness of response errors: Evidence from an antisaccade task. Psychophysiology 38, 752-760.

Nithianantharajah, J., and Hannan, A.J. (2006). Enriched environments, experience-dependent plasticity and disorders of the nervous system. Nat. Rev. Neurosci. 7, 697-709. 
O'Callaghan, J.P., and Holtzman, S.G. (1975). Quantification of the analgesic activity of narcotic antagonists by a modified hot-plate procedure. J. Pharmacol. Exp. Ther. 192, 497505 .

Oishi, K., Fukui, H., and Ishida, N. (2000). Rhythmic expression of BMAL1 mRNA is altered in Clock mutant mice: differential regulation in the suprachiasmatic nucleus and peripheral tissues. Biochem. Biophys. Res. Commun. 268, 164-171.

Panda, S., Antoch, M.P., Miller, B.H., Su, A.I., Schook, A.B., Straume, M., Schultz, P.G., Kay, S.A., Takahashi, J.S., and Hogenesch, J.B. (2002). Coordinated transcription of key pathways in the mouse by the circadian clock. Cell 109, 307-320.

Pando, M.P., Morse, D., Cermakian, N., and Sassone-Corsi, P. (2002). Phenotypic rescue of a peripheral clock genetic defect via SCN hierarchical dominance. Cell 110, 107-117.

Power, J., Mayer-Pröschel, M., Smith, J., and Noble, M. (2002). Oligodendrocyte Precursor Cells from Different Brain Regions Express Divergent Properties Consistent with the Differing Time Courses of Myelination in These Regions. Developmental Biology 245, 362375.

Preitner, N., Damiola, F., Lopez-Molina, L., Zakany, J., Duboule, D., Albrecht, U., and Schibler, U. (2002). The orphan nuclear receptor REV-ERBalpha controls circadian transcription within the positive limb of the mammalian circadian oscillator. Cell 110, 251260.

Prolo, L.M., Takahashi, J.S., and Herzog, E.D. (2005). Circadian rhythm generation and entrainment in astrocytes. J. Neurosci. 25, 404-408.

Quallo, M.M., Price, C.J., Ueno, K., Asamizuya, T., Cheng, K., Lemon, R.N., and Iriki, A. (2009). Gray and white matter changes associated with tool-use learning in macaque monkeys. Proc. Natl. Acad. Sci. U.S.A. 106, 18379-18384.

Rechtschaffen, A. (1998). Current perspectives on the function of sleep. Perspect. Biol. Med. 41, 359-390.

Reick, M., Garcia, J.A., Dudley, C., and McKnight, S.L. (2001). NPAS2: an analog of clock operative in the mammalian forebrain. Science 293, 506-509.

Reppert, S.M., and Weaver, D.R. (2001). Molecular analysis of mammalian circadian rhythms. Annu. Rev. Physiol. 63, 647-676.

Reppert, S.M., and Weaver, D.R. (2002). Coordination of circadian timing in mammals. Nature 418, 935-941.

Richier, B., Michard-Vanhée, C., Lamouroux, A., Papin, C., and Rouyer, F. (2008). The clockwork orange Drosophila protein functions as both an activator and a repressor of clock gene expression. J. Biol. Rhythms 23, 103-116.

Riederer, B.M., Berbel, P., and Innocenti, G.M. (2004). Neurons in the corpus callosum of the cat during postnatal development. Eur. J. Neurosci. 19, 2039-2046. 
Del Rio-Hortega, P. (1928). Tercera aportacion al conocimiento morfologico e interpretacion functional de la oligodendroglia. Mem Real Soc Esp Hist Nat 40-122.

Ross, S.E., Greenberg, M.E., and Stiles, C.D. (2003). Basic helix-loop-helix factors in cortical development. Neuron 39, 13-25.

Rossner, M.J., Dörr, J., Gass, P., Schwab, M.H., and Nave, K.A. (1997). SHARPs: mammalian enhancer-of-split- and hairy-related proteins coupled to neuronal stimulation. Mol. Cell. Neurosci. 9, 460-475.

Rossner, M.J., Oster, H., Wichert, S.P., Reinecke, L., Wehr, M.C., Reinecke, J., Eichele, G., Taneja, R., and Nave, K.-A. (2008). Disturbed clockwork resetting in Sharp-1 and Sharp-2 single and double mutant mice. PLoS ONE 3, e2762.

Roy, K., Murtie, J.C., El-Khodor, B.F., Edgar, N., Sardi, S.P., Hooks, B.M., Benoit-Marand, M., Chen, C., Moore, H., O'Donnell, P., et al. (2007). Loss of erbB signaling in oligodendrocytes alters myelin and dopaminergic function, a potential mechanism for neuropsychiatric disorders. Proceedings of the National Academy of Sciences 104, 81318136.

Roybal, K., Theobold, D., Graham, A., DiNieri, J.A., Russo, S.J., Krishnan, V., Chakravarty, S., Peevey, J., Oehrlein, N., Birnbaum, S., et al. (2007). Mania-like behavior induced by disruption of CLOCK. Proc. Natl. Acad. Sci. U.S.A. 104, 6406-6411.

Rudic, R.D., McNamara, P., Curtis, A.-M., Boston, R.C., Panda, S., Hogenesch, J.B., and Fitzgerald, G.A. (2004). BMAL1 and CLOCK, two essential components of the circadian clock, are involved in glucose homeostasis. PLoS Biol. 2, e377.

Rutter, J., Reick, M., and McKnight, S.L. (2002). Metabolism and the control of circadian rhythms. Annu. Rev. Biochem. 71, 307-331.

Safferman, A.Z., Kane, J.M., Aronowitz, J.S., Gordon, M.F., Pollack, S., and Lieberman, J.A. (1994). The use of clozapine in neurologic disorders. J Clin Psychiatry 55 Suppl B, 98-101.

Salvatore, P., Ghidini, S., Zita, G., De Panfilis, C., Lambertino, S., Maggini, C., and Baldessarini, R.J. (2008). Circadian activity rhythm abnormalities in ill and recovered bipolar I disorder patients. Bipolar Disord 10, 256-265.

Sambrook, J., and Russell, D. (2001). Molecular cloning. A laboratory manual (New York: Cold Spring Harbor Press).

Sanger, F., Nicklen, S., and Coulson, A.R. (1977). DNA sequencing with chain-terminating inhibitors. Proc. Natl. Acad. Sci. U.S.A. 74, 5463-5467.

Sato, F., Kawamoto, T., Fujimoto, K., Noshiro, M., Honda, K.K., Honma, S., Honma, K., and Kato, Y. (2004a). Functional analysis of the basic helix-loop-helix transcription factor DEC1 in circadian regulation. Interaction with BMAL1. Eur. J. Biochem. 271, 4409-4419.

Sato, T.K., Panda, S., Miraglia, L.J., Reyes, T.M., Rudic, R.D., McNamara, P., Naik, K.A., FitzGerald, G.A., Kay, S.A., and Hogenesch, J.B. (2004b). A functional genomics strategy reveals Rora as a component of the mammalian circadian clock. Neuron 43, 527-537. 
Scharf, M.T., Naidoo, N., Zimmerman, J.E., and Pack, A.I. (2008). The energy hypothesis of sleep revisited. Prog. Neurobiol. 86, 264-280.

Scholz, J., Klein, M.C., Behrens, T.E.J., and Johansen-Berg, H. (2009). Training induces changes in white-matter architecture. Nat Neurosci 12, 1370-1371.

Segal, D., Schmitz, C., and Hof, P.R. (2009). Spatial distribution and density of oligodendrocytes in the cingulum bundle are unaltered in schizophrenia. Acta Neuropathol. $117,385-394$.

Sehgal, A. (2004). Molecular biology of circadian rhythms (Wiley-IEEE).

Shen, M., Kawamoto, T., Yan, W., Nakamasu, K., Tamagami, M., Koyano, Y., Noshiro, M., and Kato, Y. (1997). Molecular Characterization of the Novel Basic Helix-Loop-Helix Protein DEC1 Expressed in Differentiated Human Embryo Chondrocytes. Biochemical and Biophysical Research Communications 236, 294-298.

Silver, L.M. (1995). Mouse Genetics: Concepts and Applications (Oxford University Press, USA).

Singh, H., and Jungalwala, F.B. (1979). The turnover of myelin proteins in adult rat brain. Int. J. Neurosci. 9, 123-131.

Sirevaag, A.M., and Greenough, W.T. (1987). Differential rearing effects on rat visual cortex synapses. III. Neuronal and glial nuclei, boutons, dendrites, and capillaries. Brain Res. 424, $320-332$.

Siwicki, K.K., Eastman, C., Petersen, G., Rosbash, M., and Hall, J.C. (1988). Antibodies to the period gene product of Drosophila reveal diverse tissue distribution and rhythmic changes in the visual system. Neuron 1, 141-150.

Smeraldi, E., Benedetti, F., Barbini, B., Campori, E., and Colombo, C. (1999). Sustained antidepressant effect of sleep deprivation combined with pindolol in bipolar depression. A placebo-controlled trial. Neuropsychopharmacology 20,380-385.

Spanagel, R., Pendyala, G., Abarca, C., Zghoul, T., Sanchis-Segura, C., Magnone, M.C., Lascorz, J., Depner, M., Holzberg, D., Soyka, M., et al. (2005). The clock gene Per2 influences the glutamatergic system and modulates alcohol consumption. Nat. Med. 11, 3542.

Stark, A.K., Uylings, H.B.M., Sanz-Arigita, E., and Pakkenberg, B. (2004). Glial cell loss in the anterior cingulate cortex, a subregion of the prefrontal cortex, in subjects with schizophrenia. Am J Psychiatry 161, 882-888.

Stephan, F.K., and Kovacevic, N.S. (1978). Multiple retention deficit in passive avoidance in rats is eliminated by suprachiasmatic lesions. Behav Biol 22, 456-462.

Steriade, M., and Hobson, J. (1976). Neuronal activity during the sleep-waking cycle. Prog. Neurobiol. 6, 155-376.

Steru, L., Chermat, R., Thierry, B., and Simon, P. (1985). The tail suspension test: a new method for screening antidepressants in mice. Psychopharmacology (Berl.) 85, 367-370. 
Stevens, B., and Fields, R.D. (2000). Response of Schwann cells to action potentials in development. Science 287, 2267-2271.

Stevens, B., Porta, S., Haak, L.L., Gallo, V., and Fields, R.D. (2002). Adenosine: a neuronglial transmitter promoting myelination in the CNS in response to action potentials. Neuron $36,855-868$.

Stewart, S.E., Platko, J., Fagerness, J., Birns, J., Jenike, E., Smoller, J.W., Perlis, R., Leboyer, M., Delorme, R., Chabane, N., et al. (2007). A genetic family-based association study of OLIG2 in obsessive-compulsive disorder. Arch. Gen. Psychiatry 64, 209-214.

Storch, K.-F., Lipan, O., Leykin, I., Viswanathan, N., Davis, F.C., Wong, W.H., and Weitz, C.J. (2002). Extensive and divergent circadian gene expression in liver and heart. Nature 417, $78-83$.

Suh, J., and Jackson, F.R. (2007). Drosophila ebony activity is required in glia for the circadian regulation of locomotor activity. Neuron 55, 435-447.

Sun, H., Lu, B., Li, R.Q., Flavell, R.A., and Taneja, R. (2001). Defective T cell activation and autoimmune disorder in Stra13-deficient mice. Nat. Immunol. 2, 1040-1047.

Swadlow, H.A. (1985). Physiological properties of individual cerebral axons studied in vivo for as long as one year. J. Neurophysiol. 54, 1346-1362.

Sweatt, J.D. (2004). Mitogen-activated protein kinases in synaptic plasticity and memory. Curr. Opin. Neurobiol. 14, 311-317.

Szeligo, F., and Leblond, C.P. (1977). Response of the three main types of glial cells of cortex and corpus callosum in rats handled during suckling or exposed to enriched, control and impoverished environments following weaning. J. Comp. Neurol. 172, 247-263.

Takahashi, N., Sakurai, T., Davis, K.L., and Buxbaum, J.D. (2011). Linking oligodendrocyte and myelin dysfunction to neurocircuitry abnormalities in schizophrenia. Prog. Neurobiol. 93, $13-24$.

Takeda, M., Nelson, D.J., and Soliven, B. (1995). Calcium signaling in cultured rat oligodendrocytes. Glia 14, 225-236.

Tanaka, H., Ma, J., Tanaka, K.F., Takao, K., Komada, M., Tanda, K., Suzuki, A., Ishibashi, T., Baba, H., Isa, T., et al. (2009). Mice with altered myelin proteolipid protein gene expression display cognitive deficits accompanied by abnormal neuron-glia interactions and decreased conduction velocities. J. Neurosci. 29, 8363-8371.

Tapp, W.N., and Holloway, F.A. (1981). Phase shifting circadian rhythms produces retrograde amnesia. Science 211, 1056-1058.

Teboul, M., Guillaumond, F., Gréchez-Cassiau, A., and Delaunay, F. (2008). The nuclear hormone receptor family round the clock. Mol. Endocrinol. 22, 2573-2582.

Terao, A., Wisor, J.P., Peyron, C., Apte-Deshpande, A., Wurts, S.W., Edgar, D.M., and Kilduff, T.S. (2006). Gene expression in the rat brain during sleep deprivation and recovery sleep: an Affymetrix GeneChip study. Neuroscience 137, 593-605. 
Terman, J.S., Terman, M., Lo, E.-S., and Cooper, T.B. (2001). Circadian Time of Morning Light Administration and Therapeutic Response in Winter Depression. Arch Gen Psychiatry $58,69-75$.

Thaker, G.K. (2008). Neurophysiological endophenotypes across bipolar and schizophrenia psychosis. Schizophr Bull 34, 760-773.

Tkachev, D., Mimmack, M.L., Ryan, M.M., Wayland, M., Freeman, T., Jones, P.B., Starkey, M., Webster, M.J., Yolken, R.H., and Bahn, S. (2003). Oligodendrocyte dysfunction in schizophrenia and bipolar disorder. Lancet 362, 798-805.

Tkachev, D., Mimmack, M.L., Huffaker, S.J., Ryan, M., and Bahn, S. (2007). Further evidence for altered myelin biosynthesis and glutamatergic dysfunction in schizophrenia. Int. J. Neuropsychopharmacol. 10, 557-563.

Towbin, H., Staehelin, T., and Gordon, J. (1979). Electrophoretic transfer of proteins from polyacrylamide gels to nitrocellulose sheets: procedure and some applications. Proc. Natl. Acad. Sci. U.S.A. 76, 4350-4354.

Tsai, G., and Coyle, J.T. (1995). N-acetylaspartate in neuropsychiatric disorders. Prog. Neurobiol. 46, 531-540.

Tugores, A., Rubio, T., Rancaño, C., and Alonso, M.A. (1997). A tandem array of Sp-1 sites and a reverse initiator element are both required for synergistic transcriptional activation of the T-cell-specific MAL gene. DNA Cell Biol. 16, 245-255.

Turek, F.W., Joshu, C., Kohsaka, A., Lin, E., Ivanova, G., McDearmon, E., Laposky, A., Losee-Olson, S., Easton, A., Jensen, D.R., et al. (2005). Obesity and metabolic syndrome in circadian Clock mutant mice. Science 308, 1043-1045.

Ueda, H.R., Matsumoto, A., Kawamura, M., Iino, M., Tanimura, T., and Hashimoto, S. (2002). Genome-wide transcriptional orchestration of circadian rhythms in Drosophila. J. Biol. Chem. 277, 14048-14052.

Ueda, H.R., Hayashi, S., Chen, W., Sano, M., Machida, M., Shigeyoshi, Y., Iino, M., and Hashimoto, S. (2005). System-level identification of transcriptional circuits underlying mammalian circadian clocks. Nat. Genet. 37, 187-192.

Uranova, N., Orlovskaya, D., Vikhreva, O., Zimina, I., Kolomeets, N., Vostrikov, V., and Rachmanova, V. (2001). Electron microscopy of oligodendroglia in severe mental illness. Brain Res. Bull. 55, 597-610.

Uranova, N.A., Vostrikov, V.M., Orlovskaya, D.D., and Rachmanova, V.I. (2004). Oligodendroglial density in the prefrontal cortex in schizophrenia and mood disorders: a study from the Stanley Neuropathology Consortium. Schizophr. Res. 67, 269-275.

Wake, H., Lee, P.R., and Fields, R.D. (2011). Control of local protein synthesis and initial events in myelination by action potentials. Science 333, 1647-1651.

Wang, H., and Storm, D.R. (2003). Calmodulin-regulated adenylyl cyclases: cross-talk and plasticity in the central nervous system. Mol. Pharmacol. 63, 463-468. 
Weissman, D.H., Gopalakrishnan, A., Hazlett, C.J., and Woldorff, M.G. (2005). Dorsal Anterior Cingulate Cortex Resolves Conflict from Distracting Stimuli by Boosting Attention Toward Relevant Events. Cereb. Cortex 15, 229-237.

Wisor, J.P., O’Hara, B.F., Terao, A., Selby, C.P., Kilduff, T.S., Sancar, A., Edgar, D.M., and Franken, P. (2002). A role for cryptochromes in sleep regulation. BMC Neurosci 3, 20.

Witthaus, H., Brüne, M., Kaufmann, C., Bohner, G., Ozgürdal, S., Gudlowski, Y., Heinz, A., Klingebiel, R., and Juckel, G. (2008). White matter abnormalities in subjects at ultra high-risk for schizophrenia and first-episode schizophrenic patients. Schizophr. Res. 102, 141-149.

Wright, I.C., Rabe-Hesketh, S., Woodruff, P.W., David, A.S., Murray, R.M., and Bullmore, E.T. (2000). Meta-analysis of regional brain volumes in schizophrenia. Am J Psychiatry 157, $16-25$.

Wulff, K., Gatti, S., Wettstein, J.G., and Foster, R.G. (2010). Sleep and circadian rhythm disruption in psychiatric and neurodegenerative disease. Nat Rev Neurosci 11, 589-599.

Xu, H., Yang, H.-J., Zhang, Y., Clough, R., Browning, R., and Li, X.-M. (2009). Behavioral and neurobiological changes in C57BL/6 mice exposed to cuprizone. Behav. Neurosci. 123, 418-429.

Yakovlev, P.I., and Lecours, A.R. (1967). The myelogenetic cycles of regional maturation of the brain. 3-70.

Yeh, T.-H., Lee, D.Y., Gianino, S.M., and Gutmann, D.H. (2009). Microarray analyses reveal regional astrocyte heterogeneity with implications for neurofibromatosis type 1 (NF1)regulated glial proliferation. Glia 57, 1239-1249.

Yu, W., Nomura, M., and Ikeda, M. (2002). Interactivating feedback loops within the mammalian clock: BMAL1 is negatively autoregulated and upregulated by CRY1, CRY2, and PER2. Biochem. Biophys. Res. Commun. 290, 933-941.

Yun, Z., Maecker, H.L., Johnson, R.S., and Giaccia, A.J. (2002). Inhibition of PPAR gamma 2 gene expression by the HIF-1-regulated gene DEC1/Stra13: a mechanism for regulation of adipogenesis by hypoxia. Dev. Cell 2, 331-341.

Zalc, B., and Fields, R.D. (2000). Do Action Potentials Regulate Myelination? Neuroscientist $6,5-13$.

Zhang, Y., and Barres, B.A. (2010). Astrocyte heterogeneity: an underappreciated topic in neurobiology. Current Opinion in Neurobiology 20, 588-594. 


\section{AbBreviations}

A

ACC

ApoA1

Appr.

ATCC

AmpR

ATP

b

bp

BMAL1

${ }^{\circ} \mathrm{C}$

$\mathrm{CC}$

$\mathrm{CCO}$

cDNA

CMV

CNP

CNS

CRY

CSF

CT

Cyc1

Cx

D

DMEM

DMSO

DNA

DNase

dNTP

ds

DTT

E.coli

EDTA

EEG

e.g.

et al.

$\mathrm{EtOH}$

EYFP

EXT

F

FBS

f.c.

Fig.

fwd

$\mathrm{g}$

GFAP

$\mathrm{h}$
Ampere, Absorbance

Anterior cingulated cortex

Apolipoprotein A-1

Approximately

American Tissue Culture Collection

Ampicillin resistance

Adenosine triphosphate

Basic

Base pairs

Brain and Muscle ARNT-Like Protein 1

Degree Celsius

Corpus callosum

core clock oscillator

Complementary DNA

Cytomegalovirus

2',3'-Cyclic nucleotide 3'-phosphodiesterase

Central nervous system

Cryptochrome

Cerebrospinal fliud

circadian time

Cytochrome C-1

Cortex

Dalton

Dulbeccos's modified Eagle Medium

Dimethyl sulfoxid

Deoxyribonucleic acid

Deoxyribonuclease

Deoxyribonucleotide triphosphate

double stranded

1,4-Dithiothreitol

Escherichia coli

Ethylendiamintetraacetate

Electroencephalogram

For example (from Latin exempli gratia)

And others

Ethanol

Enhanced Yellow Fluorescent Protein

Expressed unique sequence tag

Farad

Fetal bovine serum

Final concentration

Figure

Forward

Gram, gravity

Glial fibrillary acidic protein

Hour 


\begin{tabular}{|c|c|}
\hline HLH & Helix loop helix \\
\hline HMGCR & 3-hydroxy-3-methylglutaryl-CoA reductase \\
\hline HS & Horse Serum \\
\hline HRP & Horse radish peroxidase \\
\hline $\mathrm{Hz}$ & Hertz (s-1) \\
\hline IEG & immediate-early gene \\
\hline KanR & Kanamycin resistance \\
\hline $\mathrm{L}$ & Liter \\
\hline LB & Luria and Bertani medium (or Luria's broth) \\
\hline $\mathrm{LCM}$ & Laser-captured microdissection \\
\hline Luc & Luciferase \\
\hline $\mathrm{m}$ & Mouse, milli (10-3) \\
\hline $\mathrm{M}$ & Molar \\
\hline$\mu$ & Micro (10-6) \\
\hline MAL & Myelin and lymphocyte protein \\
\hline MBP & Myelin basic protein \\
\hline $\min$ & Minute \\
\hline mRNA & messenger RNA \\
\hline $\mathrm{n}$ & Number, nano (10-9) \\
\hline ns & Not significant \\
\hline NREM & Non rapid eye movement \\
\hline OD & Optical density \\
\hline OLIG2 & Oligodendrocyte lineage transcription factor 2 \\
\hline $\mathrm{OPC}$ & Oligodendrocyte precursor cell \\
\hline ORF & Open reading frame \\
\hline $\mathrm{p}$ & Plasmid, pico (10-9) \\
\hline PAS & PER-ARNT-SIM \\
\hline PBS & Phosphate-buffered saline \\
\hline PCR & Polymerase chain reaction \\
\hline PEG & Polyethylene glycole \\
\hline PER & Period \\
\hline $\mathrm{PFC}$ & Prefrontal cortex \\
\hline $\mathrm{pH}$ & Negative decimal logarithm of the hydrogen ions $(\mathrm{H}+)$ \\
\hline PLL & Poly-L-lysin \\
\hline PLP & Proteolipid protein \\
\hline PNS & Peripheral nervous system \\
\hline Prom & Promoter \\
\hline $\mathrm{R}$ & Resistance \\
\hline $\mathrm{rb}$ & Rabbit \\
\hline REM & Rapid eye movement \\
\hline rev & Reverse \\
\hline RGC & Retinal ganglion cell \\
\hline RHT & Retinohypothalamic tract \\
\hline RLU & Relative luciferase unit \\
\hline RNA & Ribonucleic acid \\
\hline RNase & Ribonuclease \\
\hline rpm & Rotations per minute \\
\hline RT & Room temperature \\
\hline S & Second \\
\hline
\end{tabular}




$\begin{array}{ll}\text { S1/2-/- } & \text { SHARP1/2-/- } \\ \text { SCN } & \text { Suprachiasmatic nucleus } \\ \text { SEM } & \text { Standard error of the mean } \\ \text { SD } & \text { Sleep deprivation, standard deviation } \\ \text { SDS } & \text { Sodium dodecyl sulfate } \\ \text { SHARP } & \text { Enhancer of split and hairy-related protein } \\ \text { SOC } & \text { Super optimal broth medium with catabolite repression } \\ \text { Ss } & \text { Single stranded } \\ \text { SSMCx } & \text { Somatosensory motor cortex } \\ \text { SQS } & \text { Squalene synthase } \\ \text { SV40 } & \text { Simian virus 40 } \\ \text { SWA } & \text { Slow wave activity } \\ \tau & \text { tau, circadian period length } \\ \text { Tab. } & \text { Table } \\ \text { Taq } & \text { Thermus aquaticus } \\ \text { TK } & \text { Thymidinkinase promotor (from herpes simplex virus) } \\ \text { Tm } & \text { Melting temperature } \\ \text { Tris } & \text { Tris(hydroxymethyl)aminomethane } \\ \text { tRNA } & \text { transfer RNA } \\ \text { U } & \text { Unit } \\ \text { UV } & \text { Ultraviolet } \\ \text { v/v } & \text { Volume/volume } \\ \text { V } & \text { Volt } \\ \text { W } & \text { Watt } \\ \text { w/v } & \text { Weight/volume } \\ \text { WT } & \text { Wild type } \\ \text { ZT } & \text { Zeitgeber time } \\ & \end{array}$




\title{
10 Curriculum Vitae
}

\section{Personal data}

Name

Date of birth

Place of birth

Nationality

\author{
Lisa Reinecke, maiden name Bitterberg \\ 16.04.1981 \\ Höxter \\ German
}

\section{Education}

2007-current

\section{Graduate student}

Prof. Nave, Department of Neurogenetics

Max-Planck-Institute of Experimental Medicine, Göttingen

2006-2007

\section{Diploma student}

Prof. Nave, Department of Neurogenetics

Max-Planck-Institute of Experimental Medicine, Göttingen

2006

Diploma in biology

Speciality in Biochemistry

Georg-August-University, Göttingen

2000

Abitur

König-Wilhelm-Gymnasium, Höxter

$1991-2000$

\section{Gymnasium}

König-Wilhelm-Gymnasium, Höxter

1987-1991

Grundschule / Primary school

Heinrich-Sohnrey-Grundschule, Boffzen

Professional Qualifications

2001-2006

\section{Research assistant}

Prof. Nave, Department of Neurogenetics

Max-Planck-Institute of Experimental Medicine, Göttingen

\section{Internship}

Prof. Jahn, Department of Neurobiology

Max-Planck-Institute of Biophysical Chemistry, Göttingen

\section{Internship}

Prof. Nave, Department of Neurogenetics

Max-Planck-Institute of Experimental Medicine, Göttingen 


\section{Publications}

Reinecke L, Shahmoradi A, Brzózka MM, Wichert SP, Stadler AK, Nave KA, Rossner MJ Dynamic regulation of myelin genes in wild type and SHARP1 and -2 double-null mutant mice. In preparation.

Shahmoradi A, Brzózka MM, Reinecke L, Wichert SP, Oster H, McMahon FJ, Wehr MC, Schulze TG, Taneja R, Schmitt A, Falkai P, Paul C. Baier PC, Rossner MJ Sharp1 and -2 deficient mice display a spectrum of sleep-wake behavior associated endophenotypes of psychiatric diseases. In submission.

Wilhelm F, Winkler U, Morawski M, Jäger C, Reinecke L, Rossner MJ, Hirrlinger PG, Hirrlinger J The human ubiquitin C promoter drives selective expression in principal neurons in the brain of a transgenic mouse line. J.Neurochem Int. 2011 Nov;59(6):976-80. Epub 2011 Jul 23.

Rossner MJ, Oster H, Wichert SP, Reinecke L, Wehr MC, Reinecke J, Eichele G, Taneja R, Nave KA Disturbed clockwork resetting in Sharp-1 and Sharp-2 single and double mutant mice. PLoS One. 2008 Jul 23;3(7):e2762.

Wehr MC, Reinecke L, Botvinnik A, Rossner MJ Analysis of transient phosphorylationdependent protein-protein interactions in living mammalian cells using split-TEV. BMC Biotechnol. 2008 Jul 13;8:55. 